\title{
First Search at CDF for the Higgs Boson \\ Decaying to a $W$-Boson Pair \\ in Proton-Antiproton Collisions \\ at the Center-of-Mass Energy of 1.96 TeV
}

by

Shan-Huei S. Chuang

A dissertation submitted in partial fulfillment of the requirements for the degree of

Doctor of PhiLOSOPhY

(PhYsics)

at the

UNIVERSITY OF WISCONSIN - MADISON

2006 
(C) Copyright by Shan-Huei S. Chuang 2006

All Rights Reserved 


\section{Abstract}

By way of retaining the gauge invariance of the Standard Model (SM) and giving masses to the $W^{ \pm}$and $Z^{0}$ bosons and the fermions, the Higgs mechanism predicts the existence of a neutral scalar bosonic particle, whose mass is not exactly known. The Higgs boson is the only experimentally unconfirmed SM particle to date.

This thesis documents a search for the Higgs boson in $p \bar{p}$ collisions at $\sqrt{s}=$ $1.96 \mathrm{TeV}$ at the Tevatron, using $360 \pm 22 \mathrm{pb}^{-1}$ data collected by the Run II Collider Detector at Fermilab (CDF II), as part of the most important quest for contemporary particle physicists.

The search was for a Higgs boson decaying to a pair of $W^{ \pm}$bosons, where each $W$ boson decays to an electron, a muon or a tau that further decays to an electron or a muon with associated neutrinos. Events with two charged leptons plus large missing energy were selected in data triggered on a high $p_{t}$ lepton and compared to the signal and backgrounds modeled using Monte Carlo and jet data.

No signal-like excess was observed in data. Therefore, upper limits on the $H W W$ production cross-section in the analyzed mass range were extracted using the binned likelihood maximum from distributions of dilepton azimuthal angle at 95\% Bayesian credibility level (CL), as shown in the table below. Results of this analysis have been published in Physical Review Letters 97081802 (2006).

\begin{tabular}{ccccccccccc}
\hline \hline$m_{H}\left(\mathrm{GeV} / c^{2}\right)$ & 110 & 120 & 130 & 140 & 150 & 160 & 170 & 180 & 190 & 200 \\
\hline \hline expected $(\mathrm{pb})$ & 8.9 & 6.9 & 5.7 & 4.9 & 4.3 & 3.4 & 3.2 & 3.5 & 3.8 & 4.0 \\
\hline observed $(\mathrm{pb})$ & 8.3 & 4.5 & 4.4 & 4.6 & 3.5 & 3.2 & 3.4 & 4.3 & 5.5 & 5.2 \\
\hline \hline
\end{tabular}

Table 0.0: Summary of the expected and observed $\sigma(p \bar{p} \rightarrow H) \times \mathrm{BR}\left(H \rightarrow W^{+} W^{-}\right)$ production cross-section upper limits at $95 \% \mathrm{CL}$ as a function of the Higgs mass. 


\section{Acknowledgements}

First of all ${ }^{1}$, I thank my advisør and other members of the UW-CDF group for sending me to Fermilab. I thank Matt Herndon for supplying me knowledge of $b$ physics which I had lacked for long; I wished that he came to us a couple of years earlier.

I thank Wesley Smith for giving me a chance to work on ep collider physics at DESY before I jumped into $p \bar{p}$ collisions at the Tevatron. I thank Patrick Ryan and other classmates for being nuts and having fun with me before we moved on to different experiments. Thanks to Tao Han and other professors in the UW physics department for giving me and my classmates well organized and informative lectures.

I thank Pasha Murat, Eva Halkiadakis and Greg Veramendi for leading me into the real and really wonderful world of high energy physics (HEP) at CDF by way of studying the $E / p$ tail of electrons for the simulation of detector material amounts. It was great fun and I learned many HEP tools that have been useful in my research life ever since.

The biggest thanks I give to Mark Kruse, who, with extra care and excellent communication and research skills, brought me through several diboson physics analyses that went from $W^{+} W^{-}$cross-section measurement to the Higgs boson search in the $H \rightarrow W^{+} W^{-}$dilepton decay channels. In fact, what I followed was the best path I could think of; therefore I think I was the luckiest graduate student at CDF. Thank you, Mark.

\footnotetext{
${ }^{1}$ This work is sponsored by the Department of Energy of the United States with grant under contract DE-FG02-95ER40896.
} 
Another big thanks to Mark's postdocs, Susana Cabrera and Mircea Coca, who worked with me carefully and patiently through each aspect of the analyses and taught me details of experimental physics and a life philosophy for a young physicist. My life would not have been easy without you; my researches have been fruitful with your company. Thank you, Susana, Mircea and the rest of the Duke group for your tremendous help. I thank the godparents of the $H W W$ search, Bob Wagner, Andy Hocker and Daniel Whiteson, for their patience and careful review of the publication drafts. I also thank the CDF exotic physics group conveners, Beate Heineman and Song-Ming Wang, and the spokespersons, Rob Roser and Jaco Konigsberg, for their constant support and timely encouragement.

Thanks to Eiko, Carsten, Rio, Juan Pablo, Ernest... (throw me into the Fermi pool for not listing all the names) for being my friends and enjoying life with me through all those excursions and fun parties.

Much gratitude to Guillelmo Gomez-Ceballos for his immeasurable love, invaluable advices and encouraging words along the way of my PhD study. 


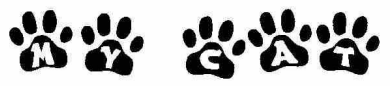

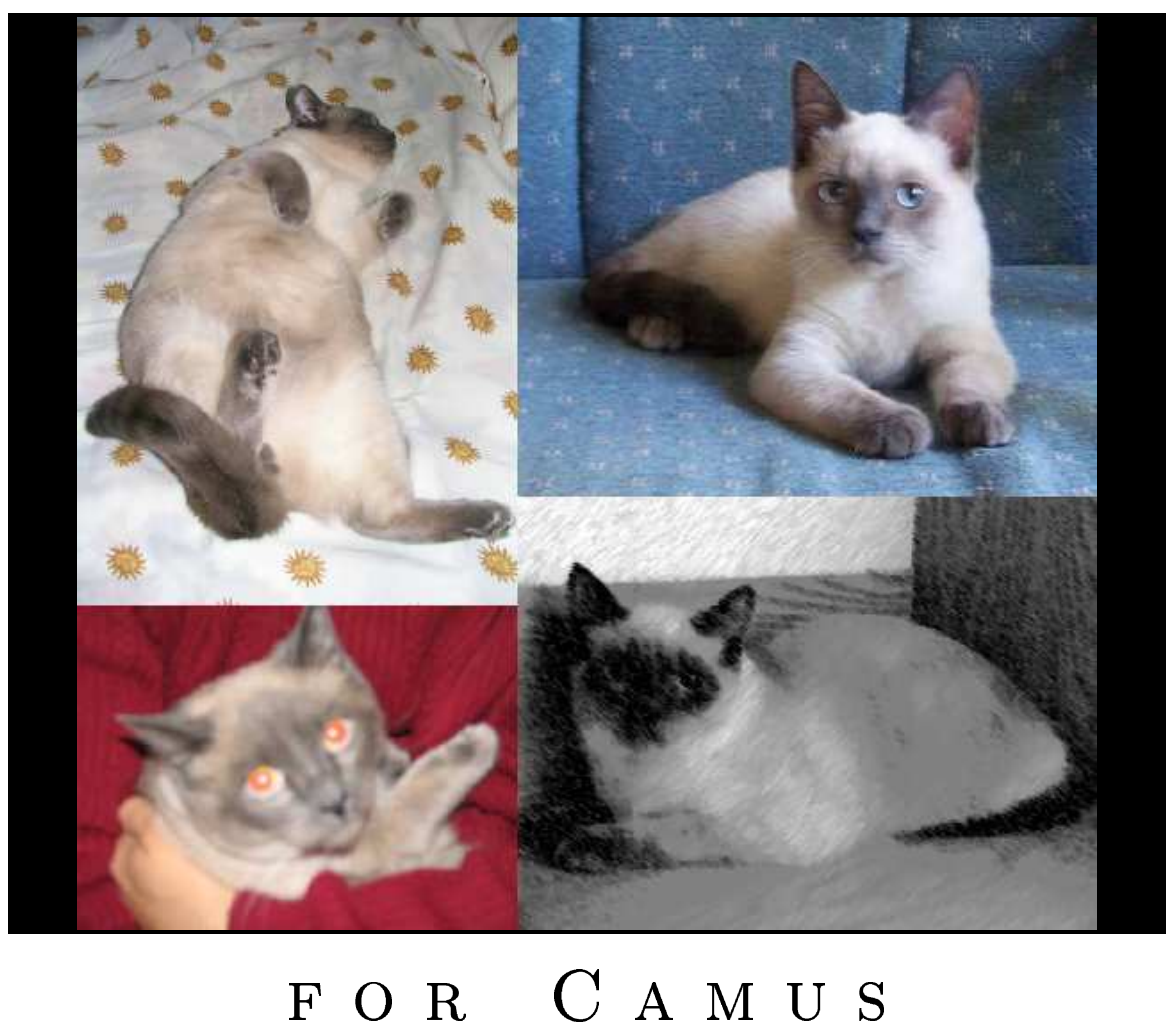

THE ABSOLUTELY BEAUTIFUL YET SUPERBLY SHY CAT 


\section{Contents}

$\begin{array}{ll}\text { Abstract } & \text { i }\end{array}$

$\begin{array}{ll}\text { Acknowledgements } & \text { iii }\end{array}$

1 Introduction $\quad 1$

1.1 The Standard Model . . . . . . . . . . . . . . . 1

1.1.1 Particles and Antiparticles .............. 2

1.1.2 Leptons .......................... 4

1.1.3 Quarks, Hadrons and Jets ............. 5

1.1.4 Gauge Bosons .................. 6

1.1.5 Symmetries and Groups .............. 8

1.1.6 Gauge Invariance . . . . . . . . . . . . . 10

1.1.7 Electroweak Unification . . . . . . . . . . . 10

1.1.7.1 Couplings ..................... 11

1.1.7.2 Masses ..................... 13

1.1.8 The Higgs Boson . . . . . . . . . . . . . . 18

1.1.9 Everything Together and Anything Else . . . . . . . . 18

1.2 Beyond the Standard Model . . . . . . . . . . . . . . 21 
2 Searches for the SM Higgs Boson $\quad 24$

2.1 Fits with Electroweak Data ............... 24

2.2 Direct Searches at LEP II . . . . . . . . . . . . . . . . . 27

2.3 Direct Searches at Tevatron . . . . . . . . . . . . . 30

2.3 .1 Run I .............................. 34

2.3 .2 Run II . . . . . . . . . . . . . . . . 36

3 Tevatron and the CDF II Detector $\quad 37$

3.1 Accelerator Complex at Fermilab . . . . . . . . . . . . 38

3.2 Overview of the Run II(a) Upgraded CDF Detector . . . . . . . . . 42

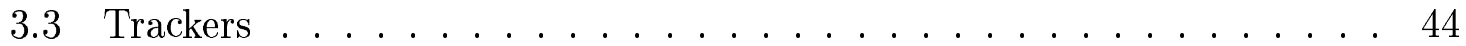

3.3.1 Superconducting Solenoid . . . . . . . . . . . . 45

3.3.2 Central Outer Tracker ............... . . 45

3.3.3 Silicon Vertex Detectors . . . . . . . . . . . 47

3.3.3.1 Run II(a) Upgraded Silicon Vertex Detector . . . . . . 48

3.3.3.2 Silicon Layer00 . . . . . . . . . . . . . 48

3.3.3.3 Intermediate Silicon Layers . . . . . . . . . . . . 49

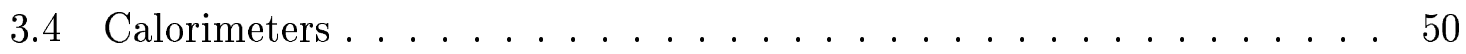

3.4.1 Central Calorimeters .................. 51

3.4.1.1 Central Electromagnetic Calorimeter . . . . . . . 51

3.4.1.2 Central Hadronic Calorimeter . . . . . . . . . 53

3.4.1.3 Wall Hadronic Calorimeter . . . . . . . . . 53

3.4.2 Endplug Calorimeters . . . . . . . . . . . . 54

3.4.2.1 Plug Electromagnetic Calorimeters ......... 54 
3.4.2.2 Plug Hadronic Calorimeters . . . . . . . . . 55

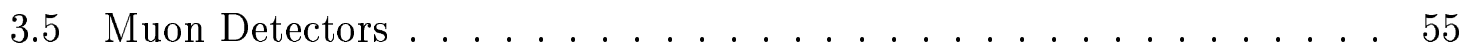

3.5.1 Central Muon Detector . . . . . . . . . . . 56

3.5.2 Central Muon uPgrade . . . . . . . . . . . 57

3.5.3 Central Muon eXtension ............... 61

3.5.4 Intermediate Muon Detector . . . . . . . . . . 61

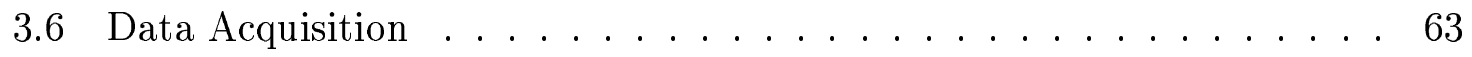

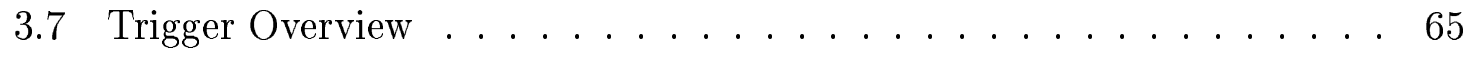

4 Data Description $\quad 67$

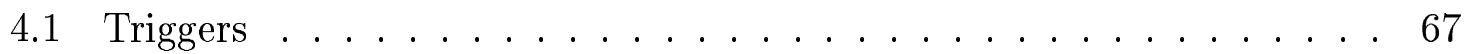

4.1.1 ELECTRON_CENTRAL_18 . . . . . . . . . . 67

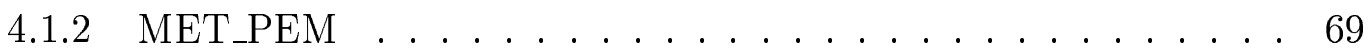

4.1 .3 MUON_CMUP18 ..................... 70

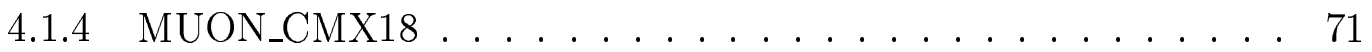

4.2 Good Run Lists . . . . . . . . . . . . . . . . 71

4.3 Integrated Luminosities $\ldots \ldots \ldots \ldots$. . . . . . . . . . . . . . . . .

4.3.1 Equivalent Integrated Luminosity . . . . . . . . . . . . 74

5 Particle Identification $\quad 75$

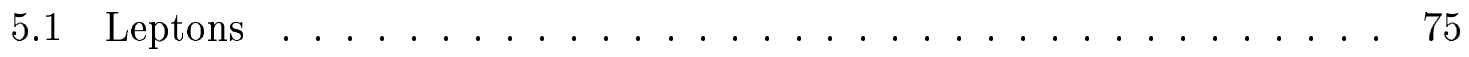

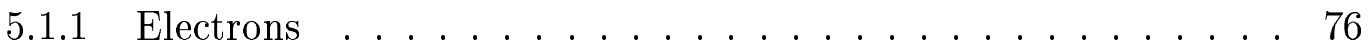

5.1.1.1 Tight Central Electrons ........... 76

5.1.1.2 Phoenix Electrons . . . . . . . . . . 79

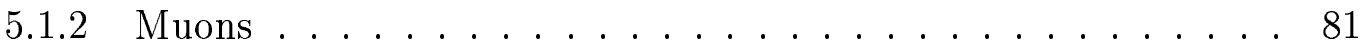


5.1.2.1 Minimum Ionizing Particles . . . . . . . . . . . 81

$5.1 .2 .2 \quad$ CMUP Muons . . . . . . . . . . . . 83

5.1.2.3 CMX Muons . . . . . . . . . . . . . . . . . 84

5.1.2.4 CMU-only Muons . . . . . . . . . . . . . 85

5.1.2.5 CMP-only Muons . . . . . . . . . . . . . . . . 85

5.1 .2 .6 Stubless Muons . . . . . . . . . . . . 86

5.1 .3 Trigger Leptons . . . . . . . . . . . . . . . . . 87

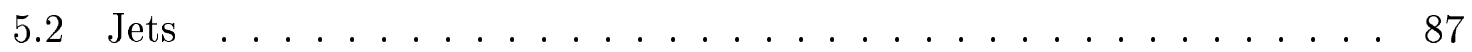

5.2 .1 Clustering Algorithm . . . . . . . . . . . . . . 89

5.2 .2 Energy Correction $\ldots \ldots \ldots \ldots \ldots$

5.2 .3 Jets for Veto Consideration . . . . . . . . . . . . 92

5.3 Missing Transverse Energy $\ldots \ldots \ldots$. . . . . . . . . . . 92

6 Estimate of Lepton Fake Rates $\quad 94$

$6.1 \mathrm{QCD} / W+$ jet Background Estimate Procedure $\ldots \ldots \ldots \ldots$

6.1.1 Lepton Fake Rate Estimate . . . . . . . . . . . . . . 95

6.1.1.1 Datasets . . . . . . . . . . . . . . 96

6.1.1.2 Fake Rate Definitions . . . . . . . . . . . 96

6.1.1.3 Lepton Selections . . . . . . . . . . . . . . . 97

6.1.1.4 Fakeable Object Selections . . . . . . . . . . . 97

6.1.1.5 Trigger Bias Reduction . . . . . . . . . . . . . 99

6.1.1.6 Results . . . . . . . . . . . . . 999

6.1.2 Lepton Fake Background Estimate . . . . . . . . . . . 112 
7 Event Selection $\quad 114$

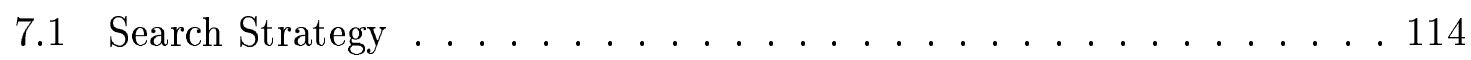

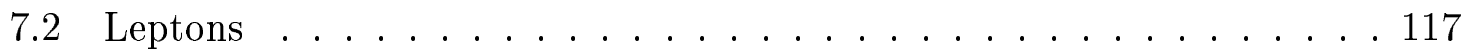

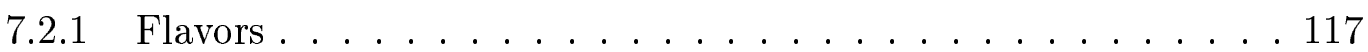

7.2.2 Transverse Energies . . . . . . . . . . . . 117

7.2.3 Charge Signs . . . . . . . . . . . . 120

7.3 Jets . . . . . . . . . . . . . . . . 121

7.4 Dilepton Invariant Mass . . . . . . . . . . . . . . . . . 124

7.5 Missing Transverse Energy . . . . . . . . . . . . . . 128

7.6 Azimuthal Angle between $Z_{t}$ and Other Objects . . . . . . . . . . . 130

7.7 Sum of Lepton and Missing Transverse Energies . . . . . . . . . . . 132

7.8 Summary of Event Selection Cuts . . . . . . . . . . . 133

8 Estimate of Signal Acceptance $\quad 136$

8.1 Signal Monte Carlo . . . . . . . . . . . . . . 136

8.2 Estimate of Acceptance . . . . . . . . . . . . . 137

8.2.1 Raw Acceptance ..................... 137

8.2.2 Correction for Trigger Efficiency . . . . . . . . . . 138

8.2.3 Correction for Lepton Reconstruction and Identification . . . . . 138

8.2.4 Correction for PHX Charge Misidentification . . . . . . . . 139

8.3 Estimate of Systematic Uncertainties . . . . . . . . . . 139

8.3.1 MC Generator and Parton Shower Model . . . . . . . . . 140

8.3.2 Initial State Radiation . . . . . . . . . . . . . . 140

8.3.3 Parton Distribution Function . . . . . . . . . . . 141 
8.3.4 The $\alpha_{s}$ Strong Coupling Strength . . . . . . . . . . . . . . 142

8.3.5 Trigger Efficiencies .................. 142

8.3.6 Lepton Reconstruction and Identification . . . . . . . . . . . 142

8.3.7 Track Isolation . . . . . . . . . . . . . . . 142

8.3.8 Jet Energy Scale . . . . . . . . . . . . . 144

8.3.9 Total Uncertainty ................... 145

8.4 Summary on Signal Acceptance . . . . . . . . . . . . . 145

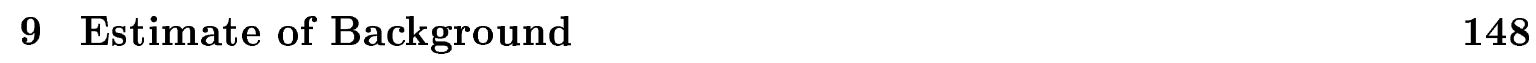

9.1 Background Sources . . . . . . . . . . . . . . 148

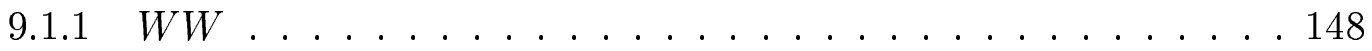

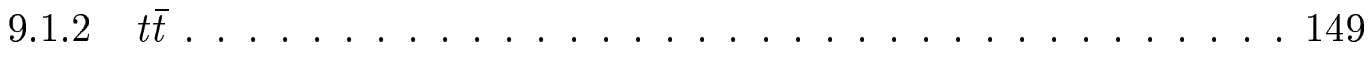

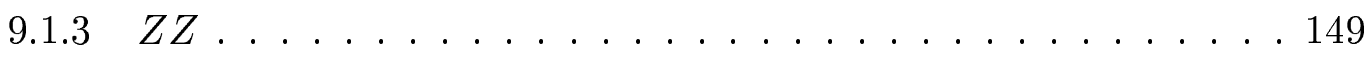

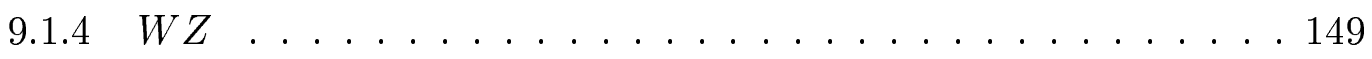

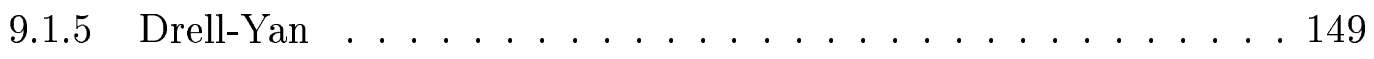

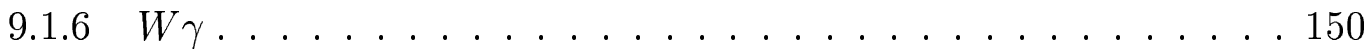

$9.1 .7 \mathrm{QCD} / W+$ jet $\ldots \ldots \ldots \ldots \ldots$

9.2 Estimate Procedure . . . . . . . . . . . . . . 152

9.2.1 Monte Carlo Approach . . . . . . . . . . . 152

9.2.2 Data Approach ..................... 155

9.3 Systematic Uncertainties in Background . . . . . . . . . . 155

9.4 Signal and Background Event Yields . . . . . . . . . 155

10 Limit Extraction $\quad 161$

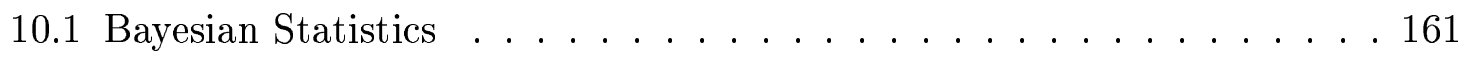


10.1.1 Bayes' Theorem .................... 163

10.1.2 Likelihood of Null Hypothesis . . . . . . . . . . . . . . 164

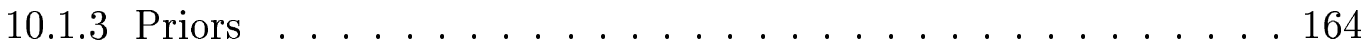

10.1.3.1 Signal Cross-Section . . . . . . . . . . . . 164

10.1.3.2 Signal Acceptance . . . . . . . . . . . 165

10.1.3.3 Integrated Luminosity . . . . . . . . . 166

10.1.3.4 Backgrounds . . . . . . . . . . . 167

10.1.4 Posterior . . . . . . . . . . . . . . 167

10.2 Binned Likelihood . . . . . . . . . . . . . . . 168

$10.395 \%$ CL Upper Limit . . . . . . . . . . . . . . 168

10.4 Numerical Computation . . . . . . . . . . . . 169

10.5 Pseudoexperiments . . . . . . . . . . . . . . 171

$10.695 \%$ CL Upper Limits on the $H W W$ Signal Cross-Section . . . . . . . 175 


\section{List of Tables}

1.1 Leptons in the Standard Model. . . . . . . . . . . . . . 4

1.2 Properties of leptons in the Standard Model. . . . . . . . . . . . 4

1.3 Quarks in the Standard Model. . . . . . . . . . . . . 5

1.4 Properties of quarks in the Standard Model. . . . . . . . . . 5

1.5 Gauge bosons in the Standard Model. . . . . . . . . . . . . . 7

2.1 Summary of the CDF Run I SM Higgs searches. . . . . . . . . . 35

3.1 Comparison of the silicon and COT tracking resolutions. . . . . . . 49

4.1 Run periods of TCE trigger dependence. . . . . . . . . . . 68

4.2 TCE trigger efficiencies. . . . . . . . . . . . . 68

4.3 TCE trigger efficiencies, requiring the silicon vertex detector in good

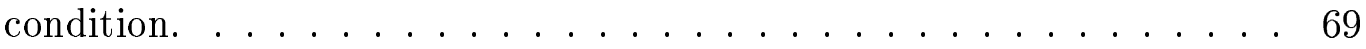

4.4 Good run lists and corresponding integrated luminosities. . . . . . . 72

5.1 PHX charge misidentification rates and scale factors. . . . . . . . 80

6.1 High $p_{t}$ lepton and jet datasets for lepton fake rate estimate. . . . . . 96

6.2 TCE selection cuts. . . . . . . . . . . . 100 
6.3 Fakeable electromagnetic object selection cuts. . . . . . . . 100

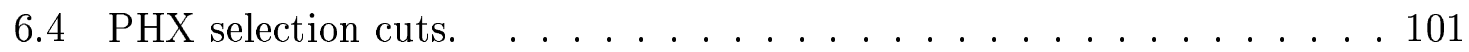

6.5 Muon selection cuts. . . . . . . . . . . . . 102

6.6 Fakeable MIP track selection cuts. . . . . . . . . . . . 103

6.7 Fake lepton predictions across jet samples (1). . . . . . . . . 109

6.8 Fake lepton predictions across jet samples (2). . . . . . . . 110

7.1 Signal MC event fraction as a function of jet multiplicity. . . . . . . 121

7.2 Pseudoexperimental limits as a function of $\not_{t}$ cut location. . . . . . 130

7.3 DY background reductions due to $\not_{t}>50 \mathrm{GeV}$ or $\Delta \phi_{\not_{t}, \ell / j}>20^{\circ}$. . 132

8.1 Information about the signal MC samples. . . . . . . . . . . 137

8.2 High $p_{t}$ lepton trigger efficiencies. . . . . . . . . . . . . 138

8.3 Lepton reconstruction and identification data/MC scale factors. . . . 139

8.4 PHX charge identification scale factors. . . . . . . . . 139

8.5 Information about the ISR-variant signal MC samples. . . . . . . . . 141

8.6 Track isolation fraction cut efficiencies and scale factors for reference. . 143

8.7 Summary of the systematic uncertainties in signal acceptance. . . . . . 145

8.8 Summary of the $H W W$ signal acceptance. . . . . . . . . . . . 146

9.1 Information about the background MC samples. . . . . . . . . . 154

9.2 Summary of the systematic uncertainties in backgrounds. . . . . . 156

9.3 Summary of the signal and background event yields for $m_{H}=110 \mathrm{GeV} .157$

9.4 Summary of the signal and background event yields for $m_{H}=120 \mathrm{GeV} .157$

9.5 Summary of the signal and background event yields for $m_{H}=130 \mathrm{GeV} .158$ 
9.6 Summary of the signal and background event yields for $m_{H}=140 \mathrm{GeV} .158$

9.7 Summary of the signal and background event yields for $m_{H}=150 \mathrm{GeV} .158$

9.8 Summary of the signal and background event yields for $m_{H}=160 \mathrm{GeV} .159$

9.9 Summary of the signal and background event yields for $m_{H}=170 \mathrm{GeV} .159$

9.10 Summary of the signal and background event yields for $m_{H}=180 \mathrm{GeV} .159$

9.11 Summary of the signal and background event yields for $m_{H}=190 \mathrm{GeV} .160$

9.12 Summary of the signal and background event yields for $m_{H}=200 \mathrm{GeV} .160$

10.1 Expected and observed $\sigma\left(p \bar{p} \rightarrow H \rightarrow W^{+} W^{-}\right)$upper limits. . . . . 174 


\section{List of Figures}

1.1 Dirac picture of pair production or annihilation. . . . . . . . . 3

1.2 Illustration of the Higgs scalar potential. . . . . . . . . . . . . 14

1.3 Feynman diagram for the Higgs boson coupling to fermions. . . . . . . 17

1.4 Energy scale of GUT. . . . . . . . . . . . . . . . . . 19

1.5 Constraints on the Higgs mass. . . . . . . . . . . . . . . . 20

1.6 Enhancement factors for the single Higgs production of the 4th Generation Model. ..................... 22

2.1 The latest $m_{W}$ and $m_{t}$ precision measurement results. . . . . . . 25

2.2 Contours of $m_{W}$ vs. $m_{t}$ on the $m_{H}$ grid. . . . . . . . . . . . . 26

2.3 Pull of the EWWG's global MSM fit. . . . . . . . . . . 26

$2.4 \Delta \chi^{2}$ of the EWWG's global MSM fit. . . . . . . . . . . . 27

2.5 Test statistic $-2 \ln Q$ of the LEP combined SM Higgs boson search. . . 28

2.6 Signal confidence $\mathrm{CL}_{s}$ of the LEP combined SM Higgs boson search. . 29

2.7 The SM Higgs production modes at the Tevatron. . . . . . . . . 31

2.8 The SM Higgs decay modes and branching fractions. . . . . . . . . . 31

2.9 Feynman diagram for $g g \rightarrow h^{0} \rightarrow W^{+} W^{-} \rightarrow \ell^{+} \nu_{\ell} \ell^{-} \bar{\nu}_{\ell} \ldots \ldots . . . . .32$

2.10 Potentials of direct SM Higgs searches at the Tevatron. . . . . . . . 34 
2.11 Results of the CDF Run I SM Higgs searches. . . . . . . . . . . 35

3.1 Aerial photograph of Fermilab. . . . . . . . . . . . . 38

3.2 Illustration of the Fermilab accelerator complex. . . . . . . . . . 39

3.3 History of the Tevatron's luminosity delivery. . . . . . . . . . . . 41

3.4 Longitudinal view of half of the CDF Run II(a) detector. . . . . . . 43

3.5 Longitudinal view of a quarter of the CDF II tracking system. . . . . . 44

3.6 Nominal layout of three COT cells. . . . . . . . . . . . 46

3.7 Photograph of the COT, taken in LBL. . . . . . . . . . 46

3.8 Schematic views of the silicon vertex detectors. . . . . . . . . . 47

3.9 Schematic view of a CEM wedge. . . . . . . . . . . . 52

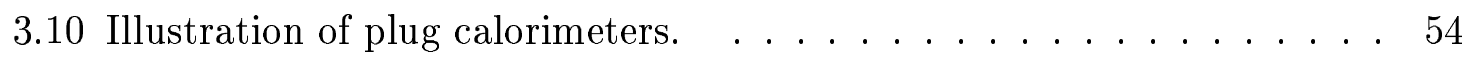

3.11 Transverse view of a CMU drift tube (left) and of a CMU stack (right). 56

3.12 Transverse view of the CMP geometry. . . . . . . . . . . 58

$3.13 \mathrm{CMP} / \mathrm{CMX} / \mathrm{IMU}$ drift tube design. $\ldots \ldots \ldots \ldots \ldots$

3.14 Transverse view of the CMX and CSX stacks. . . . . . . . . 60

3.15 Illustration of two jumpered BMU drift tubes. . . . . . . . . . . . 62

3.16 CDF II muon reconstruction and identification efficiencies. . . . . . . 63

3.17 Block diagram of the deadtimeless CDF II DAQ system. . . . . . . . 64

3.18 Block diagram of the CDF II trigger system. . . . . . . . . . 66

5.1 Lepton track isolation fraction distributions. $\ldots \ldots \ldots \ldots$

5.2 Jet fragmentation levels for the CDF II jet energy correction. $\quad \ldots \ldots$. . 91

$6.1 E_{t}$ distributions of jets in the high $p_{t}$ lepton and jet samples. $\ldots \ldots 99$ 
6.2 $E_{\text {had }} / E_{\text {em }}$ distributions of electromagnetic objects in the high $p_{t}$ lepton and jet samples. . . . . . . . . . . . . . . 101

$6.3 E_{\text {em }+ \text { had }} / p$ distributions of MIP tracks in the jet samples. . . . . . . 103

6.4 Cal isoFrac profiles vs. $E_{t}$ of fakeable objects in the jet samples. . . . 104

6.5 Cal isoFrac profiles vs. $E_{t}$ of fakeable object with cal isoFrac $<0.2$. . 105

$6.6 E_{t}$ distributions of leptons in the jet samples. . . . . . . . 106

6.7 $E_{t}$ distributions of fakeable objects in the high $p_{t}$ lepton and jet samples. 107

6.8 Lepton fake rates. . . . . . . . . . . . . 108

6.9 Lepton fake $\Delta \phi_{\ell \ell}$ distributions. . . . . . . . . . . . . . . 111

7.1 Framework for the $H W W$ dilepton angular distributions. . . . . . . 115

7.2 PYTHIA HEPG $H W W$ lepton $E_{t}$ distributions. . . . . . . . . . 118

7.3 Raw signal acceptances as a function of lepton $E_{t}$ thresholds. . . . . . 119

7.4 Reconstructed lepton $E_{t}$ distributions. . . . . . . . . . . 120

7.5 Jet multiplicity distributions. . . . . . . . . . . . 121

7.6 Jet $E_{t}$ distributions. . . . . . . . . . . . . . . . 122

$7.7 \mathrm{~S} / \sqrt{\mathrm{B}}$ as a function of jet multiplicity and jet $E_{t}$ veto thresholds. . . . 123

7.8 Dilepton invariant mass $m_{\ell \ell}$ distributions. . . . . . . . . . 125

$7.9 \mathrm{~S} / \sqrt{\mathrm{B}}$ as a function of $m_{\ell \ell}$ upper cut location. . . . . . . . 126

$7.10 \mathrm{~S} / \sqrt{\mathrm{B}}$-maximal $m_{\ell \ell}$ cut location as a function of the Higgs mass. . . . 127

7.11 Missing transverse energy $\not_{t}$ distributions. . . . . . . . . 128

$7.12 \mathrm{~S} / \sqrt{\mathrm{B}}$ as a function of $\not_{t}$ cut location. . . . . . . . . . . 129

$7.13 \#_{t}$ vs. $\Delta \phi_{E_{t}, \ell / j}$ distributions. . . . . . . . . . . 131

7.14 Sum of lepton and missing transverse energy distributions. . . . . . 133 
7.15 Sum of lepton and missing transverse energy distributions (N-1). . . 134

8.1 Electron and muon track isolation fraction distributions. . . . . . . 143

8.2 Summary of the $H W W$ signal acceptance. . . . . . . . . . . 146

9.1 Same-sign dilepton $\Sigma E_{t}^{\ell}+\not E_{t}$ distributions. . . . . . . . . 151

10.1 Dilepton azimuthal separation angle $\Delta \phi_{\ell \ell}$ distributions. . . . . . . 162

10.2 Observed $\sigma\left(p \bar{p} \rightarrow H \rightarrow W^{+} W^{-}\right)$likelihood distributions. . . . . . . 172

10.3 Expected $\sigma\left(p \bar{p} \rightarrow H \rightarrow W^{+} W^{-}\right)$upper limit distributions. . . . . . 173

10.4 Expected and observed $\sigma\left(p \bar{p} \rightarrow H \rightarrow W^{+} W^{-}\right)$upper limits. . . . . 174 


\section{Chapter 1}

\section{Introduction}

Particle physics is the study of fundamental constituents of matter and interactions between them. The study of particle physics began rather early in history; however, studies have moved deeper and deeper into matter as time goes by and knowledge improves - from earth, air, water and fire in ancient Chinese or Greek philosophies to the Standard Model of particle physics [1] in the modern world.

\subsection{The Standard Model}

The Standard Model (SM) [2, 3] attempts to explain the world at the subatomic level with elementary particles ${ }^{1}$ of three distinctive types: leptons, quarks and gauge bosons, where the bosons act as fundamental force carriers in particle interactions, following the guidance of symmetries and conservation laws and preserving the grace of transformation invariance. The Standard Model has experienced extraordinary success in its attempt; it explains all the observed phenomena of particle physics so

\footnotetext{
${ }^{1}$ By definition, an elementary particle has no internal structure, like a point, though it has mass, spin, momentum, energy and other properties of a particle.
} 
far.

\subsubsection{Particles and Antiparticles}

For every particle there is an antiparticle with equal mass but opposite charge, which is a necessary consequence of combining special relativity with quantum mechanics.

Suppose a free particle with rest mass $m$, momentum $\vec{p}$ and energy $E$ is described by de Broglie's wave function of space $\vec{x}$ and time $t$,

$$
\Psi(\vec{x}, t)=N \exp \left[\frac{i}{\hbar}(\vec{p} \cdot \vec{x}-E t)\right]
$$

where $\hbar$ is the plank constant $h$ divided by the speed of light $c, \hbar=\frac{h}{c}$, and $N$ is a normalization factor [4].

Non-relativistically, $E=\frac{p^{2}}{2 m}$ and the particle's motion is described by the Schrödinger equation [5]

$$
i \hbar \frac{\partial \Psi}{\partial t}(\vec{x}, t)=-\frac{\hbar^{2}}{2 m} \nabla^{2} \Psi(\vec{x}, t) .
$$

Relativistically, $E= \pm \sqrt{(p c)^{2}+\left(m c^{2}\right)^{2}}$ and the particle's motion is described by the Klein-Gordon equation [6]

$$
-\hbar^{2} \frac{\partial^{2} \Psi}{\partial t^{2}}(\vec{x}, t)=-(\hbar c)^{2} \nabla^{2} \Psi(\vec{x}, t)+\left(m c^{2}\right)^{2} \Psi(\vec{x}, t)
$$

or by the Dirac equation [7]

$$
i \hbar \frac{\partial \Psi}{\partial t}(\vec{x}, t)=H(\vec{x}, \hat{p}) \Psi(\vec{x}, t)
$$

where $\hat{p}=-i \hbar \nabla$ is a momentum operator; the Hamiltonian $H=\vec{\alpha} \cdot \hat{p} c+\beta m c^{2}$ where

$$
\vec{\alpha}=\left(\begin{array}{cc}
0 & \vec{\sigma} \\
\vec{\sigma} & 0
\end{array}\right) \quad \text { and } \quad \beta=\left(\begin{array}{cc}
I & 0 \\
0 & -I
\end{array}\right)
$$


and the Pauli matrices $\vec{\sigma}$ are defined in Equation (1.14). $\Psi(\vec{x}, t)$ in Equation (1.4) is a Dirac spinor, consisting of two sets (one for positive and the other for negative energy) of the two eigenstates $(|\uparrow\rangle$ and $|\downarrow\rangle$, conventionally called up and down) of a spin- $\frac{1}{2}$ particle. Dirac postulated [8] that all negative energy states are occupied and all positive energy states are unoccupied in a vacuum. Removing from a vacuum a particle with negative energy and a certain charge, leaving a "hole" in the vacuum, results in the observable phenomenon of a particle with positive energy and opposite charge; adding to the vacuum a particle with positive energy and opposite charge, the antiparticle of the removed particle by definition, results in the same observable phenomenon.

Take as an example pair production of electron $e^{-}$and its antiparticle, positron $e^{+}$, due to photon $\gamma$ energization - the movement of an electron from a negative energy state to a positive energy state results in the observable phenomenon of an electron with positive energy and a positron with positive energy; the process of $\gamma \rightarrow e^{+} e^{-}$is illustrated in Figure 1.1.

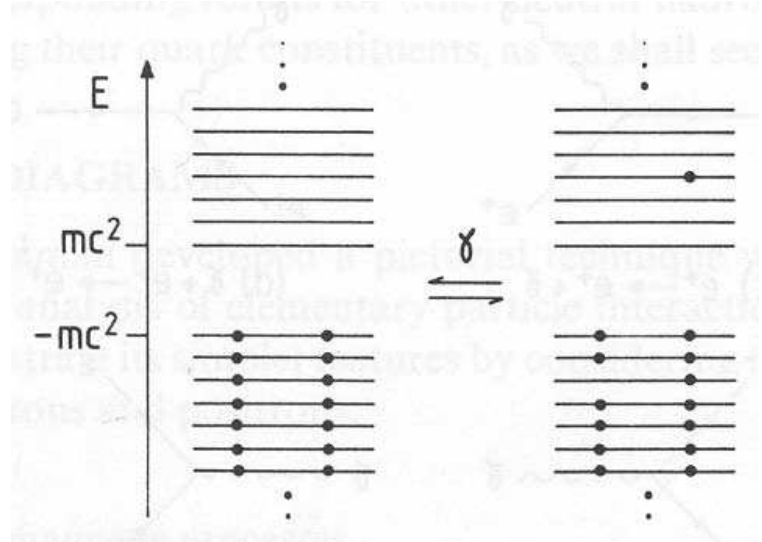

Figure 1.1: Dirac picture of particle pair production or annihilation via photon. 
The Dirac equation and the hole theory apply only to fermions, defined as particles with integer-and-half spin. Relativistic quantum field theory provides a generalized interpretation of antimatter, which apply to bosons as well as fermions. Antimatter appears as matter moving backwards in time.

\subsubsection{Leptons}

Leptons are fermions with spin $\frac{1}{2}$. There are three generations of leptons, as listed in Table 1.1, including electron $e^{-}$, muon $\mu^{-}$, tau lepton $\tau^{-}$and each one's associated antilepton and neutrinos.

The charged leptons interact electromagnetically as mediated by the photon and

\begin{tabular}{|l|l|l||l|l|l|}
\hline \hline \multicolumn{2}{|l||l||l|}{ antileptons } \\
\hline \hline$e^{-}$ & $\mu^{-}$ & $\tau^{-}$ & $\bar{\nu}_{e}$ & $\bar{\nu}_{\mu}$ & $\bar{\nu}_{\tau}$ \\
$\nu_{e}$ & $\nu_{\mu}$ & $\nu_{\tau}$ & $e^{+}$ & $\mu^{+}$ & $\tau^{+}$ \\
\hline \hline
\end{tabular}

Table 1.1: Leptons in the Standard Model.

\begin{tabular}{lcllc}
\hline \hline $\begin{array}{c}\text { sym } \\
\text {-bol }\end{array}$ & $\begin{array}{c}\text { mass } \\
\left(\mathrm{MeV} / c^{2}\right)\end{array}$ & $\begin{array}{c}\text { lifetime } \\
(\mu \mathrm{s})\end{array}$ & $\begin{array}{c}\text { decay } \\
\text { mode }\end{array}$ & $\begin{array}{c}\text { branching } \\
\text { fraction }(\%)\end{array}$ \\
\hline \hline$\nu_{e}$ & $<0.000002$ & stable & & \\
\hline$\nu_{\mu}$ & $<0.19$ & stable & & \\
\hline$\nu_{\tau}$ & $<18.2$ & stable & & \\
\hline$e^{ \pm}$ & 0.511 & stable & & 100.0 \\
\hline$\mu^{ \pm}$ & 105.658 & 2.197 & $e^{+} \nu_{e} \bar{\nu}_{\mu}$ & $17.36(5)$ \\
\hline$\tau^{ \pm}$ & $1777.0(3)$ & $2.906(10) \times 10^{-7}$ & $\mu^{+} \nu_{\mu} \bar{\nu}_{\tau}$ & $17.84(5)$ \\
& & $e^{+} \nu_{e} \bar{\nu}_{\tau}$ & \\
& & hadrons & $\sim 64.8$ \\
\hline \hline
\end{tabular}

Table 1.2: Masses, lifetimes, decay modes and branching fractions of leptons and an-

tileptons in the Standard Model [9]; the limits are at 95\% CL except on the muon neutrino mass, which is at $90 \%$ CL. 
weakly as mediated by $W^{ \pm}$and $Z^{0}$ gauge bosons, while the neutrinos only interact weakly. Lepton quantum numbers, defined as

$$
L_{\ell} \equiv N\left(\ell^{-}\right)-N\left(\ell^{+}\right)+N\left(\nu_{\ell}\right)-N\left(\bar{\nu}_{\ell}\right)
$$

where $\ell \in\{e, \mu, \tau\}$, and electric charge are always conserved in leptonic interactions. An example of a leptonic weak interaction is the decay of $W^{-} \rightarrow \ell^{-} \bar{\nu}_{\ell}$, in which lepton number $L_{\ell}=0$ and charge $Q=-1$ are conserved.

Basic lepton properties, such as mass, lifetime, decay mode and branching fraction (BR), are listed in Table 1.2.

\subsubsection{Quarks, Hadrons and Jets}

Quarks are fermions with spin $\frac{1}{2}$, electric charge $\pm \frac{1}{3}$ or $\pm \frac{2}{3}$, and an extra degree of freedom that is conventionally called color. Quarks $q$ and antiquarks $\bar{q}$, as listed in Table 1.3, are spinor fields in three flavors, each in the fundamental triplet repre-

\begin{tabular}{|l|l||l|l||l|l|}
\hline \hline \multicolumn{2}{|c|}{ (anti)quarks: symbol $\mid$ name } \\
\hline \hline$(\bar{u}) u$ & up & $(\bar{c}) c$ & charm & $(\bar{t}) t$ & top \\
$(\bar{d}) d$ & down & $(\bar{s}) s$ & strange & $(\bar{b}) b$ & bottom \\
\hline \hline
\end{tabular}

Table 1.3: Quarks in the Standard Model.

\begin{tabular}{|c|c|c|c|c|c|c|c|c|}
\hline \multirow{2}{*}{$\begin{array}{l}\text { sym } \\
\text {-bol }\end{array}$} & \multirow{2}{*}{$\begin{array}{c}\text { mass } \\
\left(\mathrm{GeV} / c^{2}\right)\end{array}$} & \multirow{2}{*}{$\begin{array}{c}\text { charge } \\
(e)\end{array}$} & \multicolumn{6}{|c|}{ quantum numbers } \\
\hline & & & $\tilde{s}$ & $\tilde{c}$ & $\tilde{b}$ & $\tilde{t}$ & $B$ & $I$ \\
\hline$\overline{d e}$ & $(1.5-3) \times 10^{-3}$ & $-\frac{1}{3}$ & 0 & 0 & 0 & 0 & & $\frac{1}{2}$ \\
\hline$u$ & $(3-7) \times 10^{-3}$ & $+\frac{2}{3}$ & 0 & 0 & 0 & 0 & $\frac{1}{3}$ & $\frac{1}{2}$ \\
\hline$s$ & $0.095 \pm 0.025$ & $-\frac{1}{3}$ & -1 & 0 & 0 & 0 & $\frac{3}{3}$ & 0 \\
\hline$c$ & $1.25 \pm 0.09$ & $+\frac{2}{3}$ & 0 & 1 & 0 & 0 & $\frac{0}{3}$ & 0 \\
\hline$b$ & $4.20 \pm 0.07$ & $-\frac{1}{3}$ & 0 & 0 & -1 & 0 & $\frac{1}{3}$ & 0 \\
\hline$t$ & $171.4 \pm 2.1$ & $+\frac{2}{3}$ & 0 & 0 & 0 & 1 & $\frac{1}{3}$ & 0 \\
\hline
\end{tabular}

Table 1.4: Masses, charges and quantum numbers of quarks in the Standard Model [9]. 
sentation of the color gauge group, $\mathrm{SU}(3)$. Gluons $g$ are vector fields in the adjoint octet representation of color $\mathrm{SU}(3)$. Quarks are bound in color singlet states, called hadrons, that have integer electric charge and zero color charge. The $q \bar{q}$ bound states are called mesons and the $q q q$ bound states are called baryons while other possibilities are either exotic or forbidden.

Quarks interact strongly, as mediated by the gluon; quarks also interact electromagnetically and weakly. Hadrons typically have lifetimes $10^{-22}-10^{-24} \mathrm{~s}$ in strong decays, $10^{-16}-10^{-21} \mathrm{~s}$ in electromagnetic decays and $10^{-7}-10^{-13} \mathrm{~s}$ in weak decays. Quantum numbers $\tilde{q} \equiv \pm[N(q)-N(\bar{q})]$ are conserved in strong and electromagnetic but not weak interactions. Baryon number $B=\frac{1}{3}[N(\Sigma q)-N(\Sigma \bar{q})]$ and electric charge are conserved in all interactions. Basic quark quantum numbers as well as mass and charge are listed in Table 1.4 .

Quarks and gluons, summarily called partons, fragment into jets of hadrons due to color confinement and strong interactivity.

The theory that describes strong interactions is called quantum chromodynamics (QCD). The theory that describes electromagnetic interactions is called quantum electrodynamics (QED). Although not as extensively tested as QED, QCD is nevertheless in good agreement with the bulk of experimental data to date.

\subsubsection{Gauge Bosons}

Gauge bosons are force carriers ${ }^{2}$, among which the photon carries the electromag-

\footnotetext{
${ }^{2}$ Graviton is spin-2, massless and electrically neutral; it carries the gravitational force in an infinitely long range with strength $10^{-39}$ relative to the strong coupling in the case of a proton; the gravitational force is generally negligible in comparison to the forces listed in Table 1.5.
} 


\begin{tabular}{|c|c|c|c|c|c|c|}
\hline sym & mass & force & coupling & range & decay & branching \\
\hline -bol & $\left(\mathrm{GeV} / c^{2}\right)$ & \multirow{2}{*}{$\begin{array}{l}\text { electro- } \\
\text { magnetic }\end{array}$} & strength & $(\mathrm{fm})$ & mode & fraction (\%) \\
\hline$\gamma$ & 0 & & $11^{-2}$ & $\infty$ & & \\
\hline$g$ & 0 & strong & 1 & 1 & & \\
\hline \multirow[t]{4}{*}{$W^{ \pm}$} & $80.403(29)$ & weak & $10^{-6}$ & $10^{-3}$ & $e \nu_{e}$ & $10.75(13)$ \\
\hline & & & & & $\mu \nu_{\mu}$ & $10.57(15)$ \\
\hline & & & & & $\tau \nu_{\tau}$ & $11.25(20)$ \\
\hline & & & & & hadrons & $67.60(27)$ \\
\hline \multirow[t]{3}{*}{$Z^{0}$} & $91.1876(21)$ & weak & $10^{-6}$ & $10^{-3}$ & $\ell^{+} \ell^{-}$ & $10.0974(69)$ \\
\hline & & & & & $\nu \bar{\nu}$ & $20.00(6)$ \\
\hline & & & & & hadrons & 69.91(6) \\
\hline
\end{tabular}

Table 1.5: Masses, force strengths and ranges, decay modes and branching fractions of spin-1 gauge bosons in the Standard Model [9].

netic force and the gluon carries the strong force while the $W^{ \pm}$and $Z^{0}$ bosons each carry a weak force. Basic properties of the gauge bosons with spin 1 are listed in Table 1.5. The $W^{ \pm}$and $Z^{0}$ bosons are massive and each decays approximately $30 \%$ to leptons and $70 \%$ to hadrons.

The simplest way of describing weak interactions of quarks combines two concepts: lepton-quark symmetry ${ }^{3}$

$$
\left(\begin{array}{c}
\nu_{e} \\
e
\end{array}\right)\left(\begin{array}{c}
\nu_{\mu} \\
\mu
\end{array}\right)\left(\begin{array}{c}
\nu_{\tau} \\
\tau
\end{array}\right) \Longleftrightarrow\left(\begin{array}{c}
u \\
d^{\prime}
\end{array}\right)\left(\begin{array}{c}
c \\
s^{\prime}
\end{array}\right)\left(\begin{array}{c}
t \\
b^{\prime}
\end{array}\right)
$$

and mixing within quarks

$$
\left(\begin{array}{c}
d^{\prime} \\
s^{\prime} \\
b^{\prime}
\end{array}\right)=\left(\begin{array}{lll}
V_{u d} & V_{u s} & V_{u b} \\
V_{c d} & V_{c s} & V_{c b} \\
V_{t d} & V_{t s} & V_{t b}
\end{array}\right)\left(\begin{array}{c}
d \\
s \\
b
\end{array}\right)
$$

\footnotetext{
${ }^{3}$ Prediction of the top quark had been in fact inspired by the lepton-quark symmetry since 1975 and was followed by the discovery at CDF in 1995 .
} 
where the coupling strength modification constants,

$$
\left(\begin{array}{ccc}
V_{u d} & V_{u s} & V_{u b} \\
V_{c d} & V_{c s} & V_{c b} \\
V_{t d} & V_{t s} & V_{t b}
\end{array}\right) \approx\left(\begin{array}{ccc}
1 & \theta_{c} & 0 \\
-\theta_{c} & 1 & 0 \\
0 & 0 & 1
\end{array}\right)
$$

can be approximately expressed with the Cabibbo quark mixing angle $\theta_{C}=(13.0 \pm$ $0.3)^{\circ}$ in a Cabibbo-Kobayashi-Maskawa (CKM) matrix [10].

Charge conjugation $C$, parity $P$ and the two together $C P$ are not necessary conserved in weak interactions. Quark numbers are not necessarily conserved in charged weak currents.

\subsubsection{Symmetries and Groups}

The group theory [11] is a branch of math that underlies the treatment of symmetry.

Rotation in the space of three dimensions is an example of a symmetry group. Rotations of a system form a group with the following properties:

1. group elements are closed under multiplication - two successive rotations $R_{1} R_{2}$ are equivalent to another single rotation;

2. group elements are associative though may not be commutative $-R_{3}\left(R_{2} R_{1}\right)=$ $\left(R_{3} R_{2}\right) R_{1}$ although $R_{1} R_{2} \neq R_{2} R_{1}$

3. there is an identity element - no rotation $R^{0}=1$;

4. every element has an inverse - reverse rotation $R^{-1}$.

In fact, rotations form a continuous Lie group since a finite rotation can be expressed as combination of infinitesimal rotations. 
In quantum mechanics, the elements of a symmetry group are represented by unitary transformations of states. Suppose a system has states transforming by a rotation operator $U(R)$ as

$$
\begin{aligned}
|\psi\rangle \rightarrow\left|\psi^{\prime}\right\rangle & =U(R)|\psi\rangle \\
|\phi\rangle & \rightarrow\left|\phi^{\prime}\right\rangle=U(R)|\phi\rangle .
\end{aligned}
$$

The amplitude for the system described by state $|\psi\rangle$ to be found in state $|\phi\rangle$ is invariant,

$$
\begin{aligned}
\langle\phi \mid \psi\rangle \rightarrow\left\langle\phi^{\prime} \mid \psi^{\prime}\right\rangle & =\left\langle\phi\left|U(R)^{\dagger} U(R)\right| \psi\right\rangle \\
& =\langle\phi \mid \psi\rangle
\end{aligned}
$$

and is unchanged by $U(R)-U(R)$ must be unitary. Rotation operators form a unitary representation of the rotation group.

In the lowest-dimensional nontrivial representation of the rotation group, the rotation generators for angular momentum $j=\frac{1}{2}$ may be written as $J_{i}=\frac{1}{2} \sigma_{i}$, where

$$
\sigma_{1}=\left(\begin{array}{ll}
0 & 1 \\
1 & 0
\end{array}\right) \quad \sigma_{2}=\left(\begin{array}{rr}
0 & -i \\
i & 0
\end{array}\right) \quad \sigma_{3}=\left(\begin{array}{rr}
1 & 0 \\
0 & -1
\end{array}\right)
$$

are the Pauli matrices [12], and the eigenstates of $\sigma_{3}$

$$
\left(\begin{array}{l}
0 \\
1
\end{array}\right) \text { and }\left(\begin{array}{l}
1 \\
0
\end{array}\right)
$$

are often chosen to be the basis spinors.

The Pauli matrices are hermitian and traceless. The rotation matrices by an arbitrary angle $\theta, U\left(R_{i}\right)=e^{-\frac{i}{2} \theta_{i} \sigma_{i}}$, are unitary and preserve the $\operatorname{determinant} \operatorname{det}\left(e^{i \sigma}\right)=1$ in matrix multiplication. The set of all unitary $N \times N$ matrices form a $U(N)$ group 
and the set of traceless unitary $N \times N$ matrices form a subgroup $S U(N)$ of $U(N)$, where $N=1,2,3 \ldots$ for example, the three color charges of a quark form the fundamental representation of an $S U(3)$ group; the two eigenstates of a spin- $\frac{1}{2}$ particle form the fundamental representation of an $S U(2)$ group.

\subsubsection{Gauge Invariance}

Transformation of a potential field with scalar $\phi(\vec{x}, t)$ and vector $\vec{A}(\vec{x}, t)$ components,

$$
\begin{aligned}
& \phi \quad \rightarrow \quad \phi^{\prime}=\phi+\frac{\partial f}{\partial t} \\
& \vec{A} \rightarrow \overrightarrow{A^{\prime}}=\vec{A}-\nabla f
\end{aligned}
$$

where $f(\vec{x}, t)$ is an arbitrary scalar function, is called a local gauge transformation. A theory, of which the predictions are unaltered by such a transformation with a corresponding transformation of wave function,

$$
\Psi \rightarrow \Psi^{\prime}=e^{-i g f} \Psi(\vec{x}, t)
$$

where $g$ is a constant, is said to be gauge invariant $[13,14]$. Gauge invariance underlies QED, QCD, electroweak unification and any theory in which the force carriers are spin-1 gauge bosons.

\subsubsection{Electroweak Unification}

Electromagnetic and weak interactions are unified in a theory that relates various $\gamma, W^{ \pm}$and $Z^{0}$ couplings by requiring an exact cancellation of divergences from higher order terms in all processes, which necessitates the use of gauge principle [15]. 


\subsubsection{Couplings}

In the simplest way [16], the electroweak model can be illustrated by the interactions of the electron $e$ and its associated neutrino $\nu_{e}$ where it requires local gauge symmetry under the transformation via weak isospin operators

$$
I_{i}^{W} \equiv \frac{1}{2} \sigma_{i}
$$

With the wave function defined as

$$
\Psi \equiv\left(\begin{array}{c}
\nu_{e} \\
e
\end{array}\right)
$$

where $\nu_{e}$ and $e$ denote the left-handed components of the $\nu_{e}$ and $e$, gauge transformation of the electroweak potential field $(\omega, \vec{W})$ and of the wave function $\Psi$ can be written as

$$
\begin{aligned}
\omega_{i} \rightarrow \omega_{i}^{\prime} & =\omega_{i}+\frac{\partial f_{i}}{\partial t}+g \sum_{j k} \epsilon_{i j k} f_{j} \omega_{k} \\
\vec{W}_{i} \rightarrow \vec{W}_{i}^{\prime} & =\vec{W}_{i}-\nabla f_{i}+g \sum_{j k} \epsilon_{i j k} f_{j} \vec{W}_{k} \\
\Psi \rightarrow \Psi^{\prime} & =e^{-i g \vec{I}^{W} \cdot \vec{f}} \Psi(\vec{x}, t) \\
& =\exp \left[-i g \sum_{i} I_{i}^{W} f_{i}\right] \Psi(\vec{x}, t)
\end{aligned}
$$

where $\epsilon_{i j k}$ is the completely antisymmetric tensor with $\epsilon_{123,231,312}=1$. Neglecting the electron and neutrino masses and including the weak isospin interaction terms, the Dirac equation (1.4) in respect of gauge symmetry can be modified to

$$
i\left(\frac{\partial}{\partial t}+i g \sum_{i} I_{i}^{W} \omega_{i}\right) \Psi=-i \alpha \cdot\left(\nabla-i g \sum_{i} I_{i}^{W} \vec{W}_{i}\right) \Psi .
$$

The first row of Equation (1.25), which is for the neutrino, can be written as

$$
i\left(\frac{\partial}{\partial t}+\alpha \cdot \nabla\right) \nu_{e}=\underbrace{\frac{g}{2}\left(\omega_{3}-\alpha \cdot \vec{W}_{3}\right) \nu_{e}}_{\nu_{e} \rightarrow \nu_{e} W^{0}}+\underbrace{\frac{g}{\sqrt{2}} g_{W}\left(\omega^{+}-\alpha \cdot \vec{W}^{+}\right) e^{-}}_{\nu_{e} \rightarrow e^{-} W^{+}} .
$$


The second row of Equation (1.25), which is for the electron, can be written as

$$
i\left(\frac{\partial}{\partial t}+\alpha \cdot \nabla\right) e^{-}=\underbrace{-\frac{g}{2}\left(\omega_{3}-\alpha \cdot \vec{W}_{3}\right) e^{-}}_{e^{-} \rightarrow e^{-} W^{0}}+\underbrace{\frac{g}{\sqrt{2}} g_{W}\left(\omega^{-}-\alpha \cdot \vec{W}^{-}\right) \nu_{e}}_{e^{-} \rightarrow \nu_{e} W^{-}} .
$$

For both Equation (1.26) and Equation (1.27),

$$
\omega^{ \pm}=\frac{1}{\sqrt{2}}\left(\omega_{1} \pm \omega_{2}\right) \quad \text { and } \quad \vec{W}^{ \pm}=\frac{1}{\sqrt{2}}\left(\vec{W}_{1} \pm \vec{W}_{2}\right)
$$

Four weak potentials are indicated with the corresponding process in Equation (1.26)(1.27), among which the neutral weak $W^{0}$ processes are experimentally unconfirmed. However, with an additional set of neutral weak ( $B^{0}$ by convention) processes that are associated to $W^{0}$ by a weak hypercharge $Y^{W} \equiv Q-I_{3}^{W}$, the experimentally confirmed electromagnetic and weak neutral currents $\gamma$ and $Z^{0}$ can then be expressed by linear combinations of $W^{0}$ and $B^{0}$ as

$$
\begin{aligned}
\gamma & =B^{0} \cos \theta_{w}+W^{0} \sin \theta_{w} \\
Z^{0} & =-B^{0} \sin \theta_{w}+W^{0} \cos \theta_{w}
\end{aligned}
$$

where $\theta_{w}$ is the Weinberg weak mixing angle [17].

To satisfy QED constraints that the photon does not couple to neutrinos and the coupling of photon to electrons remains the same with and without electroweak unification, the following condition is made

$$
e=g_{W} \sin \theta_{w}=g_{Z} \cos \theta_{w}
$$

where the Weinberg angle is

$$
\sin ^{2} \theta_{w}=1-\left(\frac{m_{W}}{m_{Z}}\right)^{2} \approx 0.23108(5)
$$


and $g_{W}$ and $g_{Z}$ are coupling constants that characterize leptonic $W^{ \pm}$and $Z^{0}$ vertices respectively.

\subsubsection{Masses}

In QED and QCD the force mediators $\gamma$ and $g$ are massless. In the electroweak theory, the $W^{ \pm}$and $Z^{0}$ each have mass about $100 \mathrm{GeV} / c^{2}$; however, because addition of the $W^{ \pm}$and $Z^{0}$ mass terms would destroy gauge invariance, an additional scalar field

$$
\phi=\left(\begin{array}{c}
\phi_{a} \equiv \phi_{1, a}+i \phi_{2, a} \\
\phi_{b} \equiv \phi_{1, b}+i \phi_{2, b}
\end{array}\right)
$$

is introduced to give the $W^{ \pm}$and $Z^{0}$ boson masses, by which way the electroweak gauge symmetry is spontaneously broken.

Since mass is an energy term, the best way to approach the problem is Lagrangian $\mathfrak{L} \equiv$ (kinetic energy - potential energy) that describes a system of multiple variables $\phi\left[x_{\mu} \equiv(\vec{x}, t)\right]$ by the Euler-Lagrangian equation

$$
\frac{\partial}{\partial x_{\mu}}\left[\frac{\partial \mathfrak{L}}{\partial\left(\frac{\partial \phi}{\partial x_{\mu}}\right)}\right]-\frac{\partial \mathfrak{L}}{\partial \phi}=0
$$

For a gauge boson with mass $m$ described by field $\phi$, the free-field Lagrangian is

$$
\mathfrak{L}=\frac{1}{2}\left(\partial_{\mu} \phi\right)\left(\partial^{\mu} \phi\right)-\frac{1}{2} m^{2} \phi^{2}
$$

For a Dirac spinor,

$$
\mathfrak{L}=i \bar{\psi} \gamma_{\mu} \partial^{\mu} \psi-m \bar{\psi} \psi
$$

where $\gamma^{\mu} \equiv(\beta, \beta \bar{\alpha})$. In compliance with the gauge principle for QED, an electromag-

netic interaction term $A_{\mu}$ as well as the associated covariant derivative $D_{\mu}$ for the photon field transforming as

$$
A_{\mu} \rightarrow A_{\mu}^{\prime}=A_{\mu}-\frac{1}{e} \partial_{\mu} f
$$




$$
D_{\mu} \equiv \partial_{\mu}-i e A_{\mu}
$$

are introduced so that the Lagrangian of QED

$$
\mathfrak{L}=\bar{\psi}\left(i \gamma^{\mu} \partial_{\mu}\right) \psi+e \bar{\psi} \gamma^{\mu} A_{\mu} \psi-\frac{1}{4} F_{\mu \nu} F^{\mu \nu}
$$

is gauge invariant, where the term with $F_{\mu \nu}^{(\mu \nu)} \equiv \partial_{\mu}^{(\mu)} A_{\nu}^{(\nu)}-\partial_{\nu}^{(\nu)} A_{\mu}^{(\mu)}$ corresponds to the kinetic energy term in Equation (1.35); there is no corresponding term to the potential energy term in Equation (1.35) because $m_{\gamma}=0$. In fact, photon mass has to be zero; otherwise the gauge invariance of QED would break.

To keep gauge invariance of the electroweak theory, mass and self-coupling terms of the scalar field $\phi$ replaces the mass term in Equation (1.35) so that

$$
\mathfrak{L}=\left(\partial_{\mu} \phi\right)^{\dagger}\left(\partial^{\mu} \phi\right)-\mu^{2} \phi^{\dagger} \phi-\lambda\left(\phi^{\dagger} \phi\right)^{2}
$$

where $\lambda>0$ and $\mu^{2}<0$.

The scalar field potential is shown in Figure 1.2. The minimum of potential at

$$
\left|\phi_{\min }\right|=\sqrt{\frac{-\mu^{2}}{\lambda}}
$$

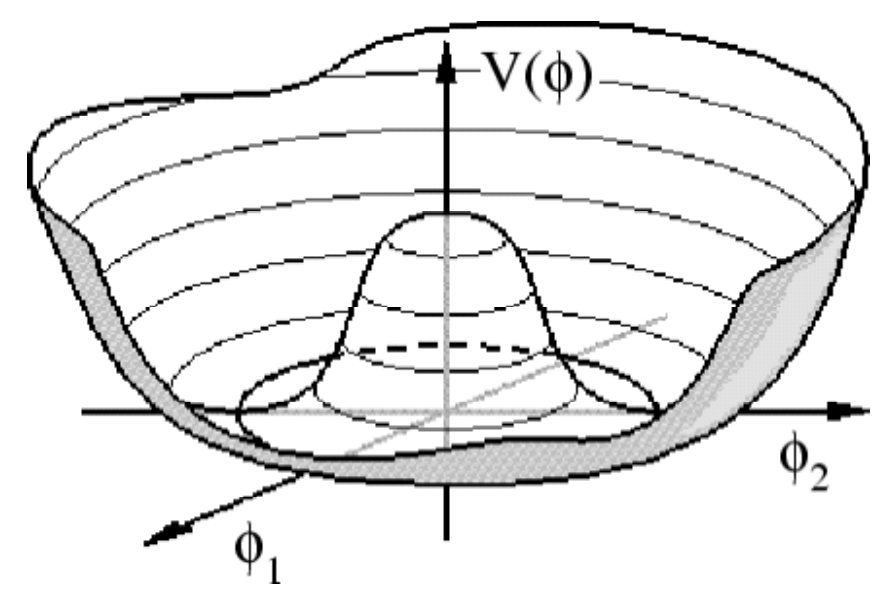

Figure 1.2: Illustration of the Higgs scalar potential. 


$$
\begin{aligned}
& =\frac{1}{\sqrt[4]{2} G_{F}} \\
& \approx 246 \mathrm{GeV}
\end{aligned}
$$

is presumed to be the vacuum expectation value (VEV), where the Fermi coupling constant $G_{F}=1.166 \times 10^{-5} \mathrm{GeV}^{-2}$.

An expansion around the non-zero potential minimum

$$
\begin{aligned}
\phi\left(x_{\mu}\right) & =\frac{1}{\sqrt{2}}\left[\phi_{\min }+h\left(x_{\mu}\right)+i G\left(x_{\mu}\right)\right] \\
& \approx \frac{1}{\sqrt{2}}\left(\phi_{\min }+h\right) \exp \left[-i \frac{G}{\left|\phi_{\min }\right|}\right]
\end{aligned}
$$

describes observable physics most appropriately since a vacuum is expected to be at $\phi_{\text {min }}$, instead of the unstable $\phi=0$ point. In fact, the expansion is most conveniently done around only a particular minimum component of the complex doublet

$$
\begin{aligned}
\phi & =\frac{1}{\sqrt{2}}\left(\begin{array}{c}
0+G_{2}+i G_{1} \\
\phi_{\min }+h-i G_{3}
\end{array}\right) \\
& \approx \frac{1}{\sqrt{2}}\left(\begin{array}{c}
0 \\
\phi_{\min }+h
\end{array}\right) \exp \left[-i \vec{\sigma} \cdot \frac{\vec{G}}{\left|\phi_{\min }\right|}\right]
\end{aligned}
$$

whereby the $S U(2)$ symmetry is hidden.

The potential minimum is on the radius degree of freedom assigned to the component field with mass $m_{h}=\sqrt{-2 \mu^{2}}$; the phase degree of freedom corresponds to a massless Goldstone boson demonstrating no energy change for excitation along its direction. The terms of order three or higher in the electroweak Lagrangian equations are for self-couplings of the added scalar field $\phi$.

In a simplified $U(1)$ gauge invariant Lagrangian of electroweak unification

$$
\mathfrak{L}=\left(D_{\mu} \phi\right)^{\dagger}\left(D^{\mu} \phi\right)-\mu^{2} \phi^{\dagger} \phi-\lambda\left(\phi^{\dagger} \phi\right)^{2}-\frac{1}{4} F_{\mu \nu} F^{\mu \nu}
$$




$$
\begin{aligned}
= & \frac{1}{2}\left(\partial_{\mu} h\right)^{2}+\frac{1}{2}\left(\partial_{\mu} G\right)^{2}-\lambda \phi_{\min }{ }^{2} h^{2}+\frac{1}{2} e^{2} \phi_{\min }{ }^{2} A_{\mu} A^{\mu} \\
& -e \phi_{\min } A_{\mu} \partial^{\mu} G-\frac{1}{4} F_{\mu \nu} F^{\mu \nu}+\cdots \\
= & \frac{1}{2}\left(\partial_{\mu} h\right)^{2}-\lambda \phi_{\min }{ }^{2} h^{2}+\frac{1}{2} e^{2} \phi_{\min }{ }^{2} A_{\mu} A^{\mu}-\lambda \phi_{\min } h^{3}-\frac{1}{4} \lambda h^{4} \\
& +\frac{1}{2} e^{2} A_{\mu} A^{\mu} h^{2}+e^{2} \phi_{\min } h A_{\mu} A^{\mu}-\frac{1}{4} F_{\mu \nu} F^{\mu \nu}
\end{aligned}
$$

a vector boson $A_{\mu}$ with mass $m_{A}=e\left|\phi_{\min }\right|$ emerges as the prelude of $W^{ \pm}$and $Z^{0}$ mass generation. The scalar boson with mass $m_{h}=\sqrt{-2 \mu^{2}}$ is called a Higgs particle. The Goldstone boson does not appear as a real particle but it remains as the phase degree of freedom.

In a realistic $S U(2)$ gauge invariant Lagrangian of electroweak unification

$$
\begin{gathered}
\phi \rightarrow \phi^{\prime}=\exp \left[-\frac{i}{2} \vec{f} \cdot \vec{\sigma}\right] \phi \\
D_{\mu}=\partial_{\mu}+\frac{i}{2} g \vec{\sigma} \cdot \vec{W}
\end{gathered}
$$

where

$$
\vec{W}_{\mu} \rightarrow \vec{W}_{\mu}^{\prime}=\vec{W}_{\mu}-\frac{1}{g} \partial_{\mu} \vec{f}-\vec{f} \times \vec{W}
$$

the Lagrangian becomes

$$
\mathfrak{L}=\left(D_{\mu} \phi\right)^{\dagger}\left(D^{\mu} \phi\right)-\left(\mu^{2} \phi^{\dagger} \phi+\lambda\left(\phi^{\dagger} \phi\right)^{2}\right)-\frac{1}{4} \vec{W}_{\mu \nu} \cdot \vec{W}^{\mu \nu}
$$

where $\vec{W}_{\mu \nu}^{(\mu \nu)} \equiv \partial_{\mu}^{(\mu)} \vec{W}_{\nu}^{(\nu)}-\partial_{\nu}^{(\nu)} \vec{W}_{\mu}^{(\mu)}-g \vec{W}_{\mu}^{(\mu)} \times \vec{W}_{\nu}^{(\nu)}$

Inserting Equation (1.47) for the $\phi$ term in Equation (1.54), the relevant Lagrangian terms

$$
\begin{aligned}
\left|\frac{i g}{2} \vec{\sigma} \cdot \vec{W}_{\mu} \phi\right|^{2} & =\frac{g^{2}}{8}\left\|\left(\begin{array}{cc}
W_{3} & W_{1}-i W_{2} \\
W_{1}+i W_{2} & W_{3}
\end{array}\right)\left(\begin{array}{c}
0 \\
\phi_{\min }
\end{array}\right)\right\|^{2} \\
& =\frac{g^{2} \phi_{\min }^{2}}{8}\left(W_{1}^{\dagger} W_{1}+W_{2}^{\dagger} W_{2}+W_{3}^{\dagger} W_{3}\right)
\end{aligned}
$$


bring out vector boson masses for $W^{ \pm}=\frac{1}{\sqrt{2}}\left(W_{1} \mp i W_{2}\right)$ and $Z^{0}=W_{3}$ while keeping the Standard Model renormalizable and gauge invariant.

This mass-giving gauge transformation is called the Higgs mechanism [19]. By way of retaining the gauge invariance of the SM theory and giving $W^{ \pm}$and $Z^{0}$ masses, the Higgs mechanism predicts the existence of a new particle, called the Higgs boson, and sheds light on the origin of fermion masses. Without the Higgs boson, fermions are required to be massless by the SM gauge principle. Parity is always conserved in strong and electromagnetic but not weak interactions. The Higgs boson can generate fermion masses from its VEV by ad hoc coupling to left-handed and right-handed components, $h^{0} \rightarrow f \bar{f}$, Feynman diagram for which is as shown in Figure 1.3. The $S U(2)_{L} \times U(1)_{Y}$ Lagrangian for the electron and its associated neutrino is

$$
\begin{aligned}
\mathfrak{L} & =-G_{e}\left[\left(\bar{\nu}_{e}, e^{+}\right)_{L}\left(\begin{array}{c}
\phi_{a} \\
\phi_{b}
\end{array}\right) e_{R}^{-}+e_{R}^{+}\left(\bar{\phi}_{a}, \bar{\phi}_{b}\right)\left(\begin{array}{c}
\nu_{e} \\
e^{-}
\end{array}\right)\right]_{L} \\
& =-\frac{G_{e}}{\sqrt{2}}\left(e_{L}^{+} e_{R}^{-}+e_{R}^{+} e_{L}^{-}\right) \phi_{\min }-\frac{G_{e}}{\sqrt{2}}\left(e_{L}^{+} e_{R}^{-}+e_{R}^{+} e_{L}^{-}\right) h \\
& =-m_{e} e^{+} e^{-}\left(1+\frac{h}{\phi_{\min }}\right) \text { where } m_{e}=\frac{G_{e}\left|\phi_{\min }\right|}{\sqrt{2}}
\end{aligned}
$$

The $h f \bar{f}$ coupling strength is proportional to the fermion mass.

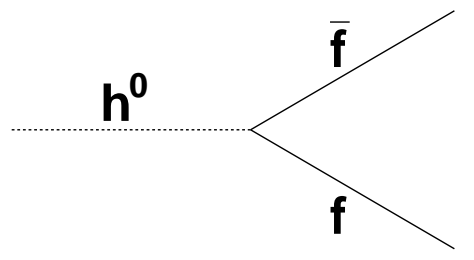

Figure 1.3: Feynman diagram for the Higgs boson coupling to fermions. 


\subsubsection{The Higgs Boson}

Gauge invariance of the Standard Model, through the Higgs mechanism, predicts the existence of a neutral scalar particle, the Higgs boson $h^{0}$ or $H$. Derived from the shape of the scalar $\phi$ field potential, the Higgs boson mass is a free parameter within

loose theoretical bounds, $m_{H}<\frac{8}{3} \sqrt{2} G_{F} \approx 1 \mathrm{TeV}$ by unitarity, as shown in Figure 1.5, and tighter experimental bounds, $114.4<m_{H} \lesssim 200 \mathrm{GeV}$ from LEP and Tevatron, as shown in Figure 2.4. The SM Higgs production cross-sections and decay branching fractions, shown in Figure 2.8 and Figure 2.7, will be discussed in Chapter 2.

Most of the Higgs coupling strengths are clearly specified by theory but not the Higgs self-coupling strengths because self-couplings directly involve the unknown Higgs mass. Good channels to search for the Higgs boson vary with mass, which complicates experimental observation of the Higgs boson.

The Higgs boson couples strongly to heavy particles, such as the $W^{ \pm}$and $Z^{0}$ bosons and the $b$ and $t$ quarks and weakly to light particles, such as the $e$ and $\mu$ leptons and the $u$ and $d$ quarks. In searches for the Higgs boson, the need to produce heavy particles to which the Higgs boson can decay complicates experimental observation of the Higgs boson further.

The Higgs boson is the only SM particle not yet been experimentally confirmed and the search for the Higgs boson is the most important quest for contemporary particle physicists.

\subsubsection{Everything Together and Anything Else}

The Higgs mechanism may answer the puzzle of mass in the context of gauge symmetry. Efforts to perfect the already successful Standard Model have been made 
to unify all forces and to address questions in astrophysics and cosmology. Highlights as follow.

Neutrino masses vanish in the Standard Model outlined above. In fact, the neutrino masses have been limited to $\sum_{\nu} m_{\nu}<1 \mathrm{eV} / \mathrm{c}^{2}$ by present observations of the universe expansion rate.

With non-zero masses, neutrinos are expected and indeed observed to mix and oscillate in ways analogous to quarks in electroweak interactions. Neutrino oscillation explains why the observed solar electron neutrino flux is significantly less than predicted by the solar model that effectively explains all other solar phenomena [21].

A grand unification theory (GUT) to unify electroweak and strong forces has

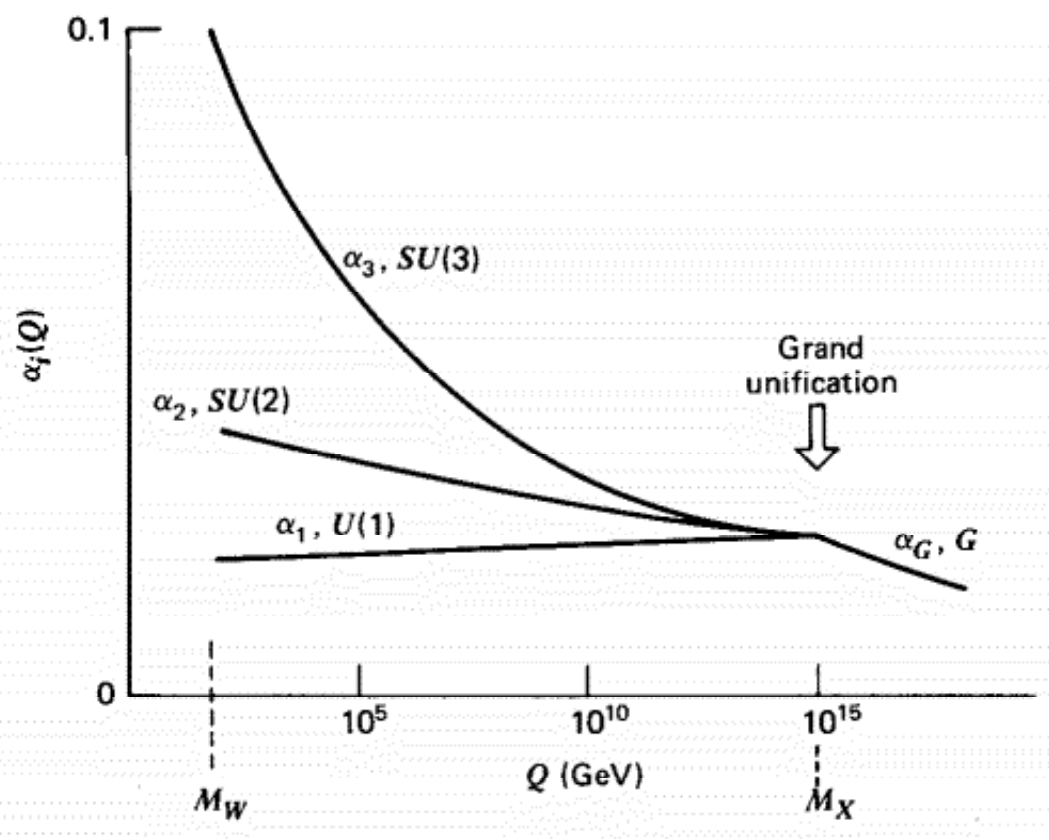

Figure 1.4: Energy scale of electromagnetic $U(1)$, weak $S U(2)$ and strong $S U(3)$ force unification, where all the couplings meet at $m_{X} \sim 10^{15} \mathrm{GeV} / c^{2}[3]$. 
become a natural quest after the magical success of electroweak unification. Georgi and Glashaw proposed the first model [22], predicting that the proton can decay in $10^{30}$ years as well as explaining baryon number asymmetry in the universe with $C P$, $C$ and $B$ violations.

The strong coupling is much stronger than electroweak couplings at the energy scale presently achievable. However, the strong coupling strength decreases with energy-momentum transfer faster than the electroweak coupling strengths. A naive extrapolation suggests that these couplings might meet at rather high energy, as shown in Figure 1.4, assuming nothing unexpected intervenes at any intermediate energy scale. This in term suggests a spontaneous breakdown of a grand symmetry at that energy scale.

The unification of GUT with the gravitational force at the Plank scale $m_{P} \sim$ $10^{19} \mathrm{GeV} / c^{2}$ in a theory of everything (TOE) is still a distant but ultimate goal.

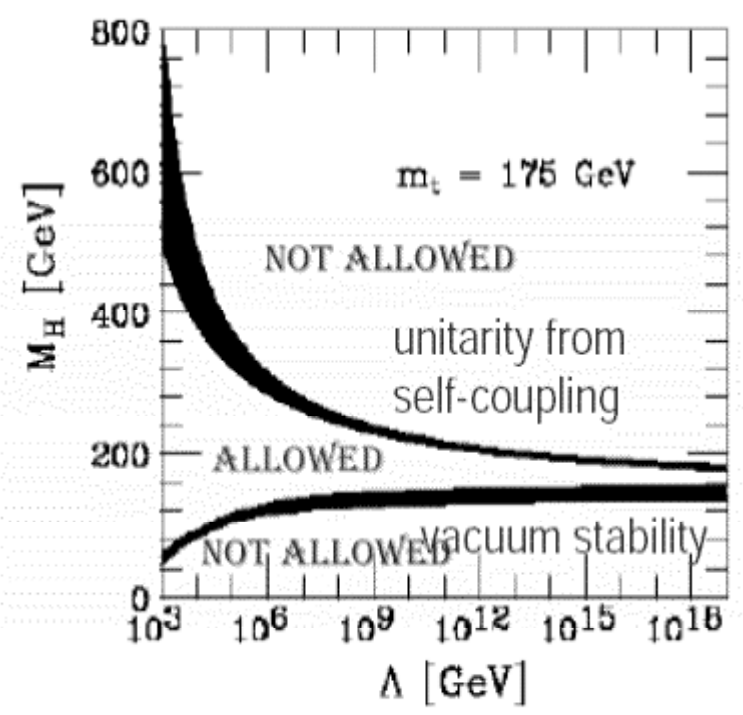

Figure 1.5: Constraints on the Higgs mass as a function of new physics scale [28]. 
Theoretical bounds on the Higgs boson mass as required by vacuum stability and unitarity constraints are correlated with the energy scale of new physics, as shown in Figure 1.5. It may be noted that the Higgs boson mass range pursued by the search documented in this thesis, $m_{H} \sim 160 \mathrm{GeV} / c^{2}$, is consistent with these constraints.

Dark matter is supposed to exist as inferred from observed galactic motions that cannot be accounted for using only luminously observed masses. Dark matter candidates include baryonic massive compact halo objects, such as brown dwarfs and black holes, neutrinos and non-baryonic cold dark matter that likely consists of exotic particles.

\subsection{Beyond the Standard Model}

Exotic models often postulate particle physics that will be revealed at higher energies than ever explored, taking the SM as a low-energy effective theory. Exotic models include extra dimension, little Higgs, technicolor and so forth, with the most popular one being supersymmetry (SUSY) [23].

SUSY is mainly motivated by the cancellation of quadratic divergences in scalar boson mass loop corrections. It postulates that every fermion has a bosonic superpartner and every boson has a fermionic superpartner that has all the same properties except spin. Superparticles can only be produced or annihilated in pairs. The lightest superparticle is stable and hence a good candidate for cold dark matter. Due to

mass, SUSY is an approximate symmetry at best. SUSY is incorporated by GUT and further unification with gravitation at the Plank scale.

A particular model that postulates a heavy 4th Generation of leptons and quarks will be examined in addition to the Standard Model by this analysis. The 
addition of one extra generation of fermions could

1. enhance the Higgs production cross-section

$$
\sigma(g g \rightarrow H)=\frac{G_{F} \alpha_{s}^{2}}{288 \sqrt{2} \pi} \sum_{q}\left|g_{q} A_{q}\right|^{2}
$$

where the coupling strength $g_{q}=m_{q} / \mathrm{VEV}$ and the amplitude $A_{q}$ can be found in [25], as shown in Figure 1.6, because quarks of the extra generation would contribute to the loop mediated process as shown in Figure 2.9,

2. alter the Higgs branching fraction $\operatorname{BR}\left(H \rightarrow W^{+} W^{-}\right)$,

3. relax the Higgs mass upper bound as indicated by electroweak data fits from

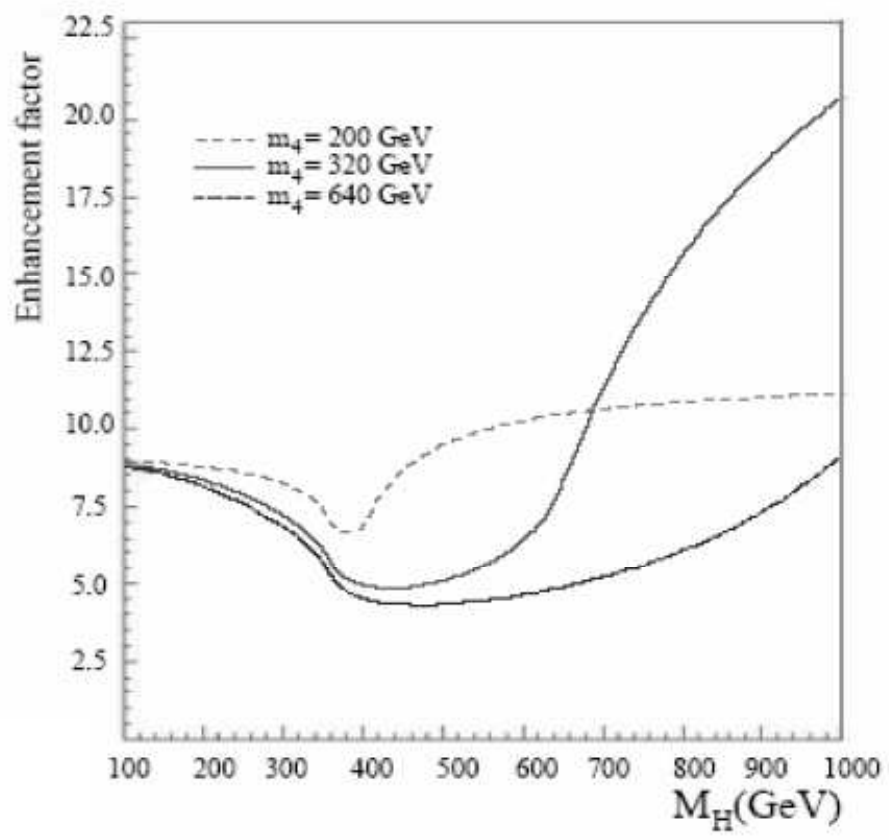

Figure 1.6: Enhancement factors as a function of the Higgs mass for the production cross-section of $\sigma\left(g g \rightarrow h^{0}\right)$ due to the addition of one extra generation of fermions [24]. The dip corresponds to the top quark pair mass. 
$200 \mathrm{GeV}$ to $500 \mathrm{GeV}$.

Direct searches for fermions of any extra family have set the following mass limits: $m_{\ell_{4}}>92.4 \mathrm{GeV}, m_{\nu_{4}}>45 \mathrm{GeV}$ and $m_{d_{4}}>199 \mathrm{GeV}$. 


\section{Chapter 2}

\section{Searches for the SM Higgs Boson}

The Higgs boson has been searched for using various methods in many experiments since it has been predicted to be the origin of mass by gauge invariance in the Standard Model. Experimental confirmation of the Higgs boson's existence is the most important quest for contemporary particle physicists and, in one channel, it is the topic of analysis this thesis.

\subsection{Fits with Electroweak Data}

Precision measurement results from the large electron-positron collider (LEP) experiments on electroweak parameters, such as cross-sections, masses and couplings, have been combined using ZFITTER 6.42 [26] by the LEP electroweak working group (EWWG) at CERN and further combined with the results from other experiments, including CDF, DØ, NuTeV and SLD, by the Tevatron EWWG, compared to theories and published every half a year. So far the minimal SM (MSM) describes all the experimental results and new theories are not needed.

Particular interest in the EWWG global MSM fit results is given to parameters pertaining to the SM Higgs boson, especially the $W$ boson mass $m_{W}$ and the top 
quark mass $m_{t}$ which have been used extensively to infer the Higgs boson mass $m_{H}$.
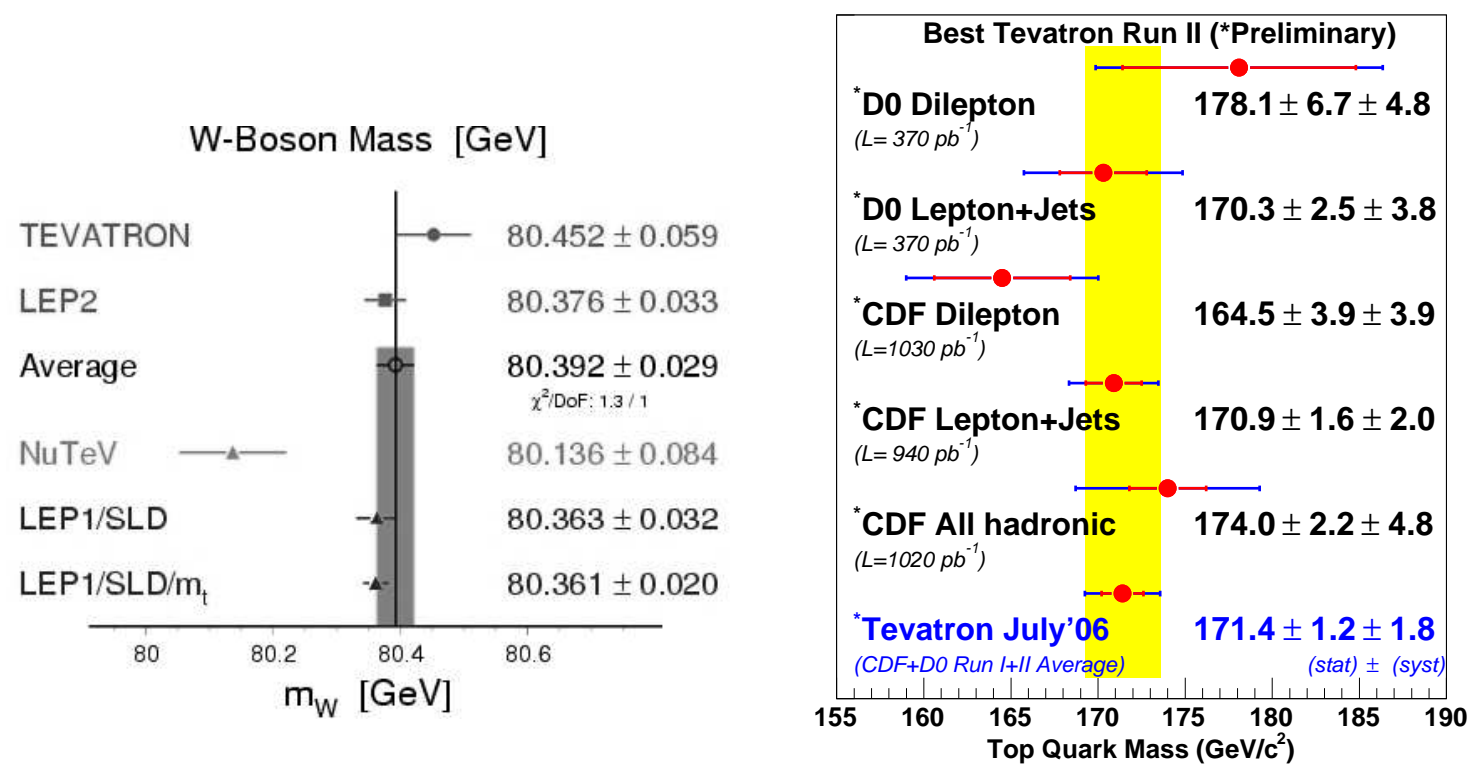

Figure 2.1: Summary of the latest precision measurement results of the $W$ boson mass, including one from the $\mathrm{NuTeV}$ neutrino-nucleon scattering experiment, which is 2.62.8 standard deviations away from the other results (left). Summary of the latest precision measurement results of the top quark mass (right).

The latest experimental $W$ boson mass and top quark mass measurement results are summarized in Figure 2.1. Contours of $m_{W}$ vs. $m_{t}$ prefer a low Higgs boson mass, as shown with the $m_{H}$ grid in Figure 2.2.

The $\Delta \chi^{2}$ of the global MSM fit as a function of the Higgs mass, derived from the latest precision electroweak measurement results as shown in Figure 2.3, is shown in Figure 2.4 assuming the Standard Model is correct up to any energy scale. The preferred Higgs mass with experimental uncertainties is $85_{-28}^{+39} \mathrm{GeV} / c^{2}$ at $68 \%$ confidence level (CL), corresponding to the solid-lined $\Delta \chi^{2}$ minimum in Figure 2.4; the minimum is marginally affected by the low- $Q^{2}$ results. The Higgs mass is predicted to 


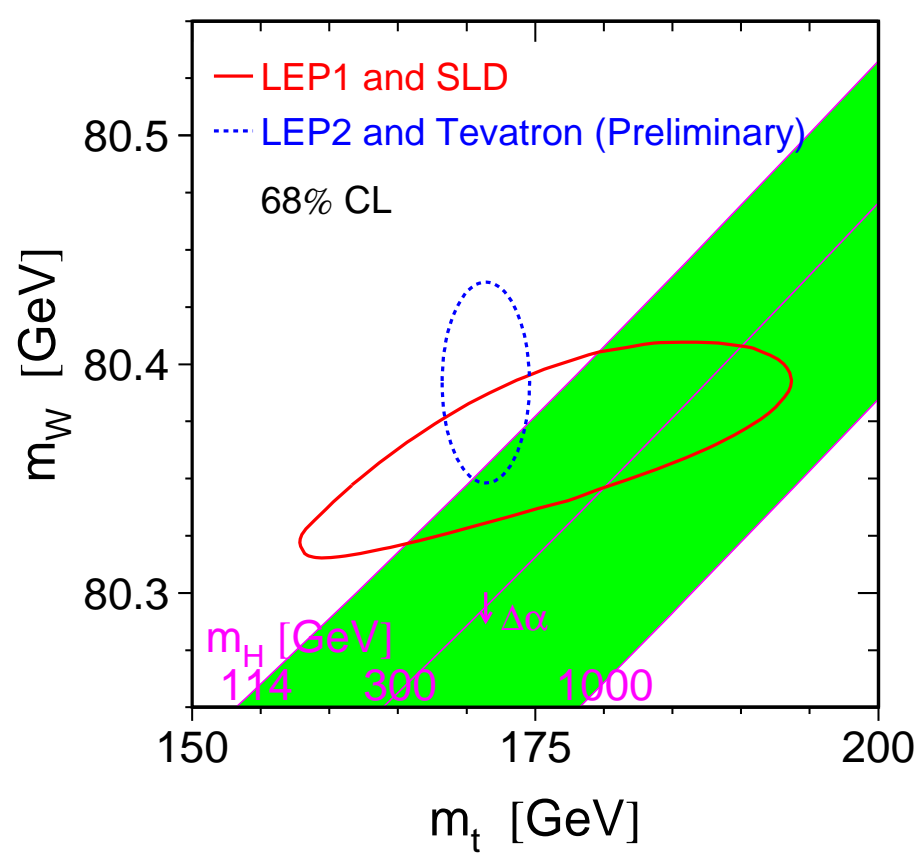

Figure 2.2: Contours of the $W$ boson mass vs. the top quark mass on the Higgs boson mass grid.

\begin{tabular}{|c|c|c|c|}
\hline & Measurement & Fit & $\mathrm{O}^{\text {meas }}-\mathrm{O}^{\text {fit }} \mid / \sigma^{\text {meas }}$ \\
\hline$\Delta \alpha_{\text {had }}^{(5)}\left(m_{z}\right)$ & $0.02758 \pm 0.00035$ & 0.02766 & 1 \\
\hline$m_{z}[\mathrm{GeV}]$ & $91.1875 \pm 0.0021$ & 91.1874 & \\
\hline$\Gamma_{\mathrm{Z}}[\mathrm{GeV}]$ & $2.4952 \pm 0.0023$ & 2.4957 & $\mathbf{P}$ \\
\hline$\sigma_{\text {had }}^{0}[n b]$ & $41.540 \pm 0.037$ & 41.477 & \\
\hline $\mathrm{R}_{\mathrm{I}}$ & $20.767 \pm 0.025$ & 20.744 & \\
\hline$A_{\mathrm{fb}}^{0, I}$ & $0.01714 \pm 0.00095$ & 0.01640 & \\
\hline $\mathrm{A}_{\mid}\left(\mathrm{P}_{\tau}\right)$ & $0.1465 \pm 0.0032$ & 0.1479 & 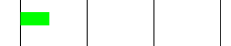 \\
\hline $\mathrm{R}_{\mathrm{b}}$ & $0.21629 \pm 0.00066$ & 0.21585 & \\
\hline $\mathrm{R}_{\mathrm{c}}$ & $0.1721 \pm 0.0030$ & 0.1722 & 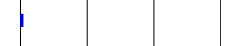 \\
\hline$A_{\mathrm{fb}}^{0, b}$ & $0.0992 \pm 0.0016$ & 0.1037 & \\
\hline $\mathrm{A}_{\mathrm{fb}}^{0, \mathrm{c}}$ & $0.0707 \pm 0.0035$ & 0.0741 & \\
\hline$A_{b}$ & $0.923 \pm 0.020$ & 0.935 & 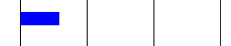 \\
\hline$A_{c}$ & $0.670 \pm 0.027$ & 0.668 & 1 \\
\hline$A_{1}(S L D)$ & $0.1513 \pm 0.0021$ & 0.1479 & \\
\hline $\sin ^{2} \theta_{\text {eff }}^{\text {lept }}\left(Q_{\mathrm{fb}}\right)$ & $0.2324 \pm 0.0012$ & 0.2314 & 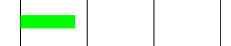 \\
\hline $\mathrm{m}_{\mathrm{W}}[\mathrm{GeV}]$ & $80.392 \pm 0.029$ & 80.371 & 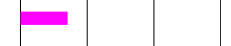 \\
\hline$\Gamma_{\mathrm{W}}[\mathrm{GeV}]$ & $2.147 \pm 0.060$ & 2.091 & \\
\hline \multirow[t]{2}{*}{$m_{t}[\mathrm{GeV}]$} & $171.4 \pm 2.1$ & 171.7 & 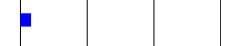 \\
\hline & & & 1 \\
\hline
\end{tabular}

Figure 2.3: Pull of the EWWG's global MSM fit. 
be below $199 \mathrm{GeV} / c^{2}$, or $166 \mathrm{GeV} / c^{2}$ ignoring the LEP II direct search result, at $95 \%$ CL by the fit, taking into account both theoretical and experimental uncertainties.

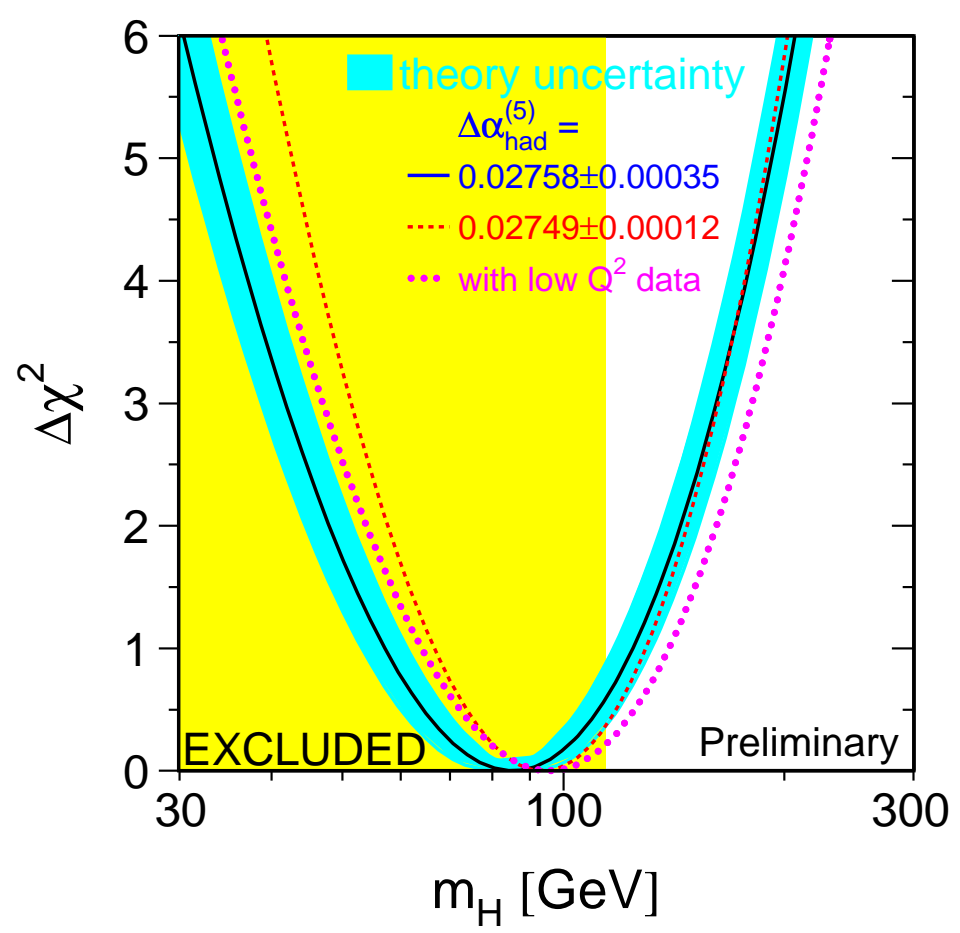

Figure 2.4: $\Delta \chi^{2}$ of the global MSM fit as a function of the Higgs mass (foreground) and exclusion by LEP II direct searches (background). It is labeled "preliminary" because, even though the LEP II electroweak measurement results were all final, the combination used a preliminary LEP II estimate for color reconnection effect.

\subsection{Direct Searches at LEP II}

At LEP the SM Higgs boson was directly searched for in $2461 \mathrm{pb}^{-1} e^{+} e^{-}$collisions at $\sqrt{s}=189-209 \mathrm{GeV}[27]$, mainly via $e^{+} e^{-} \rightarrow Z^{*} \rightarrow Z H$ (Higgsstrahlung) channels.

At $m_{H}=115 \mathrm{GeV}$, the primary SM Higgs decay channel and secondary ones are $H \rightarrow b \bar{b}(\mathrm{BR}=74 \%)$ and $H \rightarrow\left\{\tau^{+} \tau^{-}(7 \%), W^{+} W^{-}(7 \%), g g(7 \%), c \bar{c}(4 \%) \ldots\right\}$. The $Z$ 
boson decay channels are available in Table 1.5. The search topologies included $(Z \rightarrow$ $\left.\left\{\ell^{+} \ell^{-}, \nu \bar{\nu}, q \bar{q}\right\}\right)(H \rightarrow b \bar{b})$ and $(Z \rightarrow q \bar{q})\left(H \rightarrow \tau^{+} \tau^{-}\right)$. Background primarily came from diphoton processes and radiative returns to the $Z$ boson and secondarily from the $W W, Z Z, f \bar{f}$ and $\gamma / g$-radiative processes. Identification of $b$ jets and reconstruction of the Higgs mass played important roles in signal discrimination against background.

To combine the searches in different channels at different center-of-mass energies from different experiments, each search was binned in two variables: $m_{H}^{r e c}$ for the reconstructed Higgs boson mass and $\mathcal{G}$ that combined analysis-dependent event features such as $b$-tagging variables, likelihood functions or neural network outputs, allowing

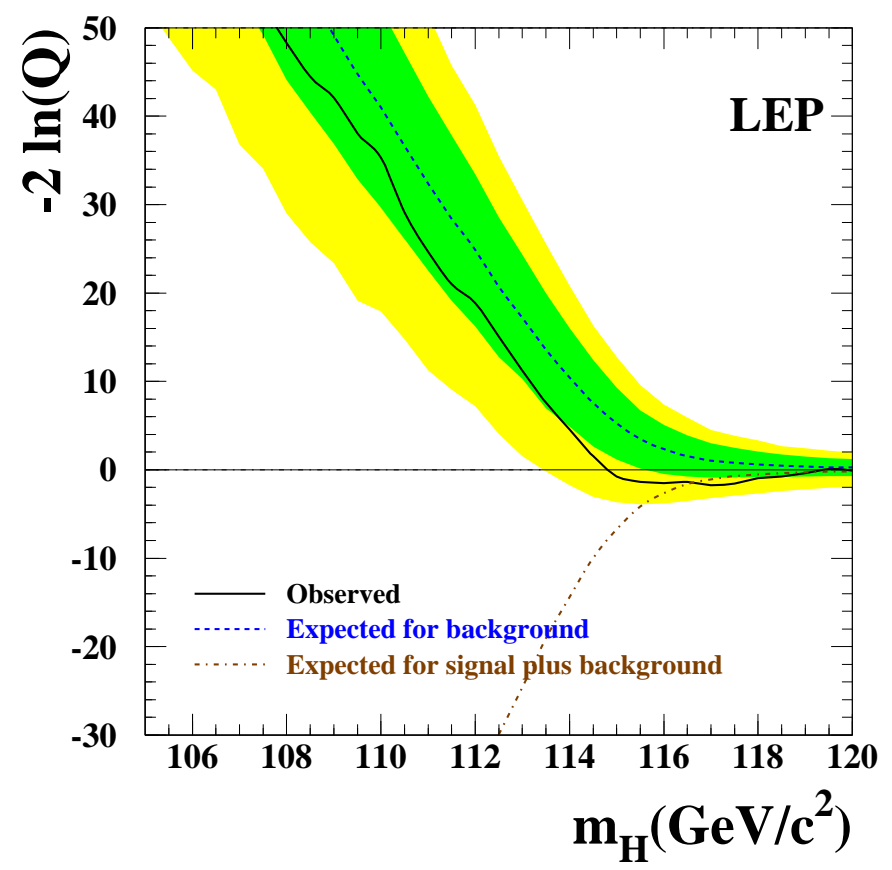

Figure 2.5: Test statistic $-2 \ln Q$ as a function of the Higgs mass, combining all the LEP SM Higgs boson searches [27]. Shades that accompany the curve for the expected background represent the $95 \%$ (inner) and $68 \%$ (outer) probability bands about the median of the expected background. 
discrimination on a statistical basis. For each channel and $\left(m_{H}^{r e c}, \mathcal{G}\right)$ bin, number of observed data events, expected signal and expected background were provided for a set of hypothetical Higgs masses. The observed data configuration in the $\left(m_{H}^{r e c}, \mathcal{G}\right)$ plane was subjected to a likelihood ratio

$$
Q \equiv \frac{\operatorname{Pr}(s+b)}{\operatorname{Pr}(b)}
$$

test of two hypothetical scenarios: $b$ for background only and $s+b$ for signal plus background. In their initial full dataset analyses, ALEPH observed an excess consistent with the SM Higgs production at mass $m_{H}=115 \mathrm{GeV}$; L3 and OPAL slightly favored the $s+b$ hypothesis around $m_{H}=115 \mathrm{GeV}$ while being consistent with the $b$ hypothesis; DELPHI reported a slight deficit with respect to background expectation.

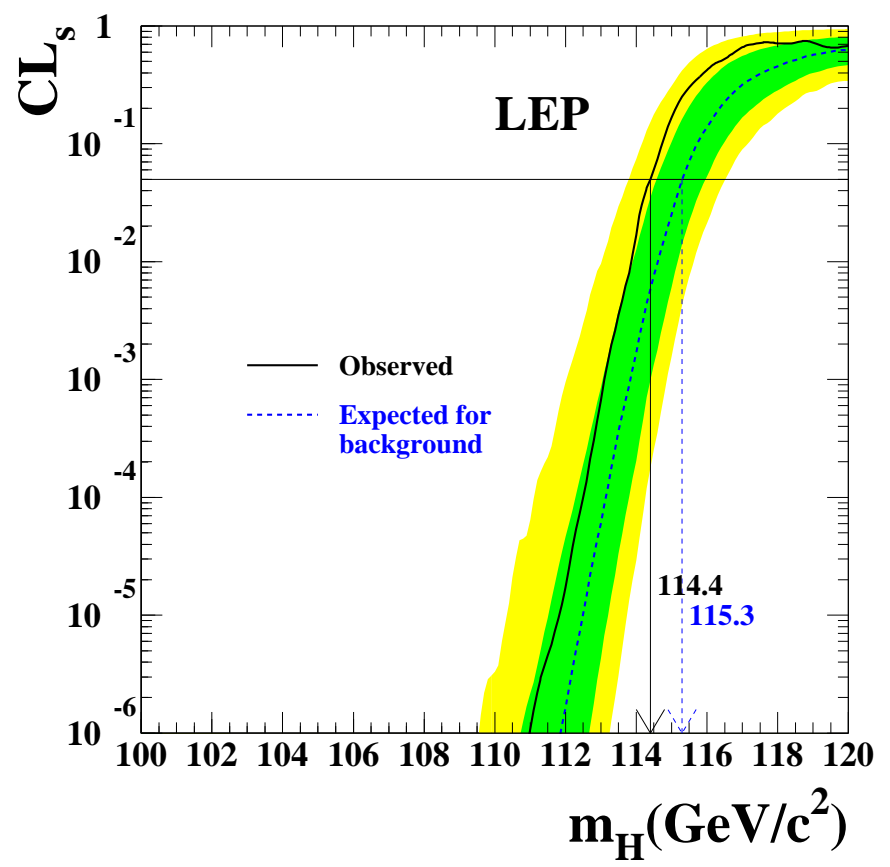

Figure 2.6: Signal confidence $\mathrm{CL}_{s}$ as a function of the Higgs mass, combining all the LEP SM Higgs boson searches [27]. The SM Higgs mass $m_{H} \geq 114.4 \mathrm{GeV}$ was observed while $m_{H} \geq 115.3 \mathrm{GeV}$ was expected at $95 \% \mathrm{CL}$. 
The LEP-combined test statistic $-2 \ln Q$ as a function of the Higgs mass is shown in Figure 2.5; there is a broad minimum around $m_{H}=115 \mathrm{GeV} / c^{2}$ where the solid curve for what was observed in data goes into the negative $-2 \ln Q$ region and favors the $s+b$ hypothesis with low significance.

Integrating the probability distribution function for the $b$ hypothesis from the observed value to $+\infty$, the background confidence $1-\mathrm{CL}_{b}$ was obtained to express the compatibility of observations with the $b$ hypothesis. Similarly, integrating the probability distribution function for the $s+b$ hypothesis from $-\infty$ to the observed value, the signal-plus-background confidence $\mathrm{CL}_{s+b}$ was obtained to express the compatibility of observations with the $s+b$ hypothesis. The signal confidence, defined as

$$
\mathrm{CL}_{s} \equiv \frac{\mathrm{CL}_{s+b}}{\mathrm{CL}_{b}}
$$

was used to derive a lower bound on the SM Higgs mass, in which the test mass giving $\mathrm{CL}_{s}=0.05$ was taken as the lower limit at $95 \% \mathrm{CL}$. The LEP-combined $\mathrm{CL}_{s}$ as a function of the Higgs mass is shown in Figure 2.6.

\subsection{Direct Searches at Tevatron}

At the Tevatron the Higgs boson has been directly searched for via various channels in $p \bar{p}$ collisions at $\sqrt{s}=1.8-1.96 \mathrm{TeV}$.

Among the SM Higgs production modes at the Tevatron, as shown in Figure 2.7,

1. gluon-gluon fusion through a quark loop has the largest cross-section for any Higgs mass, $1.17<\sigma_{\mathrm{SM}}(g g \rightarrow H)<0.145 \mathrm{pb}$ for $100<m_{H}<200 \mathrm{GeV}$;

2. vector boson associated (Higgsstrahlung) productions have cross-sections $\sigma(q q \rightarrow$ $\left.W^{*} \rightarrow W H\right) \sim \frac{1}{3} \sigma(g g \rightarrow H)$ and $\sigma\left(q \bar{q} \rightarrow Z^{*} \rightarrow Z H\right) \sim \frac{1}{6} \sigma(g g \rightarrow H) ;$ 


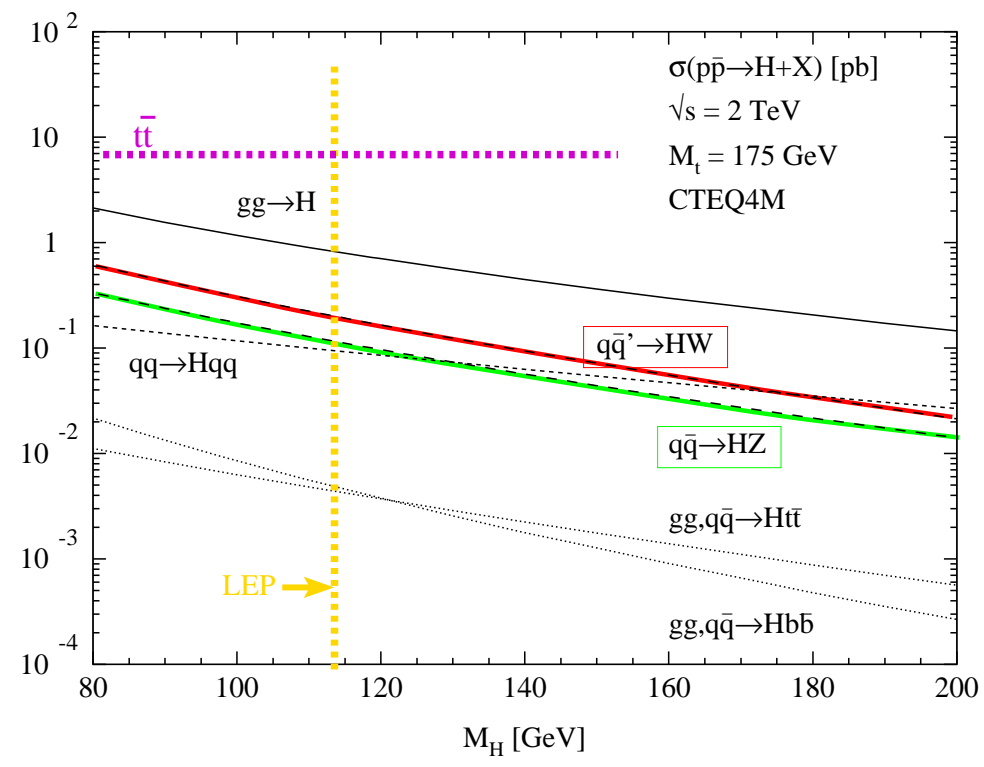

Figure 2.7: Cross-sections of the dominant SM Higgs production modes at the Tevatron.

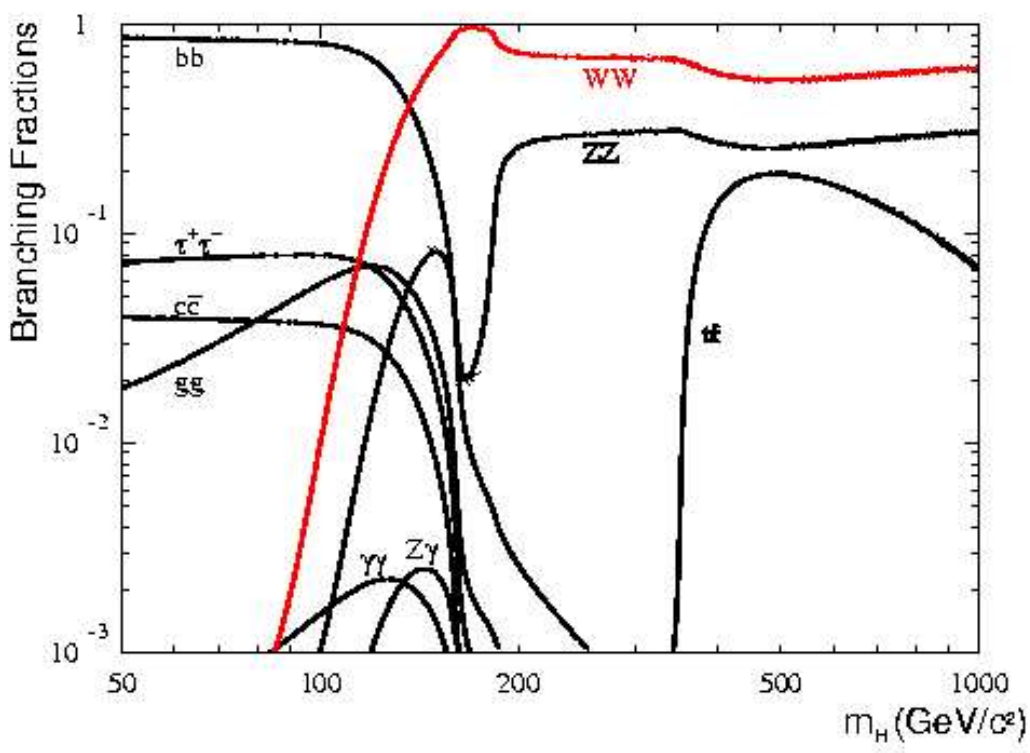

Figure 2.8: Decay branching fractions of the Higgs boson in the Standard Model. 
3. vector boson fusion (VBF), $q q \rightarrow V^{*} q V^{*} q \rightarrow H q q$, has its cross-section comparable to the Higgsstralung ones but decreasing slowly as the Higgs mass increases. The production cross-section of VBF emulates $Z H$ at $m_{H} \sim 130 \mathrm{GeV}$ and $W H$ at $m_{H} \sim 175 \mathrm{GeV}$.

As shown in Figure 2.8, in the low mass region $\left(m_{H} \lesssim 135 \mathrm{GeV}\right)$ the SM Higgs boson decays to $b \bar{b}(\mathrm{BR} \sim 80 \%), c \bar{c}(\mathrm{BR} \sim 4 \%), \tau^{+} \tau^{-}(\mathrm{BR} \sim 7 \%), W^{+} W^{-}$and $g g$ (BRs varying). In the high mass region $\left(m_{H} \gtrsim 135 \mathrm{GeV}\right)$ the SM Higgs boson predominantly decays to a vector boson pair, $W W(\mathrm{BR} \sim 90 \%)$ and $Z Z(\mathrm{BR} \lesssim 10 \%)$.

In the low Higgs mass region, preferred by the global MSM fit, direct searches in the Higgsstrahlung channels are more promising, although the cross-sections may be smaller than in other channels, because leptonic decays of the associated vector boson can be used to suppress the QCD background that accompanies $H \rightarrow b \bar{b}$ selection.

In the high Higgs mass region, however, direct searches in the gluon-gluon fusion or even VBF channels are superior because the cross-sections are large and leptonic

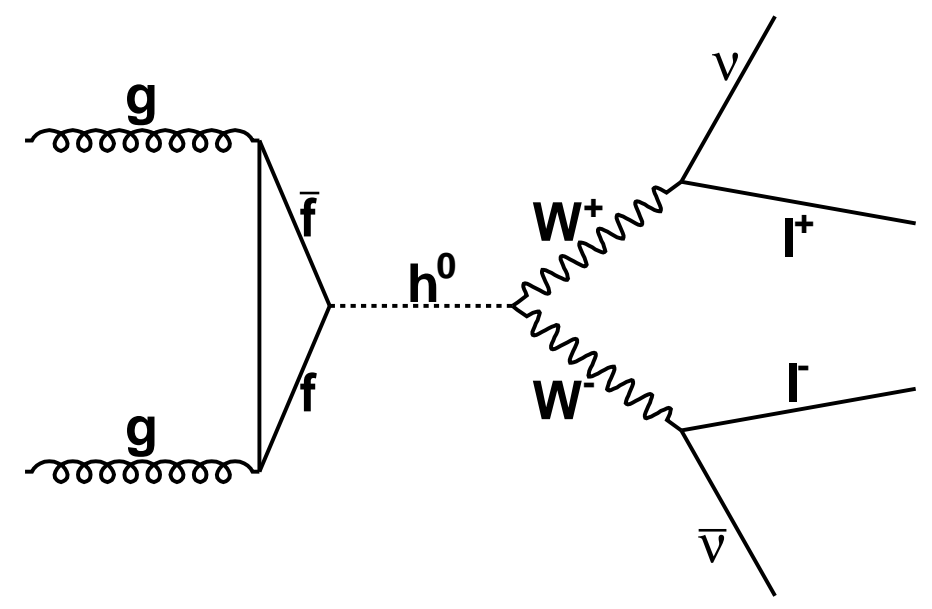

Figure 2.9: Feynman diagram for the major production and dileptonic decay of $H W W$ at the Tevatron, $g g \rightarrow h^{0} \rightarrow W^{+} W^{-} \rightarrow \ell^{+} \nu_{\ell} \ell^{-} \bar{\nu}_{\ell}$. 
decays of the daughter vector bosons of the Higgs boson can be used to avoid the QCD background. The channel $g g \rightarrow h^{0} \rightarrow W^{+} W^{-} \rightarrow \ell^{+} \nu_{\ell} \ell^{-} \bar{\nu}_{\ell}$, for which the Feynman diagram is shown in Figure 2.9, is most sensitive in the high Higgs mass range since it combines the largest production cross-section, the largest Higgs decay branching ratio and the highest signal-to-background ratio.

Both CDF and DØ strive to improve all the factors in direct Higgs searches, including

1. rapidity coverage of lepton and $b$-tagging;

2. $b$-tagging efficiency and mistag rate;

3. $b$-specific jet correction, which involves muon and missing energy;

4. dijet mass resolution;

5. neural network;

6. search channel coverage;

7. result combination.

All the improvements are in addition to the desperate need for integrated luminosity to reduce systematic uncertainty $\frac{1}{\sqrt{\mathcal{L}_{i n t}}}$ proportionally.

The discovery, evidence and exclusion potential of direct searches for the SM Higgs boson at the Tevatron as a function of the Higgs mass and integrated luminosity, estimated at the beginning of Run II in 2003, is shown in Figure 2.10. Direct searches for the SM Higgs boson are a challenge to the Tevatron. 


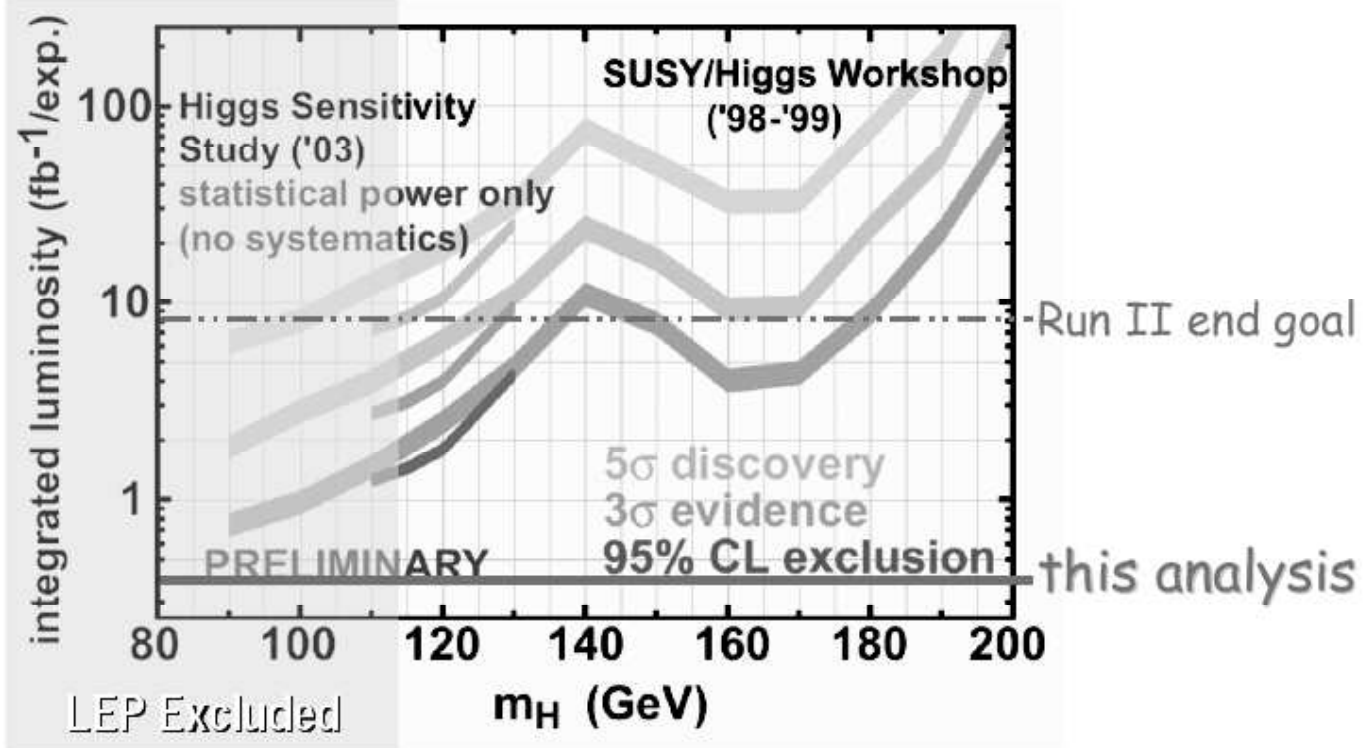

Figure 2.10: The discovery, evidence and exclusion potentials by direct searches for the SM Higgs boson at the Tevatron, estimated in 2003 [28]. The integrated luminosity is per experiment.

\subsubsection{Run I}

CDF searched for the SM Higgs boson in $p \bar{p}$ collisions at $\sqrt{s}=1.8 \mathrm{TeV}$ via vector boson associated Higgs production channels, using up to $106 \pm 4 \mathrm{pb}^{-1}$ Run I data collected from 1992 to 1995 [29].

Three $b$-tagging algorithms were used for $H \rightarrow b \bar{b}$ identification: secondary vertex (secvtx), soft lepton and jet probability. All the analyses required the presence of at least one $b$ tagged by secvtx, which had the highest tagging efficiency among all the algorithms. The signal-to-background ratio was greatly improved by requiring the presence of the associated vector boson decay daughters. A resonance peak at $m_{H}$ in dijet mass spectrum was looked for.

Results from individual analyses are summarized in Table 2.1. The $Z H \rightarrow$ 


\begin{tabular}{|l|c|ccc|c|c|c|}
\hline \hline \multirow{2}{*}{$\begin{array}{l}\text { searched } \\
\text { channel }\end{array}$} & $N_{b}$ & \multicolumn{3}{|c|}{ acceptance (\%) for } & background & observed & $\mathcal{L}_{\text {int }}$ \\
tags & $m_{H}=90$, & 110, & $130(\mathrm{GeV})$ & expectation & events & $\left(\mathrm{pb}^{-1}\right)$ \\
\hline \hline \multirow{2}{*}{$W H \rightarrow \ell \nu b \bar{b}$} & 1 & $0.55 \pm 0.14$ & $0.74 \pm 0.18$ & $0.89 \pm 0.22$ & $30 \pm 5$ & 36 & 106 \\
\cline { 2 - 8 } & 2 & $0.23 \pm 0.06$ & $0.29 \pm 0.07$ & $0.34 \pm 0.09$ & $3.0 \pm 0.6$ & 6 & 106 \\
\hline$V H \rightarrow q q b \bar{b}$ & 2 & $1.3 \pm 0.4$ & $2.2 \pm 0.6$ & $3.1 \pm 0.8$ & $594 \pm 30$ & 589 & 91 \\
\hline$Z H \rightarrow \ell \ell b \bar{b}$ & $1+2$ & $0.14 \pm 0.03$ & $0.20 \pm 0.04$ & $0.19 \pm 0.04$ & $3.2 \pm 0.7$ & 5 & 106 \\
\hline$Z H \rightarrow \nu \bar{\nu} b \bar{b}$ & 1 & $0.59 \pm 0.12$ & $0.69 \pm 0.14$ & $0.86 \pm 0.17$ & $39.2 \pm 4.4$ & 40 & 88 \\
\cline { 2 - 8 } & 2 & $0.37 \pm 0.08$ & $0.44 \pm 0.11$ & $0.53 \pm 0.11$ & $3.9 \pm 0.6$ & 4 & 88 \\
\hline \hline
\end{tabular}

Table 2.1: Summary of the CDF Run I SM Higgs searches, including signal acceptance, background expectation and data observation.

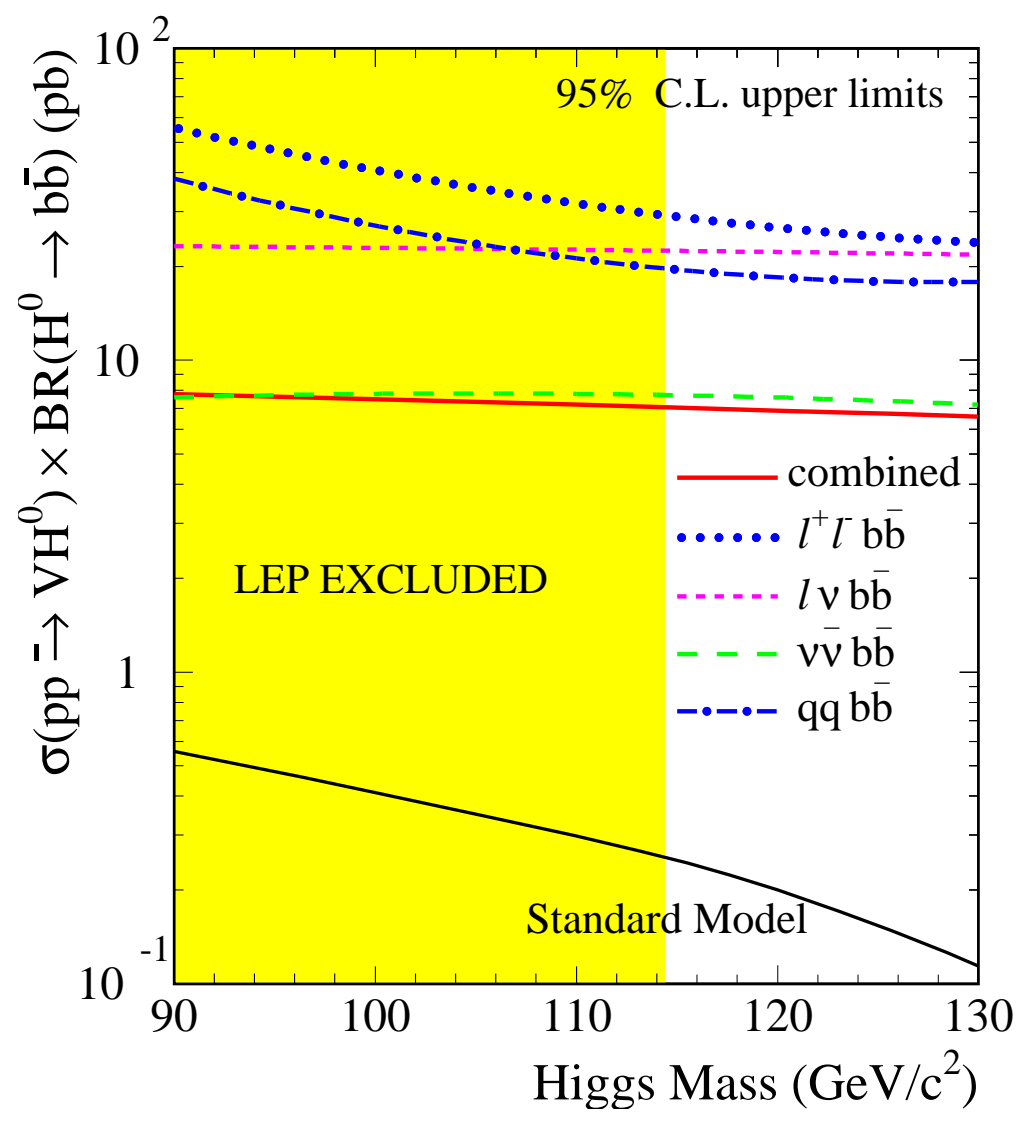

Figure 2.11: Results of the CDF Run I direct searches for the SM Higgs boson, i.e. the upper limits on the vector boson associated Higgs production cross-sections at the Tevatron in Run I. 
$\nu \bar{\nu} b \bar{b}$ channel was dominant. Result combination was performed with the product of individual likelihoods binned in the dijet mass spectrum. The 95\% CL upper limit on the vector boson associated SM Higgs production cross-section, modified by the branching fraction of Higgs decay to a $b$ quark pair, was set to

$$
\begin{aligned}
& \sigma(p \bar{p} \rightarrow V H) \times \mathrm{BR}(H \rightarrow b \bar{b})<8.2 \mathrm{pb} \text { for } m_{H}=90 \mathrm{GeV} / c^{2} \\
& \sigma(p \bar{p} \rightarrow V H) \times \mathrm{BR}(H \rightarrow b \bar{b})<7.8 \mathrm{pb} \text { for } m_{H}=110 \mathrm{GeV} / c^{2} \\
& \sigma(p \bar{p} \rightarrow V H) \times \mathrm{BR}(H \rightarrow b \bar{b})<7.4 \mathrm{pb} \text { for } m_{H}=130 \mathrm{GeV} / c^{2}
\end{aligned}
$$

and summarized in Figure 2.11. The limits were a factor of 15-50 away from the SM prediction.

\subsubsection{Run II}

Both CDF and DØ have worked fervently on searches for the Higgs boson in Run II. Results via various channels are being updated from time to time along with the increase of integrated luminosity.

This thesis focuses on a direct search for the SM Higgs boson in the $p \bar{p} \rightarrow H \rightarrow$ $W^{+} W^{-} \rightarrow \ell^{+} \nu_{\ell} \ell^{-} \bar{\nu}_{\ell}(H W W$ dilepton$)$ channel - the first search at CDF. A similar search was performed by the D $\varnothing$ collaboration [30].

The Higgs search experience at the Tevatron will be valuable to the large hadron collider (LHC) at CERN, of which the construction is close to complete and the operation is close to starting. 


\section{Chapter 3}

\section{Tevatron and the CDF II Detector}

The Tevatron is the world's highest energy particle collider to date. It collides proton $p$ and antiproton $\bar{p}$ beams at center-of-mass energy $E_{c m}=1.96 \mathrm{TeV}$ in a typical

scale of instantaneous luminosity $10^{30-32} \mathrm{~cm}^{-2} \mathrm{~s}^{-1}$. It provides two collider detector experiments at Fermilab (CDF and DØ) with incommensurable particle physics research potential. The CDF and $\mathrm{D} \varnothing$ experiments are designed for general purposes with research directions encompassing five complementary lines of attack on the open questions of the Standard Model:

1. the characterization of top quark properties;

2. global electroweak precision program;

3. direct searches for new phenomena, including the Higgs boson;

4. tests of perturbative QCD at next-to-leading order (NLO) and large $Q^{2}$;

5. the constraint of the CKM matrix with high statistics hadron decays.

The CDF and DØ experiments are internationally funded collaborations of approximately 700 physicists each. Fermilab is mainly funded by the Department of Energy 


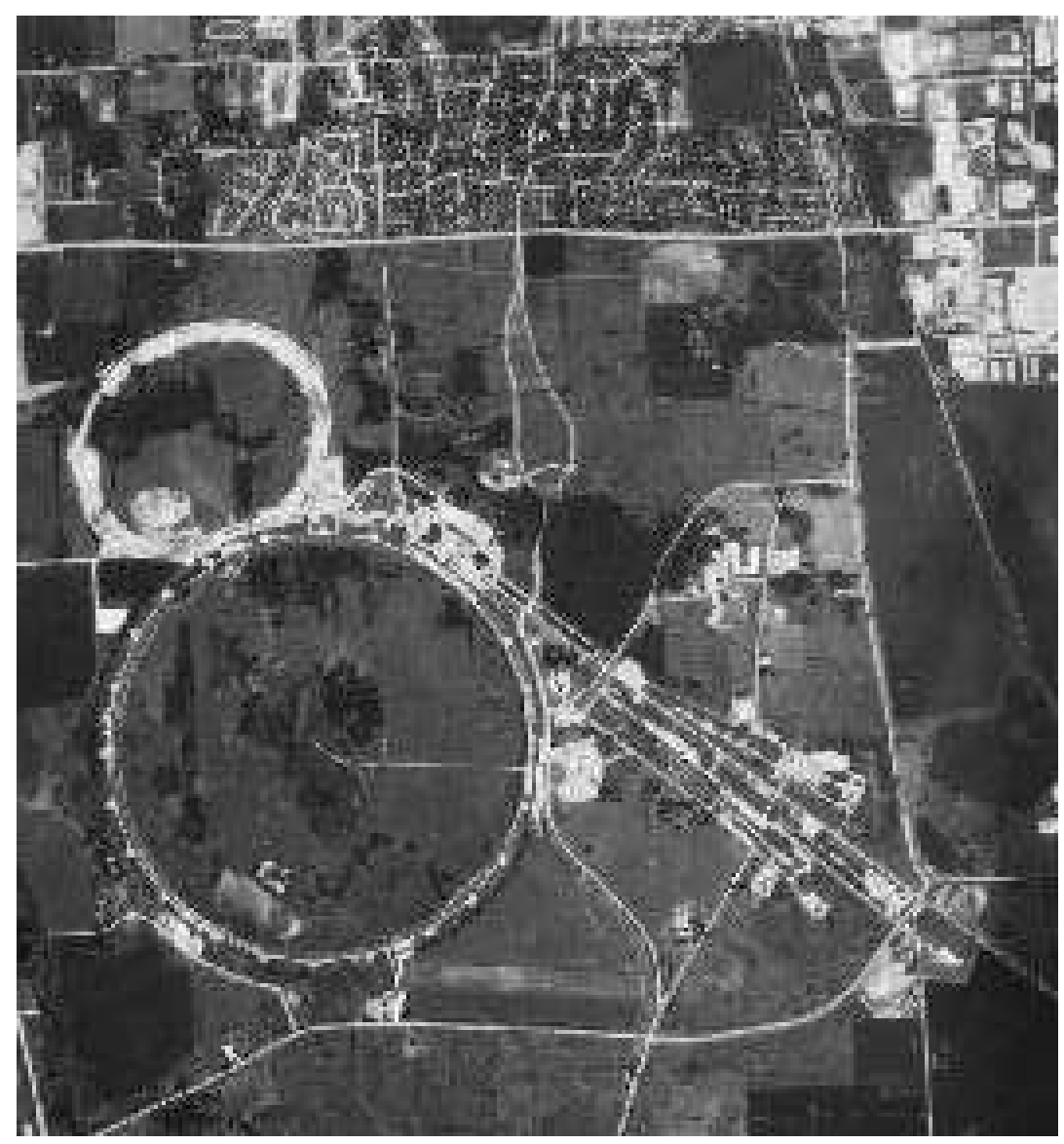

Figure 3.1: Aerial photograph of Fermilab.

of the United States (US DoE).

\subsection{Accelerator Complex at Fermilab}

The Tevatron is located at Fermilab as the final stage of an accelerator chain, as shown in Figure 3.1 and Figure 3.2. The accelerator chain at Fermilab consists of the following six stages:

1. The Cockcroft-Walton machine provides the first stage of acceleration. Inside this device, hydrogen gas is ionized to create negative ions $H^{-}$, each consisting of two electrons and one proton. The $H^{-}$ions are accelerated by a positive 


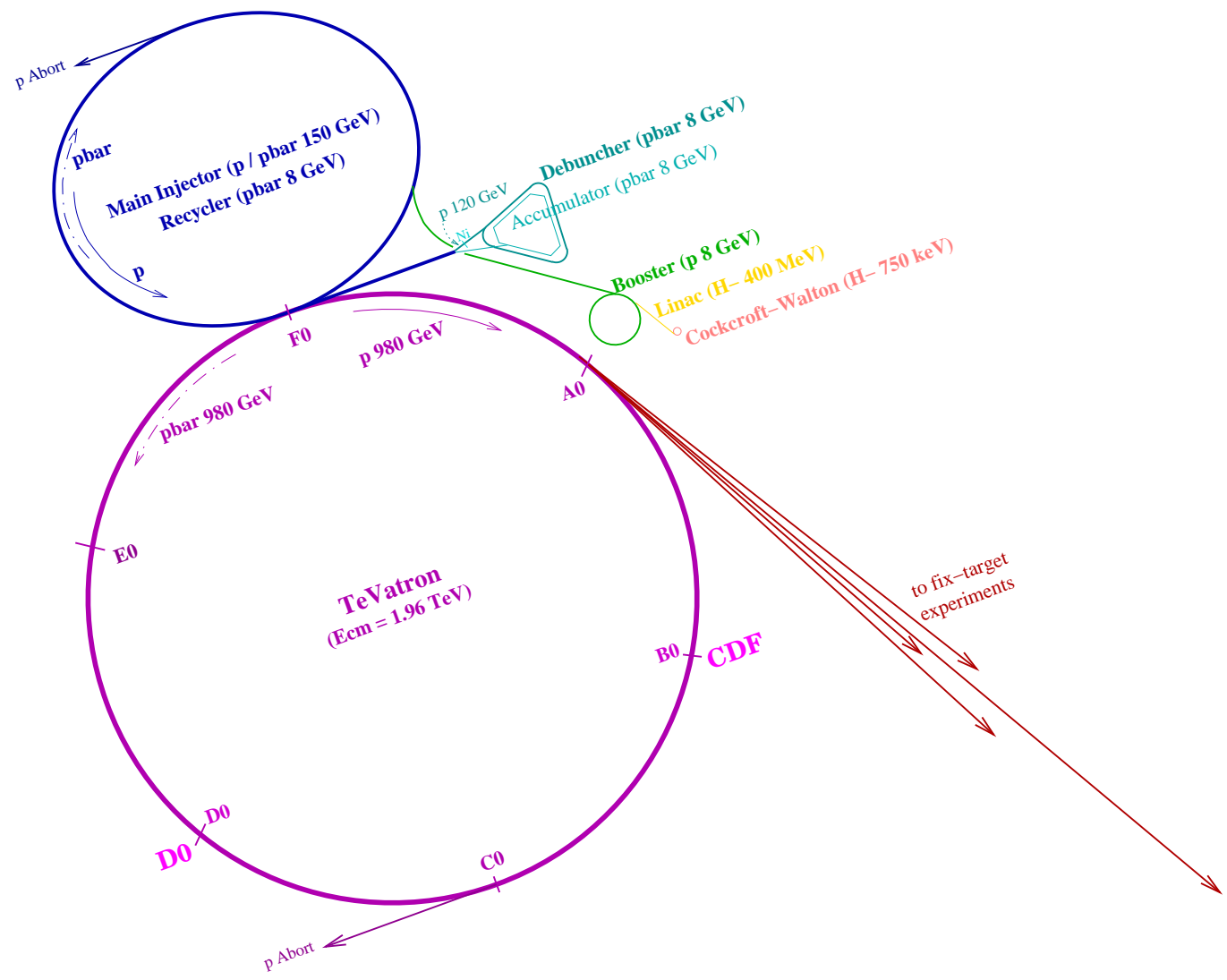

Figure 3.2: Illustration of the Fermilab accelerator complex. 
voltage to reach $750 \mathrm{keV}$.

2. The $H^{-}$ions enter a linear accelerator, called the Linac, that is $500 \mathrm{ft}$ long, get accelerated by oscillating electric fields to $400 \mathrm{MeV}$ and then pass through a carbon foil to get rid of electrons so that only protons leave for the next stage.

3. The protons enter the Booster, bent by magnets to move in a circular orbit and accelerated to $8 \mathrm{GeV}$ in 20,000 revolutions.

4. The protons enter the Main Injector (MI) that

(a) receives protons from the Booster and accelerates them up to $150 \mathrm{GeV}$;

(b) extracts $120 \mathrm{GeV}$ protons to the Antiproton Source (AS);

(c) receives antiprotons from the $\mathrm{AS}$ and accelerates them to $150 \mathrm{GeV}$;

(d) injects $150 \mathrm{GeV}$ protons and antiprotons into the Tevatron.

5. The protons extracted from the MI to the AS hit a nickel target and produce antiprotons out of a broad spectrum of secondary particles. The antiprotons are collected, focused and stored in the Accumulator. When accumulated to a sufficient number, the antiprotons are injected at $8 \mathrm{GeV}$ back into the $\mathrm{MI}^{1}$.

6. The protons and antiprotons, accelerated by the MI to $150 \mathrm{GeV}$, enter the Tevatron in opposite directions and get accelerated to $980 \mathrm{GeV}$. The beams cross each other at the B0 and D0 points, which are respectively the CDF and D $\varnothing$ detector centers, creating bursts of new particles.

\footnotetext{
${ }^{1}$ Some of the antiprotons from the Accumulator are stored in the Antiproton Recycler (AR) inside the MI tunnel and prepared for Tevatron's next store, which improves luminosity.
} 
The Tevatron not only advances collision energy but also luminosity delivery. The Tevatron's instantaneous luminosity

$$
\mathcal{L}_{i n s}=10^{-6} \frac{f B N_{p} N_{\bar{p}}\left(6 \beta_{r} \gamma_{r}\right)}{2 \pi \beta^{*}\left(\epsilon_{p}+\epsilon_{\bar{p}}\right)} H\left(\frac{\sigma_{l}}{\beta^{*}}\right)
$$

is in units of $10^{31} \mathrm{~cm}^{-2} \mathrm{~s}^{-1}$, where the constants

$f=47.7 \mathrm{kHz}$ stands for revolution frequency;

$B=36$ for number of bunches;

$\beta_{r} \gamma_{r}=1045$ where subscript $r$ stands for relativistic condition;

$\beta^{*}=35 \mathrm{~cm}$ for the $\beta$ function at the interaction point;

$H=0.60-0.75$ for the so-called hourglass factor;

\section{Collider Run II Peak Luminosity}

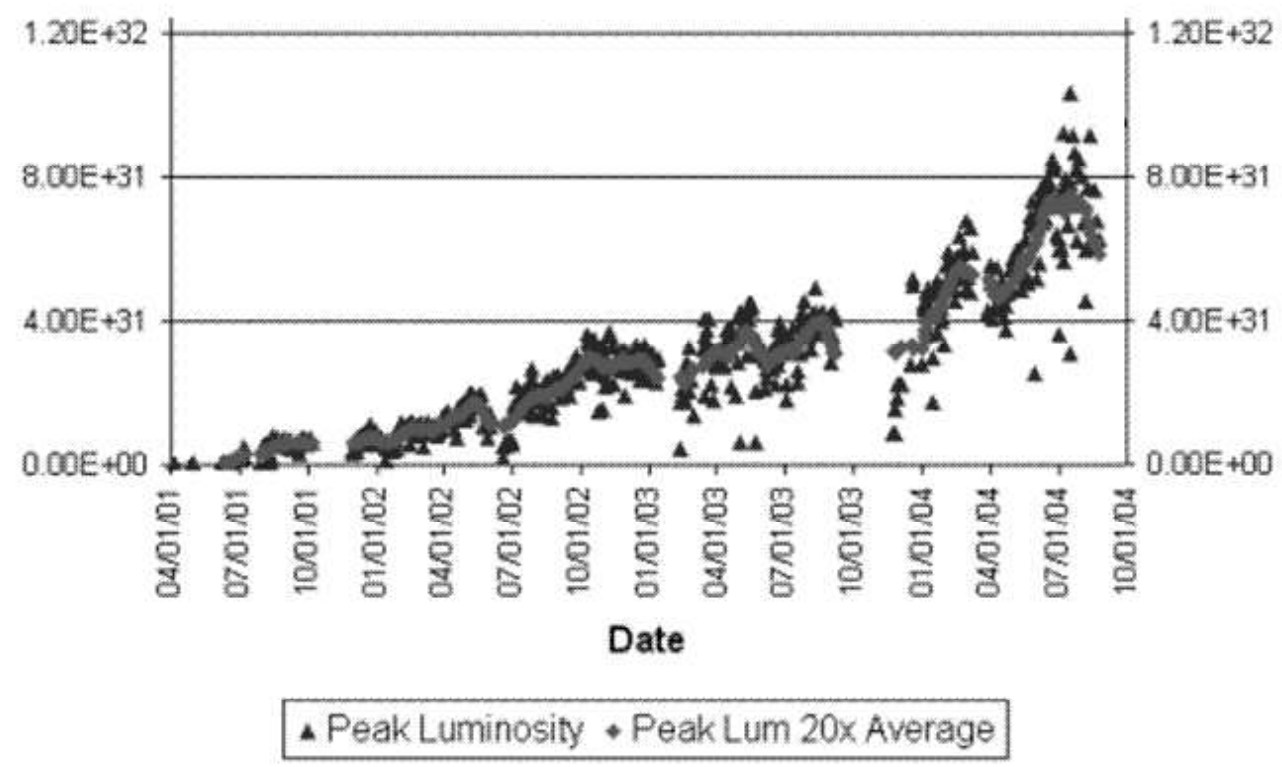

Figure 3.3: History of the Tevatron's luminosity delivery. 
and the variables

$N_{p}, N_{\bar{p}}$ are for bunch intensities in units of $10^{9}$;

$\epsilon_{p}, \epsilon_{\bar{p}}$ are for transverse emittances in units of $\pi$-mm-mrad;

$\sigma_{l}$ is for bunch length in $\mathrm{cm}$.

At the beginning of Run II, the Tevatron's luminosity delivery was ten times below the design goal due to several factors, many of which associated with antiproton production. Over the years, Fermilab has strived to reach the original luminosity design goal successfully, as evidenced in Figure 3.3; in fact, it has surpassed the original design goal and further advanced its reach in particle physics.

\subsection{Overview of the Run II(a) Upgraded CDF Detector}

The CDF II detector [31] is a general purpose cylindrical detector, centered at the B0 collision point along the beamline of Tevatron. It was upgraded from Run I to meet Tevatron Run II luminosity challenges and CDF Run II physics goals. It consists of precision charged particle trackers, fast projective calorimeters and finely grained muon detectors. A longitudinal view of the CDF II detector layout is shown in Figure 3.4. Detector readouts, data acquisition and trigger designs must cope with the 396 ns bunch crossing interval for optimal performance. Detector components must withstand years of aging and radiation hardness through $\mathrm{fb}^{-1} \mathrm{~s}$ luminosity delivery. For general purposes in advancing physics, the detector should measure and trigger on as many event elements as possible for offline reconstruction of light and heavy flavor $^{2}$ jets, leptons and neutrino-caused missing energy etc. These goals defined the

\footnotetext{
${ }^{2}$ Light flavors include the $u, d, c$ and $s$ quarks. Heavy flavors include the $t$ and $b$ quarks.
} 


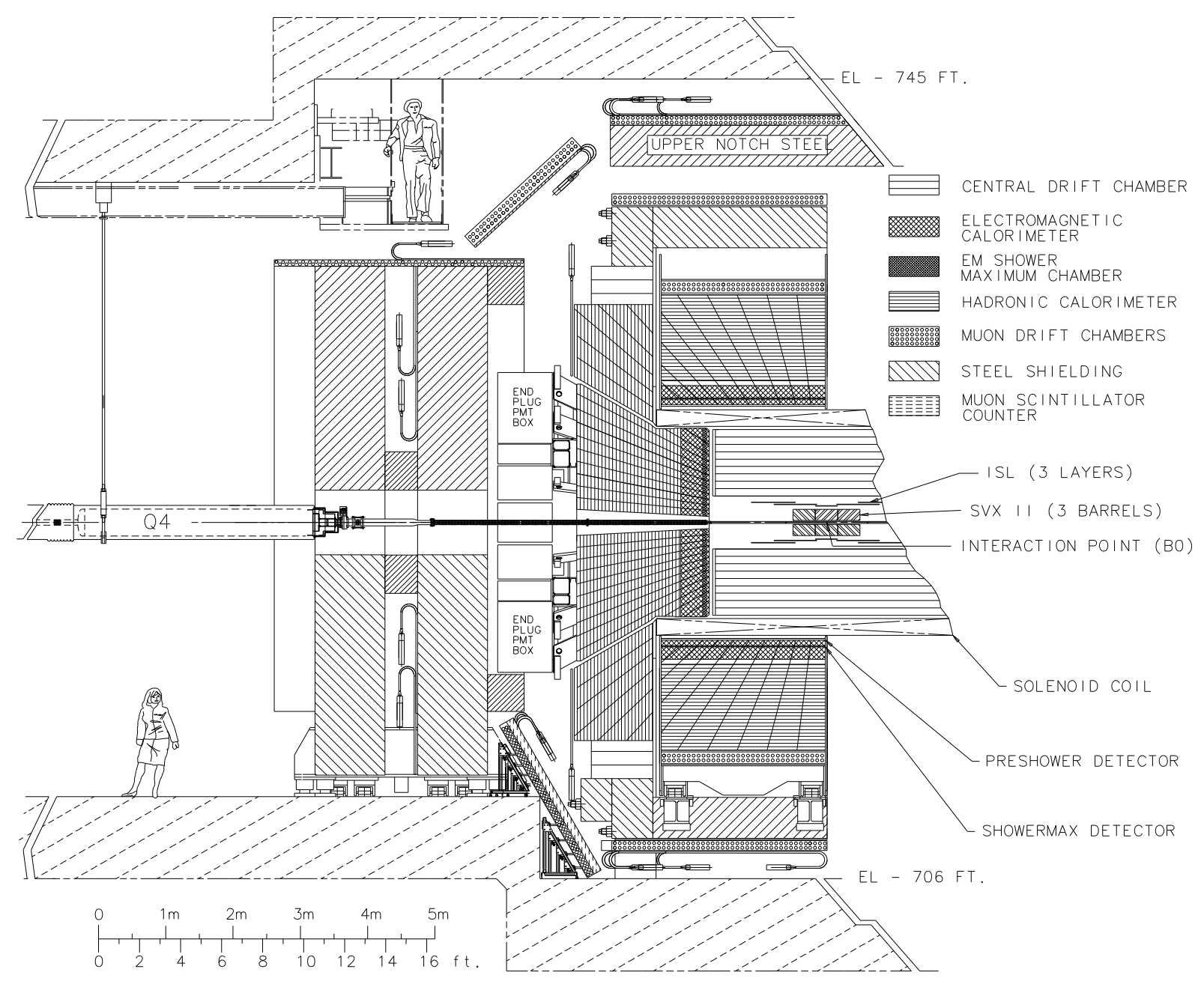

Figure 3.4: Longitudinal view of half of the CDF Run II(a) detector. 
design plans of CDF II detector. The overall data taking efficiency of the CDF II detector is $85-90 \%$.

\subsection{Trackers}

The CDF II tracking system is integrated with silicon vertex detectors closest to the interaction point and a large drift chamber in the radially immediate outer neighborhood. The precious silicon vertex detectors provide excellent spatial resolution in an extensive rapidity region for tracking and secondary vertex reconstruction. The large outer tracker is not as expensive and it provides reliably good position and momentum resolution for tracking in the central detector region of rapidity $|\eta|<1$.

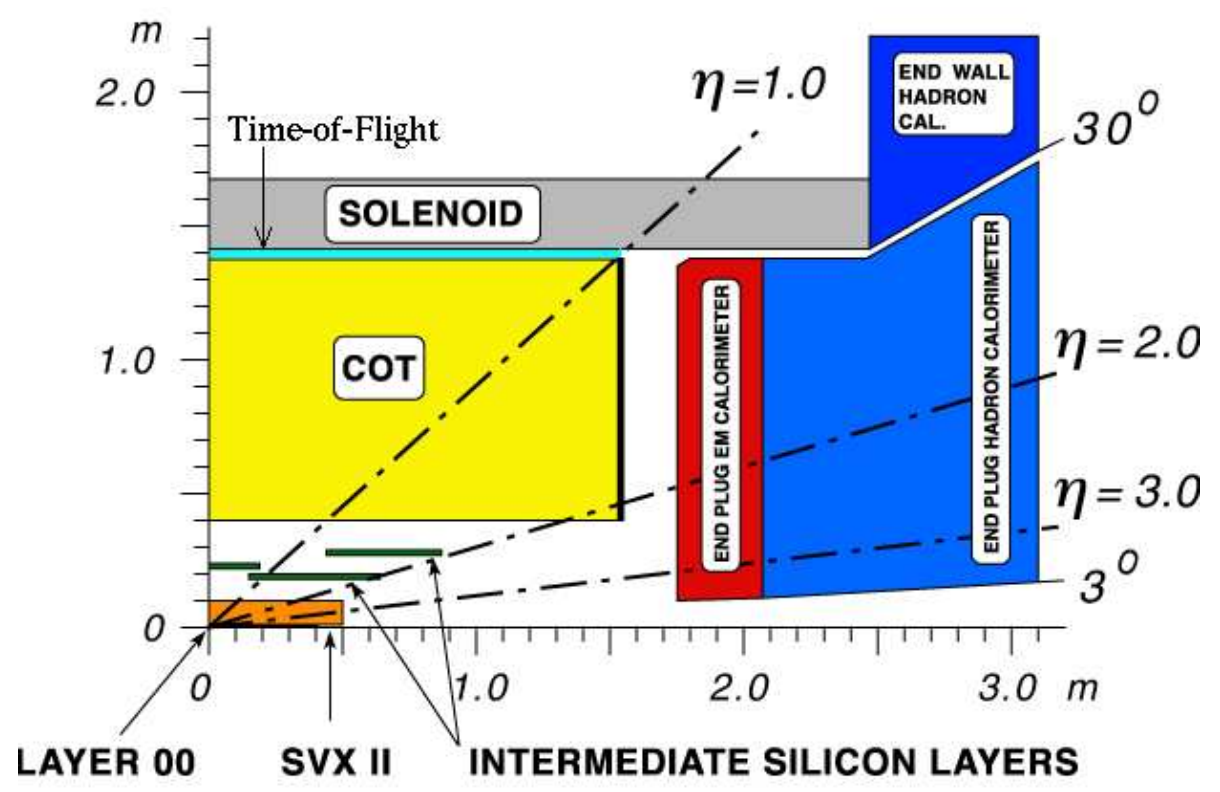

Figure 3.5: Longitudinal view of a quarter of the CDF II tracking system. 


\subsubsection{Superconducting Solenoid}

A superconducting solenoid is located between the trackers and the calorimeters to provide a magnetic field for the measurement of the charged particle's trajectory, momentum and electric charge sign. With an aluminum-stablized niobium-titanium (Al-stablized $\mathrm{NbTi}$ ) conductor inside, the solenoid provides an axial magnetic field of 1.4 Tesla over a useful tracking volume of $r=140 \mathrm{~cm}$ and $z= \pm 175 \mathrm{~cm}$ with an operating current of 4650 Amperes.

\subsubsection{Central Outer Tracker}

The central outer tracker (COT) is a large open cell drift chamber that performs charged particle tracking in the central outer space with inner and outer radii $r_{i}=$ $40 \mathrm{~cm}$ and $r_{o}=137 \mathrm{~cm}$ and length $z= \pm 155 \mathrm{~cm}$. It is filled with argon-ethanecarbontetrafluoride $\left(\mathrm{Ar}-\mathrm{Et}-\mathrm{CF}_{4}\right)$ gas mixture in a ratio of 50:35:15, with drift velocity $100 \mu \mathrm{m} / \mathrm{ns}$. The maximum drift distance is $0.88 \mathrm{~cm}$ to ensure a maximum drift time of less than the $132 \mathrm{~ns}$ bunch-crossing interval for Run II(b). The drift field of $2.5 \mathrm{kV} / \mathrm{cm}$ results in a Lorentz and cell tilt angle of $35^{\circ}$.

The COT consists of 8 superlayers (SL) of $144+48 \times(\mathrm{SL}-1)+24 \times(\mathrm{SL}=1)$ cells, with a layout as shown in Figure 3.6, aligned with a stereo angle of $\pm 3^{\circ}$ between neighboring superlayers. Each cell is closed mechanically and electrostatically by shaper panels, cathode panels, east and west end plates, as shown in Figure 3.6. Along the center line of each cell 12 sense wires alternate with anode wires, all in gold-plated tungsten (Au-plated W). A picture of the COT is shown in Figure 3.7.

A drift chamber basically capitalizes the fact that time delay between the passage of a charged particle through the chamber and the creation of a pulse at the anode is 


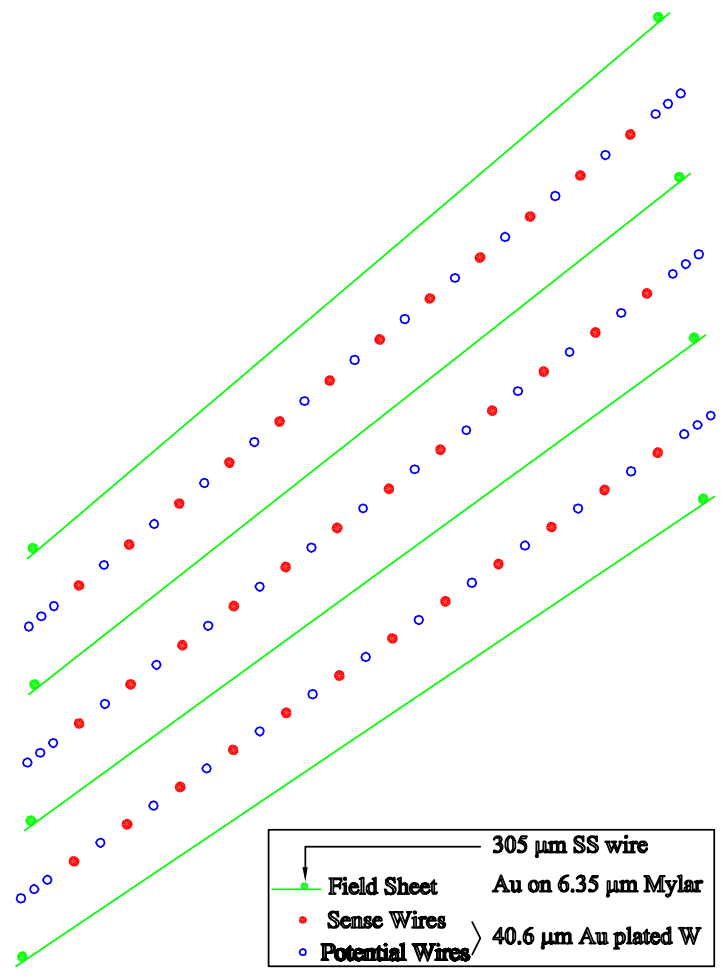

Figure 3.6: Nominal layout of three COT cells.

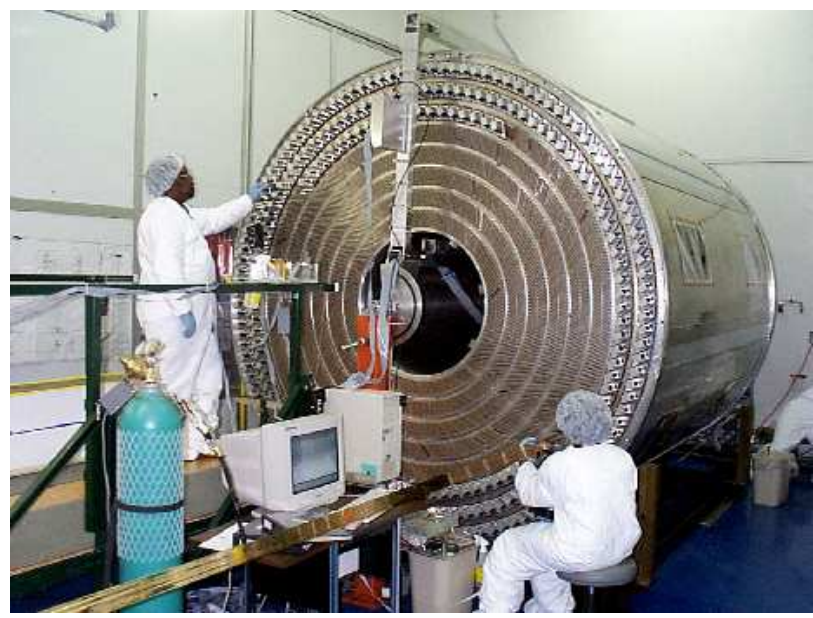

Figure 3.7: Photograph of the COT, taken in LBL. 
proportional to the distance between the particle trajectory and the anode wire. The COT bases its reference time on the bunch crossing counter.

The COT has 30,240 channels in total. Its readout goes through pulse amplification, shaping and discrimination (ASD) to a time-to-digital converter (TDC) event buffer for trigger. A prototype COT cell has a hit resolution of $110 \mu \mathrm{m}$. The COT tracking efficiency is about $95 \%$.

\subsubsection{Silicon Vertex Detectors}

The silicon vertex detectors have proved to be good for $b$ tagging and hence important to analyses related to heavy quarks, light Higgs bosons or any topics that have $b$ jets in the final state.

Silicon microstrip detectors are essentially arrays of solid-state ionization chambers, where electrons and counterpart holes in the electric field separate and collect at electrodes, giving an analog signal proportional to the energy loss of an incident particle. Silicon is costly but it can be manufactured compactly and squeezed into the
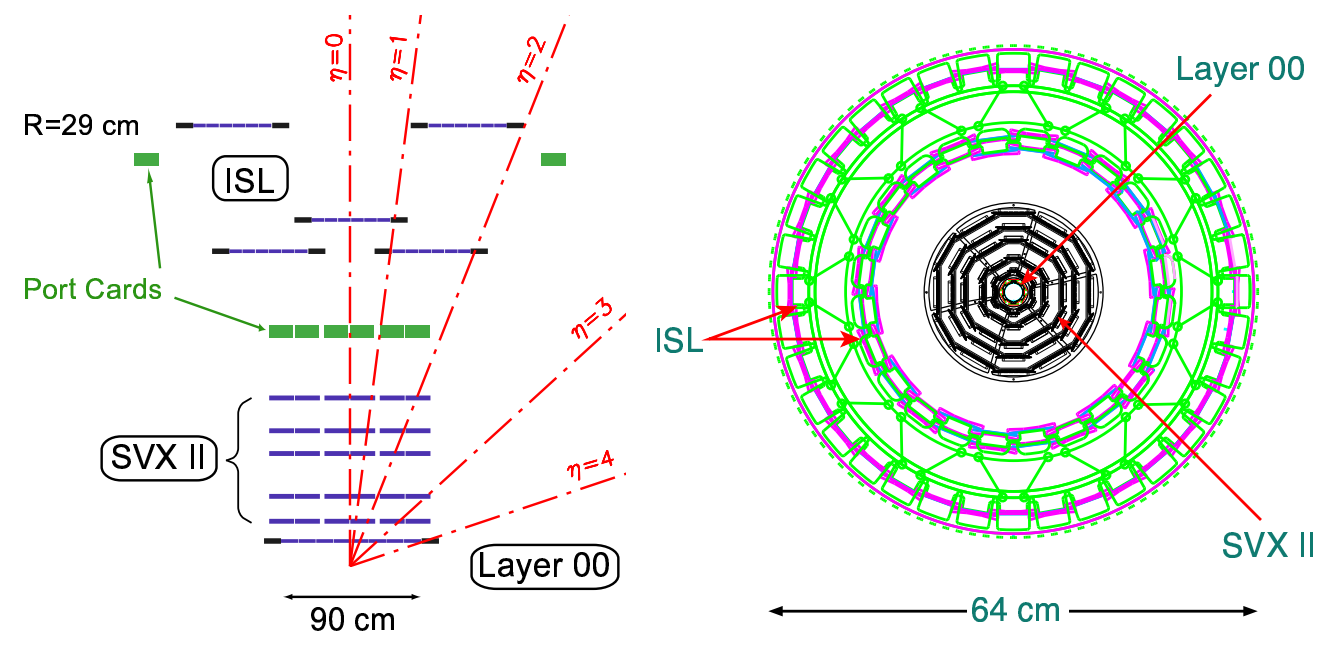

Figure 3.8: Schematic $r z$ (left) and $r \phi$ (right) views of CDF II silicon vertex detectors. 
detector center to achieve a spatial resolution of $10 \mu \mathrm{m}$ for precision reconstruction of impact parameters and recognition of secondary vertices from heavy flavor weak decays.

\subsubsection{Run II(a) Upgraded Silicon Vertex Detector}

The silicon vertex detector upgraded for Run II (SVX II) consists of 3 barrels of 5 double-sided silicon microstrip sensor layers. The inner radius of the innermost layer is $r_{i}=2.4 \mathrm{~cm}$ while the outer radius of the outermost layer is $r_{o}=10.6 \mathrm{~cm}$. The total length is $z= \pm 48 \mathrm{~cm}$. The SVX II covers approximately a $2.5 \sigma$ luminous region and has an almost complete geometric acceptance for $b$-tagging and tracking within a $15 \mathrm{~cm}$ interaction region.

There are 211,968 $r \phi$ channels and 193,536 rz channels, totaling to 405,504 readout channels. A 42-cell analog pipeline per channel stores data during the formation of Level 1 (L1) triggers per 396 ns bunch-crossing interval. The pipeline is buffered and dual-ported to simultaneously output data for digitalization and read in new analog data in order to limit deadtime and to reach a L1 trigger accept rate of order $50 \mathrm{kHz}$.

\subsubsection{Silicon Layer00}

The 0th silicon layer (L00) is a single-sided silicon microstrip sensor layer, mounted close to the beampipe and meant to enhance the SVX II impact parameter resolution and pattern recognition. With inner and outer radii $r_{i}=1.35 \mathrm{~cm}$ and $r_{o}=1.62 \mathrm{~cm}$ and the same length $z= \pm 48 \mathrm{~cm}$ as the SVX II but closer to the beamline, the L00 has a superb rapidity coverage of $|\eta|<4$. 


\subsubsection{Intermediate Silicon Layers}

The intermediate silicon layers (ISL) enhances silicon performance at the most needed places. It contains a cylindrical silicon layer at radius $r_{0}=22 \mathrm{~cm}$ for the central region of $|\eta|<1$ and two at $r_{1}=20 \mathrm{~cm}$ and $r_{2}=28 \mathrm{~cm}$ for the region of incomplete COT track coverage $1<|\eta|<2$, as shown in Figure 3.8, and extends to length $z= \pm 87 \mathrm{~cm}$.

The sensor and readout design for the ISL is basically the same as for the SVX II but the ISL is mechanically easier to support and, for cost saving at large radii, the ISL has longer and wider microstrips and hence fewer readout channels and coarser DAQ segmentation. The SVX II and the ISL are double-sided to enhance pattern recognition and 3-dimensional vertex reconstruction with an impact parameter resolution of $\sigma_{r \phi}<$ $30 \mu \mathrm{m}$ and $\sigma_{z}<60 \mu \mathrm{m}$ for high- $p_{t}$ central tracks. The ISL has a lower hit occupancy, which is an advantage for pattern recognition.

The silicon detectors are radiation-hard enough to survive a luminosity delivery of $3 \mathrm{fb}^{-1}$ as estimated prior to the start of Run II(a). In fact, there is no need for Run II(b) replacement.

The SVX II and ISL tracking performances are maximized over the region of $|\eta|<2$ by complete SVX II track coverage. The SVX II and the L00, of which the

\begin{tabular}{|l|cc|c|}
\hline \hline & SVX II & + ISL & COT \\
\hline \hline$\delta d_{0}(\mu \mathrm{m})$ & 50 & 15 & 600 \\
\hline$\delta p_{t} / p_{t}{ }^{2}(\%)$ & 7. & .4 & .3 \\
\hline \hline
\end{tabular}

Table 3.1: Comparison of the SVX II, SVX II + ISL and COT impact parameter $d_{0}$ and transverse momentum $p_{t}$ resolutions. 
tracking performances fall off in the forward regions due to partial coverage, are the only trackers for $2<|\eta|<3.4$.

A comparison of silicon vs. COT resolutions is shown in Table 3.1. The SVX II together with the ISL has tracking efficiency about $95 \%$ for $|\eta|<2$. The ISL raises single and double $b$-tagging efficiency from $0.467 \pm 0.011$ and $0.087 \pm 0.006$ to $0.601 \pm 0.010$ and $0.151 \pm 0.008$.

\subsection{Calorimeters}

The CDF II calorimetry system measures energy by sampling scintillator response with central electromagnetic and hadronic calorimeters located radially outside the solenoid, forward electromagnetic and hadronic calorimeters located longitudinally outside the COT and a hadronic calorimeter between central and forward rapidities to make continuous electromagnetic and hadronic calorimetric cylinders up to $|\eta|<3.64$, enclosing the interaction point around the beamline with few gaps between subsystems. The calorimeter geometric coverages are available in Figure 3.4.

The calorimeters, electromagnetic in front and hadronic in back, are segmented by towers matching between subsystems for ease of offline reconstruction. The basic structure of a calorimetric tower is energy sampling scintillator layers interleaved with energy absorbing metal layers, readout by a photomultiplier tube (PMT). Sampling measurement necessitates calibration using reference sources.

The electromagnetic calorimeters each embed a scintillator strip chamber at shower maximum depth to measure position and shower profile with higher resolution. The electromagnetic calorimeters are also each with a preshower radiation detector 
attached in front for systematic uncertainty reduction.

\subsubsection{Central Calorimeters}

The central calorimeters are inherited from Run I and are segmented into towers of $\Delta \eta=0.11$ and $\Delta \phi=15^{\circ}$. A wedge of the central electromagnetic calorimeter is shown in Figure 3.9.

\subsubsection{Central Electromagnetic Calorimeter}

The central electromagnetic calorimeter (CEM) occupies the volume of $r_{i}=$ $173 \mathrm{~cm}, r_{o}=207 \mathrm{~cm}$ and $z= \pm 250 \mathrm{~cm}$. It has a rapidity reach of $|\eta|<1.1$.

The CEM consists of 31 scintillator sampler layers interleaved with 30 lead $(\mathrm{Pb})$ absorber layers. Each scintillator layer is $5 \mathrm{~mm}$ thick, made of radiation hard SCSN38 with a wavelength shifter Y7-PMMA. The scintillator signal is readout by two Hamamatsu R580 PMT's per tower on each side. The calibration system of the CEM is complemented by LED and xenon (Xe) flashers and cesium $\left({ }^{137} \mathrm{Cs}\right)$ sources. Each lead absorber layer is $3 \mathrm{~mm}$ thick. The total depth of the CEM is 18 radiation lengths $\left(X_{0}\right)$ and 1 pion interaction length $\left(\lambda_{0}\right)$. The CEM has an energy resolution ${ }^{3}$ of $\delta E / E \approx 13.5 \% / \sqrt{E_{t}(\mathrm{GeV})}$.

The central preshower radiation detector (CPR) is located in front of the CEM. It is a wire chamber that uses the solenoid coil and tracking material as a radiator. It greatly enhances photon and soft electron identification, which improves $b$-tagging

\footnotetext{
${ }^{3} E_{t}=E \sin \theta$, where $E_{t}$ is the transverse component of total energy $E$ and $\theta$ is the polar angle of either the highest- $E_{t}$ calorimetric tower or the highest- $p_{t}$ COT track pointing to the calorimetric cluster.
} 
and other algorithms that use soft electrons.

The central electromagnetic strip-wire chamber (CES, a.k.a. showermax) is embedded at the shower maximum depth of the CEM, $r=185 \mathrm{~cm}$, consisting of orthogonally laid scintillator strips and Au-plated W wires. The CES greatly enhances photon and electron identification by measuring traversing particle position for later track matching, transverse shower profile for photon and pion separation and pulse

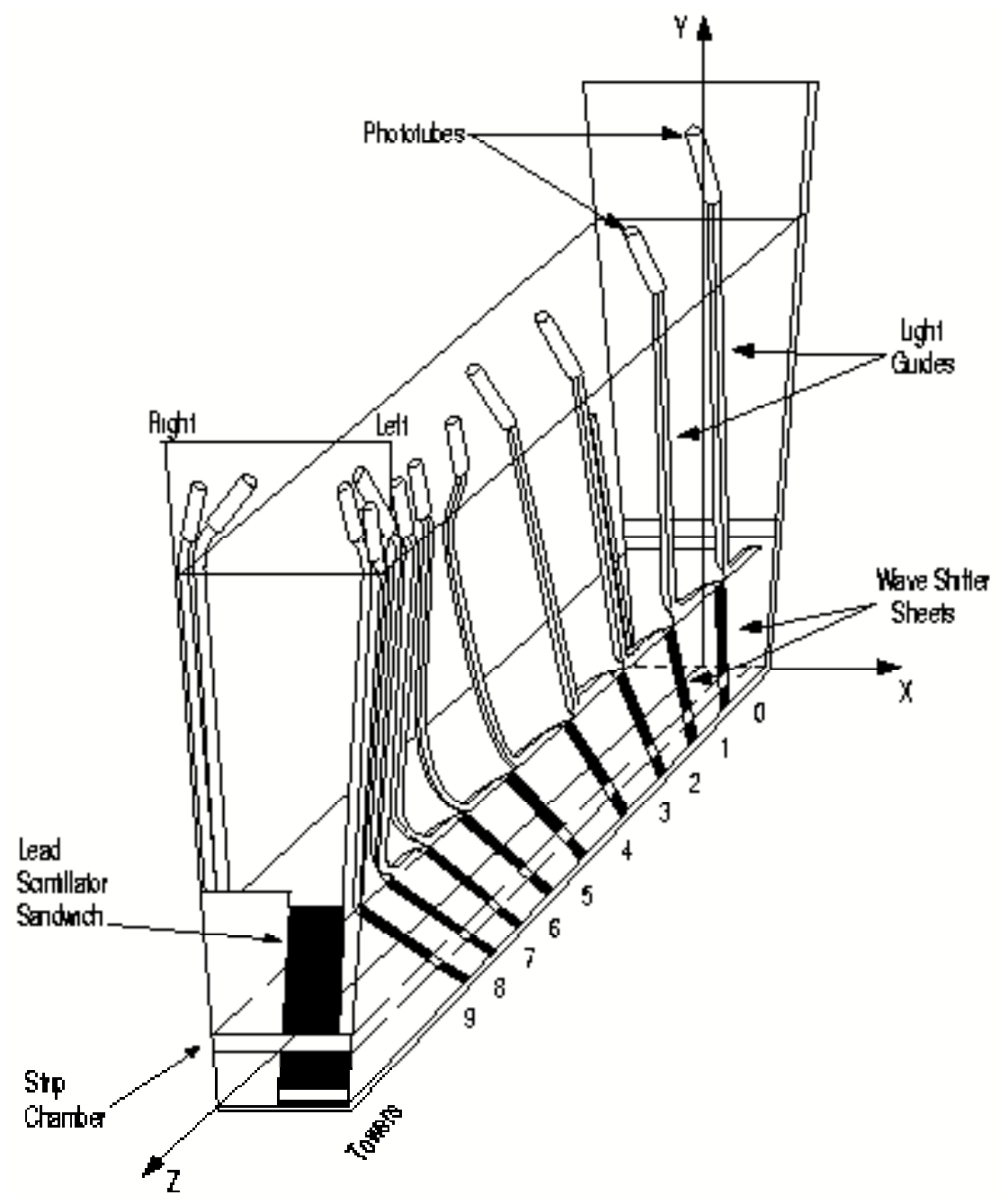

Figure 3.9: Schematic view of a CEM wedge. 
height for electromagnetic shower identification. The CES has a position resolution of $\delta x \simeq 2 \mathrm{~mm}$ for a particle of energy $50 \mathrm{GeV}$ in the CES $\phi z$ plane.

\subsubsection{Central Hadronic Calorimeter}

The central hadronic calorimeter (CHA) occupies a volume of $r_{i}=214 \mathrm{~cm}$, $r_{o}=347 \mathrm{~cm}$ and $z= \pm 250 \mathrm{~cm}$. It has a rapidity reach of $|\eta|<0.9$.

The CHA consists of 32 scintillator sampler layers interleaved with 32 steel (Fe) absorber layers. The scintillator configuration for the CHA is the same as for the CEM except that each layer is $10 \mathrm{~mm}$ rather than $5 \mathrm{~mm}$ thick. The calibration system of the CHA is complemented by LED flasher, strontium $\left({ }^{90} \mathrm{Sr}\right) \beta$ and ${ }^{137} \mathrm{Cs}$ sources. Each steel absorber layer is $25 \mathrm{~mm}$ thick. The total depth of the CHA is $4.7 \lambda_{0}$. The CHA measures jet energy with resolution $\delta E / E \approx 50 \% / \sqrt{E(\mathrm{GeV})}$.

\subsubsection{Wall Hadronic Calorimeter}

The wall hadronic calorimeter (WHA) occupies a volume of $z_{i}= \pm 250 \mathrm{~cm}$, $z_{o}= \pm 327 \mathrm{~cm}, r_{o}=347 \mathrm{~cm}$ and $\left|\eta_{o}\right|=1.32$.

The WHA consists of 15 scintillator sampler layers interleaved with 15 steel (Fe) absorber layers. The scintillator configuration and calibration for the WHA is an extension of that for the CHA. Each steel absorber layer is $50 \mathrm{~mm}$ thick - twice the CHA steel absorber layer thickness to save expense while keeping enough energy resolution since the total energy into the WHA is more than $\sqrt{2}$ the total energy into the CHA for the same amount of transverse energy. The total depth of the WHA is $4.5 \lambda_{0}$. The WHA measures jet energy with resolution $\delta E / E \approx 50 \% / \sqrt{E(\mathrm{GeV})}$. 


\subsubsection{Endplug Calorimeters}

The plug calorimeters are newly built for Run II. The physical and trigger tower segmentations of plug calorimeters per $15^{\circ}$ wedge are shown in Figure 3.10.

\subsubsection{Plug Electromagnetic Calorimeters}

The plug electromagnetic calorimeter (PEM) occupies a volume of $z_{i}= \pm 173 \mathrm{~cm}$, $z_{o}= \pm 123 \mathrm{~cm}, r_{o}=132 \mathrm{~cm}$ and $\left|\eta_{o}\right|=3.64$. It has a rapidity reach of $1.1<|\eta|<3.6$.

The PEM consists of 22 scintillator sampler layers interleaved with 23 leadcompound absorber layers. Each scintillator layer is $4 \mathrm{~mm}$ thick, made of SCSN-38 with Kuraray wavelength shifter and read out by one Hamamatsu H1161 PMT per tower. The PEM calibration system uses a roaming ${ }^{137} \mathrm{Cs}$ source. Each calcium-tin-
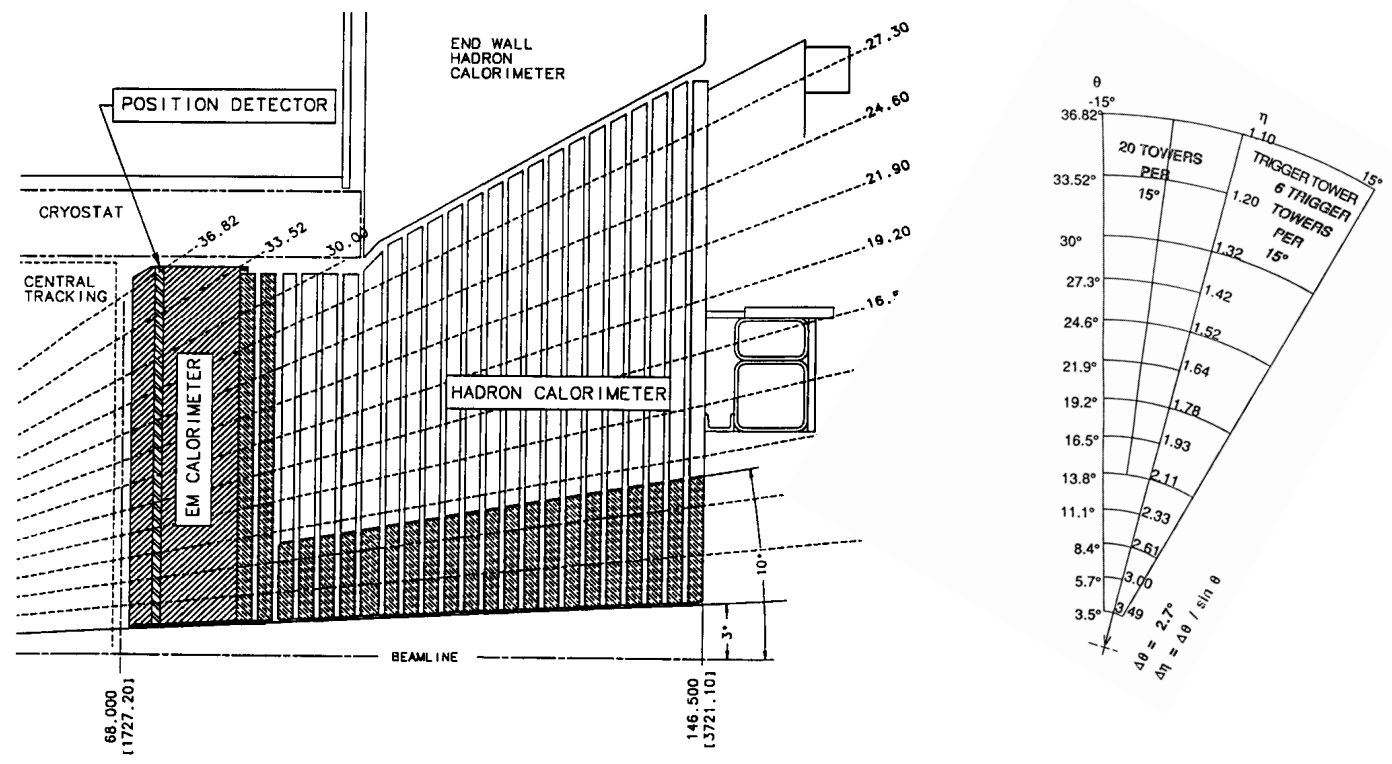

Figure 3.10: Longitudinal view of a plug calorimetric quarter (left). Transverse view of plug calorimetric physical and trigger tower segmentation (right). 
lead (Ca-Sn-Pb) absorber layer is $4.5 \mathrm{~mm}$ thick. The total depth of the PEM is $21 X_{0}$ and $1 \lambda_{0}$. The PEM has an energy resolution of $\delta E / E \approx 16 \% / \sqrt{E(\mathrm{GeV})}$.

The first scintillator layer of the PEM, $10 \mathrm{~mm}$ thick and read out by one multianode photomultiplier tube (MAPMT) per tower, functions as a plug preshower radiation detector (PPR) for enhancing photon and soft electron identification.

The plug electromagnetic strip counters (PES) are embedded at the shower maximum depth of the PEM, $z \sim 6 X_{0}$, and consist of two layers of scintillator strips, called $\mathrm{U}$ and $\mathrm{V}$, aligned with $\pm 22.5^{\circ}$ off-radius in 8 sectors. It measures position and transverse shower profile for an improvement in photon and electron identification. The PES has a position resolution of $\delta x \simeq 1 \mathrm{~mm}$ in the PES $r \phi$ plane.

\subsubsection{Plug Hadronic Calorimeters}

The plug hadronic calorimeter (PHA) occupies a volume of $z_{i}= \pm 123 \mathrm{~cm}$, $z_{o}= \pm 372 \mathrm{~cm}, r_{o}=132 \mathrm{~cm},\left|\eta_{i}\right|=1.32$ and $\left|\eta_{o}\right|=3.64$. It has a rapidity reach of $1.2<|\eta|<3.6$

The PHA consists of 21 scintillator sampler layers interleaved with 21 iron absorber layers. The scintillator configuration and calibration for the PHA is the same as for the PEM except that each layer is $6 \mathrm{~mm}$ rather than $4 \mathrm{~mm}$ thick. Each iron absorber layer is $5 \mathrm{~cm}$ thick. The total depth of the PHA is $7 \lambda_{0}$. The PHA measures jet energy with resolution $\delta E / E \approx 80 \% / \sqrt{E(\mathrm{GeV})}$.

\subsection{Muon Detectors}

The CDF II muon detector system consists of drift tube chambers and scintillator counters, altogether covering a rapidity range of $|\eta|<2$. Muon detection is based on 
the particle's non-interactivity; therefore the muon detectors, being the outermost components, are behind most of the CDF II detector material and many of them are further behind thick steel. The muon detector geometric coverages are available in Figure 3.4.

\subsubsection{Central Muon Detector}

The central muon detector (CMU) is inherited from Run I. The CMU is located right outside the $\mathrm{CHA}$ behind $5.5 \lambda_{0}$ of detector material. It has a rapidity range of $|\eta|<0.68$. It is a barrel with $r_{i}=347 \mathrm{~cm}$ and $r_{o}=369 \mathrm{~cm}$, consisting of 4 drift tube layers sectioned by wedge matching the CHA towers; 3 sections of 4 tubes per layer per $15^{\circ}$ wedge.
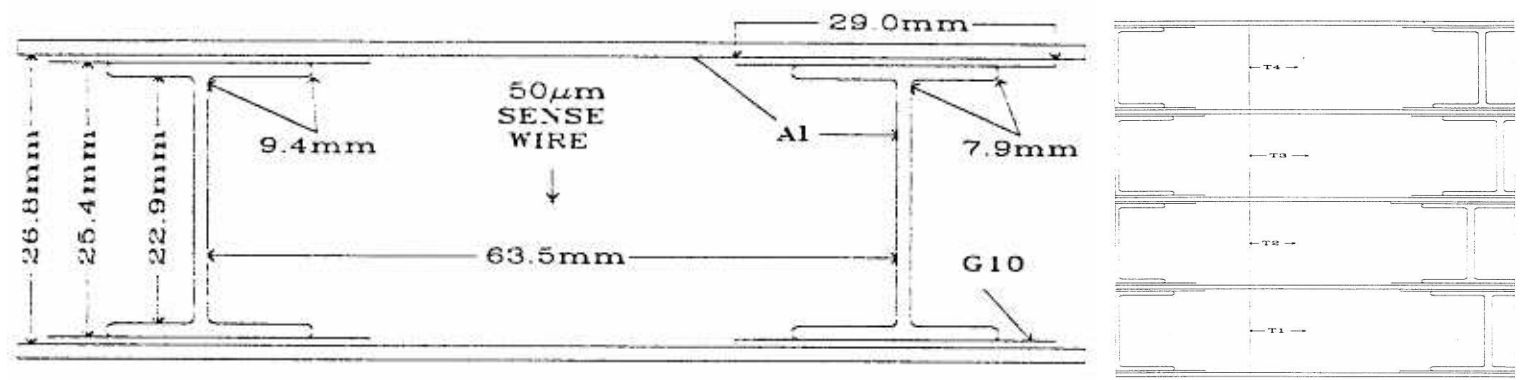

Figure 3.11: Transverse view of a CMU drift tube (left) and of a CMU stack (right).

The CMU drift tube design is shown in Figure 3.11. Each tube contains argonethane-alcohol $\left(\mathrm{Ar}-\mathrm{C}_{2} \mathrm{H}_{6}-\mathrm{C}_{2} \mathrm{H}_{5} \mathrm{OH}\right)$ 50:50:0.7 gas mixture ${ }^{4}$, a steel wire anode center

\footnotetext{
${ }^{4}$ Gas mixture recipes for drift tubes have been empirical. For the CDF II muon detectors, the noble gas argon is chosen for good proportionality, high gain, swift drift, slow diffusion, long lifetime and fast recovery as well as avalanche at low voltage and low cost; the quencher ethane is added to prevent Ar ions from liberating electrons from cathode metals because $\mathrm{C}_{2} \mathrm{H}_{6}$ has more atoms and hence more ways to absorb energy than a single Ar atom. A tiny amount of alcohol is added to
} 
held at voltage $+2325 \mathrm{~V}$ and aluminum cathode strip sidewalls at $-2500 \mathrm{~V}$ when in operation. The CMU was once operated in the limited streamer gas mode; it was changed to the proportional mode after pre-amplifiers were affixed. The maximum drift time is $0.8 \mu \mathrm{s}$ for tube cross-section $6.35 \times 2.68 \mathrm{~cm}^{2}$ and length $226 \mathrm{~cm}$.

The CMU has a transtube multiple scattering resolution of $12 /[p(\mathrm{GeV} / c)] \mathrm{cm}$ and a longitudinal position resolution of $\delta z \approx 10 \mathrm{~cm}$ with the use of charge division between anode wire ends.

The CMU is calibrated using iron $\left({ }^{55} \mathrm{Fe} X\right.$-ray) sources and complemented with timing information from the CHA time-to-digital converter (TDC).

\subsubsection{Central Muon uPgrade}

The central muon upgrade detector (CMP) is located outside the CMU behind $7.8 \lambda_{0}$ of detector material that includes additional $60 \mathrm{~cm}$ thick steel slabs. The CMP contains four layers of rectangularly arrayed drift tubes, as shown in Figure 3.12; the tubes are half-cell staggered. The CMP tube planes are $\pm 3.2 \mathrm{~m}$ long and $\pm 5.2 \mathrm{~m}$ away from the beamline. The CMP has a rapidity extension of $|\eta|<0.68$, the same as the CMU, although due to geometry the actual rapidity coverage varies with azimuth.

The CMP greatly improves central muon identification efficiency because hadronic punch-through is suppressed by the pre-CMP steel slabs and track-stub matching can be doubly confirmed by using both the CMU and the CMP.

The CMP drift tube design is shown in Figure 3.13. Each tube contains Ar$\mathrm{C}_{2} \mathrm{H}_{6}-\mathrm{C}_{2} \mathrm{H}_{5} \mathrm{OH}$ 50:50:0.7 at 1.13 atm at $-5^{\circ} \mathrm{C}$ gas mixture, in which a Au-plated W wire anode center held at $+5.6 \mathrm{kV}$ and aluminum cathode strips on the bottom and 
the top at $+3 \mathrm{kV}$ when in operation. The CMP gas operation mode is proportional. The maximum drift time is $1.4 \mu \mathrm{s}$ for tube cross-section $15 \times 2.5 \mathrm{~cm}^{2}$ and length $640 \mathrm{~cm}$. The CMP is calibrated using ${ }^{55} \mathrm{Fe} X$-ray sources. The CMP has a transtube multiple scattering resolution of $15 /[p(\mathrm{GeV} / c)] \mathrm{cm}$.

A layer of NE-114 scintillators (CSP) is mounted onto the outside surface of the CMP to provide timing information for the CMP. The CSP mounting is half-cell staggered to improve the CMP position resolution. A CSP scintillator is only half of a CMP tube long to improve its own timing resolution. The CSP greatly improves muon identification efficiency because it allows for comparison between stub registry time and bunch-crossing time. The CSP is calibrated using ${ }^{137} \mathrm{Cs}$ sources and has a timing resolution of 1-2 ns.

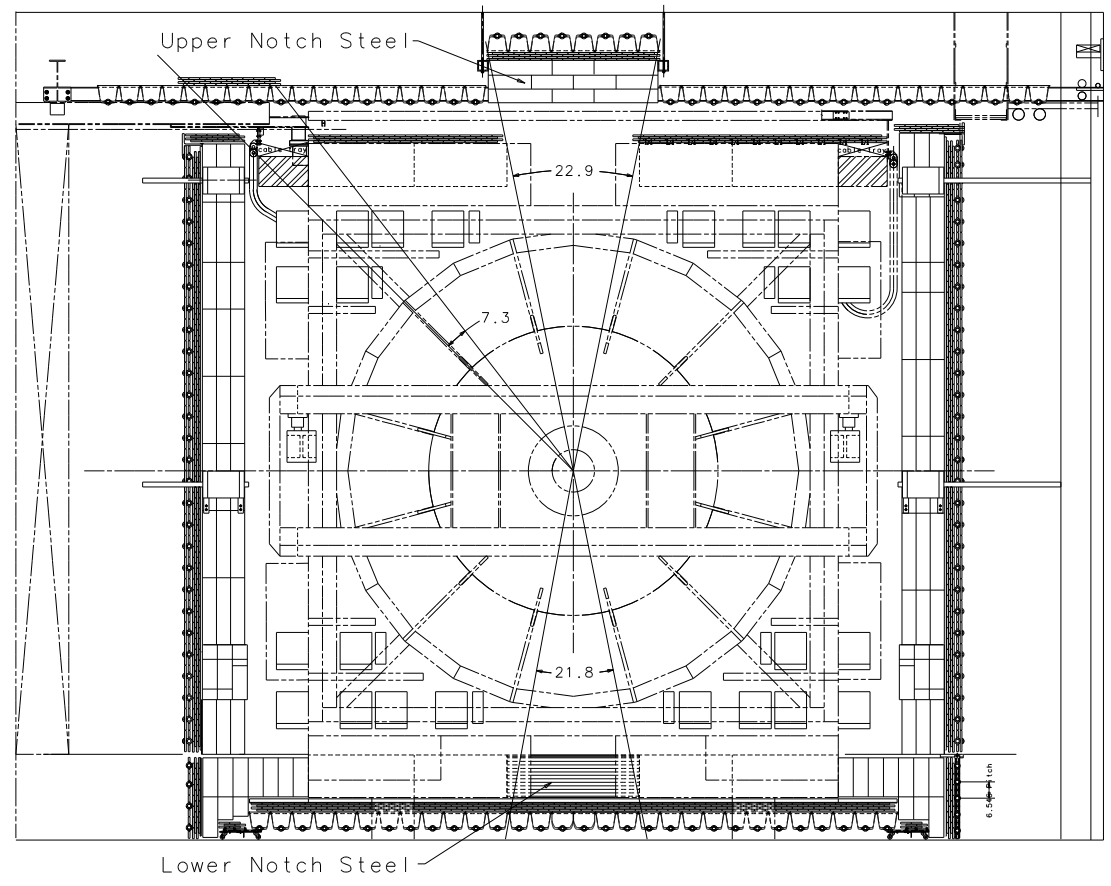

Figure 3.12: Transverse view of the CMP geometry. 


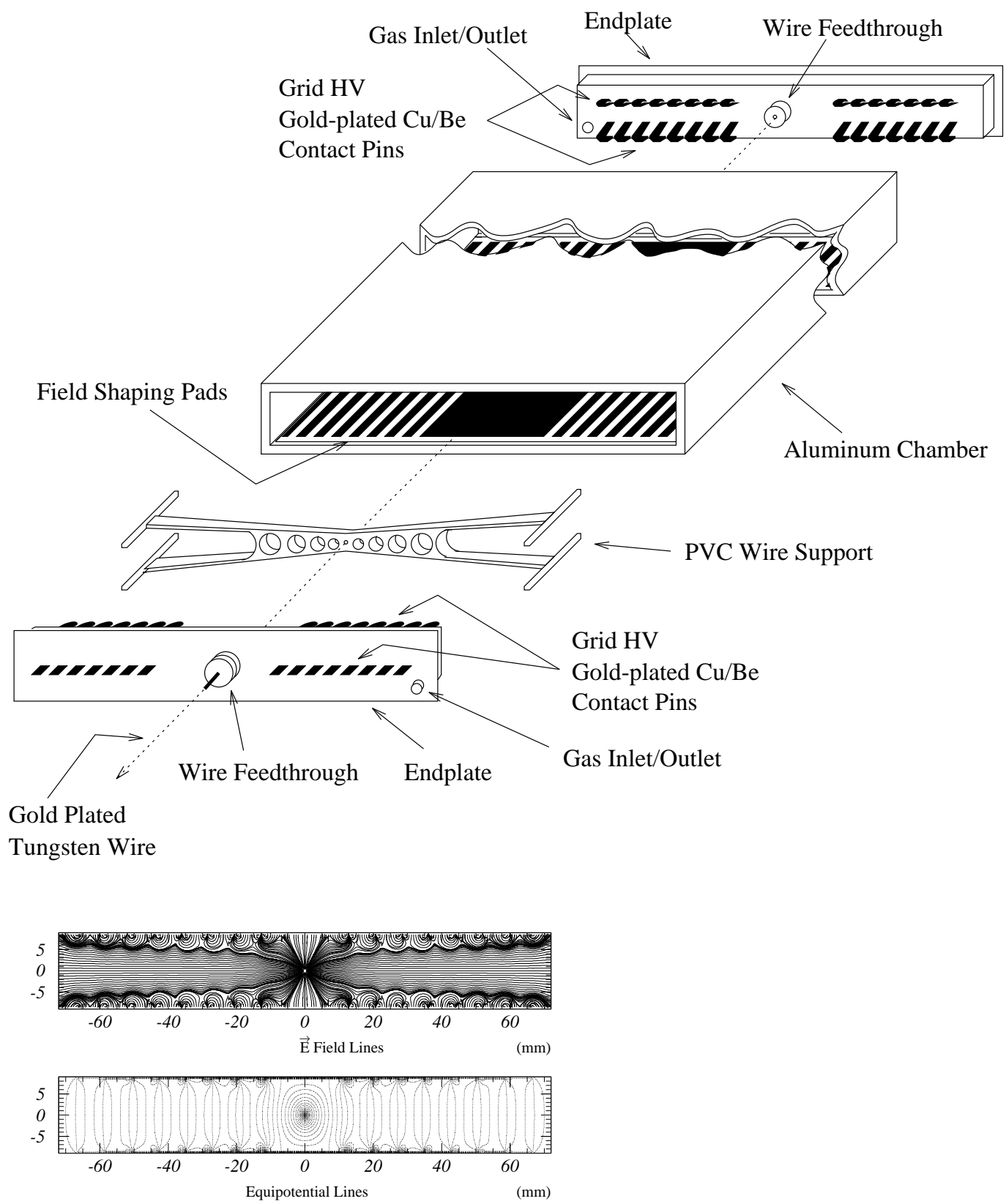

Figure 3.13: Mechanical design of a CMP/CMX/IMU drift tube (upper) and the electric field lines and equal-potential lines inside a CMP/CMX/IMU drift tube (lower). 

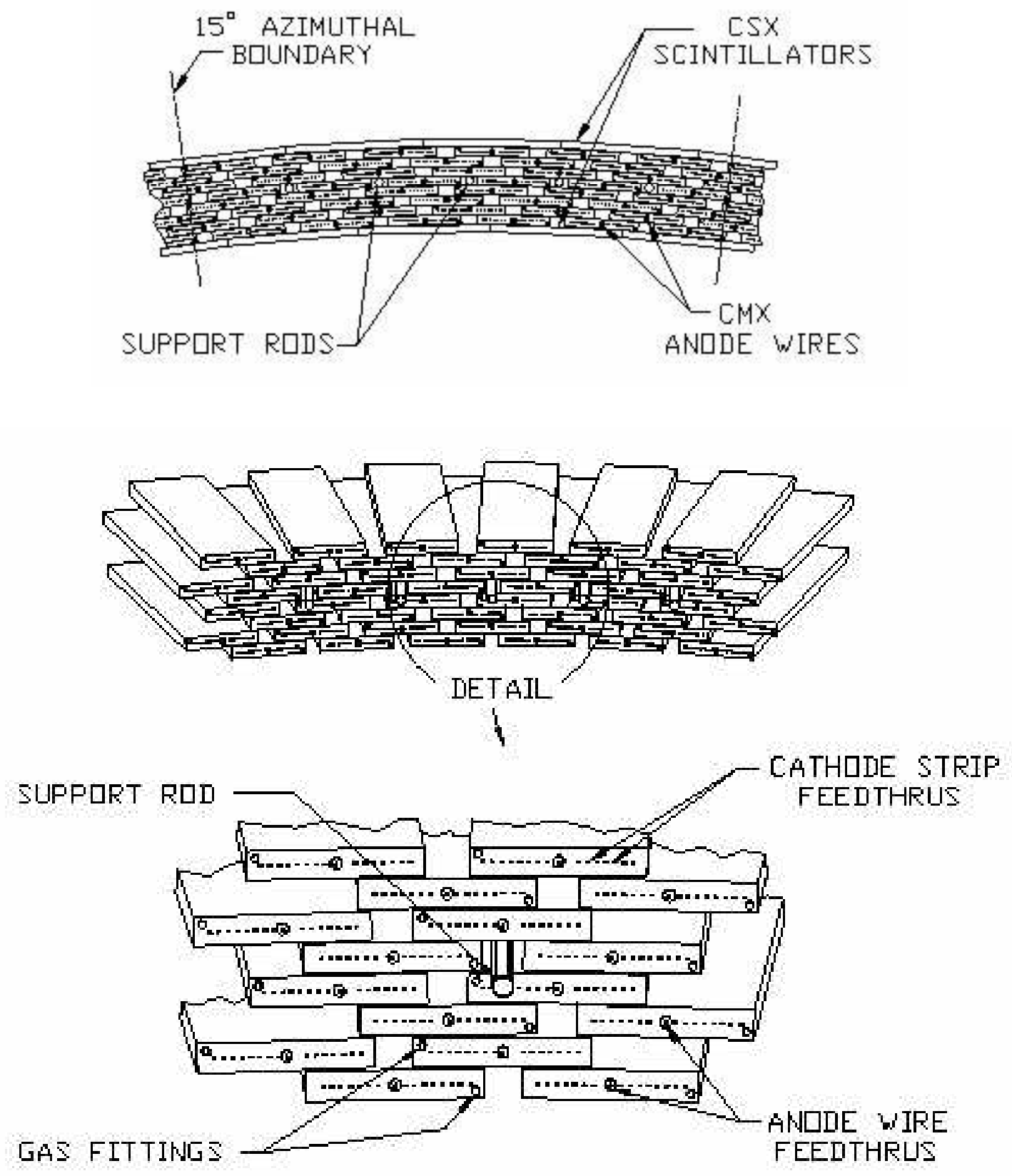

Figure 3.14: Transverse view of the CMX and CSX stacks. 


\subsubsection{Central Muon eXtension}

The central muon extension detector (CMX) consists of conical sections facing toward the interaction point behind $6.2 \lambda_{0}$ of detector material, extending the central muon detectors by rapidity $0.65<|\eta|<1.0$ excluding the east top $30^{\circ}$ in azimuth. The CMX contains two folds of 4 layers of half-cell staggered rectangular drift tubes, using the same tube design and calibration as the CMP but with a tube length of $180 \mathrm{~cm}$, with larger overlap at higher absolute rapidity, as shown in Figure 3.14.

The CMX has a transtube multiple scattering resolution of $13 /[p(\mathrm{GeV} / c)] \mathrm{cm}$ and a longitudinal position resolution of $\delta z \approx 14 \mathrm{~cm}$ with the aid of drift tube overlap.

Two layers of scintillators (CSX) are mounted with one layer to the upper surface and the other to the lower surface of the CMX to provide timing information for the CMX, using the same scintillator material, mounting scheme and calibration design as the CSP but with a trapezoidal tube width of $30-40 \mathrm{~cm}$ to fully cover the conical CMX chamber planes.

\subsubsection{Intermediate Muon Detector}

The intermediate muon detector (IMU) is upgraded from Run I for advanced performance in Run II. The IMU is built around the outside surface of shielding steel toroids behind $6.2-20 \lambda_{0}$ of detector material depending on rapidity. The IMU consists of two $\frac{3}{4}$-barrels $^{5}$ with radius $r=385 \mathrm{~cm}$, inner length $z_{i}= \pm 4.7 \mathrm{~m}$ and outer length $z_{o}= \pm 8.1 \mathrm{~m}$, extending the CDF II geometric muon acceptance by $1.0<|\eta|<1.5$ in rapidity.

\footnotetext{
${ }^{5}$ The incomplete IMU barrels meet the floor.
} 
The IMU contains four layers of half-cell staggered drift tubes (BMU), using a similar tube design and calibration to the CMP but with a tube width of $8.4 \mathrm{~cm}$ and a tube length of $363 \mathrm{~cm}$. The maximum drift time is $0.8 \mu \mathrm{s}$.

The BMU has a transtube multiple scattering resolution of $13-25 /[p(\mathrm{GeV} / c)] \mathrm{cm}$ and a longitudinal position resolution of $\delta z \approx 16.5 \mathrm{~cm}$ with every two anode wires jumpered together, as shown in Figure 3.15, to facilitate the measurement of signal transmission time difference between the readout ends, which is proportional to the incident particle's $z$ distance from the jumpered end.

Two (front and rear) layers of scintillators (BSU-F and BSU-R) are mounted on the outside surface of the BMU, using a similar scintillator design, mounting and calibration to the CSP but with a tube length of $180 \mathrm{~cm}$ so that the BSU-F and BSU-R scintillators together cover one BMU drift tube in length. A layer of scintillator (TSU) is mounted onto the rear surface of inner toroids, spanning $1.3<|\eta|<2$ in rapidity. The timing system of the BMU includes the BSU, the TSU and the WHA/PHA TDC.

\section{Ad]acent wlreg ganged}

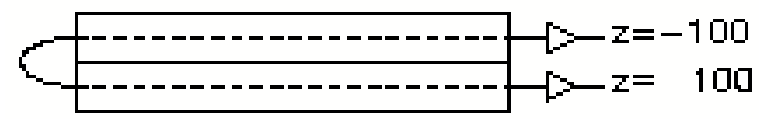

Figure 3.15: Illustration of two jumpered BMU drift tubes. Within a stack, the BMU 0th and 2nd or 1st and 3rd layer's anode wires are jumpered together at one end and each connected to a pre-amplifier for readout at the other end. 

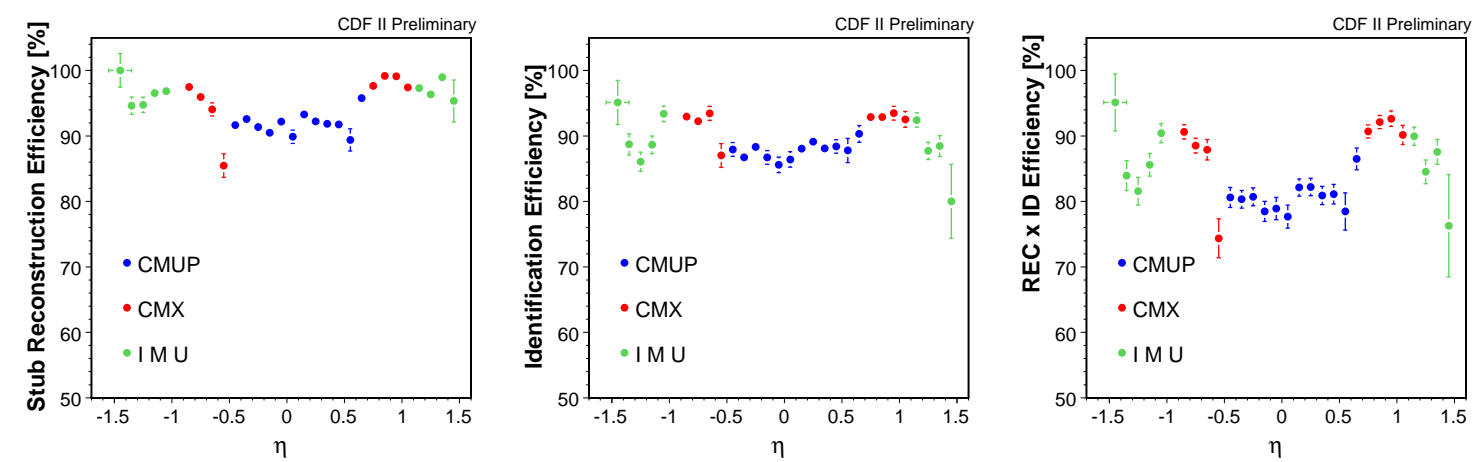

Figure 3.16: Muon reconstruction and identification efficiencies as a function of detector rapidity in 2006 .

\subsection{Data Acquisition}

The CDF Run II front-end electronics (FE) and data acquisition (DAQ) systems meet the challenges from the Tevatron on luminosity and bunch-crossing interval with high rate data transmission and deadtimeless DAQ.

To accommodate the 396 ns bunch-crossing interval and a $5.5 \mu$ s decision time at the first trigger level (L1), all front-end electronics are fully pipelined with on-board buffers for every 42 crossings. Data from the central outer tracker, the calorimeters and the muon detectors are sent to L1, which determines if the bunch-crossing is interesting enough for more transfers, including from the silicon vertex detectors, to the second trigger level (L2). There are four L2 buffers for a $20 \mu$ s decision time. L2 determines if the event is interesting enough for readout - if so, data are collected in DAQ buffers and then transferred through a network switch to a CPU node of the third trigger level (L3), where the event is completely assembled, analyzed and, if accepted, written out to permanent storage tape. With an accept rate of $40 \mathrm{kHz}$ at L1 and $300 \mathrm{~Hz}$ at L2, the DAQ system has less than $10 \%$ deadtime. The event writing 


\section{Dataflow of CDF "Deadtimeless" Trigger and DAQ}

\section{L1 Storage}

Pipeline:

42 Clock

Cycles Deep

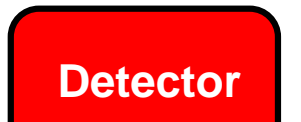

7.6 $\mathrm{MHz}$ Crossing rate

132 ns clock cycle

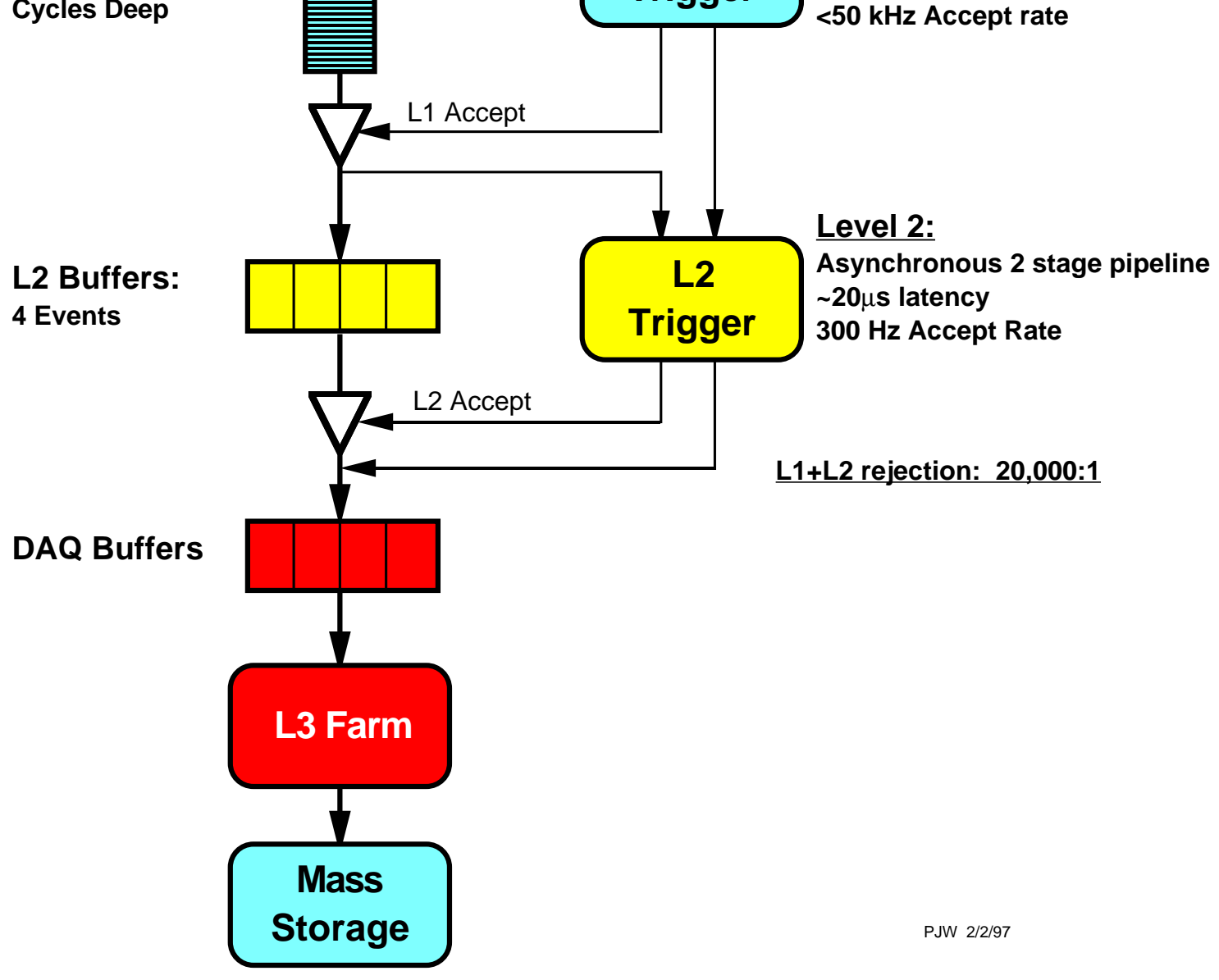

Figure 3.17: Block diagram of the deadtimeless CDF II DAQ system. 
rate of L3 is $80 \mathrm{~Hz}$ on average. A chart illustrating the CDF II DAQ system is shown in Figure 3.17.

\subsection{Trigger Overview}

Trigger plays an important role in a hadron collider experiment like CDF because the collision rate is much higher than the feasible data storage rate. The role of trigger is to efficiently extract interesting physics events out of $\mathrm{MHz}$ minimum bias events.

The CDF II trigger system is in a three-level architecture with each level performing sufficient rate reduction to allow the next level to process with minimum deadtime. L1 uses custom designed hardware to find physics objects based on a subset of detector information and determines based on simple counting of objects in terms of numbers and energies of electron, muon and jet candidates and missing transverse energy. L2 uses custom hardware to do limited event reconstruction on programmable electronic boards and to decide based on reconstruction results. L3 uses full detector information to reconstruct events in a processor farm.

Significant additions on trigger from Run I to Run II include:

1. COT track finder (XFT) at L1, which enables track matching to an electromagnetic calorimetric cluster or a muon stub as well as the track alone being used in trigger;

2. silicon vertex tracker (SVT) at L2, which allows triggering on secondary vertices and the possibility of track matching to a forward muon stub.

A block diagram of the CDF Run II trigger system is shown in Figure 3.18. 


\section{RUN II TRIGGER SYSTEM}

Detector Elements

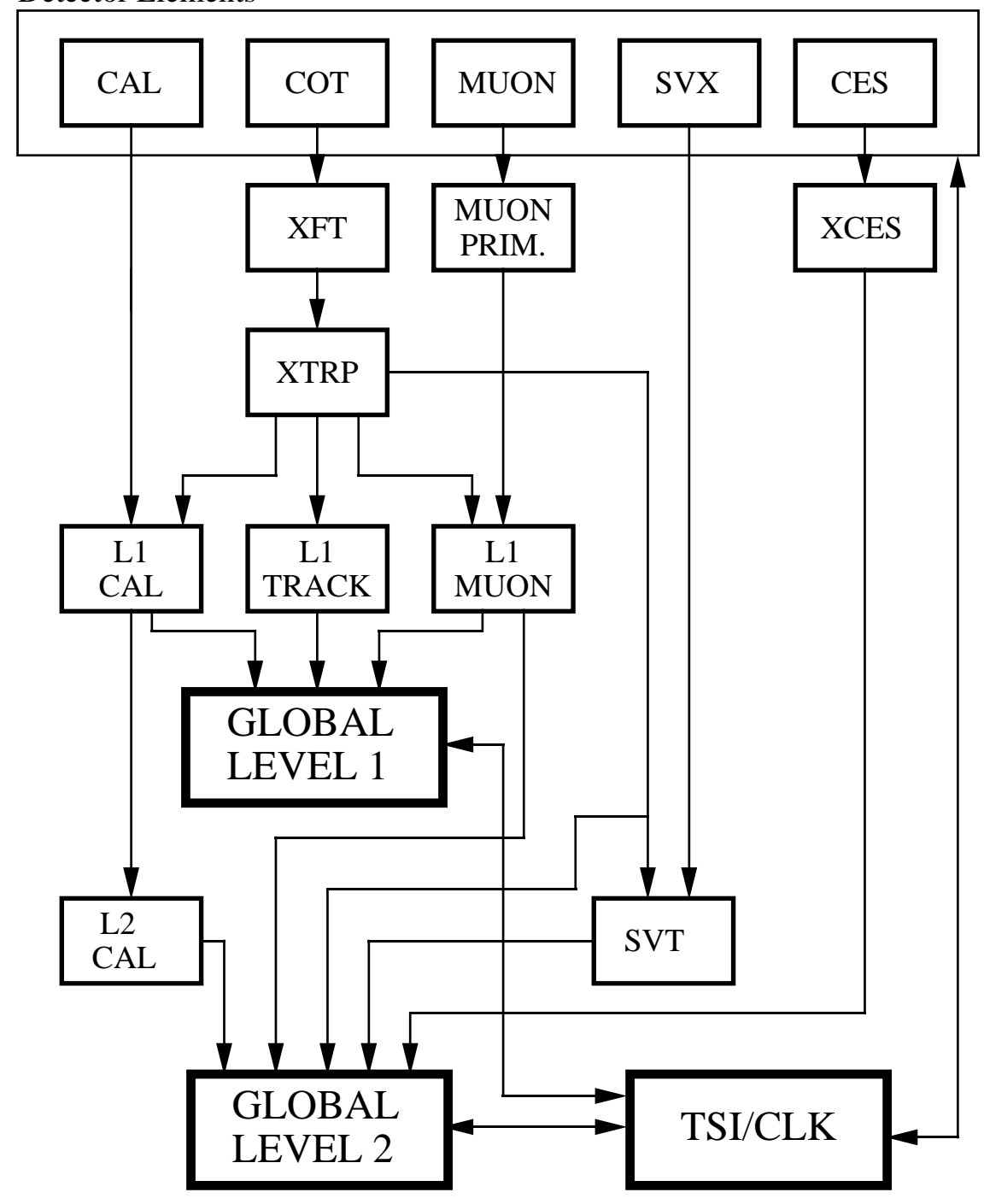

Figure 3.18: Block diagram of the CDF II trigger system. The trigger system interface and clock (TSI/CLK) synchronizes trigger and DAQ. 


\section{Chapter 4}

\section{Data Description}

Data used in this analysis were collected by the CDF II Detector from March 2002 to September 2004 and processed in the cdfsoft2 5.3.1 environment.

\subsection{Triggers}

Data used in this analysis came from four triggers that are described below.

\subsubsection{ELECTRON_CENTRAL_18}

The high- $E_{t}$ central electron trigger consists of the following requirements from L1 to L3:

L1 CEM8_PT8 requires a trigger tower in the central calorimetric region with an electromagnetic transverse energy deposit of $E_{t e m}>8 \mathrm{GeV}$ and a ratio of hadronic to electromagnetic energy deposit of $E_{h a d} / E_{e m}<0.125$; it also requires a track reconstructed by the eXtremely Fast Tracker (XFT) for the COT with 10 hits in 3 layers or 11 hits in 4 layers and with a transverse momentum of $p_{t}>8.34 \mathrm{GeV} / c$.

L2 CEM16_PT8 requires a calorimetric cluster with $|\eta|<1.317$, an electromagnetic 
transverse energy deposit in the seed tower $E_{t}$ em seed $>8 \mathrm{GeV}$, in shoulder towers $E_{t \text { em } \text { shoulder }}>7.5 \mathrm{GeV}$ and total $E_{t}$ em $>16 \mathrm{GeV}, E_{\text {had }} / E_{e m}<0.125$ and an XFT track with 10 hits in 3 layers or 11 hits in 4 layers and with $p_{t}>8 \mathrm{GeV} / c$.

L3 ELECTRON_CENTRAL_18 requires an offline central electromagnetic calorimetric cluster with $E_{t}>18 \mathrm{GeV}$ and $E_{\text {had }} / E_{e m}<0.125$ and a COT track with $p_{t}>9 \mathrm{GeV}$. Since May 2003 it has additionally required lateral shower profile $L_{s h r}<0.4$, matching between the CES cluster and the COT track $|\Delta z|<8 \mathrm{~cm}$,

\begin{tabular}{|c|c|}
\hline \hline period & start-end run number \\
\hline \hline$\# 1$ & $141544-147869$ \\
\hline$\# 2$ & $148153-152629$ \\
\hline$\# 3$ & $152630-156487$ \\
\hline$\# 4$ & $159603-163527$ \\
\hline$\# 5$ & $163955-167715$ \\
\hline$\# 6$ & $167717-179099$ \\
\hline$\# 7$ & $179105-184060$ \\
\hline$\# 8$ & $184062-186598$ \\
\hline \hline
\end{tabular}

Table 4.1: Run periods, on which the ELECTRON_CENTRAL_18 trigger efficiencies depend.

\begin{tabular}{|c|c|c|c|c|}
\hline$\overline{\text { L\# trigger }}$ & \multicolumn{4}{|c|}{$\bar{L}$ L\# trigger efficiency } \\
\hline $\begin{array}{l}\text { L1_CEM8 } \\
\text { L2_CEM16 } \\
\text { L3_CEM18 }\end{array}$ & \multicolumn{4}{|c|}{$\begin{array}{c}\epsilon_{\text {trg }}>0.9999 \\
0.9988-3319 e^{-0.5512 E_{t}} \\
1-2.784 e^{-1.749\left(E_{t}-17.86\right)}\end{array}$} \\
\hline run period & $\# 1$ & \#2 & $\# 3$ & $\# 4$ \\
\hline L1-3 tracking & $0.9628(25)$ & $0.9783(13)$ & $0.9619(12)$ & $0.9615(12)$ \\
\hline run period & $\# 5$ & $\# 6$ & $\# 7$ & $\# 8$ \\
\hline L1-3 tracking & $0.9579(12)$ & $0.9606(11)$ & $0.9653(16)$ & $0.9785(07)$ \\
\hline
\end{tabular}

Table 4.2: ELECTRON_CENTRAL_18 trigger efficiencies, not requiring the silicon vertex detector in good condition. 


\begin{tabular}{|c|c|c|c|c|}
\hline L\# trigger & \multicolumn{4}{|c|}{ L\# trigger efficiency } \\
\hline $\begin{array}{l}\text { L1_CEM8 } \\
\text { L2_CEM16 } \\
\text { L3_CEM18 }\end{array}$ & \multicolumn{4}{|c|}{$\begin{array}{c}\epsilon_{\text {trg }}>0.9999 \\
0.9987-3386 e^{-0.5491 E_{t}} \\
1-2.784 e^{-1.749\left(E_{t}-17.86\right)}\end{array}$} \\
\hline run period & $\# 1$ & $\# 2$ & \#3 & $\# 4$ \\
\hline L1-3 tracking & $0.9651(35)$ & $0.9782(14)$ & $0.9606(13)$ & $0.9612(12)$ \\
\hline run period & $\# 5$ & $\# 6$ & $\# 7$ & \#8 \\
\hline L1-3 tracking & $0.9579(12)$ & $0.9611(11)$ & $0.9646(17)$ & $0.9785(07)$ \\
\hline
\end{tabular}

Table 4.3: ELECTRON_CENTRAL_18 trigger efficiencies, requiring the silicon vertex detector in good condition.

using the vertex $z$ position instead of the interaction point for the transverse component and three instead of two towers for $E_{\text {had }} / E_{\text {em }}$ calculations.

The dataset ID in the CDF II data file catalogue (DFC ID) for the ELECTRON_CENTRAL_18 data used by this analysis is bhel0d and the study of corresponding trigger efficiencies is documented in [32]. The ELECTRON_CENTRAL_18 trigger efficiencies depend on run period due to the changes in XFT trigger requirements. The run periods are listed in Table 4.1. The efficiencies, separated by the requirements on the silicon vertex detector condition, trigger level and run period as needed for analysis coding, are listed in Table 4.2 and Table 4.3.

\subsubsection{MET_PEM}

The plug electron plus the missing transverse energy trigger consists of the following requirements from L1 to L3:

L1 EM8_MET15 requires a calorimetric trigger tower with $E_{t}$ em $>8 \mathrm{GeV}$ and $E_{\text {had }} / E_{\text {em }}<0.125$; it also requires raw missing transverse energy $\mathbb{E}_{t}>15 \mathrm{GeV}$ and the sum of transverse energy deposits in the calorimeters $\Sigma E_{t}>1 \mathrm{GeV}$. 
L2 PEM20_L1_EM8_MET15 requires a calorimetric cluster in $1.1<|\eta|<3.6$ with $E_{t \text { em seed }}>8 \mathrm{GeV}, E_{t \text { em } \text { shoulder }}>7.5 \mathrm{GeV}$ and $E_{t \text { em }}>20 \mathrm{GeV}$ and $E_{\text {had }} / E_{\text {em }}<0.125$.

L3 PEM20_MET15 requires an offline plug electromagnetic cluster with $E_{t}>20 \mathrm{GeV}$ and $E_{\text {had }} / E_{e m}<0.125$ and $\mathbb{E}_{t}>15 \mathrm{GeV}$. Since May 2003 it has additionally required the use of the vertex $z$ position instead of the interaction point for transverse component calculations.

The DFC ID for the MET_PEM data used by this analysis is bpel0d and the study of corresponding trigger efficiencies is documented in [33]. The MET_PEM trigger efficiency over the analyzed run periods is $\epsilon_{\mathrm{MET} \_ \text {PEM }}=0.919 \pm 0.004$.

\subsubsection{MUON_CMUP18}

The high- $p_{t}$ CMUP muon trigger consists of the following requirements from L1 to L3:

L1 CMUP6_PT4 requires a stub in the CMU with $p_{t}>6 \mathrm{GeV} / c$ as measured by the $\mathrm{CMU}$ and an XFT track pointing to the $\mathrm{CMU}$ with $p_{t}>4.09 \mathrm{GeV} / c$ as measured by the COT. It also requires a hit in the CMP and the CSP.

L2 TRK8_L1_CMUP6_PT4 requires a CMUP L1-muon, a 4-layer XFT track with $p_{t}>8.34 \mathrm{GeV} / c$ and a minimum ionizing hit in the calorimeters since run 152950.

L3 MUON_CMUP_18 requires a CMUP primitive muon with $p_{t}>18 \mathrm{GeV}$ and distances between the stub and the COT track extrapolated to the muon detector plane of $\Delta x_{\mathrm{CMU}}<10 \mathrm{~cm}$ and $\Delta x_{\mathrm{CMP}}<20 \mathrm{~cm}$. 
The DFC ID for the MUON_CMUP18 data used by this analysis is bhmu0d and the study of corresponding trigger efficiencies is documented in [34]. The MUON_ CMUP18 trigger efficiency over the analyzed run periods is $\epsilon_{\mathrm{CMUP}}=0.908 \pm 0.005$.

\subsubsection{MUON_CMX18}

The high- $p_{t}$ CMX muon trigger consists of the following requirements from L1 to L3:

L1 CMX6_PT8_CSX requires a stub in CMX with $p_{t}>6 \mathrm{GeV} / c$ as measured by the CMX and an XFT track pointing to the CMX with $p_{t}>8.34 \mathrm{GeV} / c$ as measured by the COT. It also requires a hit in the CSX.

L2 CMX6_PT10 requires a CMX L1-muon, a 4-layer XFT track with $p_{t}>10.1 \mathrm{GeV} / c$ and a minimum ionizing hit in the calorimeters since run 181013.

L3 MUON_CMX18 requires a CMX primitive muon with $p_{t}>18 \mathrm{GeV}$ and a distance between the stub and the COT track extrapolated to the muon detector plane of $\Delta x_{\mathrm{CMX}}<10 \mathrm{~cm}$.

The DFC ID for the MUON_CMX18 data used by this analysis is bhmu0d and the study of corresponding trigger efficiencies is documented in [34]. The MUON_ CMX18 trigger efficiency over the analyzed run periods is $\epsilon_{\mathrm{CMX}}=0.965 \pm 0.004$.

\subsection{Good Run Lists}

The detector, as an assembly of numerous complicated devices, inevitably suffers from occasional malfunctioning since hardware might fail due to age or fluctuation of temperature, humidity or gas composition, software might crash due to temperature 
fluctuation, imperfect logic design or electronic instability and also human operators could err in data taking and processing. As a result, data quality varies with time.

The quality of this analysis was ensured with the exclusive use of data from good runs, which were marked per subdetector by data-taking shiftcrew and detector maintenance experts online and offline and enlisted by the Data Quality Monitering (DQM) group.

The good run lists, depending on dilepton type (described in Chapter 5) of the event, were applied in the following way:

1. by default all the detector components were required to be in good condition for analyzable data events. However,

2. a good silicon vertex detector condition was not required for events containing no plug electron;

\begin{tabular}{|c|c|c|}
\hline $\begin{array}{l}\text { Dilepton } \\
\text { Event } \\
\text { Type(s) }\end{array}$ & $\begin{array}{l}\text { Good } \\
\text { Run } \\
\text { List }\end{array}$ & $\begin{array}{l}\text { Offline } \mathcal{L}_{\text {int }}\left(\mathrm{pb}^{-1}\right) \\
+1.9 \% \text { Correction } \\
\text { and } \pm 5.9 \% \text { Errors }\end{array}$ \\
\hline TCE-TCE & 1001 & $374 \pm 22$ \\
\hline PHX-\{TCE, PHX $\}$ & 1101 & $343 \pm 21$ \\
\hline TCE-\{CMUP, CMU, CMP, CMIO $\}$ & 1031 & $366 \pm 22$ \\
\hline TCE-CMX & 1021 & $333 \pm 20$ \\
\hline PHX-\{CMUP, CMU, CMP, CMIO $\}$ & 1131 & $337 \pm 20$ \\
\hline PHX-CMX & 1121 & $310 \pm 19$ \\
\hline CMUP-\{CMUP, CMU, CMP, CMIO $\}$ & 0031 & $378 \pm 23$ \\
\hline CMX-\{CMUP, CMX, CMU, CMP, CMIO $\}$ & 0011 & $339 \pm 20$ \\
\hline
\end{tabular}

Table 4.4: Dilepton event types, good run lists (in the CDF internal 4-digit code) and corresponding corrected integrated luminosities. 
3. a good muon detector condition was not required for events containing no muon;

4. a good $\mathrm{CMU}$ and $\mathrm{CMP}$ detector condition was not required for events containing no CMUP, CMU-only and CMP-only muon;

5. a good CMX detector condition was not required for events containing no CMX muon.

The applied good run lists per dilepton type are listed in Table 4.4.

\subsection{Integrated Luminosities}

The integrated luminosity of each good run list was calculated in the following way:

1. raw integrated luminosity of each run in the good run list was retrieved from the CDF II database;

2. total raw integrated luminosity of each good run list was calculated by summing up the raw integrated luminosities of each run in the good run list;

3. the following corrections were applied to raw luminosities [35]:

(a) $+1.9 \%$ correction for the historical extrapolation of inelastic $p \bar{p}$ cross-section from Run I to Run II in the luminosity calculation at CDF;

(b) $\pm 5.9 \%$ errors due to the luminosity measurement of cherenkov luminosity counter (CLC).

The corrected integrated luminosities per good run list are listed in Table 4.4. 


\subsubsection{Equivalent Integrated Luminosity}

An equivalent integrated luminosity for the analysis

$$
\mathcal{L}_{\text {int }}=360 \pm 22 \mathrm{pb}^{-1}
$$

was obtained by

1. weighing each dilepton type dependent integrated luminosity with the relative acceptance;

2. summing up the weights. 


\section{Chapter 5}

\section{Particle Identification}

The basic elements that constitute an event, such as leptons, jets and missing transverse energy, must be reconstructed with pieces of information on position, time, momentum or energy provided by single or multiple detector subsystems and identified in order to apply event selection cuts with them.

\section{$5.1 \quad$ Leptons}

Leptons include electrons, muons and tau leptons. Experimentally, electrons and muons are easier to reconstruct than tau leptons because electrons and muons live long enough to be directly detected while tau leptons are heavier and shorterlived and hence decays before they can be directly detected. Electrons and muons can be reconstructed with smaller uncertainties than tau leptons since tau leptons can only be reconstructed from its daughters. Reconstructed electrons and muons, but not tau leptons, were used in this analysis.

Lepton reconstruction (REC) and identification (ID) efficiencies are inevitably different between data and Monte Carlo (MC). Lepton REC and ID data/MC scale factors are a consequence of the impossibility for $\mathrm{MC}$ to model everything in data 
perfectly.

\subsubsection{Electrons}

The clustering algorithms, which are basically electron and photon reconstruction, for CDF II electromagnetic objects are well documented in [36].

An electron of energy $E$ showers with a signature profile and deposits most of its energy in the electromagnetic calorimeter. It also interacts with the silicon and COT material and leaves a track so that its momentum $p$ can be measured by the trackers. Basic electron identification requires low $E_{h a d} / E_{e m}, E / p$ close to unity, an electron-like lateral shower profile and the matching of the electron track to a calorimetric cluster.

Electron identification is implemented for both the central and forward detector regions, although the terminologies in use may be different since the calorimeters and the rapidity-matching trackers are different. Central electron identification is more reliable than forward at CDF II because of better COT track coverage ${ }^{1}$ and a lower level of physics background.

\subsubsection{Tight Central Electrons}

A tight central electron (TCE) was selected with the following cuts:

1. there must be a cluster in the $\mathrm{CEM}^{2}$, of which the seed tower is neither tower 7 nor tower 9 ;

2. transverse energy, $E_{t}>10 \mathrm{GeV}$;

\footnotetext{
${ }^{1}$ The COT track coverage is complete in the central region and partial in the forward region.

${ }^{2}$ The rapidity range of central electrons is restricted by the CEM to $\left|\eta_{\text {det }}\right|<1.1$.
} 
3. ratio of hadronic to electromagnetic calorimetric energy deposit in the cluster, $E_{\text {had }} / E_{\text {em }}<0.055+0.00045 E$

4. transverse momentum of the highest- $p_{t}$ COT track pointing to the cluster, $p_{t}>$ $5 \mathrm{GeV}$, as measured using track curvature in COT due to the solenoid's magnetic field; $p_{t}>10 \mathrm{GeV}$ was further required if the candidate would be the highest- $E_{t}$ lepton after all the leptons were identified;

5. ratio of transverse energy of the electromagnetic calorimetric cluster to transverse momentum of the cluster-pointing COT track, $E / p<2$ unless track $p_{t}>50 \mathrm{GeV}$

6. number of COT axial or stereo track segments that consist of at least 5 hits each, $N_{a x} \geq 3$ and $N_{s t} \geq 2$;

7. track fiducial to the instrumented CEM region, i.e. $\left|x_{\mathrm{CES}}\right|<21 \mathrm{~cm}$ and $9<$ $\left|z_{\mathrm{CES}}\right|<230 \mathrm{~cm}$ in the CES local coordinate frame;

8. the seed tower must not be in the chimney region;

9. $z$ position of the extrapolated COT track at the closest approach to the beamline, $\left|z_{0}\right|<60 \mathrm{~cm}$

10. lateral shower profile, $L_{s h r}<0.2$. The variable compares energy in the adjacentto-seed towers to energy in the seed tower, referring to test beam data results;

11. comparison of the CES shower profile in the longitudinal $r z$ plane to test beam data results, $\chi_{\text {strip }}^{2}<10$; 
12. cluster-track matching in the transverse $r \phi$ plane with the COT track extrapolated to the CES radius, $-3<q \Delta x<1.5 \mathrm{~cm}$. The cut is charge-asymmetric because of track curving due to the magnetic field;

13. cluster-track matching along the $z$ axis with the COT track extrapolated to the CES radius, $\left|\Delta z_{\mathrm{CES}, \mathrm{COT}}\right|<3 \mathrm{~cm}$;

14. the electron candidate must not be tagged as part of a photon conversion ${ }^{3}$;

15. calorimetric isolation fraction $<0.1$, where the variable is defined as

$$
\text { cal isoFrac } \equiv \frac{E_{t \text { em }+ \text { had }} \text { with } \Delta R<0.4}{E_{t} \text { of lepton }}
$$

15. track isolation fraction $<0.1$, where the variable is defined as

$$
\operatorname{trk} \text { isoFrac } \equiv \frac{\sum \text { track } p_{t} \text { with } \Delta R<0.4-\text { track } p_{t} \text { of lepton }}{\operatorname{track} p_{t} \text { of lepton }}
$$

where the radius, $\Delta R \equiv \sqrt{(\Delta \eta)^{2}+(\Delta \phi)^{2}}$, describes a cone from the interaction point to the cluster centroid for the electron and around the COT track for the muon.

Excluding the requirement on track isolation fraction, the studies of TCE ID efficiencies and TCE data/MC scale factors are documented in [37] and [38]. The TCE ID scale factors, $\mathrm{SF}_{\mathrm{TCE}}=1.03 \pm 0.02$ for $10<E_{t}<20 \mathrm{GeV}$ and $\mathrm{SF}_{\mathrm{TCE}}=0.996 \pm 0.005$ for $E_{t}>20 \mathrm{GeV}$, were applied to the TCE electrons in MC.

\footnotetext{
${ }^{3}$ Such conversion can be $e^{ \pm} \rightarrow e^{ \pm} \gamma \rightarrow e^{ \pm} e^{+} e^{-}$which forms a trident track pattern or $\gamma \rightarrow e^{+} e^{-}$ which forms a $\Upsilon$-like track pattern. Electrons from photon conversion are usually soft, especially when compared to the original electron in the trident case.
} 


\subsubsection{Phoenix Electrons}

Forward electron identification, using the low- $\eta$ part of the PEM, relies on the so-called Phoenix tracking algorithm (PHX) [39] that attaches silicon hits to a COT track seeded by a PEM cluster to improve cluster-matching track quality since the COT track coverage is only partial while the silicon track coverage is complete in the forward region. An electron identified using the PHX tracking algorithm is called a Phoenix electron.

A Phoenix electron was selected with the following cuts:

1. there must be a cluster in either east or west PEM within a rapidity range of $1.1<\left|\eta_{d e t}\right|<3.6$

2. rapidity as measured by PES, $1.2<\left|\eta_{\text {det } \text { PES } 2 \mathrm{D}}\right|<2.0$;

3. corrected transverse energy ${ }^{4}, E_{t}>20 \mathrm{GeV}$;

4. ratio of hadronic to electromagnetic calorimetric energy deposit in the cluster, $E_{\text {had }} / E_{\text {em }}<0.05$

5. number of towers used in the PEM $3 \times 3$ cluster fit with the formula from test beam data, $N_{\text {PEM } 3 \times 3 \text { fit towers }} \neq 0$;

6. $\chi^{2}$ of the PEM $3 \times 3$ cluster fit, $\chi_{\text {PEM } 3 \times 3 \text { fit }}^{2}<10$;

7. ratio of energy in the central 5 to the total 9 scintillator strips in the PES U-layer, $U_{\mathrm{PES} 5 / 9}>0.65$

\footnotetext{
${ }^{4} \mathrm{PHX}$ energy correction was done with $m_{Z \rightarrow \mathrm{TCE}}+\mathrm{PHX}=91.2 \mathrm{GeV}$ calibration; overall speaking, $+4 \%$ in data and $+2 \%$ in MC.
} 
8. ratio of energy in the central 5 to the total 9 scintillator strips in the PES V-layer, $V_{\mathrm{PES} 5 / 9}>0.65$

9. track matching to the PEM cluster found according to the PHX tracking algorithm;

10. number of silicon hits composing the PHX track, $N_{S i \text { hits }} \geq 3$;

11. track $\left|z_{0}\right|<60 \mathrm{~cm}$;

12. cluster-track matching in the transverse $r \phi$ plane, $\Delta r_{\mathrm{PES}, \mathrm{PHX}}<3 \mathrm{~cm}$;

13. calorimetric isolation fraction $<0.1$.

The studies of PHX ID efficiencies, PHX data/MC ID scale factor and PHX charge fake rate $(\mathrm{QFR})$ are documented in [37]. The PHX ID scale factor, $\mathrm{SF}_{\mathrm{PHX}}=$ $0.948 \pm 0.016$, was applied to the PHX electrons in MC. Due to the limitation on forward track quality, charge misidentification in the PHX electron was not negligible. The PHX charge identification (QID) data/MC scale factor, defined as

$$
\mathrm{SF}_{\mathrm{PHX} \text { QID }} \equiv \frac{1-\mathrm{QFR} \text { in data }}{1-\mathrm{QFR} \text { in } \mathrm{MC}}
$$

\begin{tabular}{|c||c|c|c|}
\hline \hline detector rapidity & QFR in data & QFR in MC & SF $_{\text {PHX QID }}$ \\
\hline \hline $1.2 \leq\left|\eta_{\text {PES }}\right| \leq 1.4$ & $0.061 \pm 0.005$ & $0.061 \pm 0.002$ & $1.000 \pm 0.006$ \\
\hline $1.4<\left|\eta_{\text {PES }}\right| \leq 1.6$ & $0.099 \pm 0.007$ & $0.097 \pm 0.002$ & $0.998 \pm 0.008$ \\
\hline $1.6<\left|\eta_{\text {PES }}\right| \leq 1.8$ & $0.150 \pm 0.009$ & $0.143 \pm 0.003$ & $0.992 \pm 0.011$ \\
\hline $1.8<\left|\eta_{\text {PES }}\right| \leq 2.0$ & $0.168 \pm 0.011$ & $0.138 \pm 0.003$ & $0.965 \pm 0.013$ \\
\hline \hline
\end{tabular}

Table 5.1: PHX charge fake rate and PHX charge identification scale factor as a function of detector rapidity [37]. 
was applied to the PHX electrons in MC in addition to the PHX ID scale factor. The PHX charge misidentification rate in data and in MC and the PHX QID data/MC scale factor as a function of detector rapidity are listed in Table 5.1.

\subsubsection{Muons}

A muon seldom interacts with material and has a relatively long lifetime. As a minimum ionizing particle (MIP) traversing the CDF II detector, a muon leaves an isolated track in the silicon tracker and the COT, deposits small amounts of energy in the calorimeters and the shielding steel and leaves a stub in the outer muon detector(s) unless it goes through a gap between chambers.

Muon reconstruction and identification is implemented throughout the space of $|\eta|<1$; it starts from MIP identification and finishes with the matching of the MIP track to a muon stub or muon stubs. Every muon is type-defined by the muon detector(s) it matches to. The trigger efficiency and the data/MC scale factor, as well as the commission run period, differ from one muon detector to another.

The cosmic ray event tagger is described in [40]. In this physics data analysis, cosmic rays were regarded as a source of background. Muons were required to come from events not tagged as cosmic.

\subsubsection{Minimum Ionizing Particles}

As the basis of any further muon selection, a minimum ionizing particle (MIP) was identified with the following cuts:

1. event not tagged as cosmic; 
2. transverse momentum ${ }^{5}, p_{t}>10 \mathrm{GeV}$;

3. number of COT axial or stereo track segments that consist of at least 5 hits each, $N_{a x} \geq 3$ and $N_{s t} \geq 2$;

4. $\operatorname{track}\left|z_{0}\right|<60 \mathrm{~cm}$;

5. impact parameter, i.e. distance at the closest approach from the extrapolated COT track to the primary vertex:

(a) $\left|d_{0}\right|<0.02 \mathrm{~cm}$ for a silicon+COT track with $p_{t}>20 \mathrm{GeV}$;

(b) $\left|d_{0}\right|<0.2 \mathrm{~cm}$ for the rest;

6. energy deposit in the electromagnetic calorimeter:

(a) for track $p_{t}<20 \mathrm{GeV}, E_{e m}<2 \mathrm{GeV}$;

(b) for track $p_{t}>20 \mathrm{GeV}, E_{\text {em }}<2+\max [0,0.0115 \times(p-100)] \mathrm{GeV}$;

7. energy deposit in the hadronic calorimeter:

(a) for track $p_{t}<20 \mathrm{GeV}, E_{\text {had }}<3.5+0.125 \times p_{t} \mathrm{GeV}$;

(b) for track $p_{t}>20 \mathrm{GeV}, E_{\text {had }}<6+\max [0,0.028 \times(p-100)] \mathrm{GeV}$;

8. calorimetric isolation fraction $<0.1$;

9. track isolation fraction $<0.1$;

\footnotetext{
${ }^{5}$ To a fairly good approximation, MIP energy is the same as its momentum as measured using
} track curvature in COT due to the solenoid's magnetic field. 
10. radius of the COT track extrapolated to the COT end $z$ position, COT exit radius $\rho_{\mathrm{COT}}>140 \mathrm{~cm}$. Since the CMU and CMP detectors are within $\left|\eta_{\text {det }}\right| \lesssim$ 0.65, such a cut (effectively $\left|\eta_{\text {det }}\right|<0.9551432$ ) has an effect only on CMX and CMIO muons.

\subsubsection{CMUP Muons}

A muon as a MIP attached with a stub in the CMU detector and another stub in the CMP detector (CMUP) was selected with the following additional cuts:

11. track pointing to the instrumented CMU and CMP detector regions in the transverse $(x \equiv r \phi)$ plane and the longitudinal $(z \equiv r z)$ plane with outward distances from the nearest detector edge (fiducial distances):
(a) $x$-fid CMU $_{\mathrm{CM}}<0$ and $z$-fid $\mathrm{CMU}_{\mathrm{CM}}<0$;

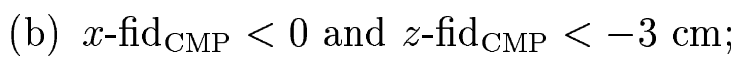

12. track-stub matching in the transverse plane with the COT track extrapolated to the CMU and CMP muon detector radii:
(a) $\Delta x_{\mathrm{CMU}}<3 \mathrm{~cm}$
(b) $\Delta x_{\mathrm{CMP}}<5 \mathrm{~cm}$;

13. run number $\geq 154449$ because stacks 6 -12 in the CMP top sector (a.k.a. bluebeam section) have functioned stably only since run 154449.

Excluding the requirement on track isolation fraction, the studies of CMUP reconstruction and identification efficiencies and CMUP data/MC scale factors are 
documented in [34] and [41]. The CMUP REC and ID scale factors, $\mathrm{SF}_{\mathrm{CMUP}}=$ $0.85 \pm 0.05$ for $10<p_{t}<20 \mathrm{GeV}$ and $\mathrm{SF}_{\mathrm{CMUP}}=0.892 \pm 0.009$ for $p_{t}>20 \mathrm{GeV}$, were applied to the CMUP muons in MC.

\subsubsection{CMX Muons}

A muon as a MIP attached with a stub in the CMX detector (CMX) was selected with the following additional cuts:

11. track pointing to the instrumented CMX detector region with fiducial distances,

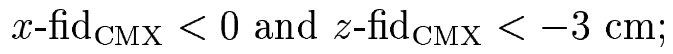

12. track-stub matching in the transverse plane with the COT track extrapolated to the CMX muon detector radius, $\Delta x_{\mathrm{CMX}}<6 \mathrm{~cm}$;

13. run number $\geq 150145$ because CMX arches (east and west wedges 21-23, 0-4 and $7-14)^{6}$ have served in data taking only since run 150145 .

Excluding the requirement on track isolation fraction, the studies of CMX reconstruction and identification efficiencies and CMX data/MC scale factors are documented in [34] and [41]. The CMX REC and ID scale factors, $\mathrm{SF}_{\mathrm{CMX}}=0.90 \pm 0.05$ for $10<p_{t}<20 \mathrm{GeV}$ and $\mathrm{SF}_{\mathrm{CMX}}=0.999 \pm 0.006$ for $p_{t}>20 \mathrm{GeV}$, were applied to the CMX muons in MC.

${ }^{6} \mathrm{CMX}$ miniskirt (east and west wedges 15-20) and keystone (west wedges 5-6) had not served stably until the restart of data taking in January 2005. 


\subsubsection{CMU-only Muons}

A muon as a MIP attached with a stub in the CMU detector but not a stub in the CMP detector (CMU) was selected with the following additional cuts:

11. track pointing to the instrumented CMU detector region with fiducial distances, $x$-fid ${ }_{\mathrm{CMU}}<0$ and $z$-fid CMU $_{\mathrm{C}}<0$;

12. track-stub matching in the transverse plane with the COT track extrapolated to the $\mathrm{CMU}$ muon detector radius, $\Delta x_{\mathrm{CMU}}<3 \mathrm{~cm}$;

13. $\operatorname{track} p_{t}>20 \mathrm{GeV}$;

14. number of COT stereo track segments that consist of at least 5 hits each, $N_{s t} \geq 3$.

The additional cuts on MIP transverse momentum and COT stereo track segments were made to suppress the CMU muon fake rate.

Excluding the requirement on track isolation fraction, the studies of CMU reconstruction and identification efficiencies and data/MC scale factors are documented in [34]. The CMU REC and ID scale factors, $\mathrm{SF}_{\mathrm{CMU}}=0.889 \pm 0.010$ for $p_{t}>20 \mathrm{GeV}$, were applied to the CMU muons in MC.

\subsubsection{CMP-only Muons}

A muon as a MIP attached with a stub in the CMP detector but not a stub in the CMU detector (CMP) was selected with the following additional cuts:

11. track pointing to the instrumented CMP detector region with fiducial distances, $x$-fid ${ }_{\mathrm{CMP}}<0$ and $z$-fid ${ }_{\mathrm{CMP}}<-3 \mathrm{~cm}$; 
12. track-stub matching in the transverse plane with the COT track extrapolated to the CMP muon detector radius, $\Delta x_{\mathrm{CMP}}<5 \mathrm{~cm}$;

13. $\operatorname{track} p_{t}>20 \mathrm{GeV}$;

14. number of COT stereo track segments that consist of at least 5 hits each, $N_{s t} \geq 3$;

15. run number $\geq 154449$ due to the CMP bluebeam section.

The additional cuts on MIP transverse momentum and COT stereo track segments were made to suppress the CMP muon fake rate.

Excluding the requirement on track isolation fraction, the studies of CMP reconstruction and identification efficiencies and data/MC scale factors are documented in [34]. The CMP REC and ID scale factors, $\mathrm{SF}_{\mathrm{CMP}}=0.907 \pm 0.009$ for $p_{t}>20 \mathrm{GeV}$, were applied to the CMP muons in MC.

\subsubsection{Stubless Muons}

A muon as a MIP attached with no stub as it traverses a muon detector gap, i.e. a stubless central muon inside-out track (CMIO), was selected with the following additional cuts:

11. number of COT stereo track segments that consists of at least 5 hits, $N_{s t} \geq 3$;

12. energy deposit in the calorimeters, $E_{\text {em+had }}>0.1 \mathrm{GeV}$.

A minimum calorimetric energy deposit of MIP was required to suppress the CMIO muon fake rate.

Excluding the requirement on track isolation fraction, the studies of CMIO reconstruction and identification efficiencies and data/MC scale factors are documented 
in [34]. The CMIO REC and ID scale factor, $\mathrm{SF}_{\mathrm{CMIO}}=0.995 \pm 0.005$ for $p_{t}>20 \mathrm{GeV}$, was applied to the CMIO muons in MC.

For all lepton types except PHX track isolation fraction and the cut efficiency of trk isoFrac $<0.1$ were studied and, as shown in Figure 5.1, the agreement between data and MC was observed to be very good. Therefore, for the trk isoFrac $<0.1$ cut no data/MC scale factors were applied; however, extra systematic uncertainty is quoted.

\subsubsection{Trigger Leptons}

After all the leptons were identified, an event was required to have at least one TCE, PHX, CMUP or CMX lepton with $E_{t}>20 \mathrm{GeV}$ to meet the trigger requirement. An event was further required to have corrected missing transverse energy $\not_{t}>25 \mathrm{GeV}$ if a PHX electron in it had to be the trigger lepton.

\subsection{Jets}

Jets are gluon or quark fragmentation, which may leave non-isolated tracks, depending on hadron flavor and energy. The production cross-section of jets is large, in millibarns at the Tevatron, especially from gluons and light quarks or at low energy.

A jet deposits most of its energy in the calorimeters - typically $10 \%-30 \%$ in the electromagnetic one and the rest in the hadronic one. Extremely hard jets not stopped by all the energy absorbing lead and iron in the calorimeters and the steel in front of the muon chambers are called "hadronic punch-through"; this kind of jets may be misidentified as muons, the treatment to which is described in Chapter 6 . 

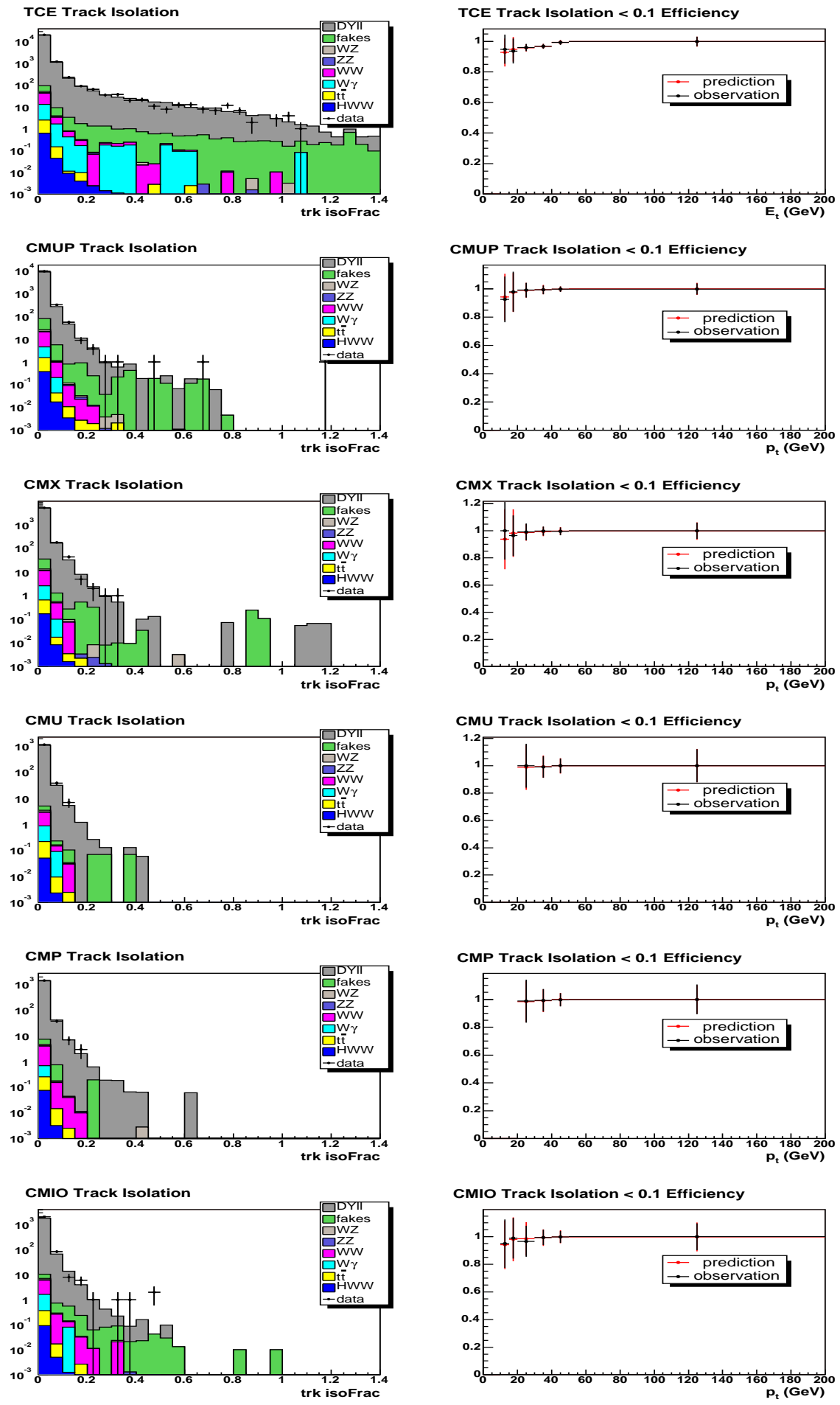

Figure 5.1: Lepton track isolation fraction distributions and cut efficiencies of trk isoFrac $<0.1$ for each type of leptons that required track isolation. 
Calorimetric towers deposited with jet energies often form a continuum over multiple jets, which makes the jet clustering result algorithm-dependent.

\subsubsection{Clustering Algorithm}

The JetClu cone clustering algorithm has been in extensive use at CDF since Run I. Other jet clustering algorithms, such as midpoint, seedless or even $k_{T}$, have been studied in Run II to overcome flaws of JetClu, such as ratcheting ${ }^{7}$ and lack of infrared safety $^{8}$, although many of them have been proved no silver bullet for correcting these flaws.

JetClu with cone size $\Delta R=0.4$ was used in this analysis, with the clustering algorithm as described below:

1. calorimetric towers with $E_{t} \equiv E_{e m} \sin \theta_{e m}+E_{\text {had }} \sin \theta_{\text {had }}>1 \mathrm{GeV}$ are enlisted as seed towers;

2. starting from the highest- $E_{t}$ one, adjacent seed towers within $\Delta R$ are clumped together to form preclusters. Each seed tower is exclusively assigned to one precluster;

3. calorimetric towers with $E_{t}>0.1 \mathrm{GeV}$ within $\Delta R$ from the $E_{t}$-weighed precluster centroids are clumped to form clusters. Iteratively, cluster centroids are recalculated and unclumped towers within $\Delta R$ of new centroids are clumped

\footnotetext{
${ }^{7} \mathrm{~A}$ tower is never removed from the cluster it clumps to even if the centroid has drifted away and the tower has become out of cone.

${ }^{8} \mathrm{~A}$ soft gluon in the cone overlapping region of otherwise two jets can combine them into one jet, for example.
} 
until the tower list remains unchanged or the number of iterations reaches the maximum;

4. overlap fraction is calculated for any two overlapping clusters as sum $E_{t}$ of common towers divided by $E_{t}$ of the lower- $E_{t}$ cluster. If the overlap fraction is above a cutoff, 0.75 for example, the clusters are combined; otherwise common towers are reclumped to the cluster of nearest centroid;

5. clusters in the final list are regarded as raw jets.

\subsubsection{Energy Correction}

Jet energy correction at CDF II has been probed from three jet fragmentation levels, as shown in Figure 5.2, and developed into seven energy correction levels, as described below, to accommodate different effects that distort calorimetric measurements of jet energy:

Level 0 - online and offline calibrations with scale factors, linear energy responses, ${ }^{137} \mathrm{Cs}$ sources, MIP energy deposits and the dilepton invariant mass peak of $Z \rightarrow e e$ decays;

Level 1 - based on transverse energy conservation of two-to-two processes, a rapidity-dependent "relative" correction is applied to scale the raw jet energy outside the off-crack central calorimeter region of $0.2<|\eta|<0.6$ to inside the region;

Level 2 - not in use; 
Level 3 - a "raw-scale" correction to account for the difference between Run I and Run II absolute corrections;

Level 4 - a correction for the energy increase due to multiple interactions, derived from minimum bias data and parameterized as a function of number of vertices;

Level 5 - an "absolute" correction for any non-linearity or energy loss in the uninstrumented calorimetric regions, including underlying event energy subtraction, to the sum $p_{t}$ of particles within the clustering cone around the parton and the matching jet;

Level 6 - a correction meant to account for the difference between Run I and Run II underlying event energy subtractions from particle-level jet energies;

Level 7 - Run I out-of-cone correction in $0.4<\Delta R<1.3$ to account for particle-

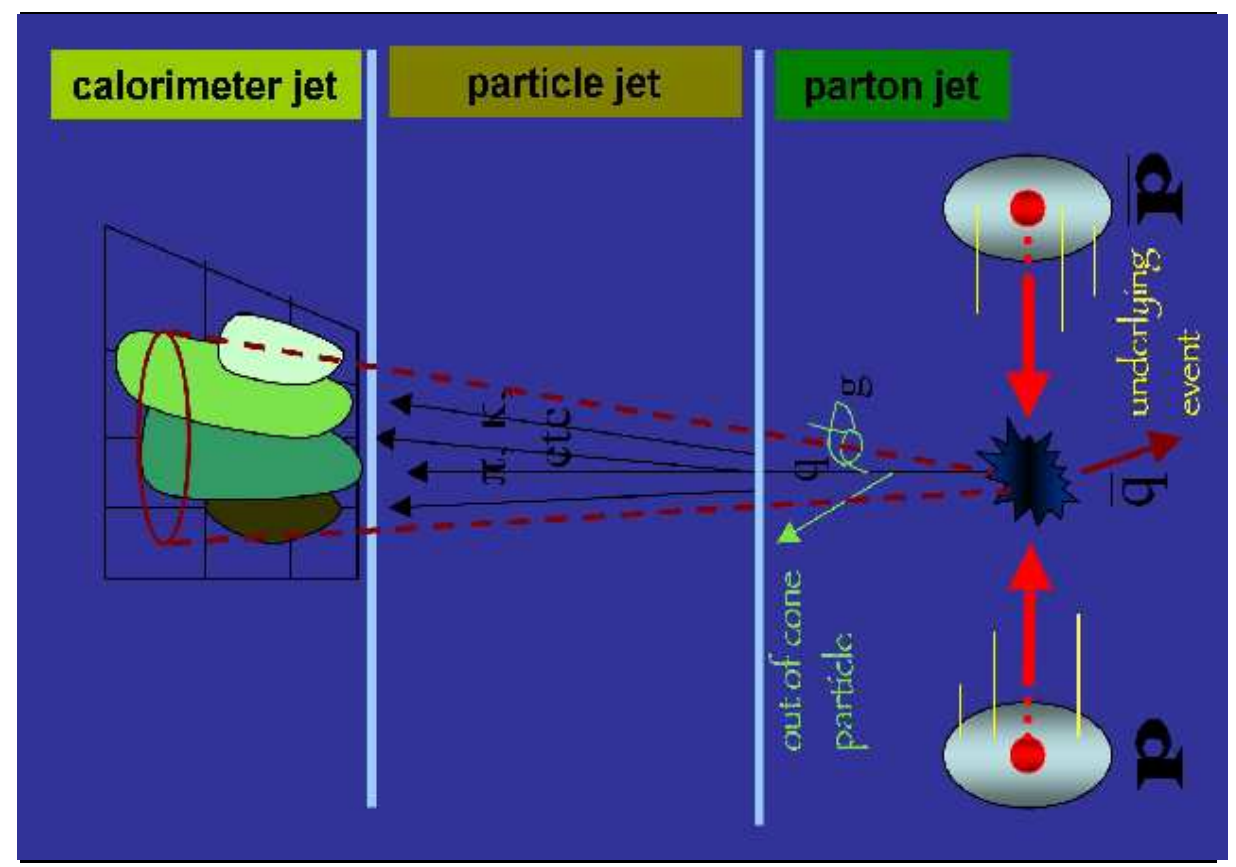

Figure 5.2: Jet fragmentation levels for the CDF II jet energy correction. 
level jet energy leakage.

Depending on the physics concern, jet energy can be corrected up to any of these seven levels.

Jet energy for this analysis was corrected up to Level 5 and the uncertainty due to such correction was estimated using the JetUser package of jetCorr04b tag [42].

\subsubsection{Jets for Veto Consideration}

Jets were selected with the cuts of corrected transverse energy $E_{t}>15 \mathrm{GeV}$ and detector rapidity $\left|\eta_{\text {det }}\right|<2.5$ for later veto consideration.

\subsection{Missing Transverse Energy}

In the simplest definition, missing transverse energy $\left(\not_{t}\right)$ is a calorimetric energy imbalance of the event in a plane transverse to the beamline under the law of energy conservation.

Missing transverse energy may be due to neutrinos or perhaps some exotic particles that do not interact with any detector material at all; it may be due to instrumental reasons, such as leptons or jets flying into detector gaps, imperfect calorimetric energy measurement or offline reconstruction.

Missing transverse energy was corrected by the following levels prior to its service in event selection:

1. raw $\mathbb{E}_{t}$ taken directly from the imbalance of event total transverse energy, as measured by the calorimeters;

2. corrected for the location of primary vertex; 
3. corrected for the identified muon(s);

4. corrected for the offline jet energy correction at a level $\geq 5$;

5. corrected for the offline PHX energy correction;

6. corrected for the very slight MC-only TCE energy correction. 


\section{Chapter 6}

\section{Estimate of Lepton Fake Rates}

Leptons can be faked by jets ${ }^{1}$ and this introduces what is formally called $\mathrm{QCD} / W+$ jet and informally called fake background into the search for events with dilepton plus large $\not_{t}$ signature when events containing fake leptons pass the selection criteria and get misidentified as signal events.

The rates at which leptons would get faked by jets, i.e. the lepton fake rates, were small. However, the jet production cross-section was so large in comparison to the signal that the fake background due to jets was not negligible. Because the lepton fake rates were small, the total fake background could be acceptably approximated by the single fake lepton part of $\mathrm{it}^{2}$.

\footnotetext{
${ }^{1}$ Electrons additionally can be faked by photons. The treatments to photon and jet fake backgrounds are different because of photon and jet MC modeling qualities.

${ }^{2} \mathrm{~A}$ fake dilepton event can contain a single fake lepton and a real lepton that is primarily from the largest cross-sectioned single lepton production process, $W \rightarrow \ell \nu$, or two fake leptons.
} 


\subsection{QCD $/ W+$ jet Background Estimate Procedure}

Estimations of other fake background, including photon fake background, can be achieved through MC. However, the jet fake background was estimated entirely with real data instead of $\mathrm{MC}$ because jet fakes were not modeled well enough in $\mathrm{MC}$, as always, especially on jet track-stub matching and isolation in PYTHIA.

The $\mathrm{QCD} / W+$ jet background was estimated in the following procedure:

1. the rate at which leptons were faked by jets was estimated per lepton type with jet data. The data from jet triggers are jet-rich and lepton-poor and hence good for estimates of the lepton fake rates;

2. events in high- $p_{t}$ lepton data samples, where a signal was sought, were tested to see if they would pass the selection criteria assuming one or two jets faking leptons;

3. fakeable objects, defined as jets that would get events selected when faking leptons, were weighed with the lepton fake rates. Those combined weights were summed up to be the QCD/W+jet background.

\subsubsection{Lepton Fake Rate Estimate}

The estimation of the lepton fake rates is documented in [43].

As the uncertainty in the $\mathrm{QCD} / W+$ jet background was the highest among all the backgrounds in this analysis, it was desired to estimate the lepton fake rates and the $\mathrm{QCD} / W+$ jet background with the smallest achievable systematic uncertainty. 


\begin{tabular}{|c|l|l|}
\hline \hline DFC ID & trigger & trigger requirement \\
\hline \hline bhel0d & CEM18 & at least a central electron with $E_{t}>18 \mathrm{GeV}$ \\
\hline bpel0d & MET_PEM & $\begin{array}{l}\text { at least a plug electron with } E_{t}>20 \mathrm{GeV} \\
\text { and missing transverse energy } \not_{t}>15 \mathrm{GeV}\end{array}$ \\
\hline bhmu0d & $\begin{array}{l}\text { CMUP18 } \\
\text { CMX18 }\end{array}$ & $\begin{array}{l}\text { at least a CMUP muon with } p_{t}>18 \mathrm{GeV} \\
\text { at least a CMX muon with } p_{t}>18 \mathrm{GeV}\end{array}$ \\
\hline \hline gjt10d & Jet20 & at least a raw jet with $E_{t}>20 \mathrm{GeV}$ and $|\eta|<3.6$ \\
\hline gjt20d & Jet50 & at least a raw jet with $E_{t}>50 \mathrm{GeV}$ and $|\eta|<3.6$ \\
\hline gjt30d & Jet70 & at least a raw jet with $E_{t}>70 \mathrm{GeV}$ and $|\eta|<3.6$ \\
\hline gjt40d & Jet100 & at least a raw jet with $E_{t}>100 \mathrm{GeV}$ and $|\eta|<3.6$ \\
\hline \hline
\end{tabular}

Table 6.1: High $p_{t}$ lepton and jet datasets and trigger requirements used for the lepton fake rate estimate. For the jet triggers, requirements are made with the JetClu cone size $\Delta R=0.7$ clustering algorithm.

\subsubsection{Datasets}

Data samples from four prescaled jet inclusive triggers, as listed in Table 6.1, were made into topNt 5.3.3_nt [50] ntuples for the lepton fake rate estimate.

The DQM good run list 1141 version 7.0 was applied, requiring the silicon, CMU, CMP and CMX detectors in good conditions with the CMX bit ignored for run $<$ 150145. The total offline integrated luminosity after the application of the good run list was $\mathcal{L}_{\text {int }}=320 \pm 19 \mathrm{pb}^{-1}$.

\subsubsection{Fake Rate Definitions}

A lepton fake rate is defined as the probability of a fakeable object passing the lepton identification criteria. Such a lepton fake rate is highly dependent on the definition for a fakeable object and, since object composition varies from sample to sample, balance should be sought between a generalized fakeable object definition and consistent lepton fake rates among samples. 
For electrons, the fakeable objects were electromagnetic clusters loosely selected from CdfEmObjects, which have a higher electromagnetic fraction than generic jets and hence a higher chance to fake electrons. The energy measurement of the electromagnetic calorimeters has a substantially smaller uncertainty than that of the hadronic calorimeters. Another merit is that the electromagnetic clusters do not have any quark/gluon-like substructure to complicate the study.

For muons, the fakeable objects were minimum ionizing particles loosely selected from CdfMuons. This kind of fakeable objects encompasses all the real or fake lepton sources and allows easy $p_{t}$ parameterization.

Mathematically, a lepton fake rate can be written as the number of fakeable objects passing the lepton selection cuts divided by the number of fakeable objects

$$
\begin{aligned}
\mathrm{FR}_{\mathrm{TCE} / \mathrm{PHX}} & \equiv \frac{\mathrm{TCE} / \mathrm{PHX} \text { fake electrons }}{\text { central/plug emObjects }} \\
\mathrm{FR}_{\mathrm{CMUP} / \mathrm{X} / \mathrm{U} / \mathrm{IO}} & \equiv \frac{\mathrm{CMUP} / \mathrm{X} / \mathrm{U} / \mathrm{P} / \mathrm{IO} \text { fake muons }}{\mathrm{CMUP} / \mathrm{X} / \mathrm{U} / \mathrm{P} / \mathrm{IO} \text { MIP tracks }}
\end{aligned}
$$

in the jet samples.

\subsubsection{Lepton Selections}

The lepton selection cuts for numerators, which are the same as for the main analysis [44], are listed in Table 6.2, Table 6.4 and Table 6.5.

\subsubsection{Fakeable Object Selections}

The selection cuts for denominator fakeable objects are listed in Table 6.3 and Table 6.6. 
CdfEmObjects from higher- $E_{t}$ jet triggers tend to have larger hadronic fractions due to the difference in trigger and offline clustering algorithms. Therefore, the requirement of $E_{\text {had }} / E_{\text {em }}<0.125$ improves the consistency in electron fake rate denominator composition among different jet samples. The effect of requiring the electron fake rate denominators $E_{\text {had }} / E_{\text {em }}<0.125$ is shown with the CdfEmObject $E_{\text {had }} / E_{\text {em }}$ profiles vs. cal isoFrac in Figure 6.2.

CdfMuons include electrons, conversions, hadronic punches-throughs and decaysin-flight as well as muons. The CdfMuons generally deposit more energy in the calorimeters than the MIPs since there is no minimum ionizing requirement. Therefore, the requirement of $E_{\text {em+had }} / p<1$ improves the consistency in muon fake rate denominator composition among different jet samples. The effect of requiring muon fake rate denominators $E_{h a d+e m} / p<1$ is shown with the MIP $E_{\text {em+had }} / p$ profiles vs. cal isoFrac in Figure 6.3.

The high- $p_{t}$ lepton data samples are primarily composed of $W+$ jet and tend to have good calorimetric isolation. Therefore, requiring all the lepton fake rate denominators to have cal isoFrac $<0.2$, we further improved the consistency in lepton fake rate denominator composition among different jet samples, particularly in the electron cases, while keeping the applicability of the estimated lepton fake rates to the fakeable objects found in the signal data samples. The fake rate denominator cal isoFrac profiles vs. $E_{t}$ before and after such a requirement for each type of lepton are shown in Figure 6.4 and Figure 6.5.

Improving the fake rate denominator composition consistency among the jet samples improves the fake rate consistency among the jet samples. 

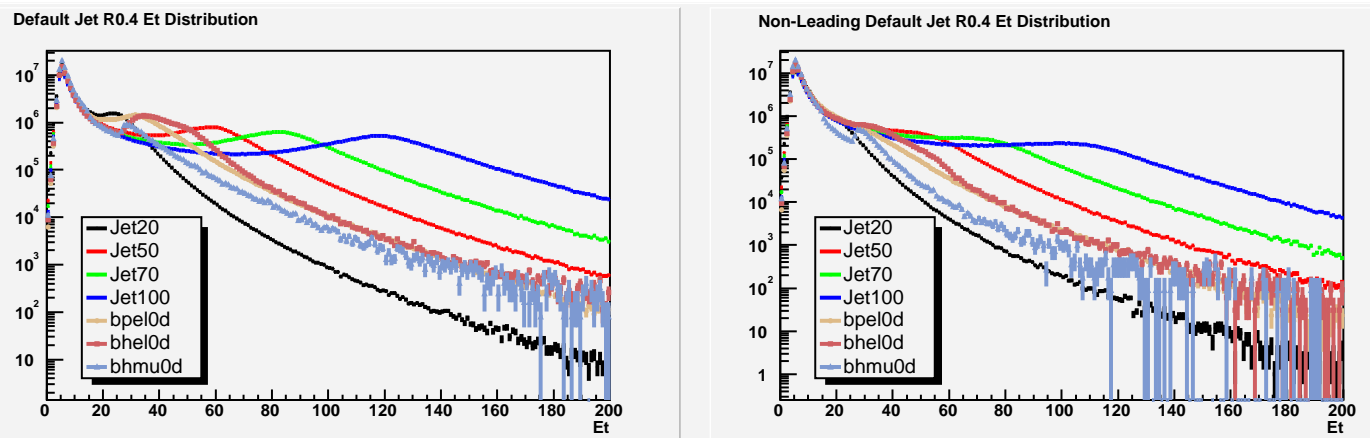

Figure 6.1: $E_{t}$ distributions of jets in the high $p_{t}$ lepton and jet samples before (left) and after (right) removing the highest- $E_{t}$ jets.

\subsubsection{Trigger Bias Reduction}

To reduce trigger bias we removed the highest- $E_{t}$ jets (default CdfJets, cone size $\Delta R=0.4)$ and all the numerator and denominator objects within their cones. We did not completely remove the trigger bias. However, the remnant trigger bias propagated little into the denominator fakeable objects, as shown in Figure 6.7, so the lepton fake rates were not affected much by the trigger bias.

\subsubsection{Results}

The $p_{t}$ distributions of numerator leptons and the $E_{t}$ distributions of denominator objects from the jet samples are shown in Figure 6.6 and Figure 6.7. The lepton fake rate results are shown in Figure 6.8. Bayesian errors were included for the bins that lacked of numerator statistics [45].

We compared the number of leptons observed in each jet sample with the number of leptons predicted using Jet20/50/70/100 fake rates and found overall agreement, as shown in Table 6.7 and Table 6.8 . 


\begin{tabular}{|l|}
\hline \hline CollType $=$ DefEm \\
\hline region $=0$ (central) \\
\hline$E_{t}>10 \mathrm{GeV}$ \\
\hline$E_{\text {had }} / E_{e m}<0.055+0.00045 E$ \\
\hline$E / p<2$ or track $p_{t}>50 \mathrm{GeV}$ \\
\hline$L_{s h r}<0.2$ \\
\hline$-3<Q \cdot \Delta x<1.5 \mathrm{~cm}$ \\
\hline$\left|\Delta z_{\mathrm{CES}}\right|<3 \mathrm{~cm}$ \\
\hline$\left|z_{0}\right|<60 \mathrm{~cm}$ \\
\hline$\chi_{\text {strip }}^{2}<10$ \\
\hline number of good axial SL segments $\geq 3$ \\
\hline number of good stereo SL segments $\geq 2$ \\
\hline conversion $\neq 1$ \\
\hline fiducial $=1$ \\
\hline calorimetric isolation fraction $<0.1$ \\
\hline track isolation fraction $<0.1$ \\
\hline \hline
\end{tabular}

Table 6.2: Selection cuts for tight central electrons (TCE).

\begin{tabular}{|l|}
\hline \hline CollType $=$ DefEm \\
\hline region $=0$ (central $)$ \\
\hline$E_{t}>10 \mathrm{GeV}$ \\
\hline$E_{\text {had }} / E_{\text {em }}<0.125$ \\
\hline track $p_{t}>0$ \\
\hline conversion $\neq 1$ \\
\hline cal isoFrac $<0.2$ \\
\hline \hline
\end{tabular}

\begin{tabular}{|l|}
\hline \hline CollType $=$ DefEm \\
\hline region $=1$ (plugs) \\
\hline$E_{t}>10 \mathrm{GeV}$ \\
\hline$E_{\text {had }} / E_{\text {em }}<0.125$ \\
\hline$|\mathrm{PES} 2 \mathrm{D} \eta|>1.2$ \\
\hline$|\mathrm{PES} 2 \mathrm{D} \eta|<2.0$ \\
\hline cal isoFrac $<0.2$ \\
\hline \hline
\end{tabular}

Table 6.3: Selection cuts for central (left) and plug (right) fakeable electromagnetic objects, i.e. $\mathrm{FR}_{\mathrm{TCE}}$ (left) and $\mathrm{FR}_{\mathrm{PHX}}$ (right) denominators. 


\begin{tabular}{|l|}
\hline \hline CollType $=$ Phoenix \\
\hline region $=1$ (plugs) \\
\hline$E_{t}>10 \mathrm{GeV}$ \\
\hline$E_{\text {had }} / E_{\text {em }}<0.05$ \\
\hline PEM $3 \times 3$ fit tower $\neq 0$ \\
\hline PEM $3 \times 3 \chi^{2}<10$ \\
\hline PES $2 \mathrm{D} 5 \times 9 \mathrm{U}>0.65$ \\
\hline PES $2 \mathrm{D} 5 \times 9 \mathrm{~V}>0.65$ \\
\hline $1.2<\mid$ PES $2 \mathrm{D} \eta \mid<2.0$ \\
\hline calorimetric isolation fraction $<0.1$ \\
\hline phxMatch TRUE \\
\hline number of silicon hits for PHX track $\geq 3$ \\
\hline $\mid z_{0}($ PHX track $) \mid<60$ cm \\
\hline$\Delta R($ PHX track, PES cluster $)<3 \mathrm{~cm}$ \\
\hline \hline
\end{tabular}

Table 6.4: Selection cuts for phoenix electrons (PHX).
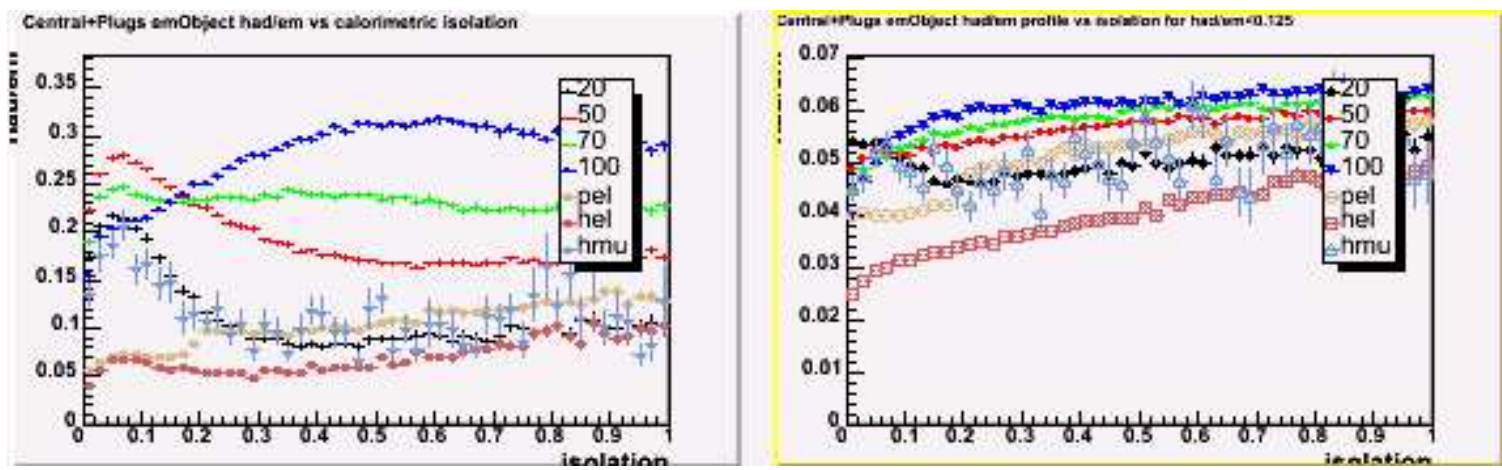

Figure 6.2: $E_{\text {had }} / E_{\text {em }}$ profiles vs. cal isoFrac of electromagnetic objects in the high $p_{t}$ lepton and jet samples before (left) and after (right) requiring $E_{\text {had }} / E_{\text {em }}<0.125$. 


\begin{tabular}{|c|c|c|}
\hline \multicolumn{3}{|c|}{ for CMUP/CMX/CMIO $p_{t}>10 \mathrm{GeV}$} \\
\hline \multicolumn{3}{|c|}{ for $\mathrm{CMU} / \mathrm{CMP} \quad p_{t}>20 \mathrm{GeV}$} \\
\hline \multirow[t]{10}{*}{ for all muons } & \multicolumn{2}{|c|}{ non-cosmic } \\
\hline & \multicolumn{2}{|c|}{$E_{e m}<2+\left(p_{t}>20 \mathrm{GeV}\right) \max (0,0.0115(p-100)) \mathrm{GeV}$} \\
\hline & \multicolumn{2}{|c|}{$E_{\text {had }}<6-\left(p_{t}<20 \mathrm{GeV}\right)\left(2.5-0.125 p_{t}\right)$} \\
\hline & \multicolumn{2}{|c|}{$+\left(p_{t}>20 \mathrm{GeV}\right) \max (0,0.028(p-100)) \mathrm{GeV}$} \\
\hline & \multirow{2}{*}{\multicolumn{2}{|c|}{$\begin{array}{l}\left|d_{0}\right|<0.02 \mathrm{~cm} \text { for tracks with silicon hits attached } \\
\left|d_{0}\right|<0.2 \mathrm{~cm} \text { for tracks without silicon hits attached }\end{array}$}} \\
\hline & & \\
\hline & \multicolumn{2}{|c|}{$\left|z_{0}\right|<60 \mathrm{~cm}$} \\
\hline & \multicolumn{2}{|c|}{ calorimetric isolation fraction $<0.1$} \\
\hline & \multicolumn{2}{|c|}{ track isolation fraction $<0.1$} \\
\hline & \multicolumn{2}{|c|}{ number of good axial SL segments $\geq 3$} \\
\hline \multicolumn{3}{|c|}{ for CMUP/CMX $\quad$ number of good stereo SL sę } \\
\hline \multicolumn{3}{|c|}{ for $\mathrm{CMU} / \mathrm{CMP} / \mathrm{CMIO}$} \\
\hline \multicolumn{3}{|c|}{ for $\mathrm{CMUP} / \mathrm{CMU} / \mathrm{CMP} \quad$ run number $\geq 154$} \\
\hline \multicolumn{3}{|c|}{ for $\mathrm{CMUP} / \mathrm{CMU} \quad \Delta x_{\mathrm{CMU}}<3 \mathrm{~cm}$} \\
\hline \multicolumn{3}{|c|}{ for CMUP/CMP $\quad \Delta x_{\mathrm{CMP}}<5 \mathrm{~cm}$} \\
\hline \multicolumn{3}{|c|}{ for CMUP $/$ CMP $\quad$ x-fid $(\mathrm{CMP})<0$ and $\mathrm{z}$-fid $(\mathrm{CMP})<-3 \mathrm{~cm}$} \\
\hline \multicolumn{3}{|c|}{\begin{tabular}{ll} 
for CMX & run number $>150145$ \\
\cline { 2 - 2 } &
\end{tabular}} \\
\hline \multicolumn{3}{|c|}{\begin{tabular}{ll}
\cline { 2 - 3 }$>186598$ or not from miniskirt and keystone \\
run $>$
\end{tabular}} \\
\hline \multicolumn{3}{|c|}{$\Delta x_{\mathrm{CMX}}<6 \mathrm{~cm}$} \\
\hline \multicolumn{3}{|c|}{ x-fid (CMX) $<0$ and $\mathrm{z}$-fid $(\mathrm{CMX})<-3 \mathrm{~cm}$} \\
\hline \multicolumn{3}{|c|}{ for CMXtriggerable $/ \mathrm{CMIO} \quad \rho_{\mathrm{COT}}>140 \mathrm{~cm}$} \\
\hline \multirow{3}{*}{\multicolumn{2}{|c|}{ for $\mathrm{CMIO}$}} & non-CMUP/CMX/CMU/CMP/BMU \\
\hline & & track fiducial $=4$ \\
\hline & & $E_{e m}+E_{h a d}>0.1 \mathrm{GeV}$ \\
\hline
\end{tabular}

Table 6.5: Selection cuts for CMUP/CMX/CMU/CMP/CMIO muons. 


\begin{tabular}{|c|c|c|}
\hline for $\mathrm{MIP}_{\mathrm{CMU}}$ & CMX/CMIO & $p_{t}>10 \mathrm{GeV}$ \\
\hline for $\mathrm{MIP}_{\mathrm{CMU}}$ & $\mathrm{MP}$ & $p_{t}>20 \mathrm{GeV}$ \\
\hline for all MIP & non-cosmi & \\
\hline & $\left|d_{0}\right|<0.0$ & cm for tracks with silicon hits attached \\
\hline & $\left|d_{0}\right|<0.2$ & m for tracks without silicon hits attached \\
\hline & $\left|z_{0}\right|<60$ & \\
\hline & $\left(E_{e m}+E_{l}\right.$ & $\left.d_{d}\right) / p<1$ \\
\hline & calorimetr & $\mathrm{c}$ isolation fraction $<0.2$ \\
\hline for $\mathrm{MIP}_{\mathrm{CMU}}$ & $/ \mathrm{CMU} / \mathrm{CMP}$ & run number $\geq 154449$ or not in bluebeam \\
\hline for $\mathrm{MIP}_{\mathrm{CMU}}$ & CMP & $\mathrm{x}$-fid(CMP) $<0$ and z-fid(CMP) $<-3 \mathrm{~cm}$ \\
\hline for $\mathrm{MIP}_{\mathrm{CMX}}$ & run $\mathrm{r}$ & umber $>150145$ \\
\hline & run : & 186598 or not from miniskirt and keystone \\
\hline & $\mathrm{x}$-fid & $\overline{\mathrm{CMX}})<0$ and $\mathrm{z}$-fid $(\mathrm{CMX})<-3 \mathrm{~cm}$ \\
\hline for $\mathrm{MIP}_{\mathrm{CMX}}$ & ggerable/CMIO & $\rho_{\mathrm{COT}}>140 \mathrm{~cm}$ \\
\hline for MIP $\mathrm{MMIC}_{\mathrm{C}}$ & & non-MIP $\mathrm{CMUP} / \mathrm{CMX} / \mathrm{CMU} / \mathrm{CMP} / \mathrm{BMU}$ \\
\hline
\end{tabular}

Table 6.6: Selection cuts for CMUP/CMX/CMU/CMP/CMIO fakeable MIP tracks

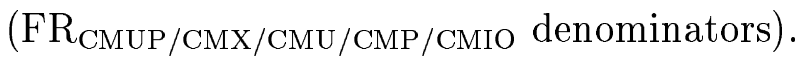
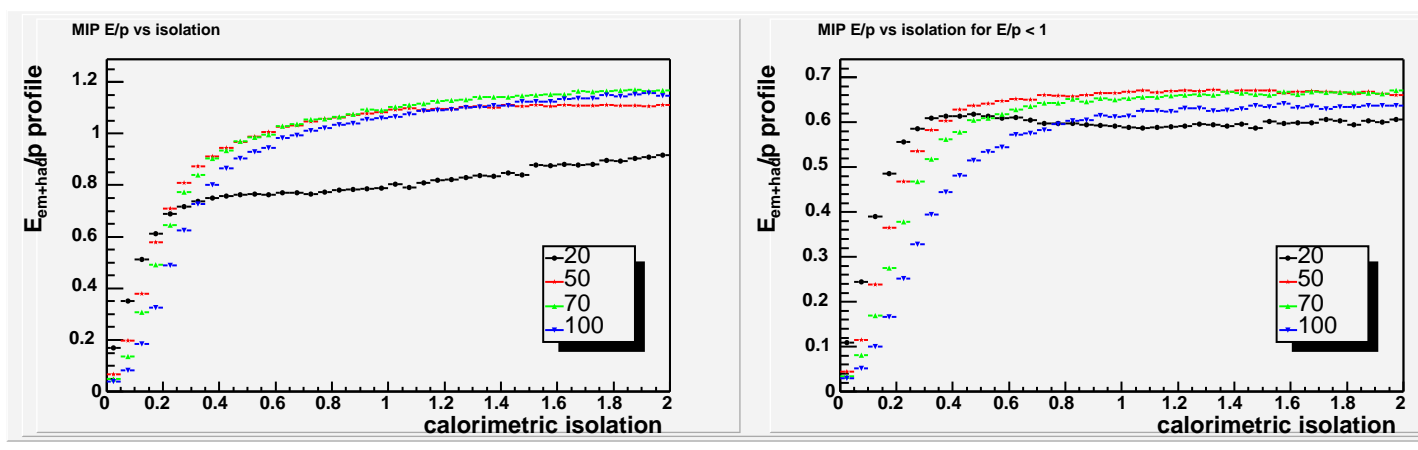

Figure 6.3: $E_{\text {em+had }} / p$ profiles vs. cal isoFrac of MIP tracks in the jet samples before (left) and after (right) requiring $E_{e m+h a d} / p<1$. 

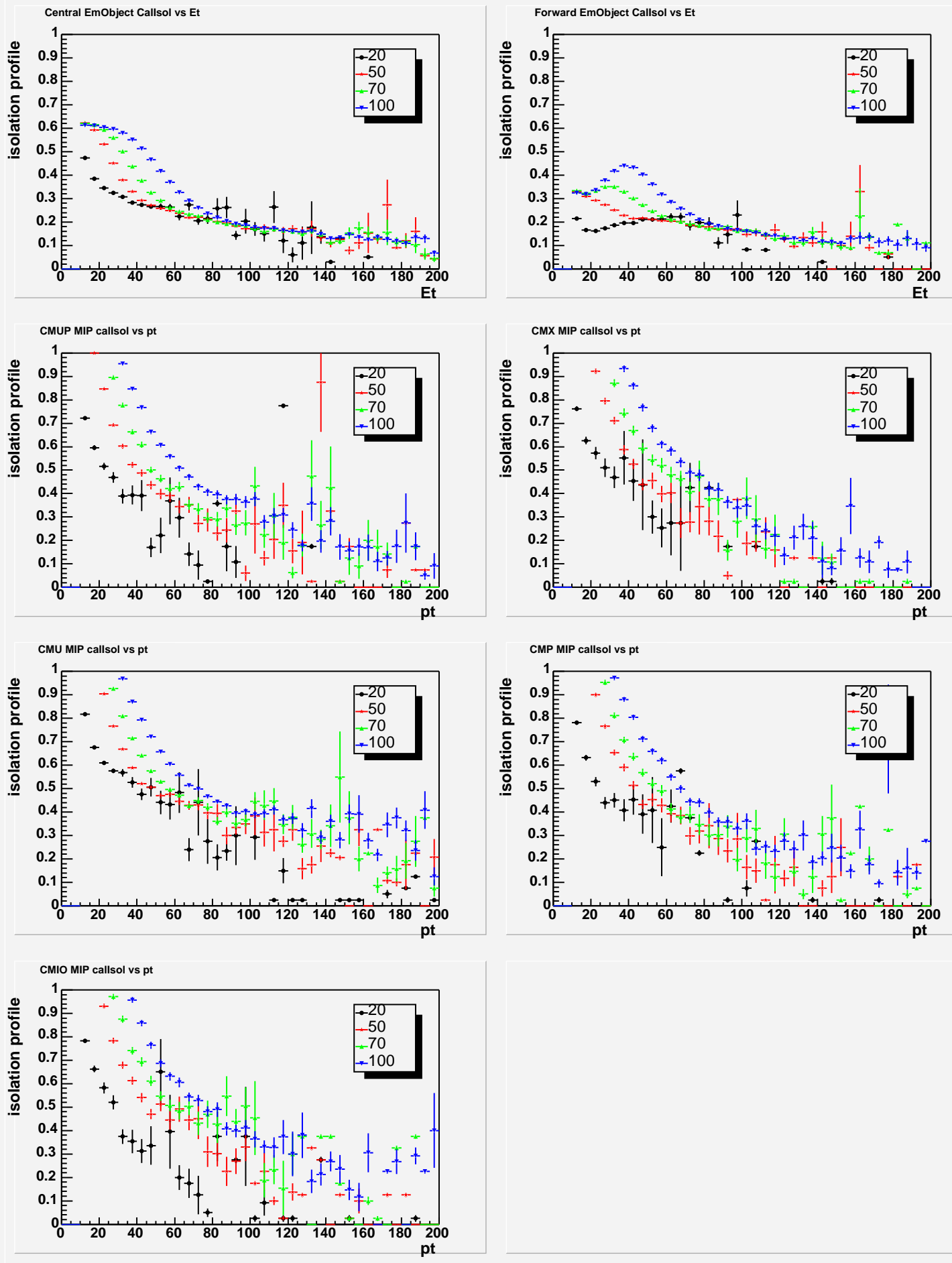

Figure 6.4: Cal isoFrac profiles vs. $E_{t}$ of fakeable objects in the jet samples. 

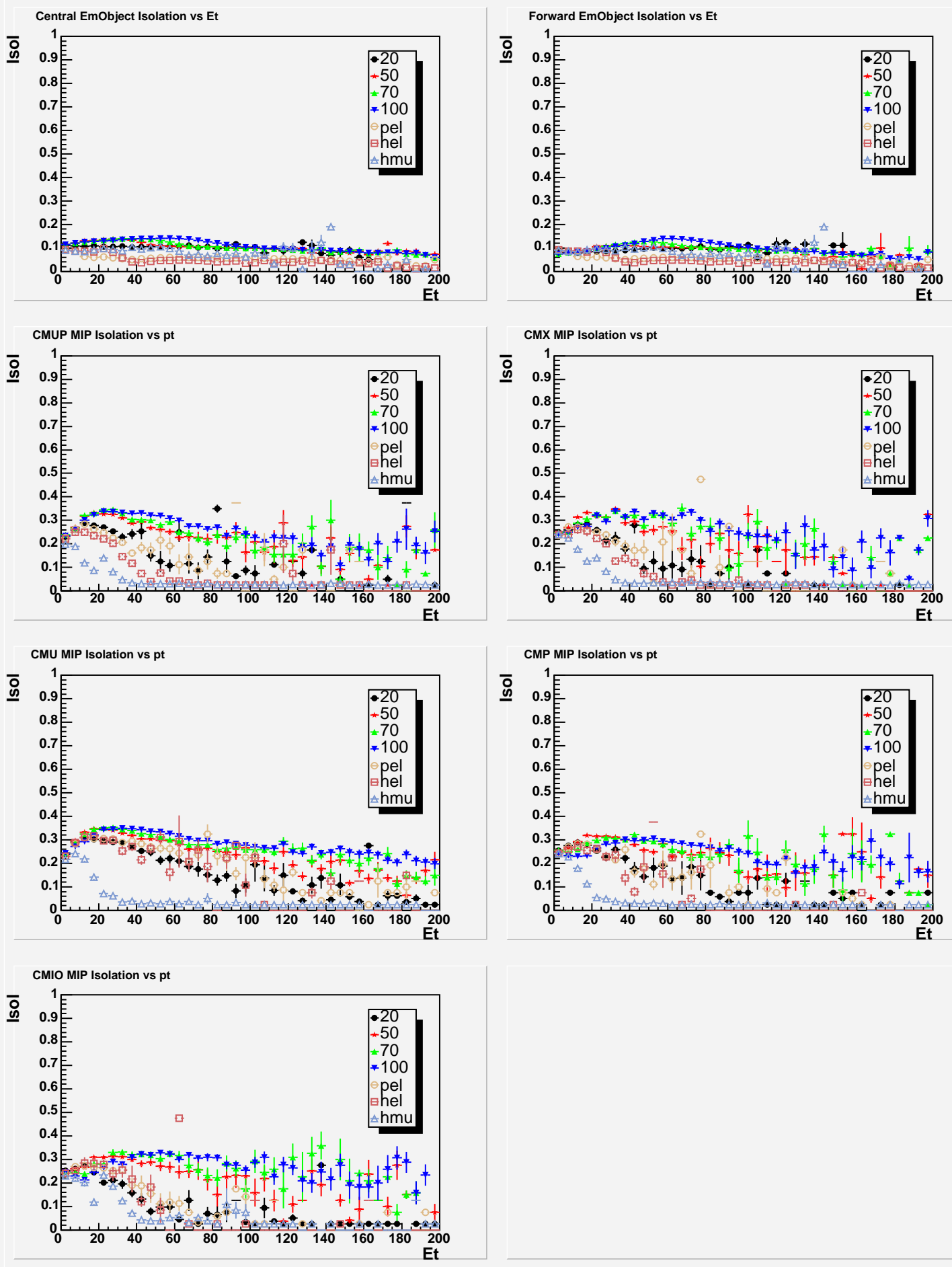

Figure 6.5: Cal isoFrac profiles vs. $E_{t}$ of fakeable objects with cal isoFrac $<0.2$ in the high $p_{t}$ lepton and jet samples. 

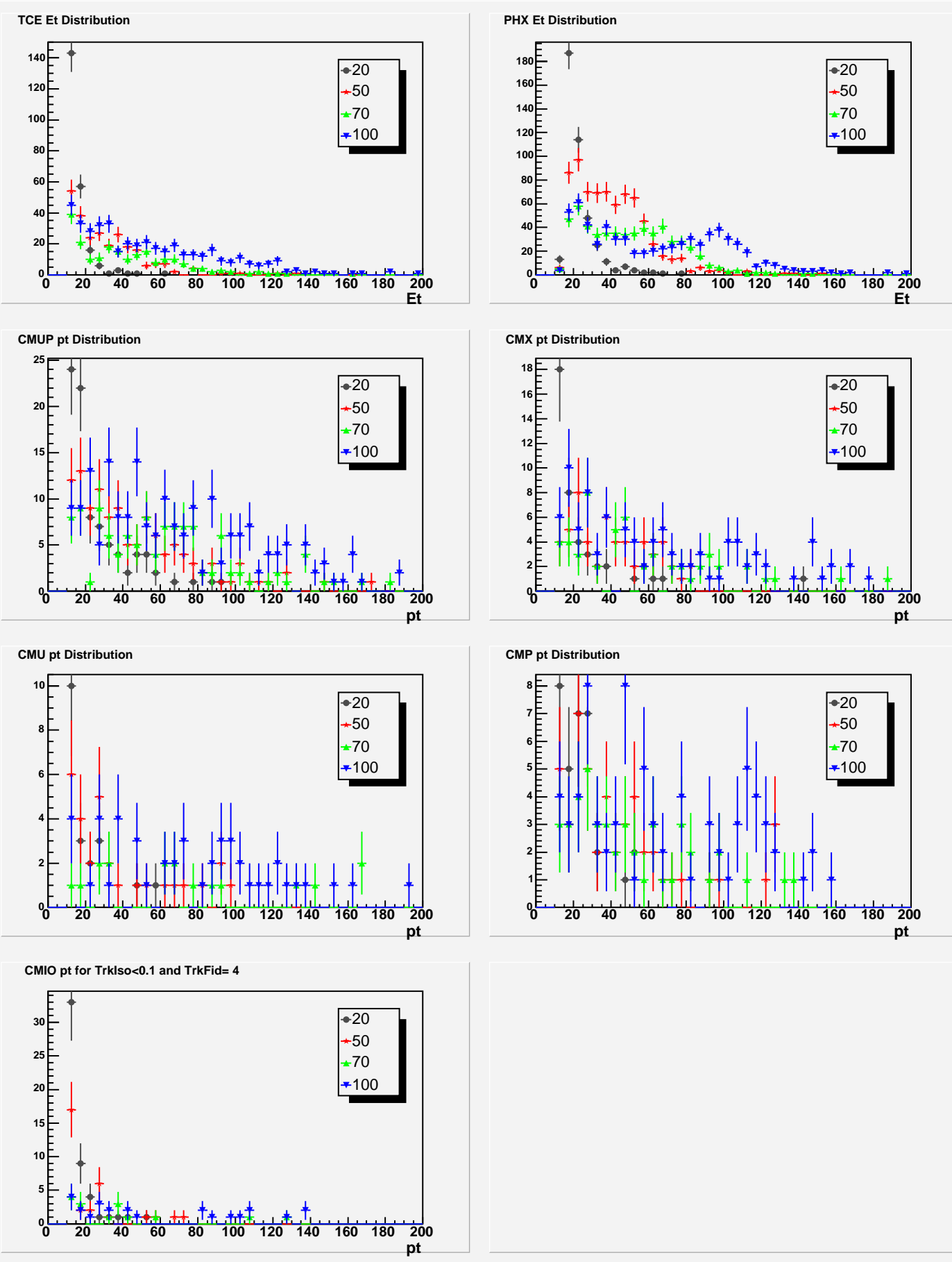

Figure 6.6: $E_{t}$ distributions of leptons in the jet samples. 
Central emObject Et Distribution

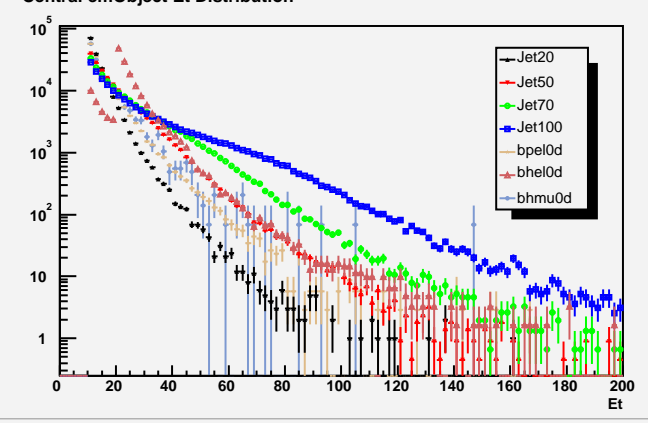

CMUP MIP pt Distribution

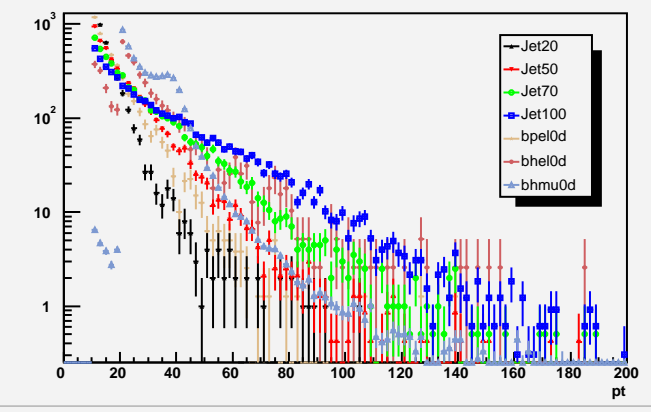

CMU MIP pt Distribution
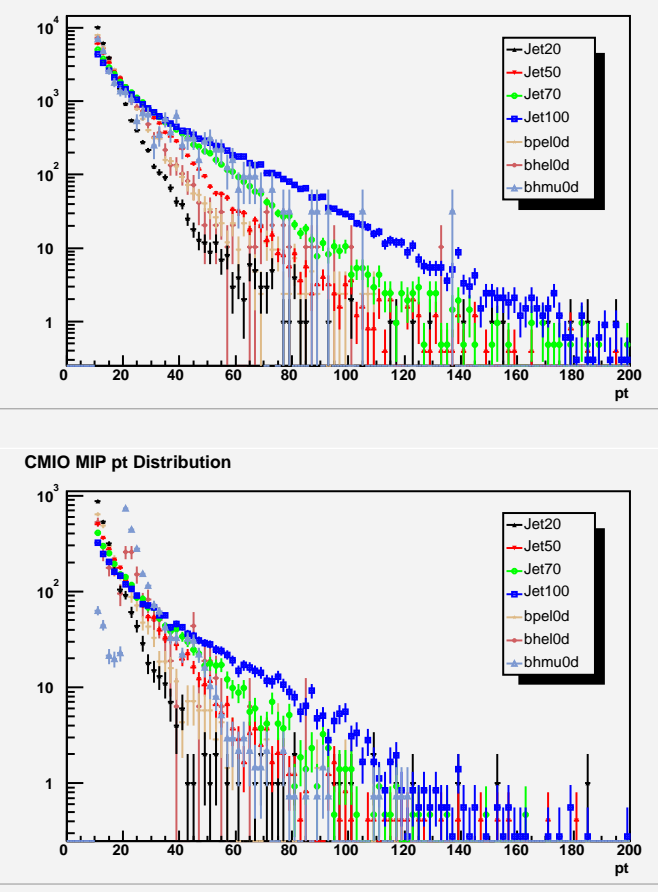

Plug emObject Et Distribution

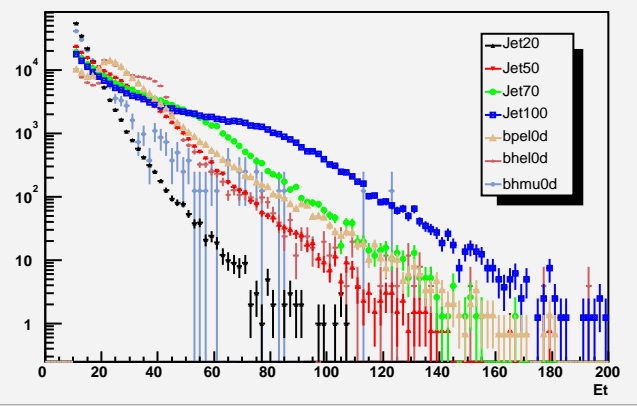

CMX MIP pt Distribution

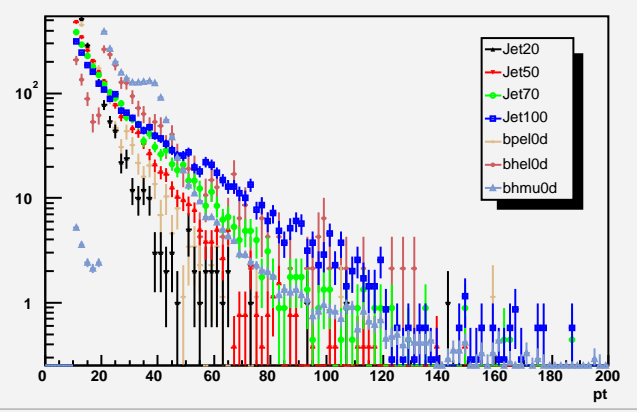

CMP MIP pt Distribution

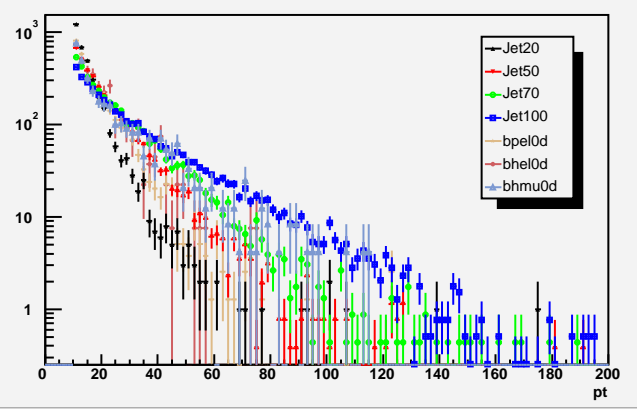

Figure 6.7: Normalized $E_{t}$ distributions of fakeable objects in the high $p_{t}$ lepton and jet samples. 

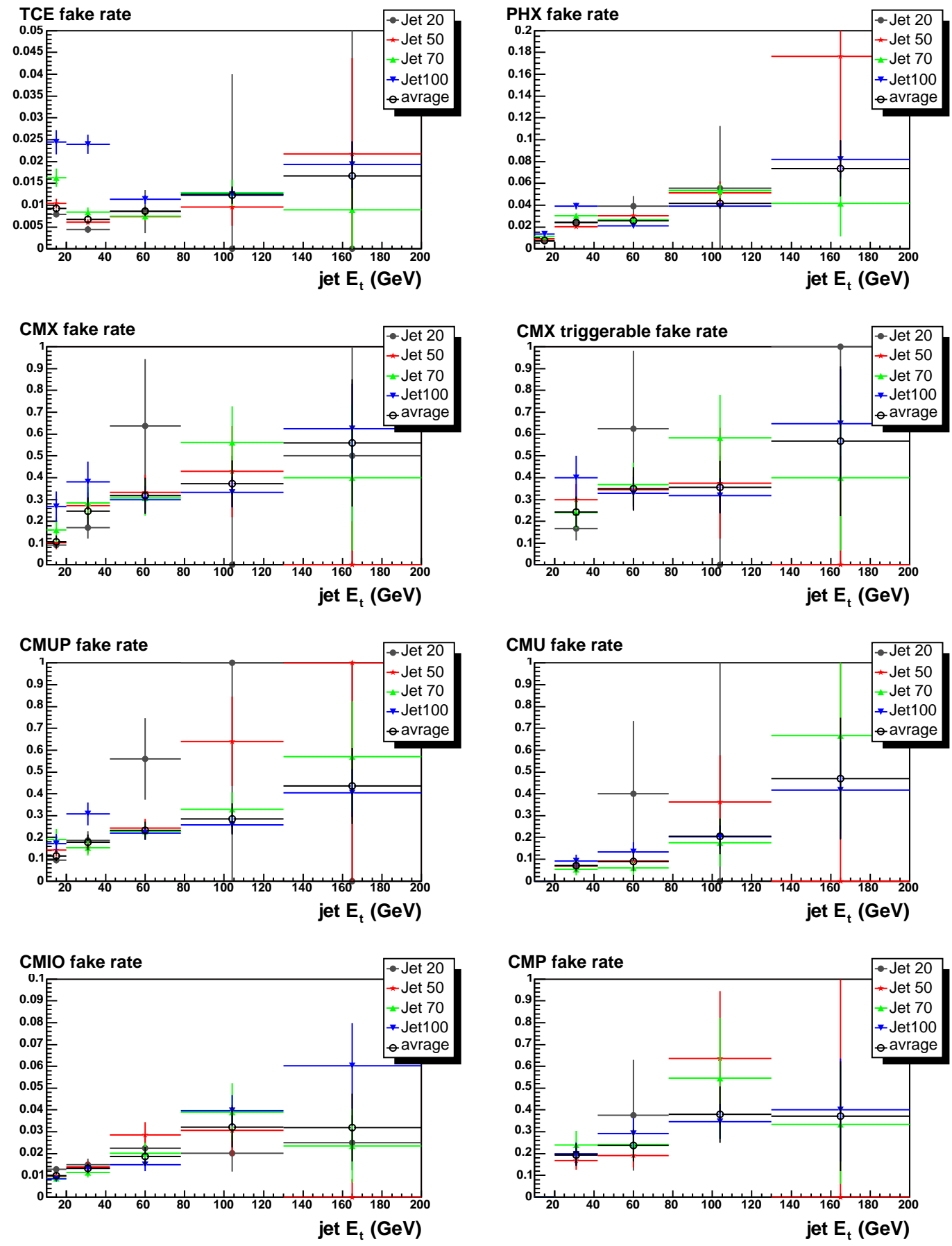

Figure 6.8: Lepton fake rates estimated using rebinned Figure 6.6 and Figure 6.7, including the averages over the analyzed jet samples. 


\begin{tabular}{|c|c|c|c|c|c|c|c|}
\hline lepton & fakrat & densrc & number predicted & lepton & fakrat & densrc & number predicted \\
\hline TCE & Jet 20 & Jet 20 & $229.00 \pm 15.31$ & PHX & Jet 20 & Jet 20 & $420.00 \pm 20.83$ \\
\hline TCE & Jet 50 & Jet 20 & $301.47 \pm 27.80$ & PHX & Jet 50 & Jet 20 & $468.97 \pm 31.79$ \\
\hline TCE & Jet 70 & Jet 20 & $464.15 \pm 54.10$ & PHX & Jet 70 & Jet 20 & $609.97 \pm 52.36$ \\
\hline TCE & Jet100 & Jet 20 & $762.59 \pm 72.16$ & PHX & Jet100 & Jet 20 & $739.02 \pm 59.08$ \\
\hline TCE & Jet 20 & Jet 50 & $204.25 \pm 45.64$ & $\overline{\mathrm{PHX}}$ & Jet 20 & Jet 50 & $847.53 \pm 91.53$ \\
\hline TCE & Jet 50 & Jet 50 & $253.00 \pm 16.03$ & PHX & Jet 50 & Jet 50 & $728.00 \pm 27.63$ \\
\hline TCE & Jet 70 & Jet 50 & $344.55 \pm 27.31$ & PHX & Jet 70 & Jet 50 & $876.33 \pm 43.61$ \\
\hline TCE & Jet100 & Jet 50 & $707.25 \pm 46.04$ & PHX & Jet100 & Jet 50 & $990.82 \pm 53.90$ \\
\hline TCE & Jet 20 & Jet 70 & $142.63 \pm 82.99$ & PHX & Jet 20 & Jet 70 & $628.15 \pm 115.99$ \\
\hline TCE & Jet 50 & Jet 70 & $168.22 \pm 13.37$ & PHX & Jet 50 & Jet 70 & $533.17 \pm 25.07$ \\
\hline TCE & Jet 70 & Jet 70 & $209.00 \pm 14.61$ & PHX & Jet 70 & Jet 70 & $563.00 \pm 24.42$ \\
\hline TCE & Jet100 & Jet 70 & $384.96 \pm 21.36$ & PHX & Jet100 & Jet 70 & $560.09 \pm 25.92$ \\
\hline TCE & Jet 20 & Jet100 & $140.71 \pm 372.10$ & PHX & Jet 20 & Jet100 & $780.98 \pm 349.08$ \\
\hline TCE & Jet 50 & Jet100 & $228.92 \pm 36.93$ & PHX & Jet 50 & Jet100 & $730.38 \pm 73.81$ \\
\hline TCE & Jet 70 & Jet100 & $274.04 \pm 26.45$ & PHX & Jet 70 & Jet100 & $730.41 \pm 44.41$ \\
\hline TCE & Jet100 & Jet100 & $423.00 \pm 20.93$ & PHX & Jet100 & Jet100 & $668.00 \pm 26.71$ \\
\hline leptype & fakrat & densrc & number predicted & lepton & fakrat & densrc & number predicted \\
\hline CMX & Jet 20 & Jet 20 & 51.00 \pm 8.48 & $\overline{\overline{\text { CMXtrg }}}$ & Jet 20 & Jet 20 & $1717.00 \pm 5.47$ \\
\hline CMX & Jet 50 & Jet 20 & $59.61 \pm 11.58$ & $\mathrm{CMX}_{\operatorname{trg}}$ & Jet 50 & Jet 20 & $23.22 \pm 5.49$ \\
\hline CMX & Jet 70 & Jet 20 & $80.00 \pm 19.03$ & $\mathrm{CMX}_{\operatorname{trg}}$ & Jet 70 & Jet 20 & $20.39 \pm 5.55$ \\
\hline CMX & Jet100 & Jet 20 & $121.82 \pm 24.08$ & $\mathrm{CMX}_{\operatorname{trg}}$ & Jet100 & Jet 20 & $30.32 \pm 7.54$ \\
\hline CMX & Jet 20 & Jet 50 & $75.86 \pm 24.73$ & CMXtrg & Jet 20 & Jet 50 & $51.08 \pm 22.26$ \\
\hline CMX & Jet 50 & Jet 50 & $69.00 \pm 10.41$ & CMXtrg & Jet 50 & Jet 50 & $46.00 \pm 8.76$ \\
\hline CMX & Jet 70 & Jet 50 & $78.89 \pm 12.62$ & $\mathrm{CMX}_{\operatorname{trg}}$ & Jet 70 & Jet 50 & $45.31 \pm 9.25$ \\
\hline CMX & Jet100 & Jet 50 & $98.42 \pm 14.22$ & $\mathrm{CMX}_{\operatorname{trg}}$ & Jet100 & Jet 50 & $53.72 \pm 10.03$ \\
\hline CMX & Jet 20 & Jet 70 & $59.03 \pm 25.89$ & $\mathrm{CMX}_{\operatorname{trg}}$ & Jet 20 & Jet 70 & $44.62 \pm 23.09$ \\
\hline CMX & Jet 50 & Jet 70 & $59.01 \pm 10.36$ & $\mathrm{CMX}_{\operatorname{trg}}$ & Jet 50 & Jet 70 & $42.03 \pm 9.55$ \\
\hline CMX & Jet 70 & Jet 70 & $68.00 \pm 10.75$ & $\mathrm{CMX}_{\operatorname{trg}}$ & Jet 70 & Jet 70 & $47.00 \pm 9.19$ \\
\hline CMX & Jet100 & Jet 70 & $74.51 \pm 10.11$ & CMXtrg & Jet100 & Jet 70 & $48.61 \pm 8.19$ \\
\hline CMX & Jet 20 & Jet100 & $92.85 \pm 56.99$ & CMXtrg & Jet 20 & Jet100 & $73.67 \pm 50.58$ \\
\hline CMX & Jet 50 & Jet100 & $96.37 \pm 22.16$ & $\mathrm{CMX}_{\operatorname{trg}}$ & Jet 50 & Jet100 & $68.51 \pm 21.54$ \\
\hline CMX & Jet 70 & Jet100 & $120.19 \pm 20.90$ & $\mathrm{CMX}_{\operatorname{trg}}$ & Jet 70 & Jet100 & $88.21 \pm 18.20$ \\
\hline CMX & Jet100 & Jet100 & $118.00 \pm 14.25$ & $\mathrm{CMX}_{\operatorname{trg}}$ & Jet100 & Jet100 & $80.00 \pm 11.93$ \\
\hline
\end{tabular}

Table 6.7: Number of fake TCE/PHX/CMX/CMXtriggerable leptons, predicted with Jet20/50/70/100 fake rate applied to the denominators in source jet samples, showing overall agreement. The true numbers of leptons in the densrc jet sample are retained when the lepton fake rates from the same jet sample are applied. 


\begin{tabular}{|c|c|c|c|c|c|c|c|}
\hline lepton & fakrat & densrc & number predicted & lepton & fakrat & densrc & number predicted \\
\hline CMUP & Jet 20 & Jet 20 & $86.00 \pm 11.15$ & $\mathrm{CMU}$ & Jet 20 & Jet 20 & $7.00 \pm 3.21$ \\
\hline CMUP & Jet 50 & Jet 20 & $96.23 \pm 15.06$ & $\mathrm{CMU}$ & Jet 50 & Jet 20 & $5.69 \pm 1.77$ \\
\hline CMUP & Jet 70 & Jet 20 & $117.64 \pm 24.31$ & $\mathrm{CMU}$ & Jet 70 & Jet 20 & $4.37 \pm 2.07$ \\
\hline CMUP & Jet100 & Jet 20 & $128.45 \pm 22.70$ & $\mathrm{CMU}$ & Jet100 & Jet 20 & $7.39 \pm 2.33$ \\
\hline$\overline{\text { CMUP }}$ & Jet 20 & Jet 50 & $177.45 \pm 41.10$ & $\overline{\mathrm{CMU}}$ & Jet 20 & Jet 50 & $31.88 \pm 21.90$ \\
\hline CMUP & Jet 50 & Jet 50 & $121.00 \pm 13.53$ & $\mathrm{CMU}$ & Jet 50 & Jet 50 & $19.00 \pm 5.02$ \\
\hline CMUP & Jet 70 & Jet 50 & $119.28 \pm 15.10$ & $\mathrm{CMU}$ & Jet 70 & Jet 50 & $13.88 \pm 4.78$ \\
\hline CMUP & Jet100 & Jet 50 & $151.03 \pm 17.45$ & $\mathrm{CMU}$ & Jet100 & Jet 50 & $23.28 \pm 5.31$ \\
\hline CMUP & Jet 20 & Jet 70 & $217.71 \pm 81.82$ & $\overline{\mathrm{CMU}}$ & Jet 20 & Jet 70 & $31.54 \pm 28.17$ \\
\hline CMUP & Jet 50 & Jet 70 & $142.10 \pm 23.67$ & $\mathrm{CMU}$ & Jet 50 & Jet 70 & $17.29 \pm 7.94$ \\
\hline CMUP & Jet 70 & Jet 70 & $116.00 \pm 13.21$ & $\mathrm{CMU}$ & Jet 70 & Jet 70 & $15.00 \pm 4.72$ \\
\hline CMUP & Jet100 & Jet 70 & $126.59 \pm 12.35$ & $\mathrm{CMU}$ & Jet 100 & Jet 70 & $21.49 \pm 4.37$ \\
\hline CMUP & Jet 20 & Jet100 & $388.87 \pm 213.23$ & $\overline{\mathrm{CMU}}$ & Jet 20 & Jet100 & $40.48 \pm 88.52$ \\
\hline CMUP & Jet 50 & Jet100 & $277.95 \pm 64.95$ & $\mathrm{CMU}$ & Jet 50 & Jet100 & $45.60 \pm 22.20$ \\
\hline CMUP & Jet 70 & Jet100 & $196.43 \pm 24.61$ & $\mathrm{CMU}$ & Jet 70 & Jet100 & $33.77 \pm 11.72$ \\
\hline CMUP & Jet100 & Jet100 & $193.00 \pm 17.18$ & $\mathrm{CMU}$ & Jet100 & Jet100 & $43.00 \pm 7.67$ \\
\hline lepton & fakrat & densrc & number predicted & lepton & fakrat & densrc & number predicted \\
\hline$\overline{\mathrm{CMIO}}$ & Jet 20 & Jet 20 & 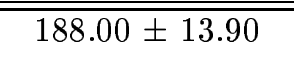 & 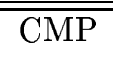 & Jet 20 & Jet 20 & $19.00 \pm 5.26$ \\
\hline $\mathrm{CMIO}$ & Jet 50 & Jet 20 & $154.54 \pm 15.34$ & CMP & Jet 50 & Jet 20 & $14.99 \pm 3.73$ \\
\hline $\mathrm{CMIO}$ & Jet 70 & Jet 20 & $139.06 \pm 16.14$ & CMP & Jet 70 & Jet 20 & $21.09 \pm 5.67$ \\
\hline CMIO & Jet100 & Jet 20 & $144.53 \pm 12.36$ & CMP & Jet100 & Jet 20 & $18.17 \pm 4.56$ \\
\hline $\mathrm{CMIO}$ & Jet 20 & Jet 50 & $140.80 \pm 11.56$ & CMP & Jet 20 & Jet 50 & $49.98 \pm 19.91$ \\
\hline $\mathrm{CMIO}$ & Jet 50 & Jet 50 & $124.00 \pm 11.35$ & CMP & Jet 50 & Jet 50 & $40.00 \pm 7.85$ \\
\hline $\mathrm{CMIO}$ & Jet 70 & Jet 50 & $108.23 \pm 11.34$ & CMP & Jet 70 & Jet 50 & $51.01 \pm 10.22$ \\
\hline CMIO & Jet100 & Jet 50 & $112.52 \pm 8.49$ & CMP & Jet100 & Jet 50 & $47.94 \pm 8.42$ \\
\hline $\mathrm{CMIO}$ & Jet 20 & Jet 70 & $121.05 \pm 10.52$ & $\overline{\mathrm{CMP}}$ & Jet 20 & Jet 70 & $35.95 \pm 15.59$ \\
\hline $\mathrm{CMIO}$ & Jet 50 & Jet 70 & $110.05 \pm 10.18$ & CMP & Jet 50 & Jet 70 & $30.06 \pm 8.68$ \\
\hline $\mathrm{CMIO}$ & Jet 70 & Jet 70 & $95.00 \pm 9.89$ & CMP & Jet 70 & Jet 70 & $39.00 \pm 7.86$ \\
\hline CMIO & Jet100 & Jet 70 & $97.01 \pm 7.20$ & CMP & Jet100 & Jet 70 & $37.24 \pm 6.44$ \\
\hline $\mathrm{CMIO}$ & Jet 20 & Jet100 & $233.43 \pm 21.29$ & $\overline{\mathrm{CMP}}$ & Jet 20 & Jet100 & $48.95 \pm 21.76$ \\
\hline $\mathrm{CMIO}$ & Jet 50 & Jet100 & $218.69 \pm 20.88$ & CMP & Jet 50 & Jet100 & $76.85 \pm 25.71$ \\
\hline $\mathrm{CMIO}$ & Jet 70 & Jet100 & $193.02 \pm 20.72$ & CMP & Jet 70 & Jet100 & $84.19 \pm 22.51$ \\
\hline $\mathrm{CMIO}$ & Jet100 & Jet100 & $199.00 \pm 14.36$ & CMP & Jet 100 & Jet100 & $71.00 \pm 10.62$ \\
\hline
\end{tabular}

Table 6.8: Number of CMUP/CMU/CMP/CMIO leptons, predicted with Jet20/50/70/100 fake rate applied to the denominators in source jet samples, showing overall agreement. The true numbers of leptons in the densrc jet sample are retained when the lepton fake rates from the same jet sample are applied. 


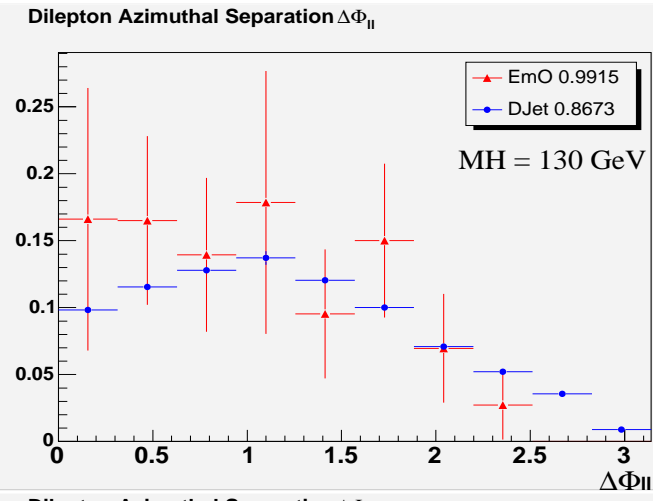

Dilepton Azimuthal Separation $\Delta \Phi_{\text {II }}$

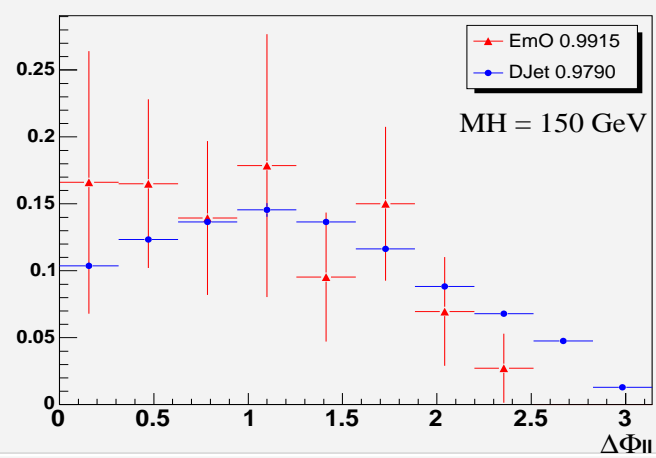

Dilepton Azimuthal Separation $\Delta \Phi_{\|}$

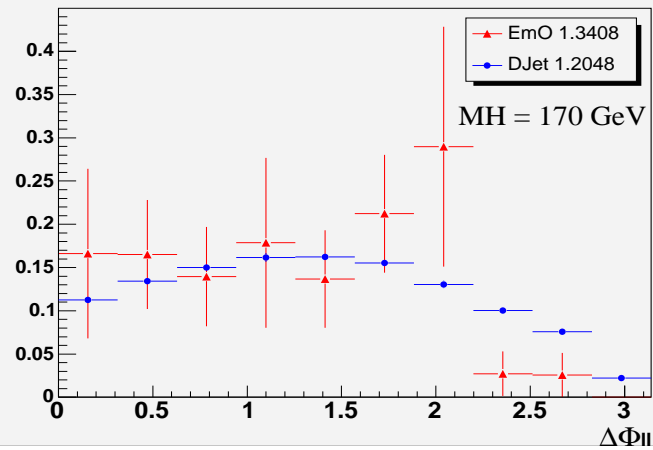

Dilepton Azimuthal Separation $\Delta \Phi_{\|}$

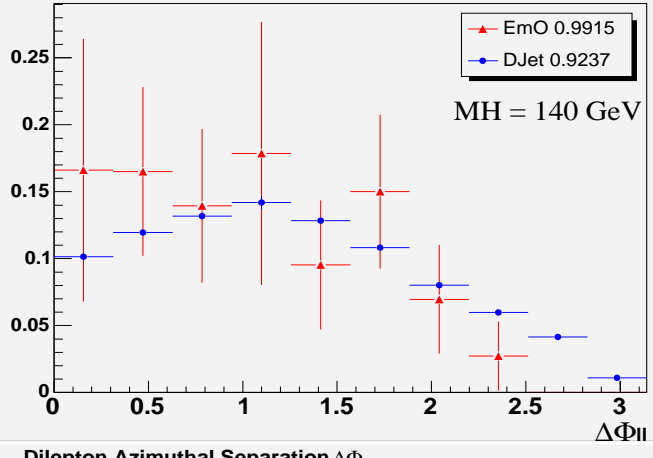

Dilepton Azimuthal Separation $\Delta \Phi_{\|}$

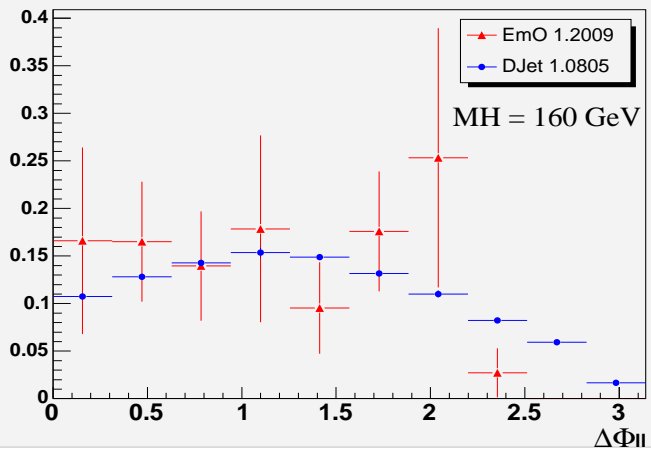

Dilepton Azimuthal Separation $\Delta \Phi_{\|}$

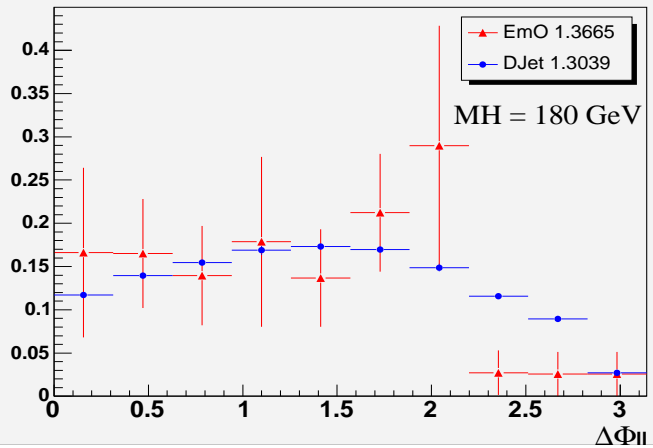

Figure 6.9: Dilepton azimuthal angular separation $\Delta \phi_{\ell \ell}$ distributions of jet fake background estimated with either electromagnetic objects (EmO) or generic jets (DJet) as electron fake rate denominators and minimum ionizing particles (MIP) as muon fake rate denominators, showing reasonably good shape agreement although EmO carry much larger errors than DJet due to much smaller statistics (and CPU consumption). The numbers in legends are histogram integrals. 
For the estimate of fake background in the $H W W$ dilepton search we used the statistics-weighted average lepton fake rates and took the largest difference among the jet samples as systematic uncertainty.

Good agreement was found among the analyzed jet data samples in the estimated lepton fake rates, in the strong $p_{t}$ dependence of them and in the cross-sample predicted numbers of fake leptons.

\subsubsection{Lepton Fake Background Estimate}

Lepton selection cuts are listed in Table 6.2, Table 6.4 and Table 6.5. Denominator fakeable object selection cuts are listed in Table 6.3 and Table 6.6. Event selection cuts are available in Chapter 7.

Double fake background, with both leptons faked by denominator objects, was included but did not have any significance because the lepton fake rates in most lepton-populated $p_{t}$ region were $10^{-1}-10^{-2}$.

The estimated QCD/ $W+$ jet fake background is included in all applicable plots in the following chapters. The estimated fake background together with MC-predicted background expectation agreed with observation.

We performed an interesting cross-check by changing the electron fake rate denominators from electromagnetic objects to generic jets $(|\eta|<1.1$ for TCE and $1.2<|\eta|<2.0$ for PHX; half and half charge sign probabilities) and we found the change in fake background integral numbers to be within $15 \%$. The shapes can be found in Figure 6.9.

As shown in Table 6.7 and Table 6.8 and Figure 6.8 and Figure 6.9, the agreement in our lepton fake estimate was found to be very good. Nevertheless we quote $50 \%$ 
uncertainty from sample dependence on the estimated fake background, just to be conservative and consistent with other CDF lepton fake estimates. 


\section{Chapter 7}

\section{Event Selection}

Event selection is used to optimize the ratio of signal to background, $S / \sqrt{B}$, and hence improve the sensitivity of analysis. With event selection, we may select events that have signal features or to reject events that have background features. Often the selection cuts or cut thresholds are adjusted to balance between signal acceptance and $\mathrm{S} / \sqrt{\mathrm{B}}$ for the best results. Given the known Higgs sensitivity at the Tevatron [28], this analysis was performed with the expectation of observing no signal excess and extracting the upper limits on the $H W W$ production cross-section.

\subsection{Search Strategy}

In search for the $H W W$ signal, we used the $W W$ dilepton decay channel, $W^{+} W^{-} \rightarrow \ell^{+} \nu \ell^{-} \bar{\nu}$, where each of the leptons $\ell$ can be an electron, a muon or a leptonically decayed tau lepton. Branching fractions of the tau leptonic decays are given in Table 1.2. Branching fractions of the $W$ boson decays are given in Table 1.5. The $W W$ dilepton decay branching fraction is only $6.4 \%$ but this channel is almost

free of QCD background. Due to the absence of QCD background, the $S / \sqrt{B}$ of the $W W$ dilepton decay channel is much higher than that of any hadronic $W W$ decay 


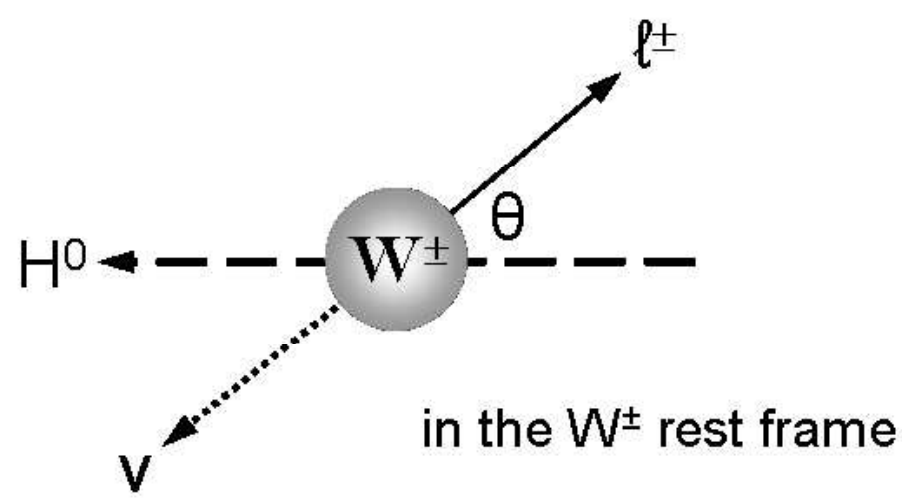

Figure 7.1: Framework for the $H \rightarrow W^{+} W^{-} \rightarrow \ell^{+} \bar{\nu}_{\ell} \ell^{-} \nu_{\ell}$ angular distributions.

channel; therefore, the sensitivity of an analysis in this channel is higher although the signal acceptance is apparently lower.

In discriminating the Higgs signal from the background, the Higgs boson's spin-0 property plays an important role, the spin-1 $W^{ \pm}$bosons from the decay of a single SM Higgs boson $H^{(0)}$ fly back-to-back in the Higgs boson's rest frame with opposite momenta and equal helicities. The proportions of left-handed $(L)$, longitudinal $(\vdash)$ and right-handed $(R) W$ bosons are each $\frac{1}{3}$.

The amplitudes of $W \rightarrow \ell \nu$ decays for given helicities are

$$
\begin{gathered}
\left|\mathcal{M}\left(W^{+}{ }_{L}\right)\right|^{2}=\left|\mathcal{M}\left(W^{-}{ }_{R}\right)\right|^{2}=(1-\cos \theta)^{2} \\
\left|\mathcal{M}\left(W^{+}{ }_{\vdash}\right)\right|^{2}=\left|\mathcal{M}\left(W^{-}{ }_{\vdash}\right)\right|^{2}=1-\cos ^{2} \theta \\
\left|\mathcal{M}\left(W^{+}{ }_{R}\right)\right|^{2}=\left|\mathcal{M}\left(W^{-}{ }_{L}\right)\right|^{2}=(1+\cos \theta)^{2}
\end{gathered}
$$

where $\theta_{\left(\ell^{ \pm}, W^{ \pm}\right)}$is the angle between the charged lepton's momentum in its mother $W$ boson's rest frame and the boost direction from the Higgs boson's rest frame to the mother $W$ boson's rest frame, as shown in Figure 7.1. 
For $H \rightarrow W^{+} W^{-} \rightarrow \ell^{+} \bar{\nu}_{\ell} \ell^{-} \nu_{\ell}$, where the $W^{+}$and $W^{-}$bosons fly back-to-back with the same helicities,

$$
\begin{gathered}
\left|\mathcal{M}\left(W_{L}^{+}{ }_{L}\right)\right|^{2}=\left(1-\cos \theta_{\ell^{+}, W^{+}}\right)^{2}=\left[1-\cos \left(\pi-\theta_{\ell^{+}, W^{-}}\right)\right]^{2}=\left(1+\cos \theta_{\ell^{+}, W^{-}}\right)^{2} \\
\left|\mathcal{M}\left(W^{-}{ }_{L}\right)\right|^{2}=\left(1+\cos \theta_{\ell^{-}, W^{-}}\right)^{2}=\left[1+\cos \left(\pi-\theta_{\ell^{-}, W^{+}}\right)\right]^{2}=\left(1-\cos \theta_{\ell^{-}, W^{+}}\right)^{2} \\
\left|\mathcal{M}\left(W^{+}+\right)\right|^{2}=1-\cos ^{2} \theta_{\ell^{+}, W^{+}}=1-\cos ^{2}\left(\pi-\theta_{\ell^{+}, W^{-}}\right)=1-\cos ^{2} \theta_{\ell^{+}, W^{-}} \\
\left|\mathcal{M}\left(W^{-}{ }^{-}\right)\right|^{2}=1-\cos ^{2} \theta_{\ell^{-}, W^{-}}=1-\cos ^{2}\left(\pi-\theta_{\ell^{-}, W^{+}}\right)=1-\cos ^{2} \theta_{\ell^{-}, W^{+}} \\
\left|\mathcal{M}\left(W^{+}{ }_{R}\right)\right|^{2}=\left(1+\cos \theta_{\ell^{+}, W^{+}}\right)^{2}=\left[1+\cos \left(\pi-\theta_{\ell^{+}, W^{-}}\right)\right]^{2}=\left(1-\cos \theta_{\ell^{+}, W^{-}}\right)^{2} \\
\left|\mathcal{M}\left(W^{-}{ }_{R}\right)\right|^{2}=\left(1-\cos \theta_{\ell^{-}, W^{-}}\right)^{2}=\left[1-\cos \left(\pi-\theta_{\ell^{-}, W^{+}}\right)\right]^{2}=\left(1+\cos \theta_{\ell^{-}, W^{+}}\right)^{2}
\end{gathered}
$$

there is a transition of $\theta_{\ell^{ \pm}, W^{ \pm}} \rightarrow \theta_{\ell^{ \pm}, W^{\mp}}=\pi-\theta_{\ell^{ \pm}, W^{ \pm}}$when the angle is viewed from the other (the charged lepton's aunt) $W$ boson's rest frame.

The charged leptons from a $H W W$ dilepton decay have the same amplitude distributions in the Higgs boson's rest frame and hence have a tendency to fly parallel to each other. The neutrinos also tend to fly parallel, since the charged lepton and the neutrino from each $W \rightarrow \ell \nu$ decay fly back-to-back in the $W$ boson's rest frame.

In $p \bar{p}$ collisions at the Tevatron, where the longitudinal $E_{c m}$ varies but the transverse $E_{c m}=0$, the tendency of $H W W$ dilepton flying parallel has a few observable effects that can be exploited in a search for the Higgs boson, with the most relevant for discriminating the signal from the background being a small angular separation between the two leptons in the transverse plane $\Delta \phi_{\ell \ell}$.

Our selection strategy was therefore set to

1. select events for the best $S / \sqrt{B}$ within a reasonable range of signal acceptance; 
2. adjust the cut thresholds as needed for the best expected limits on signal crosssection, where the expected limits are extracted using the predicted signal and background $\Delta \phi_{\ell \ell}$ distributions.

\subsection{Leptons}

Events with exactly two identified leptons were selected.

\subsubsection{Flavors}

Lepton flavors were restricted to electron and muon, for which the identification criteria are described in Chapter 5, because electrons and muons are stable final states and can be reliably identified in contrast to taus. Nevertheless, leptonic tau decays, $\tau \rightarrow \ell \nu_{\ell} \nu_{\tau}$ where $\ell \in\{e, \mu\}$, were implicitly included.

\subsubsection{Transverse Energies}

The triggering lepton was required to have transverse energy $E_{t}>20 \mathrm{GeV}$ and the other lepton was required to have transverse energy $E_{t}>10 \mathrm{GeV}$ after the following considerations:

1. as the MC study indicates in Figure 7.2, most of the $H W W$ leading leptons have $E_{t}>20 \mathrm{GeV}$ but a significant number of the subleading leptons from off-shell $W$ boson decays have $E_{t}<20 \mathrm{GeV}$;

2. lowering any of the lepton $E_{t}$ thresholds would raise the QCD and $b$-related background;

3. signal acceptance gain motivated us to consider not requiring both leptons to 
have $E_{t}>20 \mathrm{GeV}$ even though that way the analysis would be easier. There would not be much to gain in lowering the leading lepton $E_{t}$ threshold from $20 \mathrm{GeV}$. However, it was worthwhile to lower the subleading lepton $E_{t}$ threshold to $10 \mathrm{GeV}$ as it increased the $H W W$ signal acceptance greatly but raised the $b$ background only slightly and the QCD background moderately. As shown in Table 7.3, the $H W W$ signal acceptance increases $28 \%-510 \%$ depending on the Higgs mass when lepton $E_{t}$ thresholds are lowered from $20 \mathrm{GeV}$ for both leptons to $20 \mathrm{GeV}$ for the triggering leading lepton and $10 \mathrm{GeV}$ for the subleading lepton. The $H W W$ signal acceptance would increase only $1 \%-7 \%$ if the triggers were changed and the lepton $E_{t}$ thresholds were furthered lowered to $10 \mathrm{GeV}$ for both leptons but the QCD and $b$ background would increase significantly;

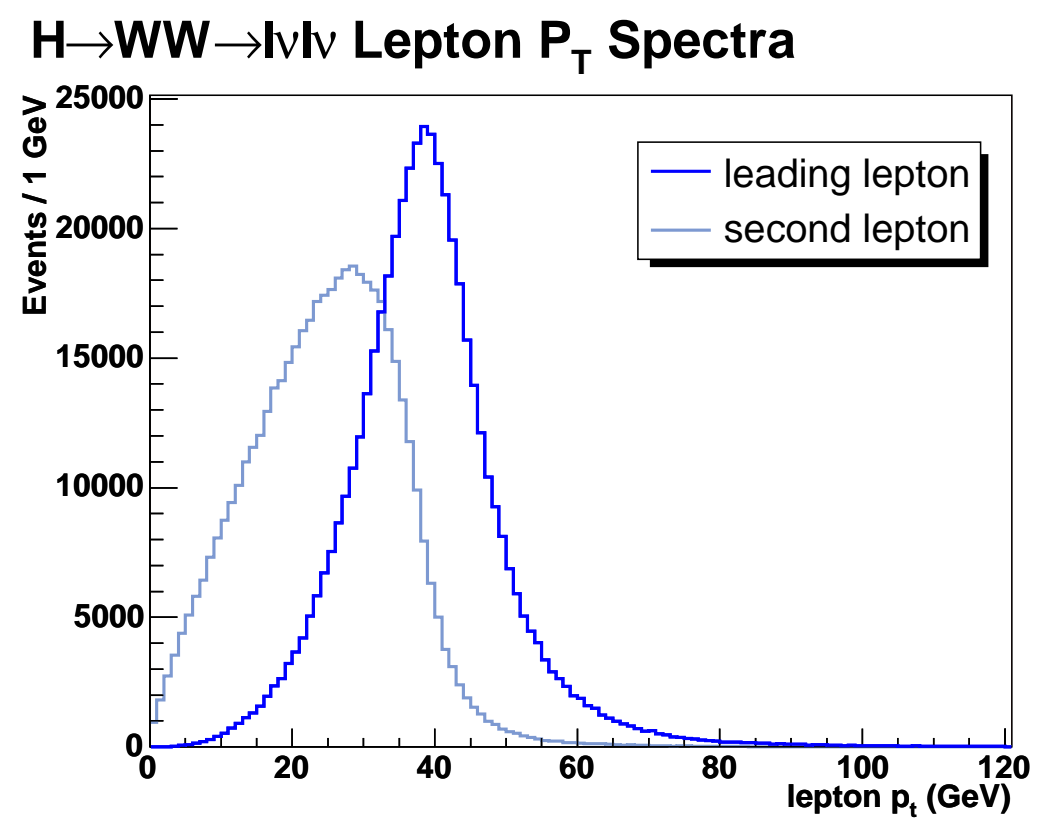

Figure 7.2: $H W W$ leading and subleading (second) lepton $E_{t}$ distributions at the PYTHIA HEPG level for $m_{H}=160 \mathrm{GeV}$. 
HWWIvlv Acceptance (\%) for $M_{H}=110 \mathrm{GeV}$

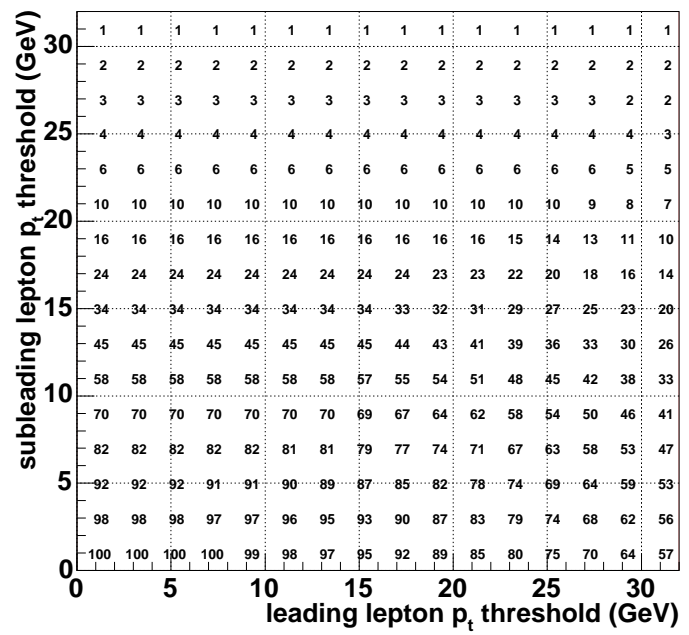

HWWIvlv Acceptance (\%) for $M_{H}=170 \mathrm{GeV}$

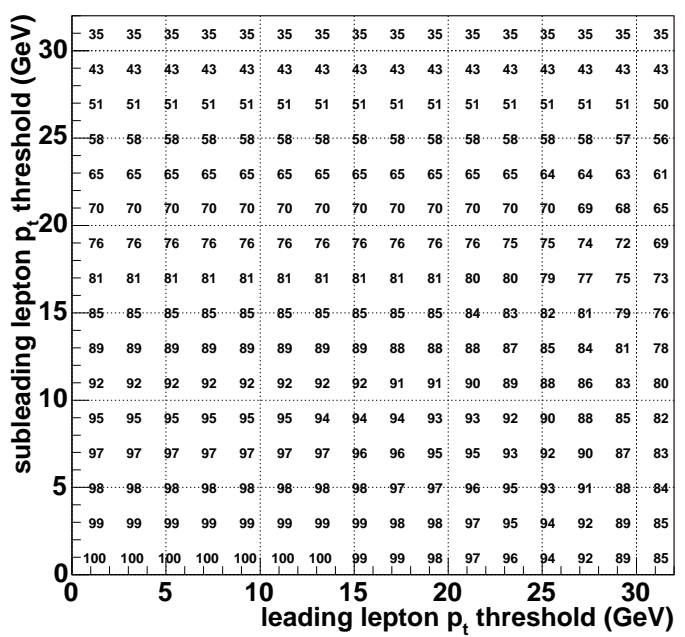

HWWIvlv Acceptance (\%) for $M_{H}=140 \mathrm{GeV}$

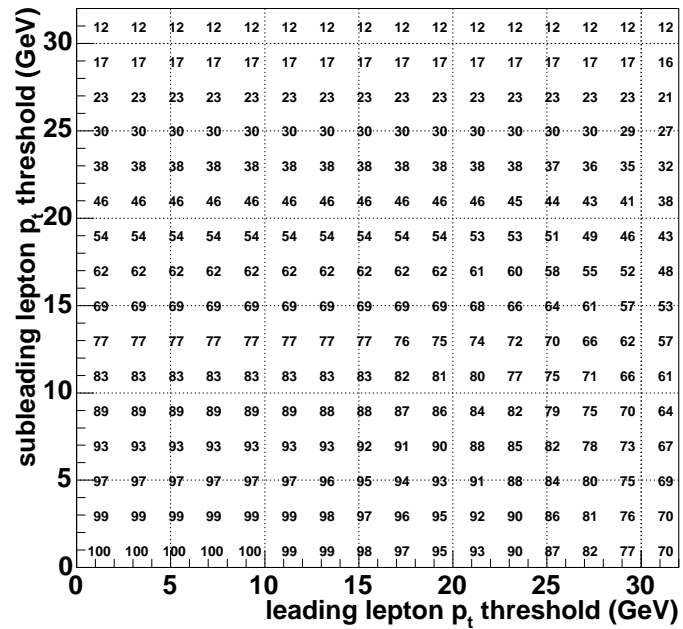

HWWIvlv Acceptance (\%) for $M_{H}=200 \mathrm{GeV}$

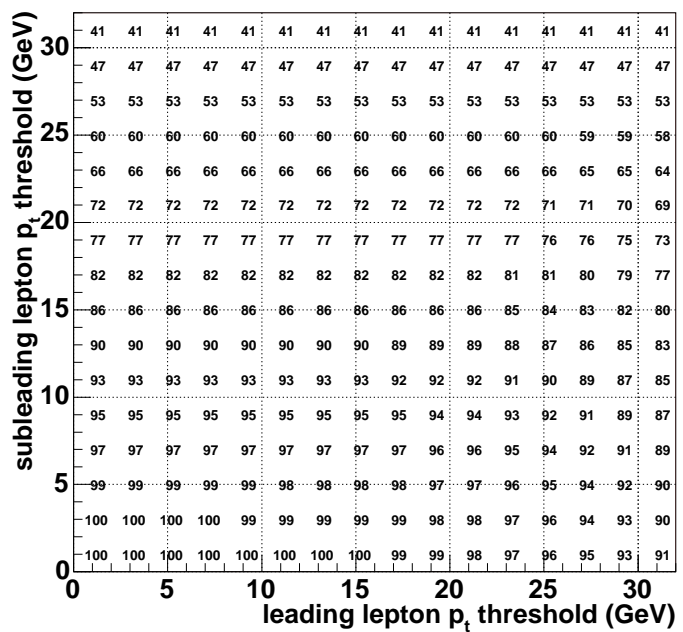

Figure 7.3: Raw $H W W$ signal acceptances as a function of leading and subleading lepton $E_{t}$ thresholds for $m_{H}=110,140,170$ and $200 \mathrm{GeV}$. 

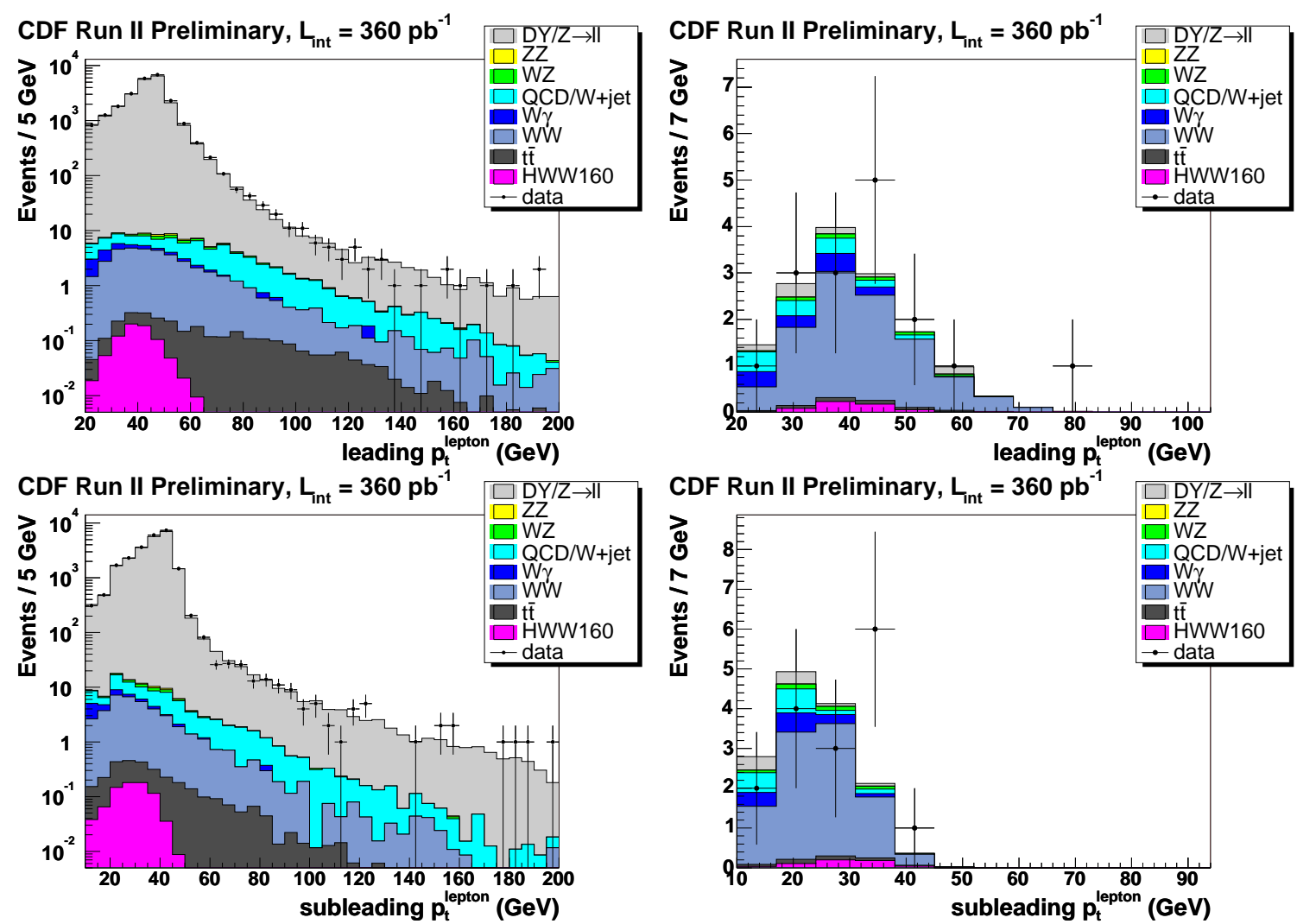

Figure 7.4: Initial (left) and final (right) leading (upper) and subleading (lower) lepton $E_{t}$ distributions for $m_{H}=160 \mathrm{GeV}$.

4. the triggers in use, which have been very well understood and used by the CDF collaboration, required at least one lepton in the event with $E_{t}>20 \mathrm{GeV}$ for a plateau trigger efficiency.

\subsubsection{Charge Signs}

The leptons were required to have charge signs opposite to each other, which reduced the $W Z$ and $Z Z$ diboson background and halved the $W+\gamma /$ jet and QCD background. The $W Z$ and $Z Z$ processes can result in events with same-sign dileptons and large amounts of missing transverse energy if not all of the leptons are identified. 
The $W+\gamma /$ jet and QCD background can look like same-sign dilepton events because photons and jets are not charge-distinguishable.

\subsection{Jets}

As extracted from the $\mathrm{N}-1^{1}$ jet multiplicity distribution shown in Figure 7.5 and listed in Table 7.1, 98.5\% of events that passed the N-1 event selection cuts had less

${ }^{1} \mathrm{~N}-1$ means "obtained by applying to events all the selection cuts but not the one that would use the variable under investigation".

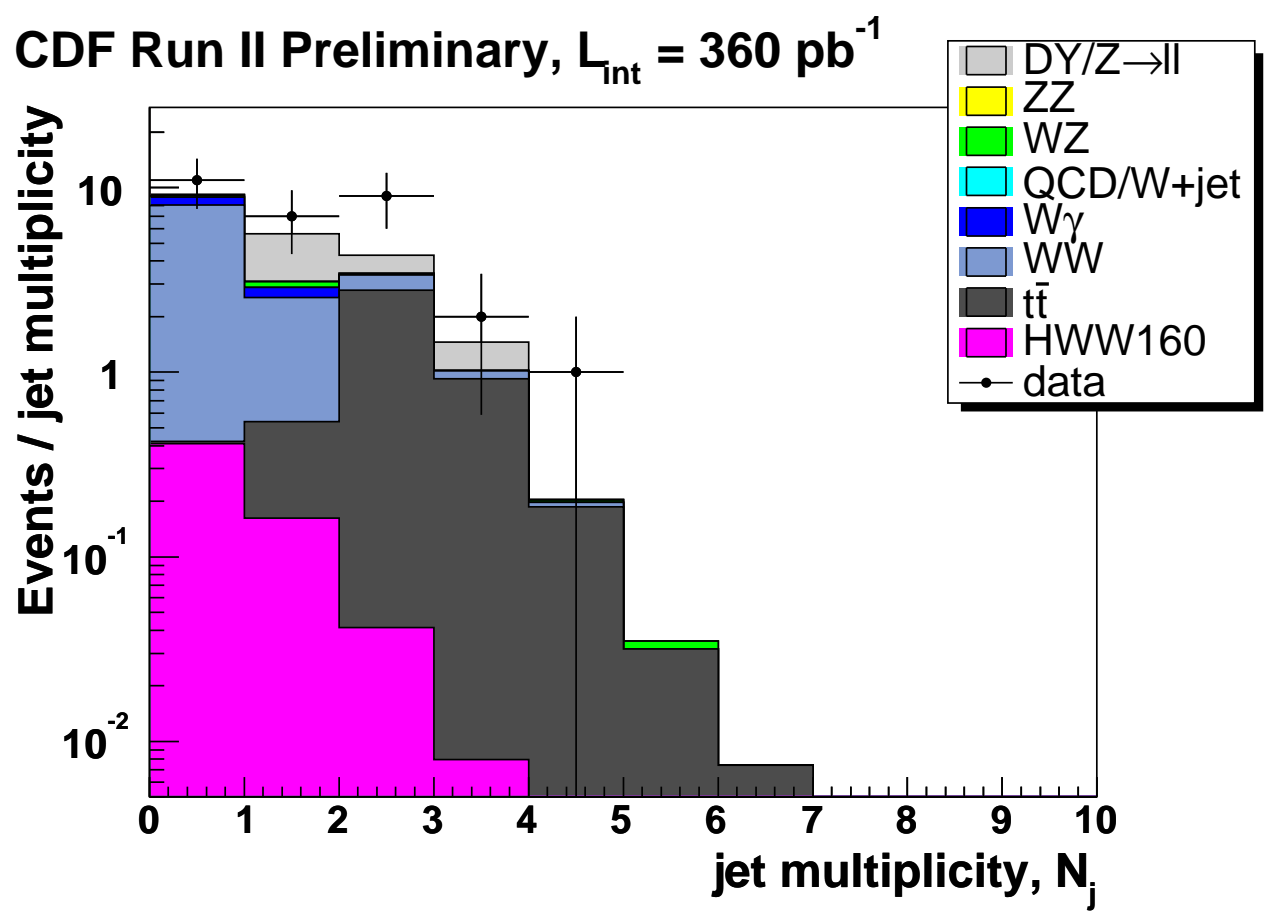

Figure 7.5: N-1 jet multiplicity distributions for $m_{H}=160 \mathrm{GeV}$.

\begin{tabular}{|c||c|c|c|c|}
\hline \hline$m_{H}=160 \mathrm{GeV}$ & $N_{j}=0$ & $N_{j}=1$ & $N_{j}=2$ & $N_{j} \geq 3$ \\
\hline \hline event fraction (\%) & $65.8 \pm 0.2$ & $26.0 \pm 0.2$ & $6.7 \pm 0.1$ & $1.5 \pm 0.1$ \\
\hline \hline
\end{tabular}

Table 7.1: Signal MC event fraction as a function of jet multiplicity. 

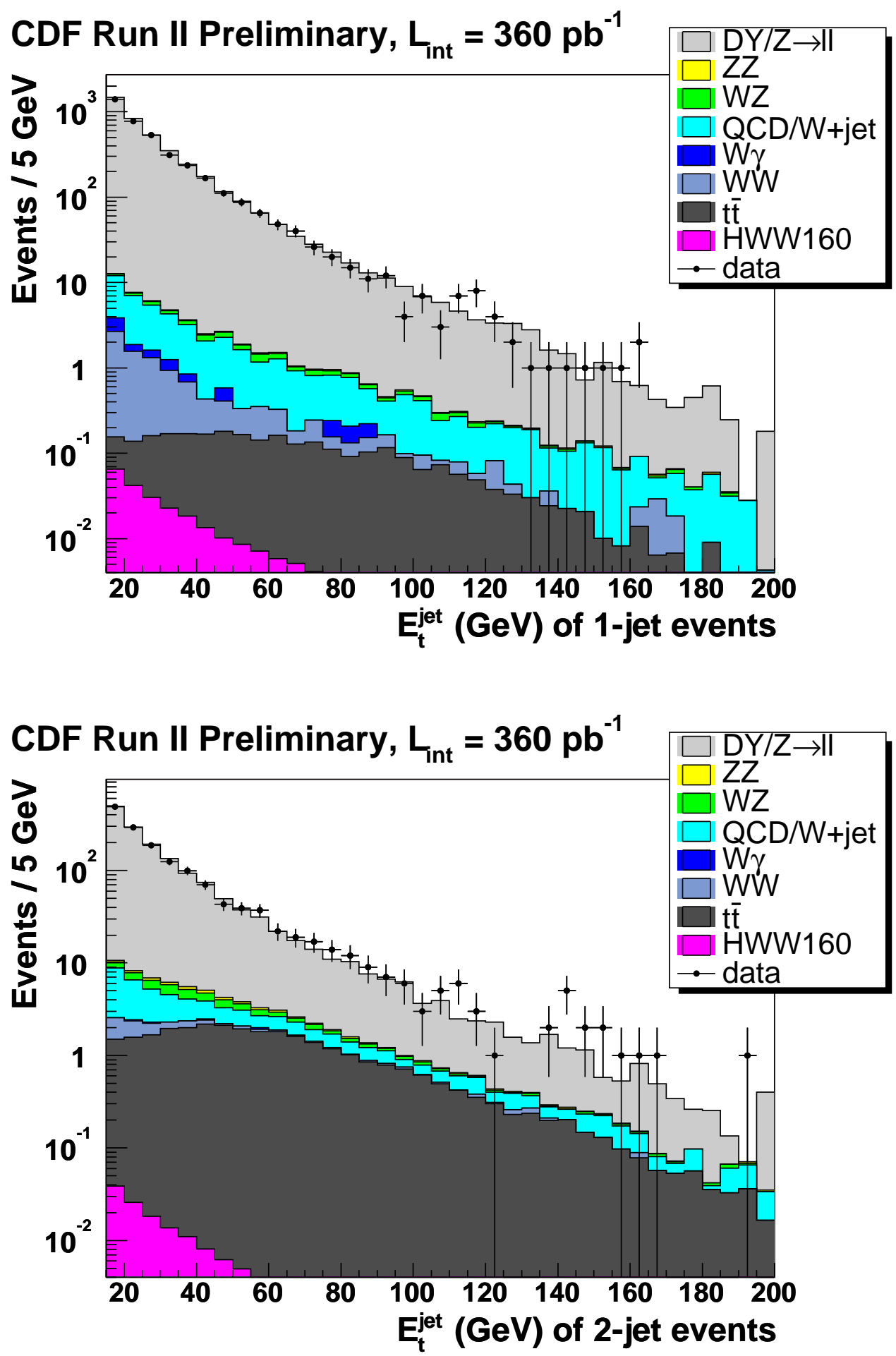

Figure 7.6: Jet $E_{t}$ distributions of 1-jet (upper) and 2-jet (lower) events for $m_{H}=$ $160 \mathrm{GeV}$. 

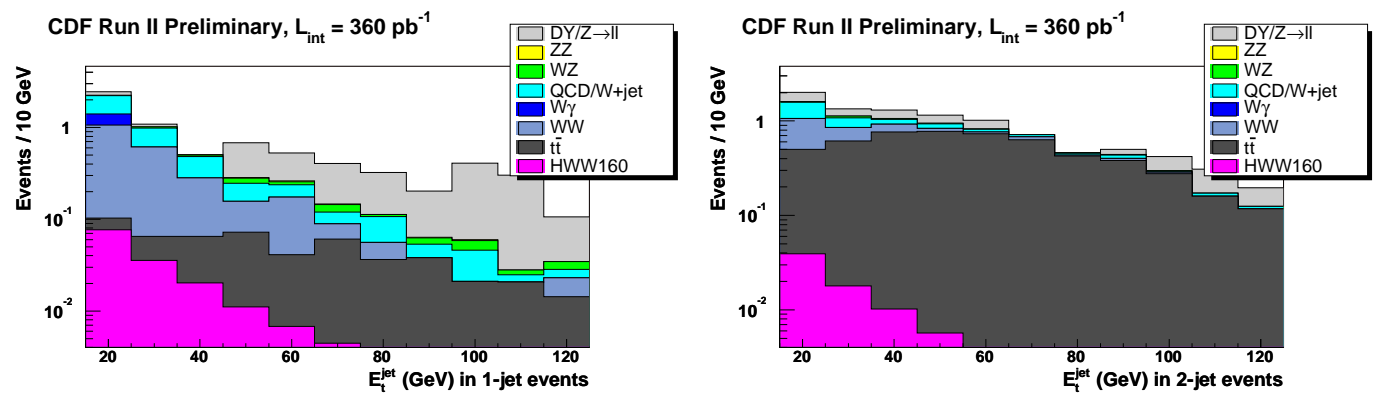

$S / \sqrt{B^{1-j e t ~ e v e n t s ~}}$ vs $E_{t}^{1-j e t}$ Threshold for $M_{H}=110 \mathrm{GeV}$

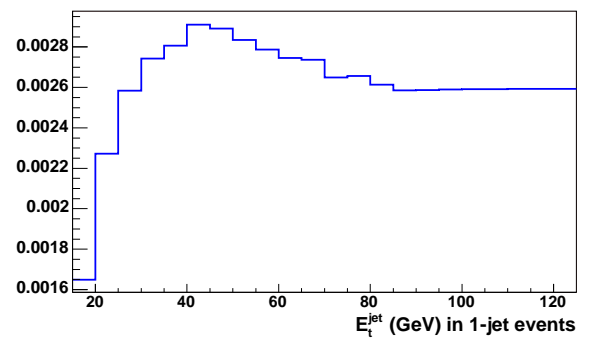

$S / \sqrt{B}^{2-\text { jet events }}$ vs $E_{t}^{2 \text {-jet }}$ Threshold for $M_{H}=110 \mathrm{GeV}$
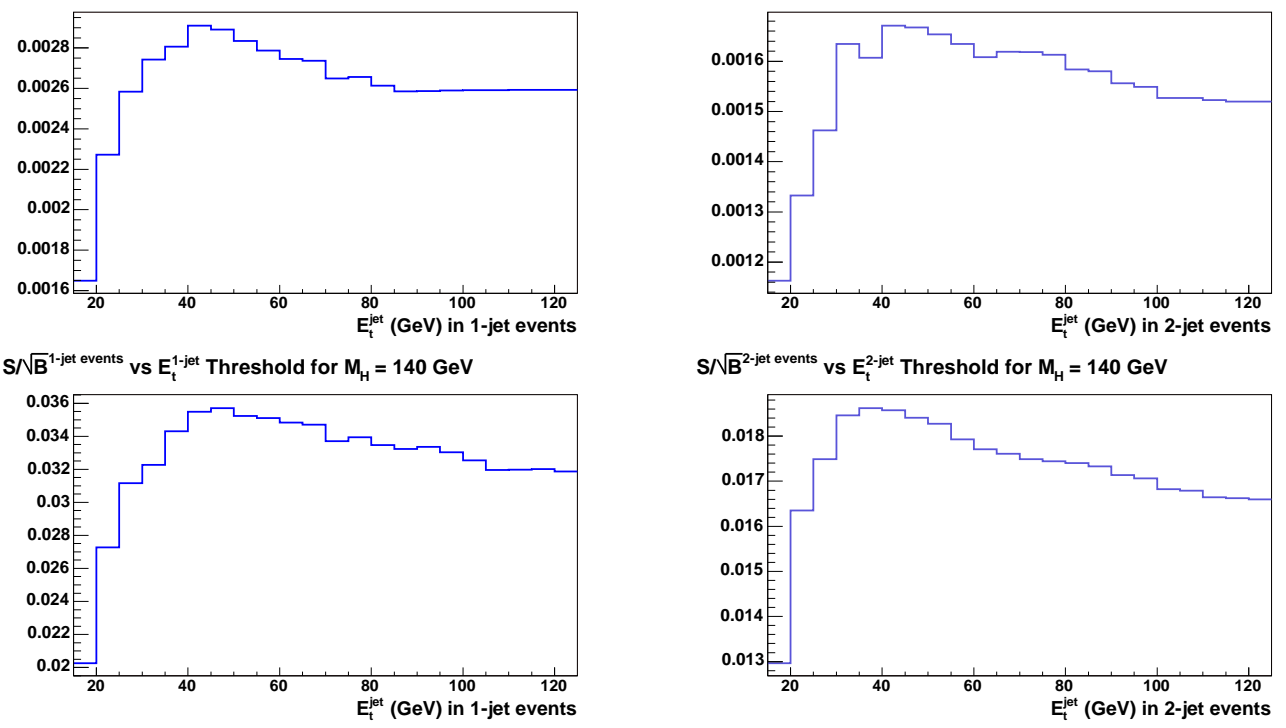

$S / \mathbb{B}^{2 \text {-jet events }}$ vs $E_{t}^{2 \text {-jet }}$ Threshold for $M_{H}=140 \mathrm{GeV}$
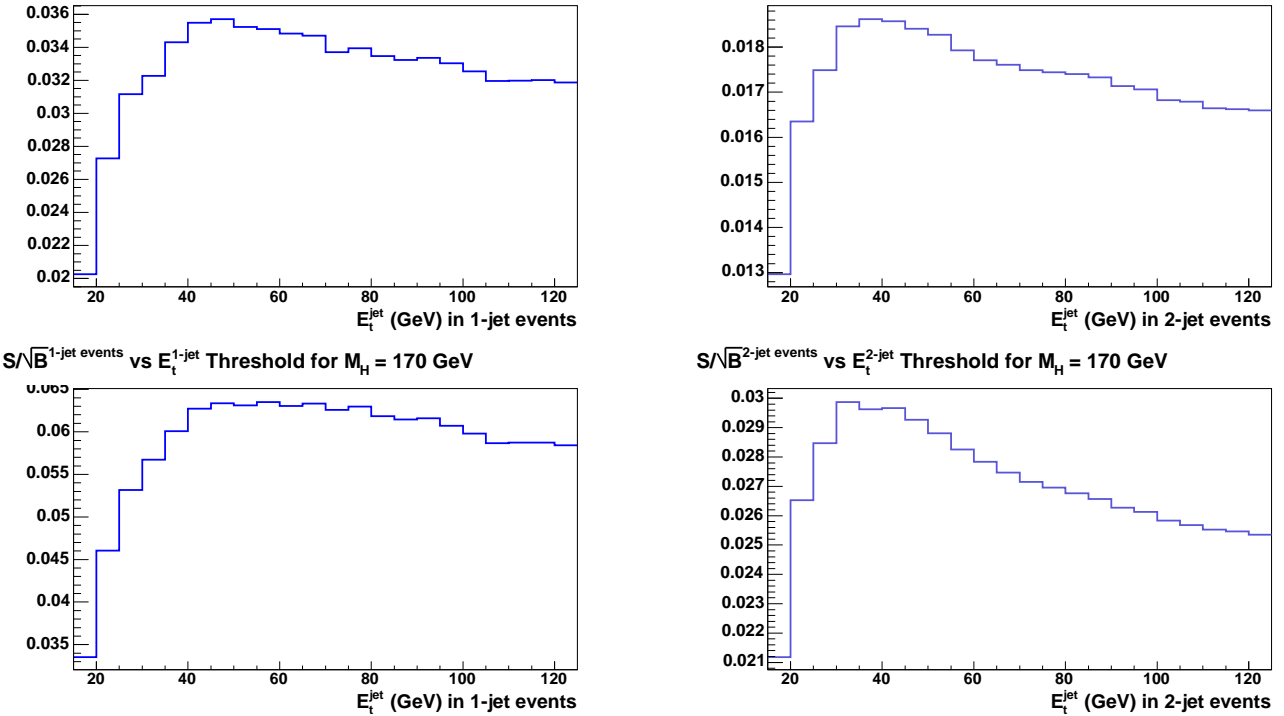

$S / \sqrt{B}^{2 \text {-jet events }}$ vs $E_{t}^{2 \text {-jet }}$ Threshold for $M_{H}=170 \mathrm{GeV}$

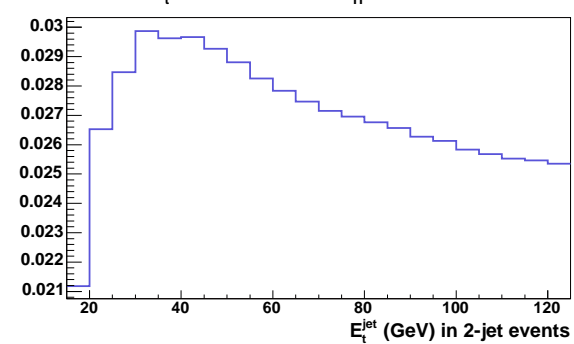

$S / \sqrt{B}^{1-\text { jet events }}$ vs $E_{t}^{1-j e t}$ Threshold for $M_{H}=200 \mathrm{GeV}$
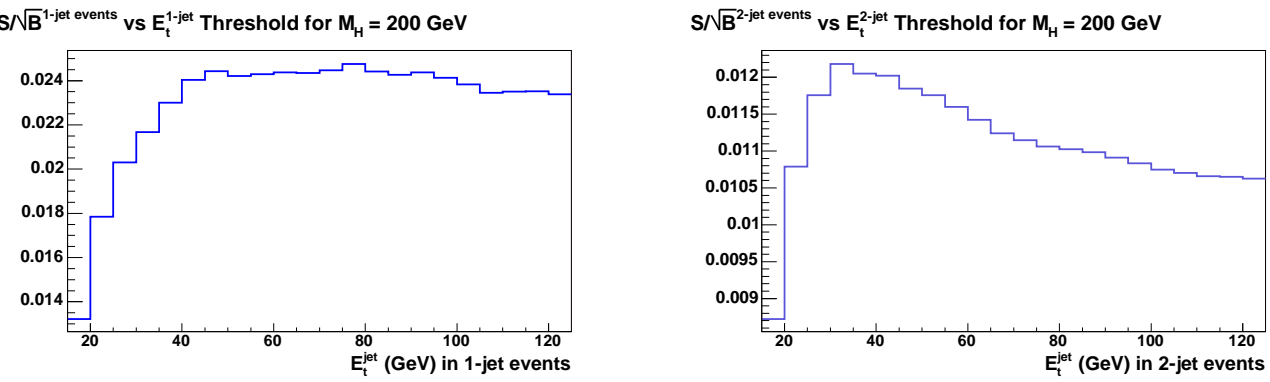

Figure 7.7: N-1 jet $E_{t}$ distributions for $m_{H}=160 \mathrm{GeV}$ and $\mathrm{S} / \sqrt{\mathrm{B}}$ as a function of jet $E_{t}$ in 1-jet (left) and 2-jet (right) bins for $m_{H}=110,140,170$ and $200 \mathrm{GeV}$. 
than 3 jets with $E_{t}>15 \mathrm{GeV}$ and $|\eta|<2.5$; the remaining $1.5 \%$ would only reduce $\mathrm{S} / \sqrt{\mathrm{B}}$ if included. Therefore the analysis covered only the jet multiplicity range of $N_{j} \leq 2$

The $H \rightarrow W^{+} W^{-} \rightarrow \ell^{+} \nu \ell^{-} \bar{\nu}$ signal has only soft jets in the final state due to initial state radiation. The major jet background was Drell-Yan $Z / \gamma^{*} \rightarrow \ell^{+} \ell^{-}$with jets from initial or final state radiation; the major cause of different jet $E_{t}$ spectrum shapes in 1-jet and 2-jet bins was $t \bar{t} \rightarrow W^{+} W^{-} b \bar{b} \rightarrow \ell^{+} \nu \ell^{-} \bar{\nu} b \bar{b}$ with two hard $b$-jets in the final state, as shown in Figure 7.6.

The cut on jet $E_{t}$ was made per jet multiplicity bin for the best $\mathrm{S} / \sqrt{\mathrm{B}}$ :

$$
\begin{aligned}
& N_{j}=0 ; \\
& N_{j}=1 \text { and } E_{t}^{j}<55 \mathrm{GeV} ; \\
& N_{j}=2 \text { and } E_{t}^{j}<40 \mathrm{GeV} .
\end{aligned}
$$

$\mathrm{S} / \sqrt{\mathrm{B}}$ as a function of jet $E_{t}$ per jet multiplicity bin are shown in Figure 7.7.

\subsection{Dilepton Invariant Mass}

The requirement of dilepton invariant mass $m_{\ell \ell}>16 \mathrm{GeV}$ was made to keep the analysis away from the background related to $b$ quarks, the major components of which were $\Upsilon$ and $b \bar{b}$ events.

The requirement of dilepton invariant mass to be approximately smaller than half of the Higgs mass was made for the following reason: as explained in Section 7.1, due to the spin constraint of the Higgs boson, the two charged leptons from the signal tend to fly parallel to each other and so do the two neutrinos; the lepton pair and 
the neutrino pair, both of which are massless in comparison to the Higgs boson, tend to fly back-to-back, each carrying away energy $E \sim \frac{1}{2} m_{H}$ in the Higgs boson's rest frame. The $H W W$ dilepton invariant mass, defined as $m_{\ell \ell}=\sqrt{E_{\ell \ell}{ }^{2}-p_{\ell \ell}{ }^{2}}$, has a relation to the Higgs mass as $m_{\ell \ell} \lesssim \frac{1}{2} m_{H}$. Since no such spin constraint exhibits in the background processes, the dilepton invariant mass from the background has no tendency of being small or large. The $H W W$ signal therefore can be discriminated from the background using $m_{\ell \ell} \lesssim \frac{1}{2} m_{H}$. As shown in Figure 7.8 and Figure 7.9 , the $H W W$ dilepton invariant mass mostly distributes up to half of the Higgs mass.

$\mathrm{S} / \sqrt{\mathrm{B}}$ was scanned over the $m_{\ell \ell}$ upper cut location for the maximal $\mathrm{S} / \sqrt{\mathrm{B}}$ improvement at each of the investigated Higgs masses. Since the dilepton invariant mass from the signal was directly correlated with the Higgs mass, the $\mathrm{S} / \sqrt{\mathrm{B}}-\mathrm{maximal} m_{\ell \ell}$

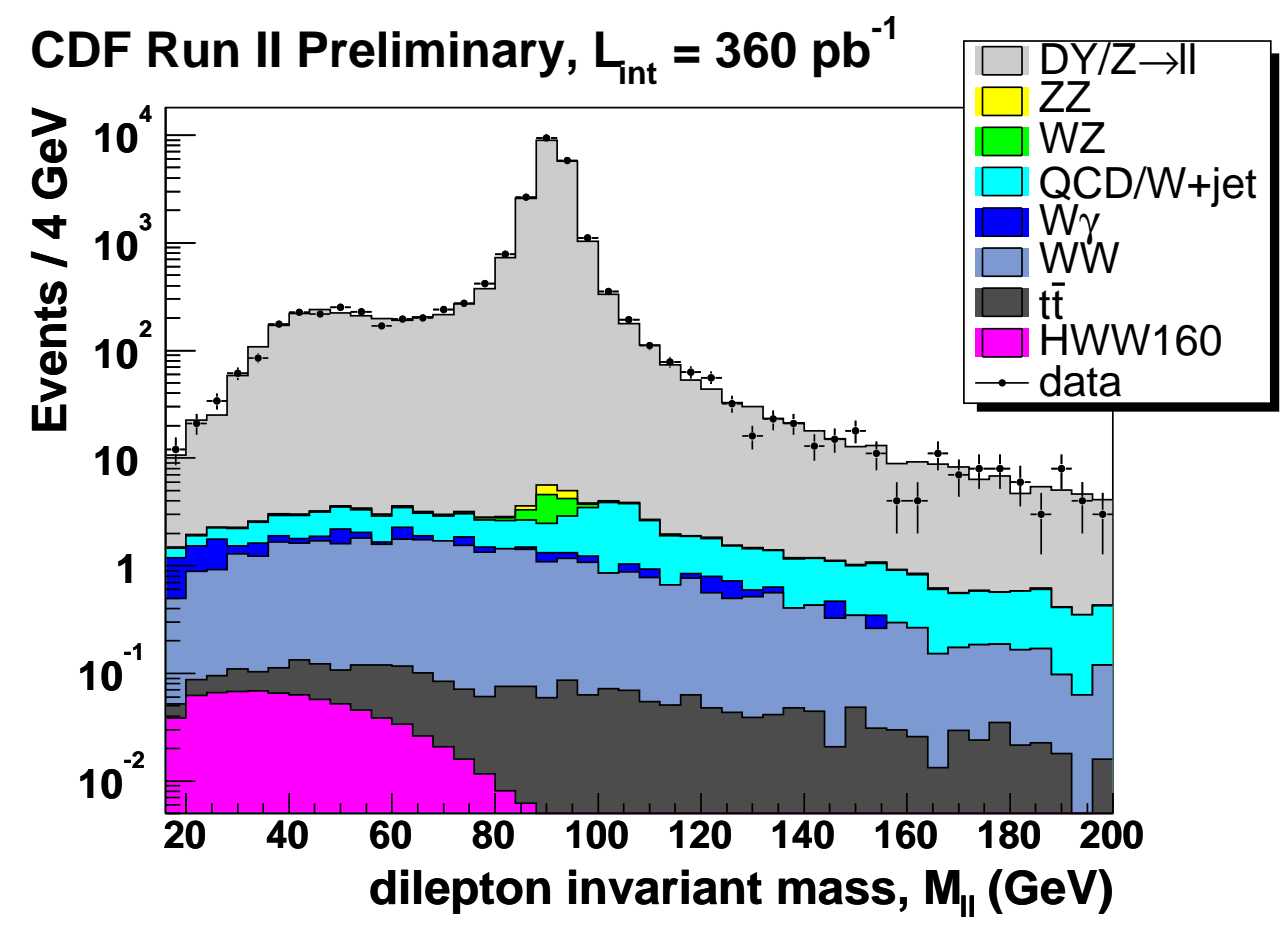

Figure 7.8: Initial dilepton invariant mass $m_{\ell \ell}$ distributions for $m_{H}=160 \mathrm{GeV}$. 


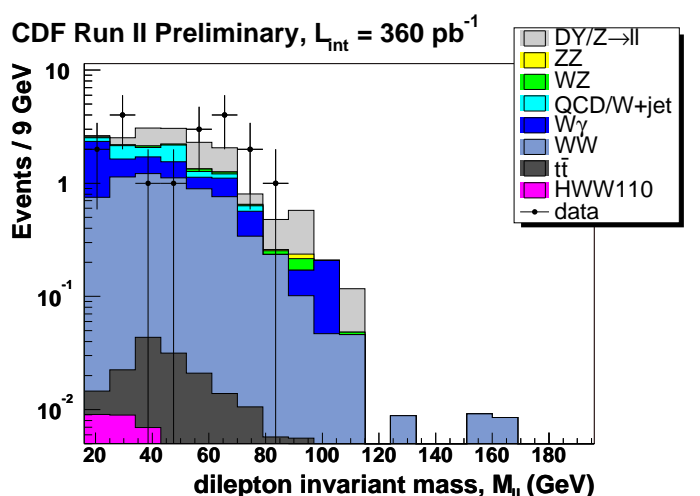

$S / \sqrt{B}\left(M_{I l}\right.$ upper cut $)$ for $M_{H}=110 \mathrm{GeV}$

CDF Run II Preliminary, $L_{\text {int }}=360$ pb $^{-1}$
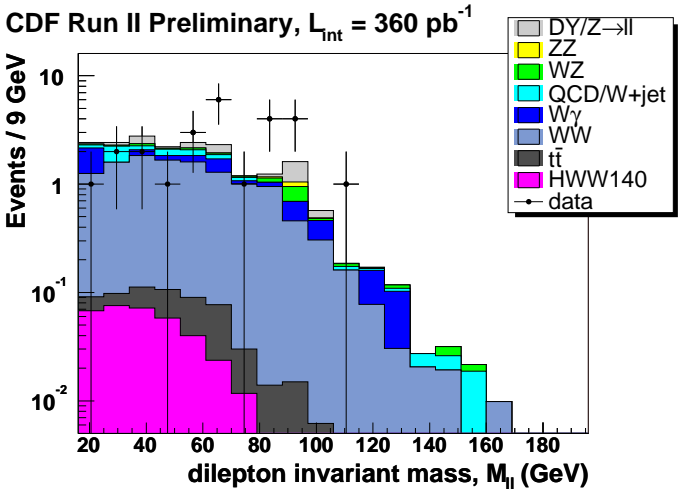

CDF Run II Preliminary, $L_{\text {nt }}=360$ pb $^{-1}$
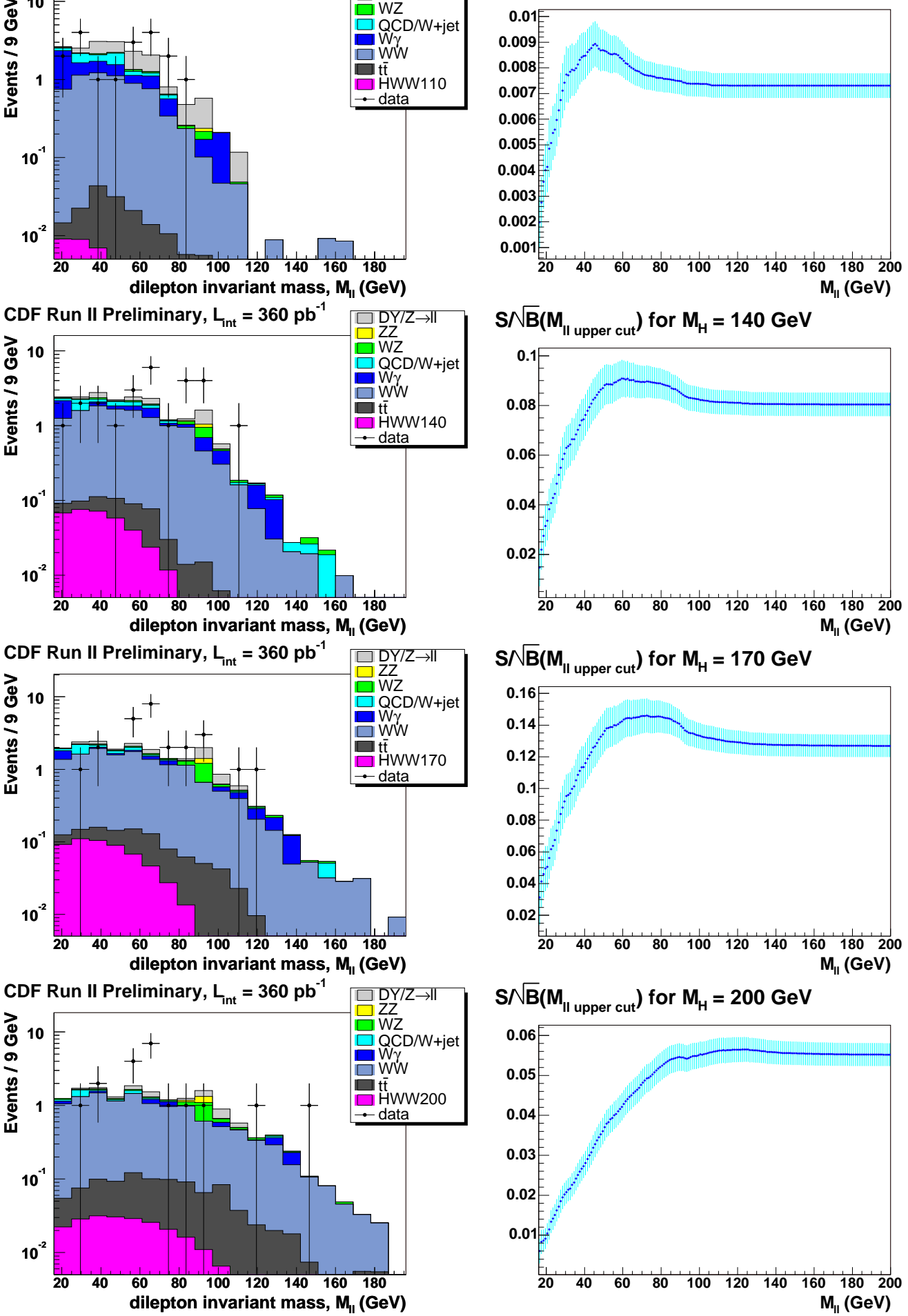

$S / \sqrt{B}\left(M_{11}\right.$ upper cut $)$ for $M_{H}=140 \mathrm{GeV}$

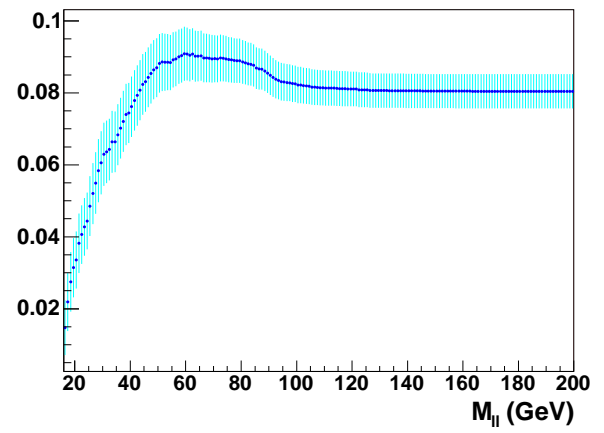

$S / \sqrt{B}\left(M_{11}\right.$ upper cut $)$ for $M_{H}=170 \mathrm{GeV}$

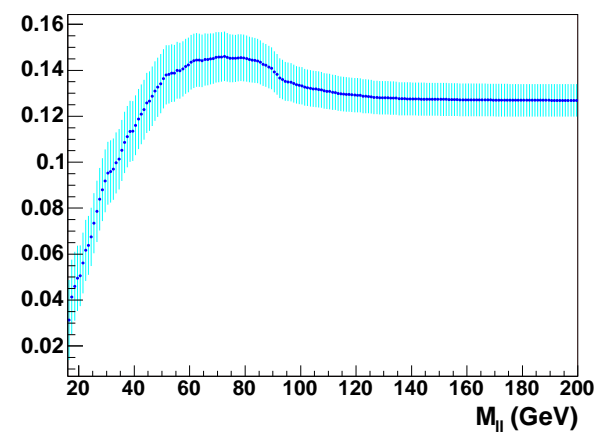

$S / \sqrt{B}\left(M_{\text {Il upper cut }}\right)$ for $M_{H}=200 \mathrm{GeV}$

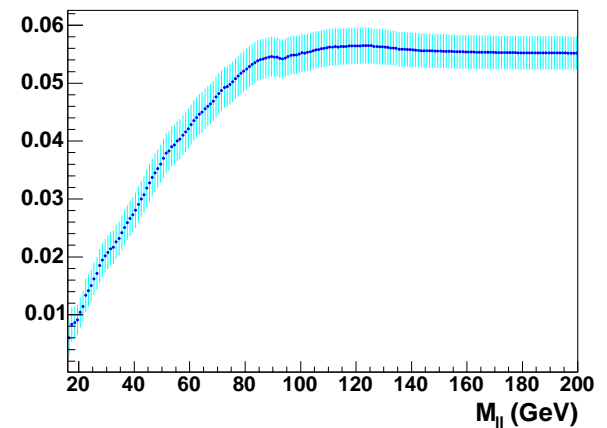

Figure 7.9: N-1 $m_{\ell \ell}$ distributions and $\mathrm{S} / \sqrt{\mathrm{B}}$ as a function of $m_{\ell \ell}$ upper cut location for $m_{H}=110,140,170$ and $200 \mathrm{GeV}$. 


\section{$S / \sqrt{B}\left(M_{\| l}\right.$ cut $)$ vs $M_{H}$}

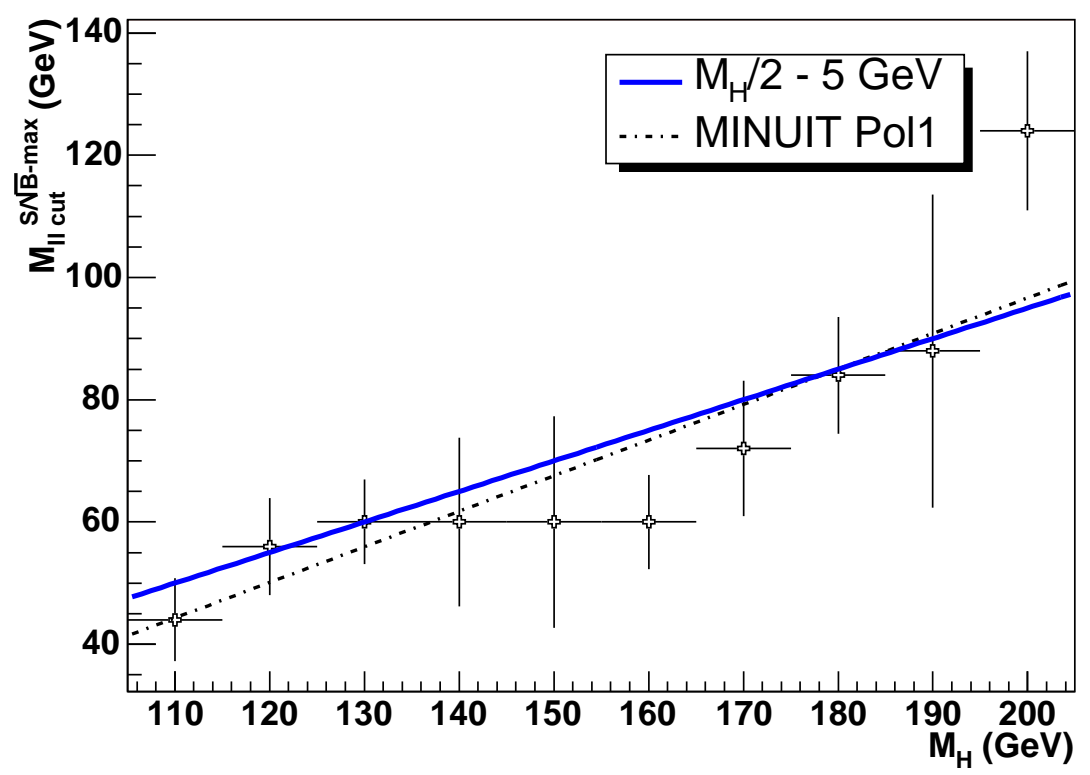

Figure 7.10: $\mathrm{S} / \sqrt{\mathrm{B}}$-maximal $m_{\ell \ell}$ upper cut location along with systematic uncertainties as a function of the Higgs mass. The requirement of $m_{\ell \ell}<\frac{1}{2} m_{H}-5 \mathrm{GeV}$ appears optimal for most of the analyzed Higgs mass points.

upper cut location moved along the Higgs mass. Therefore the $m_{\ell \ell}$ upper cut was made $m_{H}$-dependent and was simplified from the $\mathrm{S} / \sqrt{\mathrm{B}}$-maximal $m_{\ell \ell}$ upper cut locations, as shown in Figure 7.9 and Figure 7.10. The requirement of dilepton invariant mass $m_{\ell \ell}<\frac{1}{2} m_{H}-5 \mathrm{GeV}$ was made to keep the analysis within the $H W W$ dilepton mass region and, therefore, to retain good $\mathrm{S} / \sqrt{\mathrm{B}}$ and the best achievable expected limits. 


\subsection{Missing Transverse Energy}

The $H \rightarrow W^{+} W^{-} \rightarrow \ell^{+} \nu \ell^{-} \bar{\nu}$ signal has two neutrinos in the final state that cause a large amount of real ${ }^{2}$ missing transverse energy. Moreover, the two neutrinos from the signal tend to fly parallel to each other so that the missing transverse energy is larger than without the spin constraint, in contrast to the dominant inclusive SM $W W$ dilepton background. Missing transverse energy distributions at the initial level are shown in Figure 7.11 and Figure 7.12.

The greater the Higgs mass, the larger the energy carried away by the neutrinos from the signal and therefore the larger the missing transverse energy in the final state. So the most optimal $\not_{t}$ cut was expected to be $m_{H}$-dependent.

\footnotetext{
${ }^{2}$ Real $\not_{t}$ is in contrast to instrumental $\not_{t}$, as explained in Section 5.3.
}

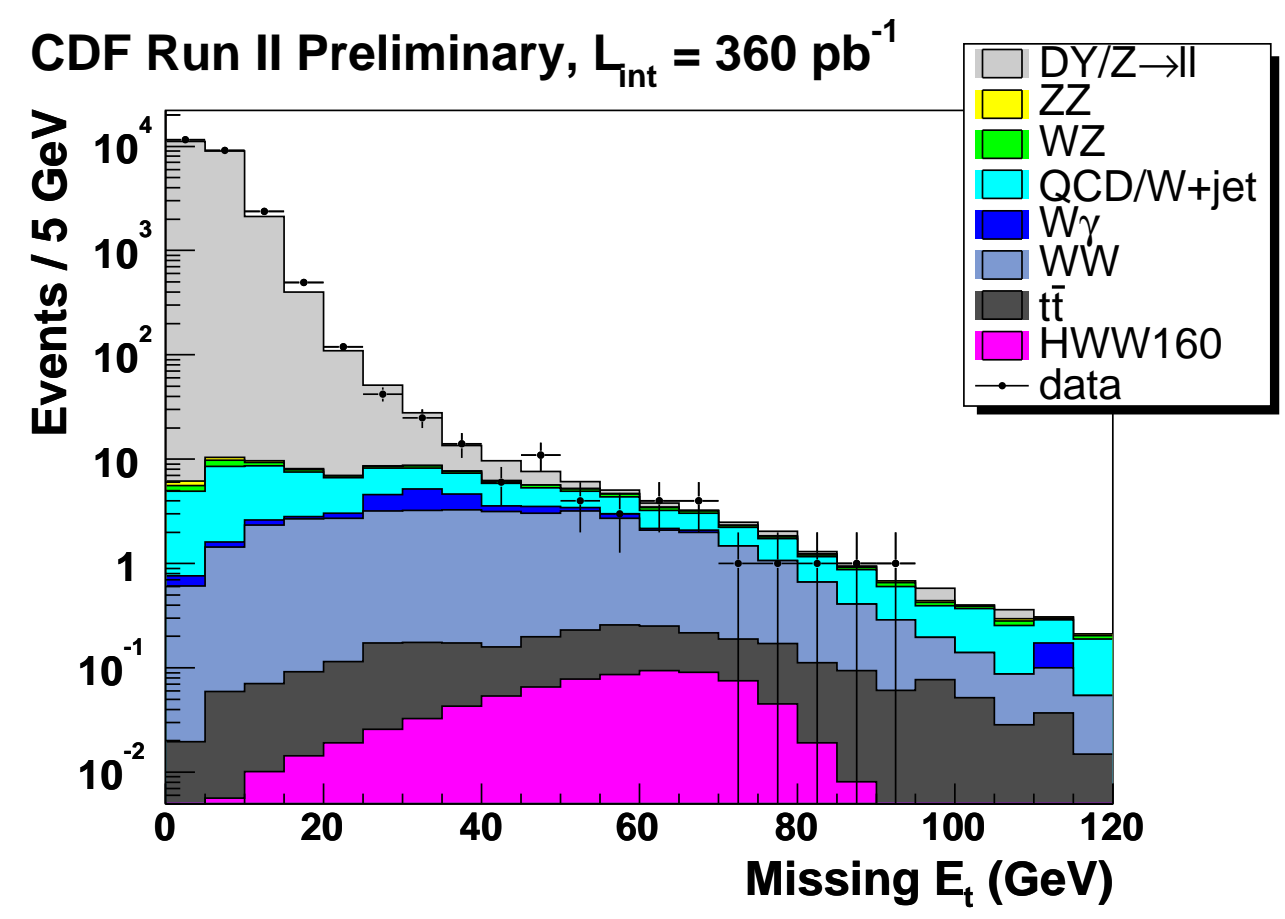

Figure 7.11: Initial missing transverse energy $\not_{t}$ distributions for $m_{H}=160 \mathrm{GeV}$. 


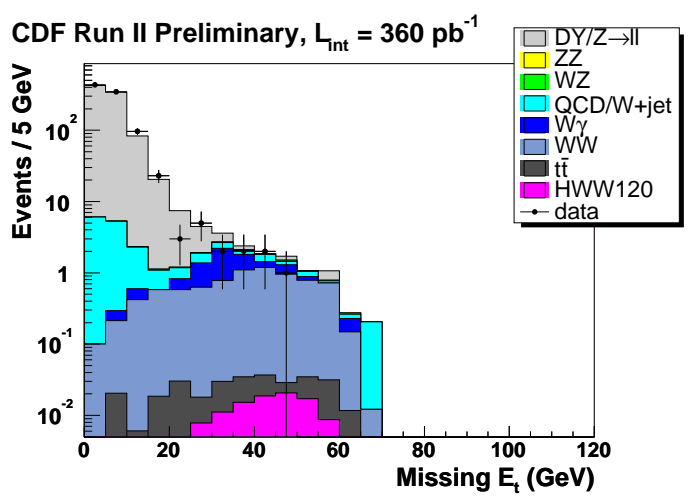

$S / \sqrt{B}$ (Missing $E_{t}$ Cut) for $M_{H}=120 \mathrm{GeV}$

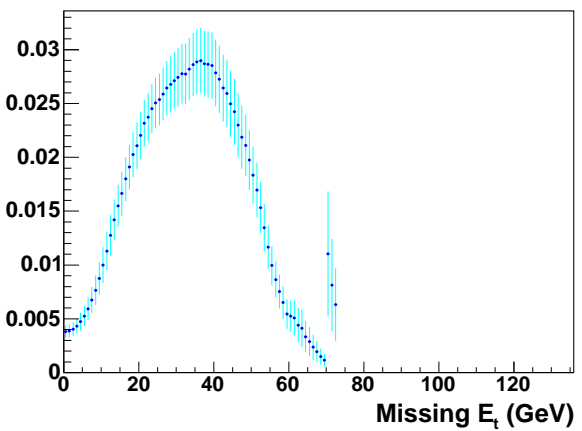

CDF Run II Preliminary, $L_{\text {int }}=360 \mathrm{pb}^{-1}$

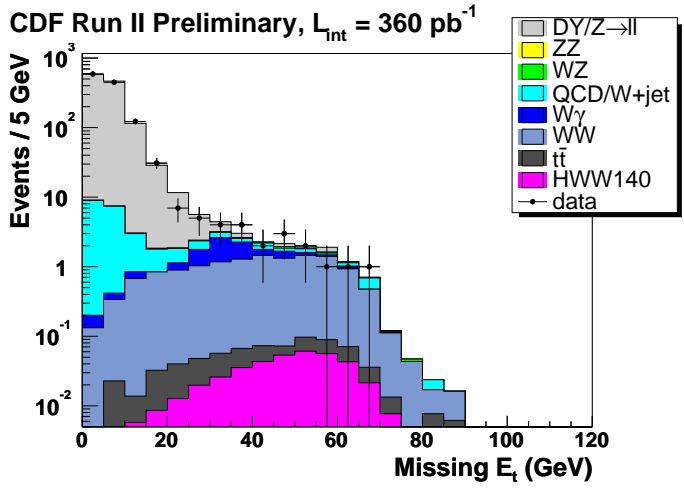

$S / \sqrt{B}$ (Missing $E_{1}$ Cut) for $M_{H}=140 \mathrm{GeV}$
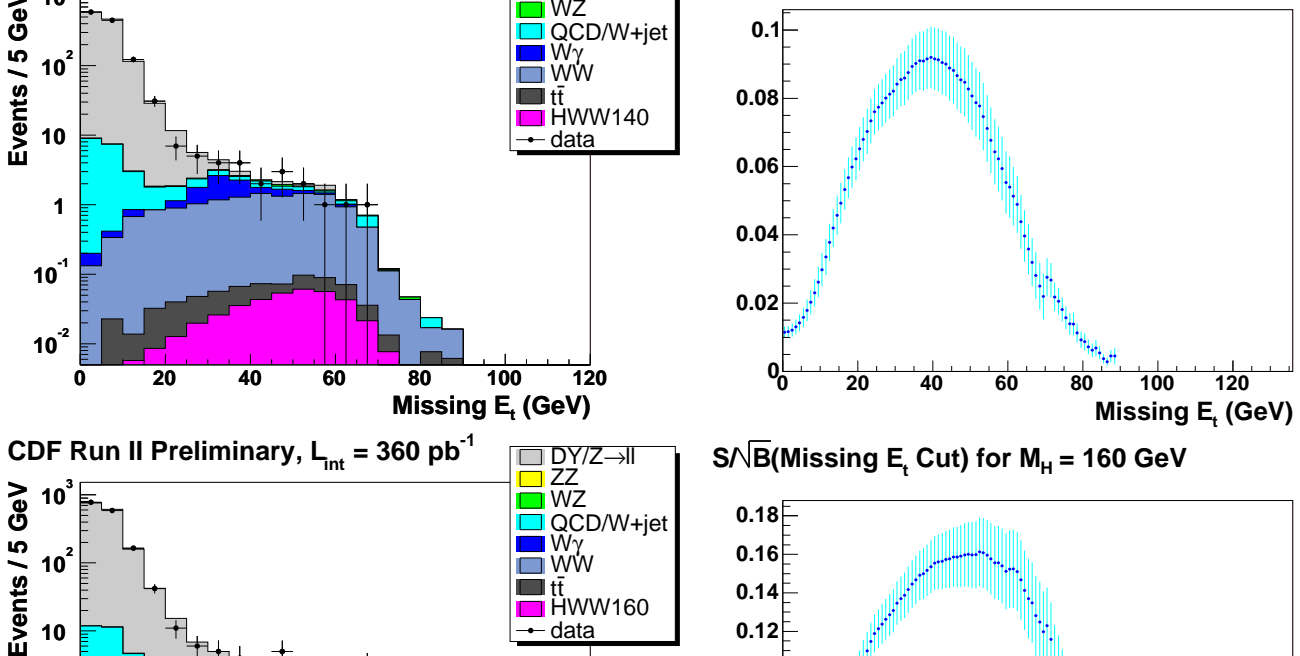

$S / \sqrt{B}$ (Missing $E_{t}$ Cut) for $M_{H}=160 \mathrm{GeV}$
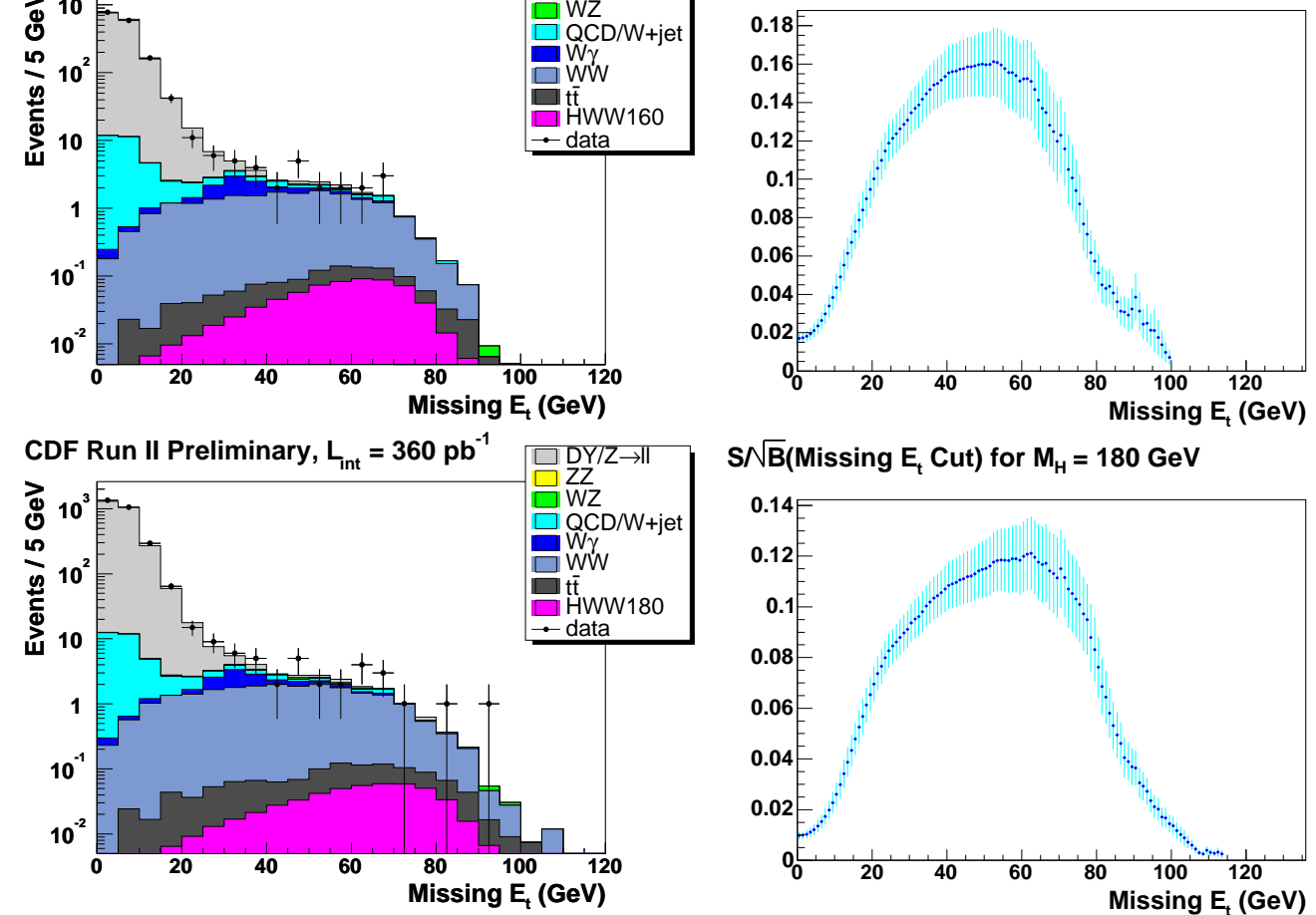

$S / \sqrt{B}$ (Missing $E_{t}$ Cut) for $M_{H}=180 \mathrm{GeV}$

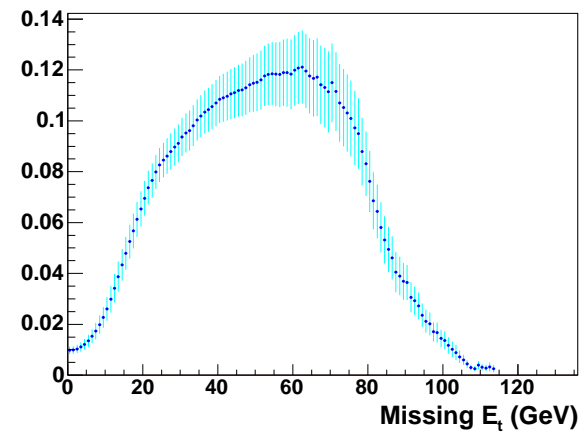

Figure 7.12: N-1 $E_{t}$ distributions and $\mathrm{S} / \sqrt{\mathrm{B}}$ as a function of $E_{t}$ cut location for $m_{H}=120,140,160$ and $180 \mathrm{GeV}$. 


\begin{tabular}{|c|c|c|c|c|c|c|c|c|c|}
\hline$m_{H}$ & \multicolumn{9}{|c|}{ the constant in the cut formula of $\not E_{t}>\frac{1}{4} m_{H}+$ constant $(\mathrm{GeV})$} \\
\hline$(\mathrm{GeV})$ & -10 & -5 & $\mathbf{0}$ & +5 & +10 & +15 & +20 & +25 & +30 \\
\hline 120 & 8.59 & 8.72 & 8.40 & 8.02 & 8.28 & 9.75 & 13.55 & 26.68 & 42.58 \\
\hline 140 & 6.36 & 6.11 & 5.70 & 5.77 & 6.11 & 6.64 & 8.22 & 12.38 & 25.35 \\
\hline 160 & 4.28 & 4.14 & 3.95 & 3.90 & 3.95 & 4.16 & 4.51 & 5.53 & 7.84 \\
\hline 180 & 4.26 & 4.00 & 3.99 & 3.90 & 3.84 & 3.91 & 4.29 & 5.30 & 6.95 \\
\hline
\end{tabular}

Table 7.2: Pseudoexperimental upper limits on the $H W W$ production cross-section as a function of the contant in the cut formula of $\mathbb{E}_{t}>\frac{1}{4} m_{H}+$ constant for $m_{H}=$ $120,140,160$ and $180 \mathrm{GeV}$.

The procedure of finding the best $\not_{t}$ cut was as follows:

1. the $\not_{t}$ cut location that would yield the best results for each mass was estimated by observation and formulated to be simply $\not_{t} \gtrsim \frac{1}{4} m_{H}+$ constant;

2. the expected limit as well as $\mathrm{S} / \sqrt{\mathrm{B}}$ was scanned over the constant per $5 \mathrm{GeV}$, as shown in Table 7.2 , and $\mathscr{E}_{t}>\frac{1}{4} m_{H}$ was found to yield good signal acceptance, $\operatorname{good} \mathrm{S} / \sqrt{\mathrm{B}}$ and almost always the best expected limits.

\subsection{Azimuthal Angle between $\not_{t}$ and Other Objects}

The cut of $E_{t}>50 \mathrm{GeV}$ or $\Delta \phi_{E_{t}, \ell / j}>20^{\circ}$ was employed to suppress the Drell-Yan dilepton background for the following reasons:

$Z / \gamma^{*} \rightarrow e^{+} e^{-}$: final state radiation plus photon/jet energy mis-measurement results in a small amount of missing transverse energy going close to the radiation source electron;

$Z / \gamma^{*} \rightarrow \tau^{+} \tau^{-} \rightarrow \ell^{+} \nu_{\ell} \bar{\nu}_{\tau} \ell^{-} \bar{\nu}_{\ell} \nu_{\tau}$ where $\ell \in\{e, \mu\}$ : real missing transverse energy in a small amount also tends to go close to either lepton. 

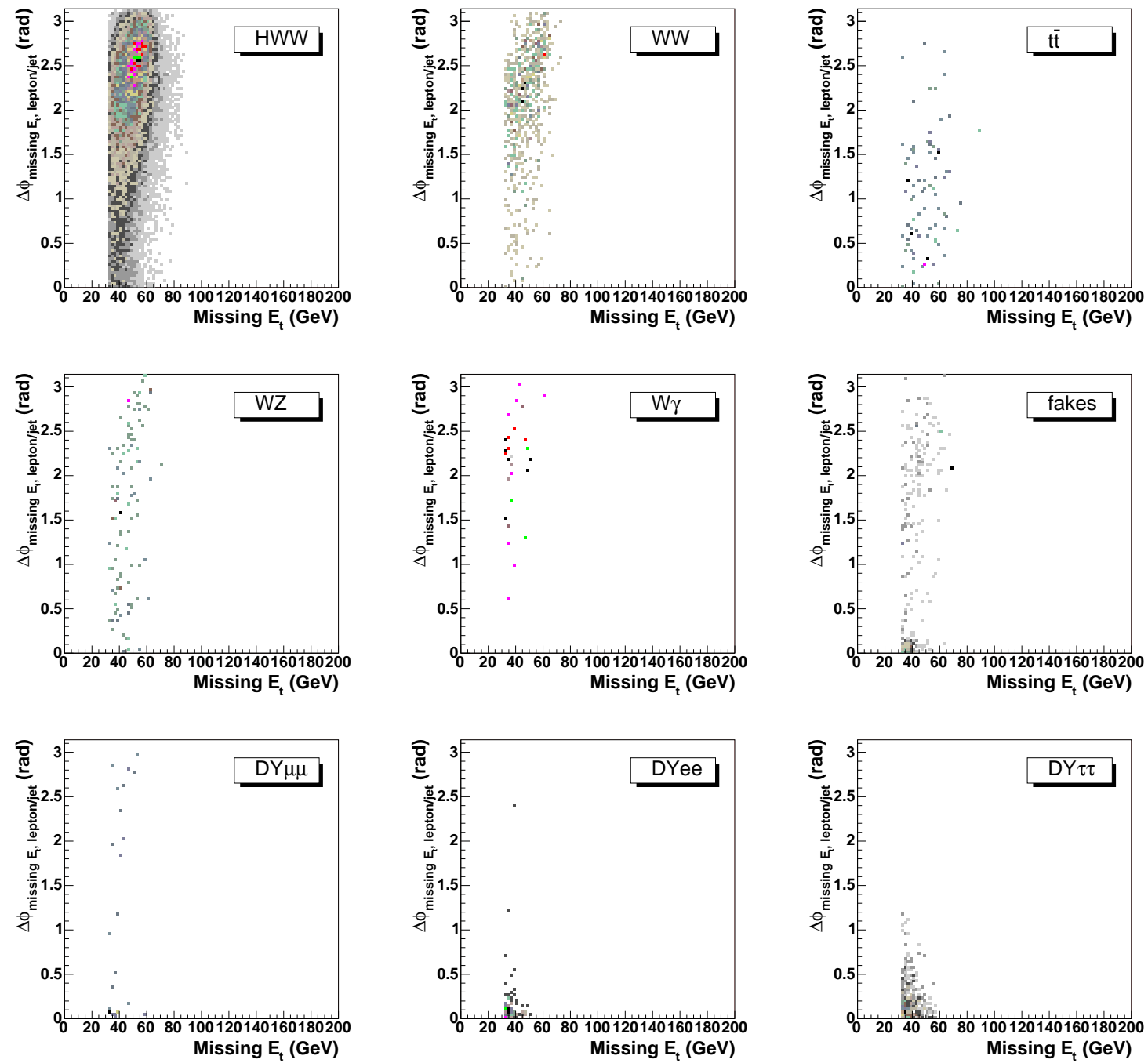

Figure 7.13: N-1 $\not_{t}$ vs. $\Delta \phi_{E_{t}, \ell / j}$ distributions of each signal or background process for $m_{H}=130 \mathrm{GeV}$. 


\begin{tabular}{|l|r|r|r|r|r|r|r|r|r|r|}
\hline \hline$m_{H}(\mathrm{GeV})$ & 110 & 120 & 130 & 140 & 150 & 160 & 170 & 180 & 190 & 200 \\
\hline \hline DY b. $\Delta \phi$ & 15.25 & 16.08 & 10.32 & 6.96 & 5.33 & 3.34 & 2.23 & 1.80 & 1.20 & 0.96 \\
\hline DY $a . \Delta \phi$ & 2.11 & 1.64 & 1.27 & 1.01 & 1.20 & 0.76 & 0.83 & 0.83 & 0.84 & 0.96 \\
\hline \hline
\end{tabular}

Table 7.3: N-1 event yields of the Drell-Yan dilepton background per analyzed Higgs mass before $(b . \Delta \phi)$ or after $(a . \Delta \phi)$ the $\not_{t}>50 \mathrm{GeV}$ or $\Delta \phi_{\not / t}, \ell / j>20^{\circ}$ cut.

As shown in Figure 7.13, the Drell-Yan $Z / \gamma^{*} \rightarrow\left\{e^{+} e^{-}, \tau^{+} \tau^{-}\right\}$background appears mostly around the corner of soft $\not_{t}$ and small $\Delta \phi_{\#_{t}, \ell / j}$ in contrast to the signal and other background processes.

Reductions of the Drell-Yan dilepton background due to the use of $\not_{t}>50 \mathrm{GeV}$ or $\Delta \phi_{E_{t}, \ell / j}>20^{\circ}$ cut are listed in Table 7.3, appearing $m_{H^{-}}$dependent because of the previous $\mathbb{E}_{t}>\frac{1}{4} m_{H}$. As a result of the large Drell-Yan production cross-section, the reductions at lower masses were tremendous in comparison to the total expected event yields.

\subsection{Sum of Lepton and Missing Transverse Energies}

A loose upper bound on missing transverse energy was placed indirectly for the same reason as stated in Section 7.4, since the only other upper bound, $m_{\ell \ell}<$ $\frac{1}{2} m_{H}-5 \mathrm{GeV}$, did not use any information from the neutrinos.

The $E_{t}^{\ell_{1}}+E_{t}^{\ell_{2}}+E_{t}$ distributions at the initial or N-1 level are shown in Figure 7.14 and Figure 7.15 , where a tendency of signal $E_{t}^{\ell_{1}}+E_{t}^{\ell_{2}}+E_{t}$ almost entirely staying within the Higgs mass is observable. The sum of lepton transverse energy and missing transverse energy was required to be within the Higgs mass, i. e. $E_{t}^{\ell_{1}}+E_{t}^{\ell_{2}}+\not E_{t}<m_{H}$, which improved $\mathrm{S} / \sqrt{\mathrm{B}}$ by up to $10 \%$. 


\subsection{Summary of Event Selection Cuts}

The event selection cuts were applied in the following order:

1. two well-reconstructed leptons with

the trigger one $E_{t}>20 \mathrm{GeV}$;

non-trigger one $E_{t}>10 \mathrm{GeV}$;

2. $m_{\ell \ell}>16 \mathrm{GeV}$;

3. on jets with $E_{t}>15 \mathrm{GeV}$ and $|\eta|<2.5$ :

$N_{j}=0$;

$N_{j}=1$ and $E_{t}^{j}<55 \mathrm{GeV}$

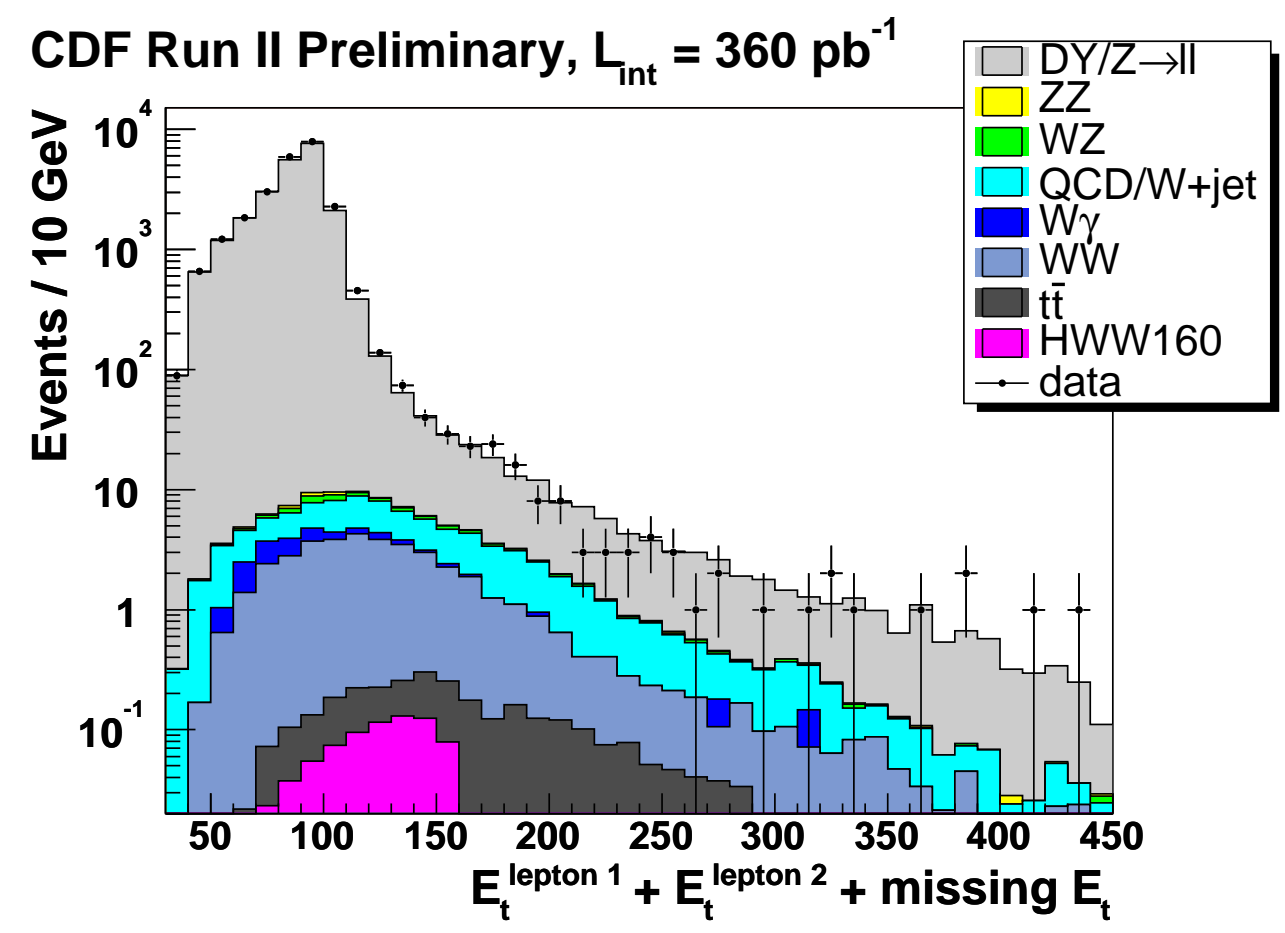

Figure 7.14: Initial $E_{t}^{\ell_{1}}+E_{t}^{\ell_{2}}+E_{t}$ distributions for $m_{H}=160 \mathrm{GeV}$. 

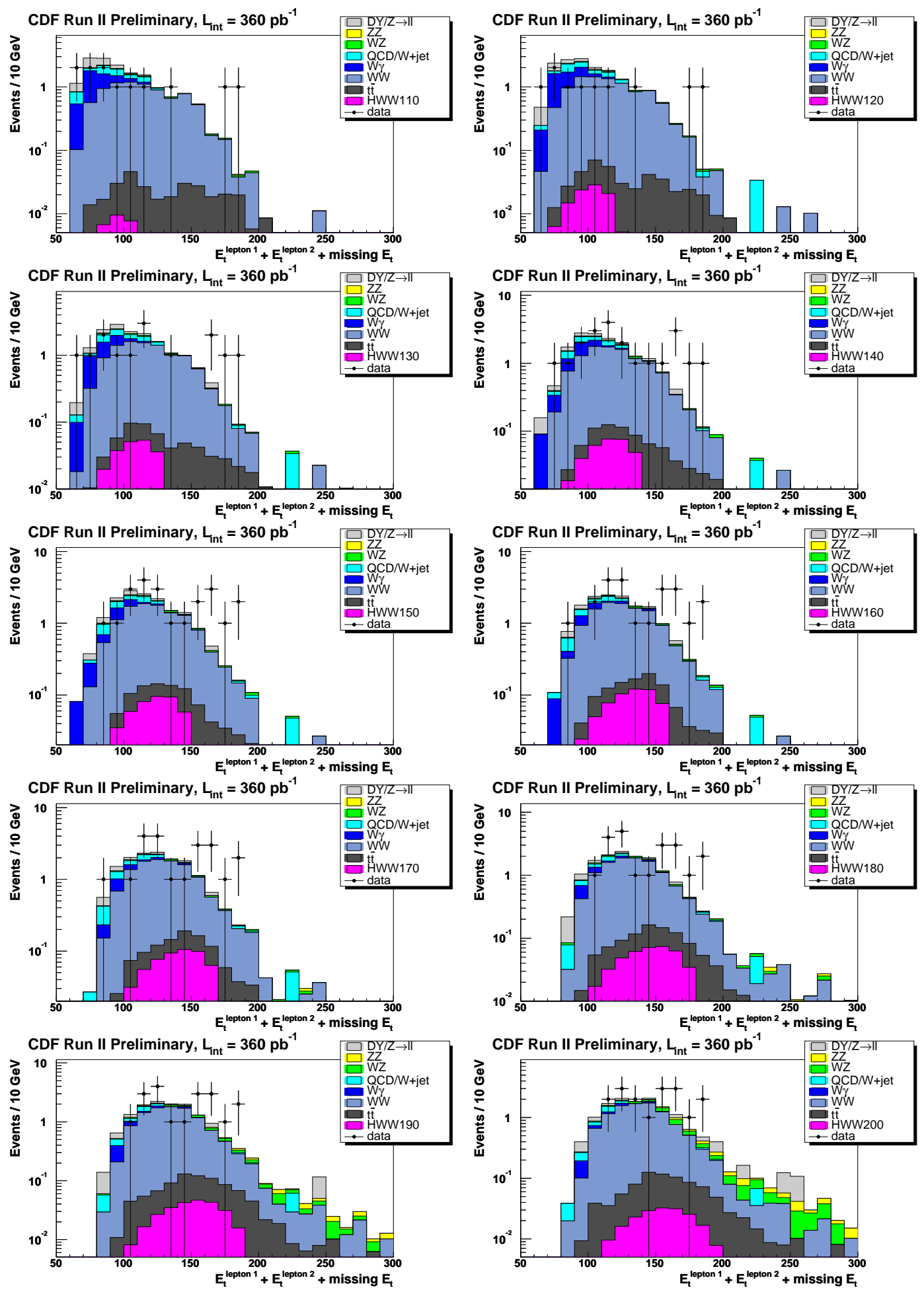

Figure 7.15: N-1 $E_{t}^{\ell_{1}}+E_{t}^{\ell_{2}}+E_{t}$ distributions for each of the analyzed Higgs masses. 


$$
N_{j}=2 \text { and } E_{t}^{j}<40 \mathrm{GeV}
$$

4. opposite lepton charge signs;

5. $\not E_{t}>\frac{1}{4} m_{H}$;

6. $\not E_{t}>50 \mathrm{GeV}$ or $\Delta \phi_{\not_{t}, \ell / j}>20^{\circ}$;

7. $m_{\ell \ell}<\frac{1}{2} m_{H}-5 \mathrm{GeV}$;

8. $E_{t}^{\ell_{1}}+E_{t}^{\ell_{2}}+\not E_{t}<m_{H}$. 


\section{Chapter 8}

\section{Estimate of Signal Acceptance}

The signal acceptance was estimated by applying the event selection cuts, trigger efficiencies and lepton reconstruction and identification scale factors to Monte Carlo samples. In the following text, the leptonic decay branching fractions of the $W$ bosons were applied wherever the signal means $p \bar{p} \rightarrow H \rightarrow W^{+} W^{-}$with the $W$ bosons decaying to anything.

\subsection{Signal Monte Carlo}

The signal was modeled with the PYTHIA 6.2(16) [46] MC generator, using the CTEQ5L parton distribution function [47] and adding tune_A underlying events [48], plus the GEANT3(.21) [49] detector simulation, CDFSim and TRGSim++ of production version 5.3.3, and processed into topNt 5.3.3_nt [50] ntuples for analysis. The detector simulation took run dependence into account and the MC samples covered the analyzed data range of run number 141544-186598.

The process for generating the signal MC samples was $g g \rightarrow h^{0} \rightarrow W^{+(*)} W^{-(*)} \rightarrow$ $\ell^{+} \nu_{\ell} \ell^{-} \bar{\nu}_{\ell}$ (forced decays), where $\ell \in\{e, \mu, \tau\}$, with the Higgs boson mass at every $10 \mathrm{GeV}$ increment from $110 \mathrm{GeV}$ to $200 \mathrm{GeV}$. Gluon-gluon fusion is by far the domi- 


\begin{tabular}{|c|c|c|c|}
\hline \hline $\begin{array}{c}m_{H} \\
(\mathrm{GeV})\end{array}$ & $\begin{array}{c}\text { dataset } \\
\text { DFC ID }\end{array}$ & $\begin{array}{c}\text { number } \\
\text { of events }\end{array}$ & $\begin{array}{r}\sigma(g g \rightarrow H) \times \mathrm{BR}(H \rightarrow W W) \times \mathrm{BR}(W \rightarrow \ell \nu)^{2} \\
(\mathrm{pb})\end{array}$ \\
\hline \hline 110 & wexocw & $1,069,855$ & $0.900 \times 0.044 \times(0.3257 \pm 0.0028)^{2}$ \\
\hline 120 & wexobw & $1,063,469$ & $0.704 \times 0.132 \times(0.3257 \pm 0.0028)^{2}$ \\
\hline 130 & wexo5w & $1,127,915$ & $0.558 \times 0.287 \times(0.3257 \pm 0.0028)^{2}$ \\
\hline 140 & wexo6w & $1,074,251$ & $0.448 \times 0.483 \times(0.3257 \pm 0.0028)^{2}$ \\
\hline 150 & wexo7w & $1,089,119$ & $0.364 \times 0.681 \times(0.3257 \pm 0.0028)^{2}$ \\
\hline 160 & wexo8w & $1,051,020$ & $0.298 \times 0.901 \times(0.3257 \pm 0.0028)^{2}$ \\
\hline 170 & wexo9w & $1,033,214$ & $0.247 \times 0.965 \times(0.3257 \pm 0.0028)^{2}$ \\
\hline 180 & wexoaw & $1,079,780$ & $0.205 \times 0.935 \times(0.3257 \pm 0.0028)^{2}$ \\
\hline 190 & wexodw & $1,092,527$ & $0.172 \times 0.776 \times(0.3257 \pm 0.0028)^{2}$ \\
\hline 200 & wexoew & $1,093,662$ & $0.145 \times 0.735 \times(0.3257 \pm 0.0028)^{2}$ \\
\hline \hline
\end{tabular}

Table 8.1: Information about the signal MC samples. The NLO cross-section and branching fraction values are taken from [9] and [28].

nant process contributing to $p \bar{p} \rightarrow H$ production at the Tevatron. More informations about the signal MC samples are available in Table 8.1.

\subsection{Estimate of Acceptance}

The signal acceptance was calculated by

$$
\epsilon_{\text {acc }}=\epsilon_{\text {raw acc }} \times \epsilon_{\text {trg }} \times \mathrm{SF}_{1} \times \mathrm{SF}_{2}\left(\times \mathrm{SF}_{\mathrm{PHX} \text { QID }}\right)
$$

for each dilepton type.

\subsubsection{Raw Acceptance}

The raw signal acceptance was calculated as the number of events that passed the signal selection cuts divided by the number of total input events

$$
\epsilon_{\text {raw } a c c} \equiv \frac{N_{\text {passing event selection }}}{N_{\text {total input }}}
$$

for each dilepton type. 


\subsubsection{Correction for Trigger Efficiency}

The raw acceptance was corrected with the trigger efficiencies as shown in Table 8.2 in accordance with dilepton types. If a selected event met two trigger requirements, the equivalent trigger efficiency was calculated by

$$
\begin{aligned}
\epsilon_{t r g} & =1-\left(1-\epsilon_{t r g 1}\right) \times\left(1-\epsilon_{t r g 2}\right) \\
& =\epsilon_{t r g 1}+\epsilon_{t r g 2}-\epsilon_{t r g 1} \times \epsilon_{t r g 2}
\end{aligned}
$$

Given the fact that the signal event selection cuts were significantly more restrictive than the trigger requirements, particularly on leptons and missing transverse energy, plateau trigger efficiencies were applied to the raw acceptance, where the high $E_{t}$ central electron trigger efficiency depended on run period and SVX II participation.

\subsubsection{Correction for Lepton Reconstruction and Identification}

The raw acceptance was also corrected with the lepton reconstruction (REC) and identification (ID) scale factors as shown in Table 8.3 depending on dilepton type and energy range. Such corrections are generally needed because lepton reconstruction and identification efficiencies inevitably differ between data and MC. The corrections were made by applying the lepton REC and ID data/MC scale factors to the weight of the selected event.

\begin{tabular}{|l||c|}
\hline \hline brief trigger name & efficiency (\%) \\
\hline \hline CEM18 & $96.20 \pm 0.66$ \\
\hline MET15_PEM20 & $91.92 \pm 0.38$ \\
\hline CMUP18 & $90.78 \pm 0.47$ \\
\hline CMX18 & $96.49 \pm 0.40$ \\
\hline \hline
\end{tabular}

Table 8.2: High $p_{t}$ lepton trigger efficiencies. 


\begin{tabular}{|l||c|c|}
\hline \hline \multirow{2}{*}{\multicolumn{1}{|l||l}{$\begin{array}{l}\text { lepton } \\
\text { type }\end{array}$}} & \multicolumn{2}{c|}{ data/MC REC \& ID scale factor } \\
\cline { 2 - 3 } & $10<E_{t}<20$ & $E_{t}>20 \mathrm{GeV}$ \\
\hline \hline TCE & $1.03 \pm 0.02$ & $0.996 \pm 0.005$ \\
\hline PHX & - & $0.948 \pm 0.016$ \\
\hline CMUP & $0.85 \pm 0.05$ & $0.8921 \pm 0.0088$ \\
\hline CMX & $0.90 \pm 0.05$ & $0.9990 \pm 0.0060$ \\
\hline CMU & - & $0.8889 \pm 0.0099$ \\
\hline CMP & - & $0.9074 \pm 0.0088$ \\
\hline CMIO & - & $0.9949 \pm 0.0050$ \\
\hline \hline
\end{tabular}

Table 8.3: Lepton reconstruction and identification data/MC scale factors.

\begin{tabular}{|c||c|}
\hline \hline detector rapidity & PHX QID scale factor \\
\hline \hline $1.2 \leq\left|\eta_{\mathrm{PES}}\right| \leq 1.4$ & $1.000 \pm 0.006$ \\
\hline $1.4<\left|\eta_{\mathrm{PES}}\right| \leq 1.6$ & $0.998 \pm 0.008$ \\
\hline $1.6<\left|\eta_{\mathrm{PES}}\right| \leq 1.8$ & $0.992 \pm 0.011$ \\
\hline $1.8<\left|\eta_{\mathrm{PES}}\right| \leq 2.0$ & $0.965 \pm 0.013$ \\
\hline \hline
\end{tabular}

Table 8.4: PHX charge identification scale factors.

\subsubsection{Correction for PHX Charge Misidentification}

Electron charge misidentification (QID) was not negligible in the forward region where the PHX tracking relies on the silicon vertex detectors. After requiring opposite lepton charge signs, the PHX QID data/MC scale factors as shown in Table 8.4 were applied to the acceptance.

\subsection{Estimate of Systematic Uncertainties}

Potential sources of systematic uncertainty in the $H W W$ dilepton signal acceptance are summarized in Table 8.7 and described below. 


\subsubsection{Generator and Parton Shower Model}

The systematic uncertainty due to the choice of an MC generator, which usually fixes the choice of a parton shower model, was estimated by comparing PYTHIA and Herwig. In this analysis, Herwig counterpart MC samples were generated with a configuration most closely resembling that of the PYTHIA ones and run through the signal event selection cuts. The difference between PYTHIA and Herwig in the estimated signal acceptance was taken as the systematic uncertainty due to the choice of an MC generator.

\subsubsection{Initial State Radiation}

To estimate the uncertainty in signal acceptance due to initial state radiation (ISR), two ISR-variant PYTHIA MC $H W W$ dilepton samples for $m_{H}=160 \mathrm{GeV}$ were generated with

the $\Lambda_{\mathrm{QCD}}$ parameter used in a space-like parton shower, $\operatorname{PARP}(61)$, either double or half of the nominal value of 0.146 ;

the squared transverse momentum evolution scale factor used in $\alpha_{s}$ and parton distributions ${ }^{1}, \operatorname{PARP}(64)$, either half of or double the nominal value of 1 .

The $H W W$ signal acceptances estimated with the ISR-variant samples, as well as some information about these samples, are listed in Table 8.5. Compared with the nominal $H W W$ signal acceptance for $m_{H}=160 \mathrm{GeV}$ as shown in Table 8.8, such ISR variation resulted in $a_{-6.84}^{+1.71 \%}$ relative change in signal acceptance; the average $4.3 \%$

\footnotetext{
${ }^{1}$ For the squared energy-momentum transfer $Q^{2} \gg \Lambda_{\mathrm{QCD}}^{2}, \alpha_{s} \approx \frac{12 \pi}{\left(33-2 N_{f}\right) \ln \left(Q^{2} / \Lambda_{\mathrm{QCD}}^{2}\right)}$ where $N_{f}$ stands for the number of quark flavors.
} 


\begin{tabular}{|c|c|c|c|c|}
\hline \hline$m_{H}(\mathrm{GeV})$ & number of events & PARP(61) & PARP $(64)$ & $H W W$ acceptance (\%) \\
\hline \hline 160 & $1,025,458$ & 0.292 & 0.5 & $0.545 \pm 0.009$ (stat) \\
\hline 160 & 874,455 & 0.073 & 2.0 & $0.595 \pm 0.011$ (stat) \\
\hline \hline
\end{tabular}

Table 8.5: Information about the ISR-variant PYTHIA MC $H W W$ dilepton samples and the $H W W$ acceptance estimated with these samples.

relative change was taken as the uncertainty in the $H W W$ signal acceptance due to ISR.

\subsubsection{Parton Distribution Function}

The uncertainty in signal acceptance due to parton distribution functions (PDF) was estimated using the Hessian method [51]. Each of the up and down variations of each of the 20 orthonormal eigenvector parameters in a CTEQ6M² PDF set is implemented with the other 19 parameters unvaried in an additional CTEQ6M.\$ PDF set, where $\$$ runs from 01 to 40 . Each pair of the up and down variations represent the range of PDF behavior that is consistent with the current global data fits. Events in the PYTHIA MC $H W W$ dilepton signal sample for $m_{H}=160 \mathrm{GeV}$ were reweighed according to the ratio of CTEQ6M.\$ to CTEQ6M PDF parameter value. The changes in signal acceptance due to individual PDF parameter reweights summed to ${ }_{-3.44}^{+2.76} \%$ in quadrature, among which the change due to eigenset 15 variation was ${ }_{-3.0}^{+2.5 \%} \%$. Symmetrically, 3\% was taken as the uncertainty in the $H W W$ signal acceptance due to the PDF.

\footnotetext{
${ }^{2}$ The difference between CTEQ6M and CTEQ5L in the $H W W$ signal acceptance is $\pm 2 \%$, negligible.
} 


\subsubsection{The $\alpha_{s}$ Strong Coupling Strength}

The $H W W$ signal acceptance for two particular PDF sets, MRST72 $\left(\alpha_{s}=\right.$ $0.1175)$ and MRST75 $\left(\alpha_{s}=0.1125\right)$, were compared and $3.3 \%$ relative difference was found and taken as the uncertainty in signal acceptance introduced by $\alpha_{s}$ directly.

\subsubsection{Trigger Efficiencies}

The uncertainty in signal acceptance introduced by trigger efficiency was estimated by shifting all the trigger efficiencies, as listed in Table 8.2, up and down by their own total uncertainties and observing the changes in signal acceptance. $\mathrm{A} \pm 0.3 \%$ signal acceptance change was seen and assigned as the uncertainty due to trigger efficiency.

\subsubsection{Lepton Reconstruction and Identification}

The uncertainty in signal acceptance introduced by lepton reconstruction and identification was estimated by shifting all the lepton REC and ID data/MC scale factors, as listed in Table 8.3, up and down by their own total uncertainties and observing the changes in signal acceptance. $\mathrm{A} \pm 1.7 \%$ signal acceptance change was seen, mainly from the $10<p_{t}<20 \mathrm{GeV}$ region, and assigned as the uncertainty due to lepton reconstruction and identification.

\subsubsection{Track Isolation}

Track isolation was required in the $H W W$ search to reduce lepton fake rates. The cut on track isolation fraction was applied to all the leptons for which the COT provides tracking information. The N-1 lepton track isolation fraction distributions 
and the cut efficiencies of trk isoFrac $<0.1$ are shown in Figure 8.1.
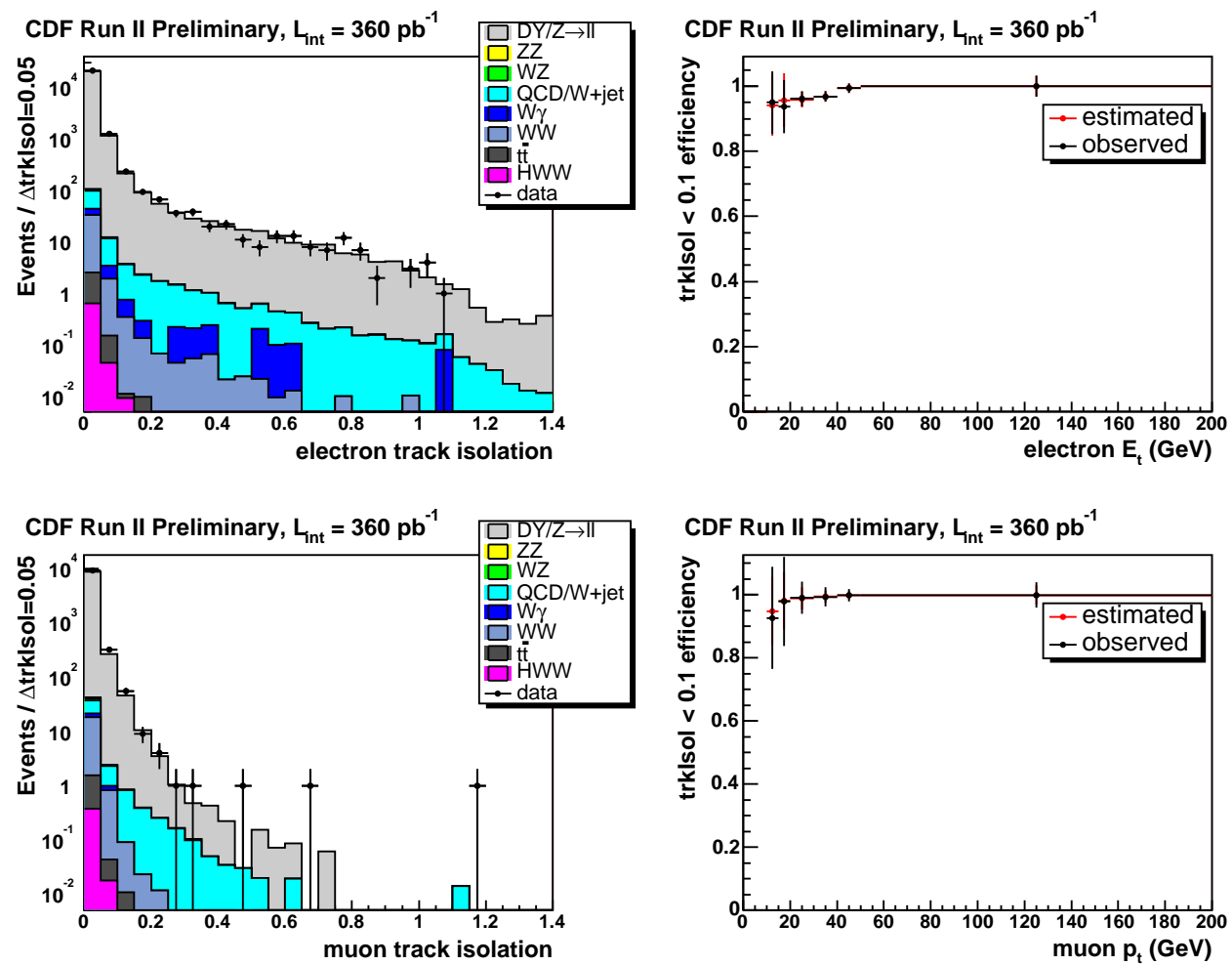

Figure 8.1: Electron (upper) and muon (lower) track isolation distributions and cut efficiencies of trk isoFrac $<0.1$ as a function of electron transverse energy or muon transverse momentum.

\begin{tabular}{|l||c|c|c|}
\hline \hline \multirow{2}{*}{\multicolumn{1}{l||}{$\begin{array}{l}\text { lepton } \\
\text { type }\end{array}$}} & \multicolumn{3}{c|}{ efficiency for the cut of track isolation fraction $<0.1$} \\
\cline { 2 - 5 } & $\frac{N_{\text {trk isoFrac }}}{N_{\text {cal } \text { iso Frac }} \text { in data }}$ & $\frac{N_{\text {trk isoFrac }}}{N_{\text {cal } \text { iso Frac }} \text { in DY MC }}$ & $\frac{\text { data }}{\text { MC }}$ scale factor \\
\hline \hline TCE & $\frac{12701}{13262}=0.9577 \pm 0.0017$ & $\frac{150772}{157404}=0.9579 \pm 0.0005$ & $0.9998 \pm 0.0019$ \\
\hline CMUP & $\frac{8378}{8430}=0.9938 \pm 0.0009$ & $\frac{122664}{123318}=0.9947 \pm 0.0002$ & $0.9991 \pm 0.0009$ \\
\hline CMX & $\frac{4701}{4738}=0.9922 \pm 0.0013$ & $\frac{66162}{66640}=0.9928 \pm 0.0003$ & $0.9994 \pm 0.0013$ \\
\hline CMU & $\frac{1113}{1119}=0.9946 \pm 0.0022$ & $\frac{16882}{16984}=0.9940 \pm 0.0006$ & $1.0006 \pm 0.0023$ \\
\hline CMP & $\frac{1327}{1336}=0.9933 \pm 0.0022$ & $\frac{21053}{21198}=0.9932 \pm 0.0006$ & $1.0001 \pm 0.0023$ \\
\hline CMIO & $\frac{1918}{1927}=0.9953 \pm 0.0016$ & $\frac{23419}{23568}=0.9937 \pm 0.0005$ & $1.0017 \pm 0.0016$ \\
\hline \hline
\end{tabular}

Table 8.6: Track isolation fraction cut efficiencies and scale factors for reference. 
Lepton track isolation was studied using $Z \rightarrow\{e e, \mu \mu\}$ events in data and MC, assuming any background was negligible. Lepton identification cuts were the same as for the $H W W$ search but without any requirement on track isolation and were restricted to the central detector region. The $Z \rightarrow\{e e, \mu \mu\}$ events were selected with the following cuts:

1. dielectron or dimuon with invariant mass $76<m_{\ell \ell}<106 \mathrm{GeV}$;

2. distance between the two leptons at the beamline, $\left|\Delta z_{0 \ell \ell}\right|<4 \mathrm{~cm}$;

3. opposite charge signs.

The cut on track isolation fraction, trk isoFrac $<0.1$, was applied to leptons in the selected $Z \rightarrow\{e e, \mu \mu\}$ events to estimate the cut efficiencies in data and MC separately and to calculate the data/MC scale factors for each lepton type. Results are shown in Table 8.6.

The uncertainty in signal acceptance introduced by the cut on track isolation fraction was estimated by shifting the track isolation scale factors up and down by their own uncertainty and observing the changes in signal acceptance. A $\pm 0.5 \%$ signal acceptance change was seen and assigned as the uncertainty due to the track isolation requirement.

\subsubsection{Jet Energy Scale}

The uncertainty in signal acceptance introduced by scaling jet energies was estimated by shifting the correction factors for jet energies up and down by their own systematic uncertainties and observing the changes in signal acceptance. $\mathrm{A}_{-0.732}^{+0.686} \%$ 
$\approx \pm 0.7 \%$ signal acceptance change was seen and assigned as the uncertainty due to the scaling of jet energies.

This analysis was not sensitive to the jet energy scale (JES) because it had a rather loose jet requirement that passed about $90 \%$ of the N-1 jet events.

\subsubsection{Total Uncertainty}

The total uncertainty in the $H W W$ signal acceptance, i.e. the sum in quadrature of the individual uncertainty items listed in Table 8.7, was about $6 \%$.

\subsection{Summary on Signal Acceptance}

A detailed table of raw and corrected $H W W$ dilepton signal acceptances through each event selection cut for each dilepton type is attached to the end of this chapter. The $p \bar{p} \rightarrow H \rightarrow W^{+} W^{-}$acceptances were calculated by applying the $W W$ dilepton decay branching fraction to the corrected total $H W W$ dilepton signal acceptances. The $H W W$ acceptances for each of the analyzed Higgs masses are listed in Table 8.8 and plotted in Figure 8.2.

\begin{tabular}{|l|c|}
\hline \hline systematic uncertainty source & uncertainty (\%) \\
\hline \hline initial state radiation & 4.3 \\
\hline$\alpha_{s}$ & 3.3 \\
\hline parton distribution function & 3 \\
\hline trigger efficiency & 0.3 \\
\hline lepton REC and ID (up to cal isoFrac) & 1.7 \\
\hline track isolation & 0.5 \\
\hline jet energy scale & 0.7 \\
\hline \hline total uncertainty & 6 \\
\hline \hline
\end{tabular}

Table 8.7: Summary of the systematic uncertainties in the $H W W$ signal acceptance. 


\section{CDF Run II Preliminary, $L_{\text {int }}=360 \mathrm{pb}^{-1}$}

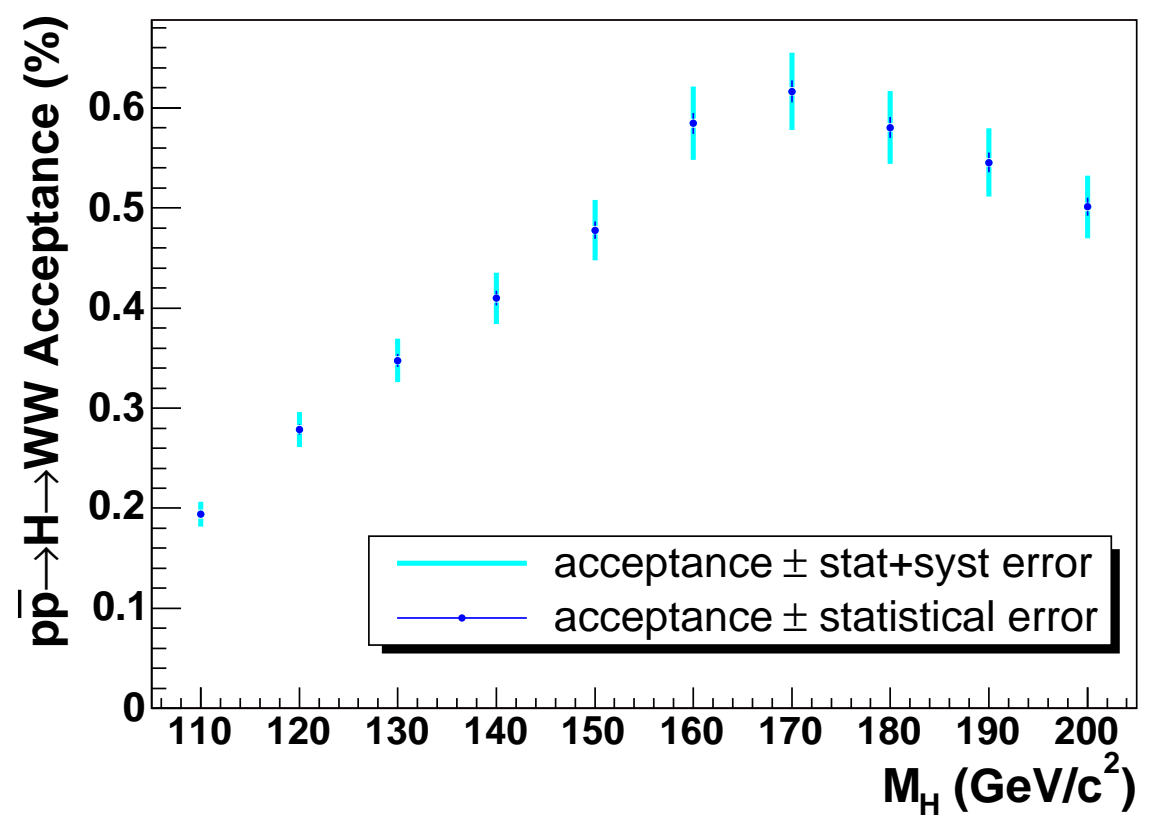

Figure 8.2: The $H W W$ signal acceptance as a function of the Higgs mass.

\begin{tabular}{|c|c|}
\hline \hline$m_{H}(\mathrm{GeV})$ & $p \bar{p} \rightarrow H \rightarrow W W$ signal acceptance (\%) \\
\hline \hline 110 & $0.194 \pm 0.004$ (stat) \pm 0.012 (syst) \\
\hline 120 & $0.279 \pm 0.005$ (stat) \pm 0.017 (syst) \\
\hline 130 & $0.348 \pm 0.006$ (stat) \pm 0.021 (syst) \\
\hline 140 & $0.410 \pm 0.007$ (stat) \pm 0.025 (syst) \\
\hline 150 & $0.478 \pm 0.009$ (stat) \pm 0.029 (syst) \\
\hline 160 & $0.585 \pm 0.010$ (stat) \pm 0.035 (syst) \\
\hline 170 & $0.617 \pm 0.011$ (stat) \pm 0.037 (syst) \\
\hline 180 & $0.580 \pm 0.010$ (stat) \pm 0.035 (syst) \\
\hline 190 & $0.546 \pm 0.010$ (stat) \pm 0.033 (syst) \\
\hline 200 & $0.501 \pm 0.009$ (stat) \pm 0.030 (syst) \\
\hline \hline
\end{tabular}

Table 8.8: The $H W W$ signal acceptance, i.e. the $\mathrm{MC}$ estimated $H W W$ dilepton signal acceptance multiplied by the $W W$ dilepton decay branching fraction, as a function of the Higgs mass. 
Raw $H W W$ dilepton signal acceptances with statistical errors (\%) for $m_{H}=160 \mathrm{GeV} \quad N_{\text {input } H W W \text { dilepton events }}=1051020$

\begin{tabular}{|c|c|c|c|c|c|c|c|c|c|c|c|}
\hline type & dilepton & cal isoFrac & trk isoFrac & $m_{\ell \ell}$ & jet veto & charge & $\not \not_{t}>m_{H} / 4$ & $\not \not_{t} \| \Delta \phi_{\not_{t} \ell / j}$ & $m_{\ell \ell} \lesssim m_{H} / 2$ & $\Sigma E_{t}^{\ell}+\not B_{t}$ & c orrect ed \\
\hline TCE- & 22638 & 18669 & 16786 & 15987 & 14051 & 14048 & 11235 & 111117 & 10871 & 10362 & 10362 \\
\hline TCE & $.15 \pm 0.01$ & $.78 \pm 0.01$ & $1.60 \pm 0.01$ & $.52 \pm 0.01$ & $.34 \pm 0.01$ & $1.34 \pm 0.01$ & $1.07 \pm 0.01$ & $1.06 \pm 0.01$ & $1.03 \pm 0.01$ & $0.99 \pm 0.01$ & $0.97 \pm 0.06$ \\
\hline TCE- & 8087 & 7379 & 6971 & 6875 & 6146 & 5664 & 4602 & 4553 & 4314 & 4238 & 4238 \\
\hline PHX & $.77 \pm 0.01$ & $0.70 \pm 0.01$ & $0.66 \pm 0.01$ & $0.65 \pm 0.01$ & $0.59 \pm 0.01$ & $0.54 \pm 0.01$ & $0.44 \pm 0.01$ & $0.43 \pm 0.01$ & $0.41 \pm 0.01$ & $0.40 \pm 0.01$ & $0.35 \pm 0.02$ \\
\hline$e e$ & $\begin{array}{c}30725 \\
2.92 \pm 0.02 \\
\end{array}$ & $\begin{array}{c}26048 \\
2.48 \pm 0.02 \\
\end{array}$ & $\begin{array}{c}23757 \\
2.26 \pm 0.01 \\
\end{array}$ & $\begin{array}{c}22862 \\
2.18 \pm 0.01\end{array}$ & $\begin{array}{c}20197 \\
1.92 \pm 0.01\end{array}$ & $\begin{array}{c}19712 \\
1.88 \pm 0.01\end{array}$ & $\begin{array}{c}15837 \\
1.51 \pm 0.01\end{array}$ & $\begin{array}{c}15670 \\
1.49 \pm 0.01\end{array}$ & $\begin{array}{c}15185 \\
1.45 \pm 0.01\end{array}$ & $\begin{array}{c}14600 \\
1.39 \pm 0.01\end{array}$ & $\begin{array}{c}14600 \\
1.32 \pm 0.08\end{array}$ \\
\hline TCE- & 2828827 & ב 23920 & 20 & ב 21200 & ב 18622 & ב 18619 & 1014768 & (214619 & 1014269 & 1013667 & 1013667 \\
\hline CMUP & $2.74 \pm 0.02$ & $2.28 \pm 0.02$ & $2.13 \pm 0.01$ & $2.02 \pm 0.01$ & $1.77 \pm 0.01$ & $1.77 \pm 0.01$ & $1.41 \pm 0.01$ & $1.39 \pm 0.01$ & $1.36 \pm 0.01$ & $1.30 \pm 0.01$ & $1.12 \pm 0.07$ \\
\hline TCE- & 12306 & 10338 & 9680 & 9296 & 8220 & 8217 & 6504 & 6446 & 6276 & 6061 & 6061 \\
\hline $\mathrm{CMX}$ & $1.17 \pm 0.01$ & $0.98 \pm 0.01$ & $0.92 \pm 0.01$ & $0.88 \pm 0.01$ & $0.78 \pm 0.01$ & $0.78 \pm 0.01$ & $0.62 \pm 0.01$ & $0.61 \pm 0.01$ & $0.60 \pm 0.01$ & $0.58 \pm 0.01$ & $0.19 \pm 0.01$ \\
\hline TCE- & 4722 & 3935 & 3703 & 3594 & 3162 & 3162 & 2630 & 2598 & 2526 & 2413 & 2413 \\
\hline $\mathrm{CMU}$ & $0.45 \pm 0.01$ & $0.37 \pm 0.01$ & $0.35 \pm 0.01$ & $0.34 \pm 0.01$ & $0.30 \pm 0.01$ & $0.30 \pm 0.01$ & $0.25 \pm 0.01$ & $0.25 \pm 0.01$ & $0.24 \pm 0.01$ & $0.23 \pm 0.01$ & $0.29 \pm 0.02$ \\
\hline TCE- & 6796 & 5736 & 5408 & 5236 & 4629 & 4629 & 3899 & 3860 & 3770 & 3598 & 3598 \\
\hline CMP & $0.65 \pm 0.01$ & $0.55 \pm 0.01$ & $0.52 \pm 0.01$ & $0.50 \pm 0.01$ & $0.44 \pm 0.01$ & $0.44 \pm 0.01$ & $0.37 \pm 0.01$ & $0.37 \pm 0.01$ & $0.36 \pm 0.01$ & $0.34 \pm 0.01$ & $0.50 \pm 0.03$ \\
\hline TCE- & 7719 & 6351 & 5931 & 5638 & 4955 & 4955 & 3867 & 3805 & 3722 & 3600 & 3600 \\
\hline CMIO & $0.73 \pm 0.01$ & $0.60 \pm 0.01$ & $0.56 \pm 0.01$ & $0.54 \pm 0.01$ & $0.47 \pm 0.01$ & $0.47 \pm 0.01$ & $0.37 \pm 0.01$ & $0.36 \pm 0.01$ & $0.35 \pm 0.01$ & $0.34 \pm 0.01$ & $0.32 \pm 0.02$ \\
\hline PHX- & 3776 & 3538 & 3478 & 3477 & 3140 & 2902 & 2403 & 2372 & 2225 & 2212 & 2212 \\
\hline CMUP & $0.36 \pm 0.01$ & $0.34 \pm 0.01$ & $0.33 \pm 0.01$ & $0.33 \pm 0.01$ & $0.30 \pm 0.01$ & $0.28 \pm 0.01$ & $0.23 \pm 0.01$ & $0.23 \pm 0.01$ & $0.21 \pm 0.004$ & $0.21 \pm 0.004$ & $0.16 \pm 0.01$ \\
\hline PHX- & 3175 & 2918 & 2871 & 2794 & 2486 & 2253 & 1837 & 1812 & 1752 & 1701 & 1701 \\
\hline CMX & $30 \pm 0.01$ & $28 \pm 0.01$ & $0.27 \pm 0.01$ & $0.27 \pm 0.01$ & $0.24 \pm 0.01$ & $0.21 \pm 0.01$ & $0.18 \pm 0.004$ & $0.17 \pm 0.004$ & $0.17 \pm 0.004$ & $0.16 \pm 0.0$ & $0.04 \pm 0.004$ \\
\hline PHX- & 865 & 822 & 815 & 813 & 723 & 670 & 583 & 580 & 550 & 541 & 541 \\
\hline $\mathrm{CMU}$ & $08 \pm 0.003$ & $08 \pm 0.003$ & $08 \pm 0.003$ & $08 \pm 0.003$ & $.07 \pm 0.003$ & $06 \pm 0.002$ & $0.06 \pm 0.002$ & $0.06 \pm 0.002$ & $0.05 \pm 0.002$ & $0.05 \pm 0.002$ & $0.04 \pm 0.003$ \\
\hline PHX- & 863 & 810 & 805 & 805 & 724 & & 591 & 582 & 542 & 542 & 542 \\
\hline CMP & $.08 \pm 0.003$ & $0.08 \pm 0.003$ & $08 \pm 0.003$ & $08 \pm 0.003$ & $.07 \pm 0.003$ & $0.06 \pm 0.002$ & $0.06 \pm 0.002$ & $0.06 \pm 0.002$ & $0.05 \pm 0.002$ & $0.05 \pm 0.002$ & $0.12 \pm 0.01$ \\
\hline PHX- & 1741 & 1606 & 1580 & 1551 & 1383 & 1277 & 1074 & 1060 & 1004 & 983 & 983 \\
\hline CMIO & $0.17 \pm 0.004$ & $0.15 \pm 0.004$ & $0.15 \pm 0.004$ & $0.15 \pm 0.004$ & $0.13 \pm 0.004$ & $0.12 \pm$ & $0.10 \pm 0.003$ & $0.10 \pm 0.003$ & $0.10 \pm 0.003$ & $0.09 \pm 0.003$ & $0.08 \pm 0.01$ \\
\hline$e \mu$ & $\begin{array}{c}70790 \\
6.74 \pm 0.02 \\
\end{array}$ & $\begin{array}{c}59974 \\
5.71 \pm 0.02 \\
\end{array}$ & $\begin{array}{c}56620 \\
5.39 \pm 0.02 \\
\end{array}$ & $\begin{array}{c}54404 \\
5.18 \pm 0.02\end{array}$ & $\begin{array}{c}48044 \\
4.57 \pm 0.02\end{array}$ & $\begin{array}{c}47356 \\
4.51 \pm 0.02\end{array}$ & $\begin{array}{c}38156 \\
3.63 \pm 0.02\end{array}$ & $\begin{array}{c}37734 \\
3.59 \pm 0.02\end{array}$ & $\begin{array}{c}36636 \\
3.49 \pm 0.02\end{array}$ & $\begin{array}{c}35318 \\
3.36 \pm 0.02\end{array}$ & $\begin{array}{c}35318 \\
2.85 \pm 0.17\end{array}$ \\
\hline$\overline{\text { CMUP- }}$ & 99553 & $\begin{array}{l}8390 \\
\end{array}$ & 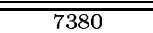 & (26932 & (6035 & " 6035 & 47744 & " 4691 & $=4583$ & " 4389 & " 4389 \\
\hline CMUP & $91 \pm 0.01$ & $80 \pm 0.01$ & $70 \pm 0.01$ & $66 \pm 0.01$ & $57 \pm 0.01$ & $57 \pm 0.01$ & $45 \pm 0.01$ & $45 \pm 0.01$ & $0.44 \pm 0.01$ & $0.42 \pm 0.01$ & $0.33 \pm 0.02$ \\
\hline CMUP- & 7410 & 6765 & 6467 & 6262 & 5533 & 5533 & 4432 & 4379 & 4266 & 4181 & 4181 \\
\hline CMX & $71 \pm 0.01$ & $64 \pm$ & $62 \pm 0.01$ & $60 \pm 0.01$ & $53 \pm 0.01$ & $53 \pm$ & $42 \pm 0.01$ & $.42 \pm 0.01$ & $.41 \pm 0.01$ & $0.40 \pm 0.01$ & $0.11 \pm 0.01$ \\
\hline CMUP & 3022 & 275 & 2538 & 2475 & 2150 & 21 & 1743 & 1729 & 1688 & 1636 & 1636 \\
\hline $\mathrm{CMU}$ & $29 \pm 0$. & $26 \pm$ & $24 \pm 0.01$ & $24 \pm 0.01$ & $.21 \pm 0.004$ & $0.21 \pm$ & $.17 \pm 0.004$ & $0.17 \pm 0.004$ & $0.16 \pm 0.004$ & $0.16 \pm 0.004$ & $0.17 \pm 0.01$ \\
\hline CMUP- & 4826 & 438 & 3796 & 3649 & 3201 & & 2626 & 2599 & 254 & 2453 & 2453 \\
\hline CMP & $46 \pm 0.01$ & $42 \pm$ & $36 \pm 0.01$ & $35 \pm 0.01$ & $31 \pm$ & $31 \pm$ & $0.25 \pm 0.01$ & $25 \pm$ & $0.24 \pm 0.01$ & $0.23 \pm 0.01$ & $0.31 \pm 0.02$ \\
\hline CMUP- & 4973 & $\overline{442}$ & 4129 & 3917 & 3455 & 345 & 2696 & 2667 & 2608 & 2529 & 2529 \\
\hline CMIO & $47 \pm 0.01$ & $42 \pm 0.01$ & $39 \pm 0.01$ & $37 \pm 0.01$ & $33 \pm 0.01$ & $33 \pm 0.01$ & $0.26 \pm 0.01$ & $0.25 \pm 0.01$ & $0.25 \pm 0.01$ & $0.24 \pm 0.01$ & $0.20 \pm 0.01$ \\
\hline CMX- & 1738 & 1498 & 1177 & 1104 & 980 & 980 & 727 & 719 & 699 & 675 & 675 \\
\hline $\mathrm{CM}$ & $17 \pm 0.0$ & $4 \pm 0.0$ & $1 \pm 0.0$ & $11 \pm 0.003$ & $09 \pm 0.003$ & $09 \pm$ & $07 \pm 0.003$ & $07 \pm 0.003$ & $07 \pm 0.003$ & $0.06 \pm 0.002$ & $0.06 \pm 0.004$ \\
\hline CMX- & 979 & 882 & 783 & 743 & 675 & 675 & 553 & 547 & 529 & 510 & 510 \\
\hline $\mathrm{CMU}$ & $09 \pm 0.003$ & $8 \pm 0.003$ & $7 \pm 0.003$ & $07 \pm 0.003$ & $06 \pm 0.002$ & $06 \pm 0.002$ & $.05 \pm 0.002$ & $0.05 \pm 0.002$ & $0.05 \pm 0.002$ & $0.05 \pm 0.06$ & $0.04 \pm 0.003$ \\
\hline CMX- & 1443 & 135 & 1308 & 1293 & 1118 & 1118 & 928 & 920 & 900 & 881 & 881 \\
\hline CMP & $14 \pm 0.0$ & $3 \pm 0.0$ & $12 \pm 0.0$ & $12 \pm 0.0$ & $11 \pm 0.0$ & $11 \pm$ & $0.09 \pm 0.003$ & $09 \pm 0.003$ & $0.09 \pm 0.003$ & $0.08 \pm 0.003$ & $0.07 \pm 0.01$ \\
\hline CMX- & 1619 & 1459 & 1321 & 1270 & 1119 & 111 & 834 & 826 & 795 & 772 & 772 \\
\hline CMIO & $15 \pm 0$ & $14 \pm$ & $13 \pm 0$ & $12 \pm 0.003$ & $11 \pm 0$ & $0.11 \pm$ & $0.08 \pm 0$ & $0.08 \pm 0.003$ & $0.08 \pm 0.003$ & $0.07 \pm 0.003$ & $0.06 \pm 0.01$ \\
\hline$\mu \mu$ & $\begin{array}{c}35563 \\
3.38 \pm 0.02\end{array}$ & $\begin{array}{c}31914 \\
3.04 \pm 0.02\end{array}$ & $\begin{array}{c}28899 \\
2.75 \pm 0.02\end{array}$ & $\begin{array}{c}27645 \\
2.63 \pm 0.02\end{array}$ & $\begin{array}{c}24266 \\
2.31 \pm 0.02\end{array}$ & $\begin{array}{c}24266 \\
2.31 \pm 0.02\end{array}$ & $\begin{array}{c}19283 \\
1.84 \pm 0.01\end{array}$ & $\begin{array}{c}19077 \\
1.82 \pm 0.01\end{array}$ & $\begin{array}{c}18615 \\
1.77 \pm 0.01\end{array}$ & $\begin{array}{c}18026 \\
1.72 \pm 0.01\end{array}$ & $\begin{array}{c}18026 \\
1.35 \pm 0.08\end{array}$ \\
\hline total & $\begin{array}{c}137078 \\
13.04 \pm 0.03\end{array}$ & $\begin{array}{c}117936 \\
11.22 \pm 0.03\end{array}$ & $\begin{array}{c}109276 \\
10.40 \pm 0.03\end{array}$ & $\begin{array}{c}104911 \\
9.98 \pm 0.03\end{array}$ & $\begin{array}{c}92507 \\
8.80 \pm 0.03\end{array}$ & $\begin{array}{c}91334 \\
8.69 \pm 0.03\end{array}$ & $\begin{array}{c}73276 \\
6.97 \pm 0.03\end{array}$ & $\begin{array}{c}72481 \\
6.90 \pm 0.03\end{array}$ & $\begin{array}{c}70436 \\
6.70 \pm 0.02\end{array}$ & $\begin{array}{c}67944 \\
6.47 \pm 0.02\end{array}$ & $\begin{array}{c}67944 \\
5.51 \pm 0.33\end{array}$ \\
\hline
\end{tabular}




\section{Chapter 9}

\section{Estimate of Background}

Events with two leptons plus large missing transverse energy could be produced by the $H W W$ dilepton process, although it is far more likely that such events are produced by other SM processes instead, in which case these events are the background.

\subsection{Background Sources}

The SM backgrounds mainly came from the following sources.

\subsection{1 $W W$}

Because the final state objects were exactly the same as the signal and the crosssection was not small, the SM continuum $p \bar{p} \rightarrow W^{+} W^{-}$production when both on-shell $W$ bosons decay leptonically contributed the majority of the background ${ }^{1}$, especially at high masses where the $W$ bosons from the Higgs boson tended to be both on-shell and the spin constraint imposed by the Higgs boson was the sole signal discriminator.

\footnotetext{
${ }^{1}$ Other $W W$ decay modes contributed negligibly little, such as $W W \rightarrow \ell \nu q q$ with one jet faking a lepton and the other jet with $E_{t}<55 \mathrm{GeV}$ or even $W W \rightarrow q q q q$ with two jets faking leptons, the other two jets both with $E_{t}<40 \mathrm{GeV}$ and instrumental $\not f_{t}$.
} 
The continuum $W W$ production constituted $36 \%-72 \%$ of the total background, as shown in Table 9.3-9.12.

\subsection{2 $t \bar{t}$}

The SM $t \bar{t}$ production contributed $0.8 \%-4.4 \%$ of the total background, primarily through the dilepton decay channel of $p \bar{p} \rightarrow t \bar{t} \rightarrow W^{+} W^{-} b \bar{b} \rightarrow \ell^{+} \nu \ell^{-} \bar{\nu} b \bar{b}$. With two $b$-jets in the final state, much of the $t \bar{t}$ background was vetoed by the jet requirement.

\subsection{3 $Z Z$}

The SM $Z Z$ production contributed $0.2 \%-2.3 \%$ of the total background, mainly from the $\ell \ell \nu \nu$ final state. The dilepton invariant mass cut, $m_{\ell \ell}<\frac{1}{2} m_{H}-5 \mathrm{GeV}$, suppressed this background for $m_{H} \lesssim 160 \mathrm{GeV}$.

\subsection{4 $W Z$}

The SM $W Z$ production contributed up to $7 \%$ of the total background, mostly due to the misidentification of one of the leptons in the $\ell \nu \ell \ell$ final state or the occurrence

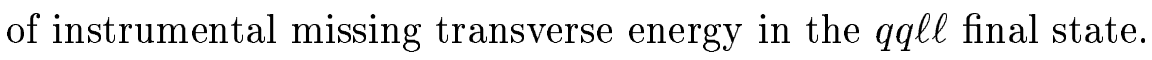

\subsubsection{Drell-Yan}

Due to the occurrence of instrumental $\mathbb{E}_{t}$, the Drell-Yan (DY) dilepton $p \bar{p} \rightarrow$ $Z / \gamma^{*} \rightarrow \ell^{+} \ell^{-}$production contributed $5.5 \%-20 \%$ of the total background. The crosssection for DY production was large and the gigantic $m_{Z}$ peak entered the background at high masses; however, with the rapid decrease of the DY $E_{t}$ distribution as $E_{t}$ increased, the cut $E_{t}>\frac{1}{4} m_{H}$ effectively reduced the DY background.

The charged lepton pair that are daughters of $Z / \gamma^{*}$ in the $W Z, Z Z$ or DY pro- 
ductions tend to be back-to-back with a large dilepton azimuthal angle, in contrast to the leptons from the $H W W$ decay. Because this background contributed to large- $\Delta \phi_{\ell \ell}$ bins that had little significance in signal cross-section limit extraction, it was expected and indeed observed that $Z / \gamma^{*}$ dilepton associated background had little impact on the $H W W$ production cross-section limits extracted from dilepton azimuthal angle distributions.

\subsection{6 $W \gamma$}

The SM $p \bar{p} \rightarrow W \gamma \rightarrow \ell \nu \gamma$ process contributed to the background when the photon was accidentally matched to a track and misidentified as an electron of either charge sign. The $W \gamma$ process contributed $3.6 \%-27 \%$ of the total background, $e-\mu$ asymmetrically. The requirement on calorimetric and track isolation and the requirement of opposite charge signs each suppressed the $W \gamma$ background by half. The upper $m_{\ell \ell}$ cut removed about $40 \%$ of the $W \gamma$ background. Furthermore, because the missing transverse energy from $W \gamma$ populated the region of $20<\boldsymbol{E}_{t}<60 \mathrm{GeV}$, the $W \gamma$ background decreased dramatically as $m_{H}$ increased and the $\not_{t}$ cut became more restrictive.

\subsubsection{QCD $/ W+$ jet}

The $p \bar{p} \rightarrow W+$ jet $\rightarrow \ell \nu+$ jet process contributed to the background when one jet was misidentified as an electron or a muon; pure QCD multi-jet production contributed to the background when two jets were misidentified as leptons and instrumental $\not_{t}$ occurred. Since the probability of a jet faking a lepton was small, as shown in Chapter 6, pure QCD background was smaller than the $W+$ jet background 
by two orders of magnitude. The two processes contributed 5.5\%-15\% of the total background. The effect of the selection cuts on the QCD $/ W+$ jet background was similar to the effects on the $W \gamma$ background. The likelihood of misidentifying a jet as a lepton was suppressed by the requirement on track isolation.

A cross-check with the same-sign dilepton events verified the PHX charge misidentification rate, $W \gamma \mathrm{MC}$ modeling and the rates at which jets faked leptons in this analysis since these instrumental defects contributed a statistically sensible amount of same-sign dilepton events in the analyzed high $p_{t}$ lepton datasets. The $E_{t}^{\ell_{1}}+E_{t}^{\ell_{2}}+Z_{t}$ distributions of same-sign dilepton events for $m_{H}=160 \mathrm{GeV}$, as shown in Figure 9.1, was plotted after requiring events to have two leptons with the trigger lepton

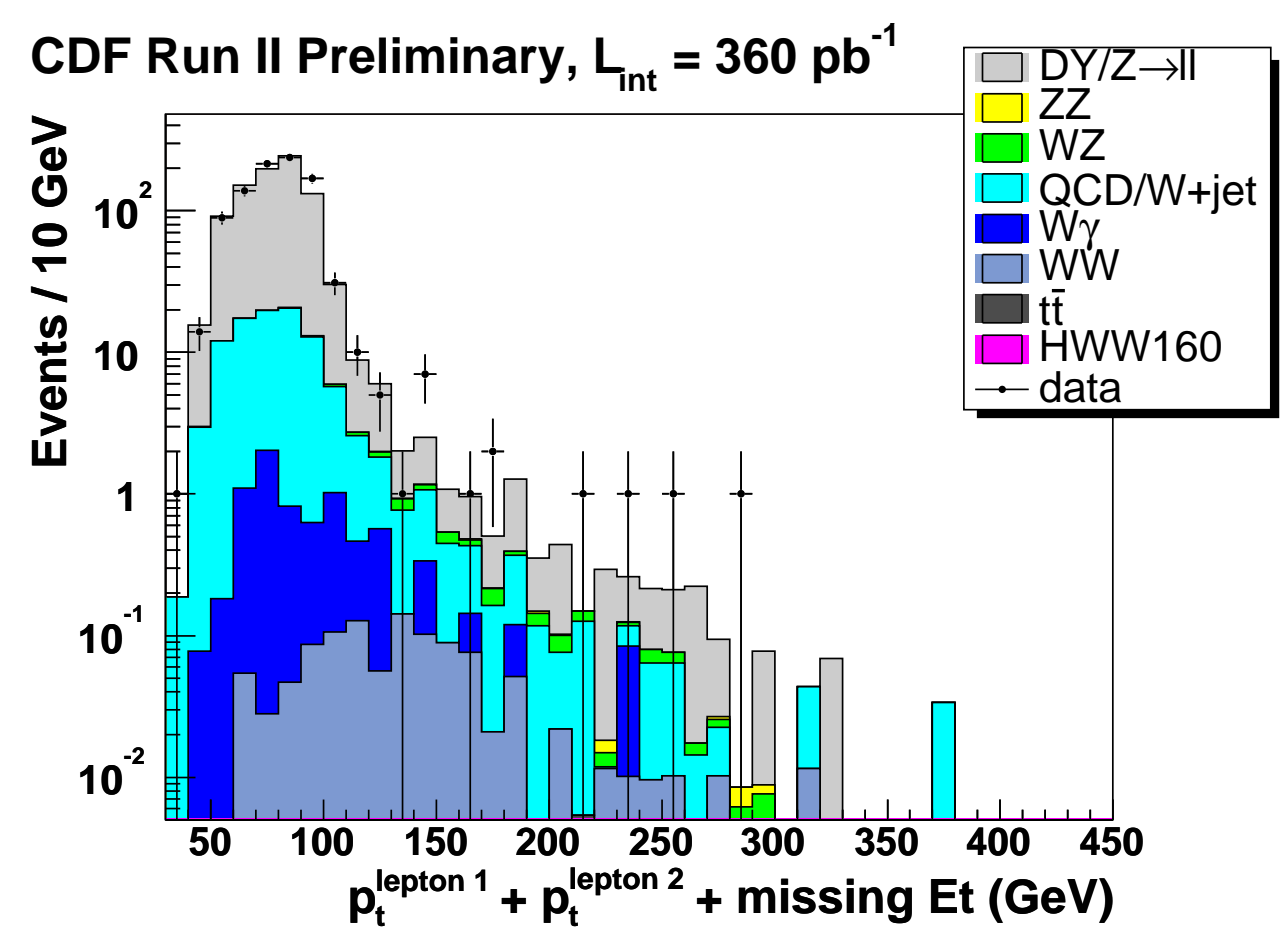

Figure 9.1: Initial $E_{t}^{\ell_{1}}+E_{t}^{\ell_{2}}+\not{ }_{t}$ distributions of same-sign dilepton events for $m_{H}=$ $160 \mathrm{GeV}$. 
$E_{t}>20 \mathrm{GeV}$ and the non-trigger lepton $E_{t}>10 \mathrm{GeV}$ and a dilepton invariant mass

of $m_{\ell \ell}>16 \mathrm{GeV}$. Good agreement was observed, demonstrating the capabilities of CDFSim and our understanding of these instrumental defects.

\subsection{Estimate Procedure}

Backgrounds that are simulated well by $\mathrm{MC}$ can be estimated reliably by $\mathrm{MC}$ and were therefore estimated by $\mathrm{MC}$. Only the $\mathrm{QCD} / W+$ jet background could not be estimated by MC because the PYTHIA parton shower simulation tended to underestimate the isolation condition of jet and would result in an overestimation of this background; hence, the $\mathrm{QCD} / W+$ jet background was estimated with data from jet triggers.

\subsubsection{Monte Carlo Approach}

The SM diboson $(W W, W \gamma, W Z$ and $Z Z), t \bar{t}$ and Drell-Yan dilepton backgrounds were estimated by MC. Most of the SM backgrounds were modeled with the PYTHIA 6.2(16) MC generator, using the CTEQ5L parton distribution function and adding tune_A underlying events, including

$W W$ - inclusive production and decay (at the Tevatron Run II) with a theoretical next-to-leading order cross-section of $\sigma_{\mathrm{NLO}}\left(p \bar{p} \rightarrow W^{+} W^{-}\right)=12.4 \pm 0.8 \mathrm{pb}$ at $\sqrt{s}=1.96 \mathrm{TeV}[53], 0.4 \mathrm{M}$ events;

$W Z$ - inclusive production and decay with $\sigma_{\mathrm{NLO}}(p \bar{p} \rightarrow W Z)=3.65 \pm 0.26 \mathrm{pb}$ at $\sqrt{s}=1.96 \mathrm{TeV}[54], 0.4 \mathrm{M}$ events;

$Z Z$ - inclusive production and decay with $\sigma_{\mathrm{NLO}}(p \bar{p} \rightarrow Z Z)=1.39 \pm 0.10 \mathrm{pb}$ at 
$\sqrt{s}=1.96 \mathrm{TeV}[54], 0.4 \mathrm{M}$ events;

$t \bar{t}$ - inclusive production and decay with top quark mass $m_{t}=178 \mathrm{GeV}$ and $\sigma_{\mathrm{NLO}}(p \bar{p} \rightarrow t \bar{t})=6.70 \pm 0.45 \mathrm{pb}$ at $\sqrt{s}=1.96 \mathrm{TeV}[55], 1 \mathrm{M}$ events

Drell-Yan - inclusive production and dilepton decay of $Z / \gamma^{*}$ with $m_{Z / \gamma^{*}}>$ $5 \mathrm{GeV}$, after the application of a HEPG filter with an efficiency of $\epsilon_{f l t}=2.9 \%$ that pre-selected trilepton events with electron $p_{t}>3.6 \mathrm{GeV}$ or muon $p_{t}>$ 4.8 GeV and lepton $|\eta|<1.4$. The leading-order cross-section after the filter, $\sigma_{\text {PYTHIA LO }}\left(p \bar{p} \rightarrow Z / \gamma^{*} \rightarrow \ell^{+} \ell^{-}, m_{Z / \gamma^{*}}>5 \mathrm{GeV}\right) \approx 17,354 \mathrm{pb}$ at $\sqrt{s}=1.96 \mathrm{TeV}$ where $\ell \in\{e, \mu, \tau\}$, was corrected with an NNLO/LO factor of $k=1.38$ [56]. The sample contained $3 \mathrm{M}$ events.

The $W \gamma \rightarrow \ell \nu \gamma$ background was modeled using Baur and Berger's WGAMMA [57] HEPG events recycled from a previous CDF II $W \gamma$ cross-section measurement [58]. The events were generated with photon $E_{t}>5 \mathrm{GeV}$, lepton and photon $\left|\eta_{\ell / \gamma}\right|<10$ and $\Delta R_{\ell, \gamma}>0.2$ and interfaced with the PYTHIA MC generator for parton shower, hadronization and underlying event modeling. An overall NLO/LO correction factor of $k=1.38$ was applied to obtain an NLO cross-section of $\sigma_{\mathrm{NLO}}(p \bar{p} \rightarrow W \gamma \rightarrow \ell \nu \gamma)=$ $89.4 \mathrm{pb}$ with $2 \%$ error where $\ell \in\{e, \mu\}$ [58]. The samples contained $0.35 \mathrm{M}$ events in total.

All the background MC samples went through GEANT3(.21) detector simulation, CDFSim and TRGSim++ of production version 5.3.3, and were processed into topNt 5.3.3_nt ntuples for analysis. The detector simulation was run-dependent and the samples covered the analyzed data run range. More informations about the SM background MC samples are available in Table 9.1. 


\begin{tabular}{|c|c|c|c|c|}
\hline \hline process & DFC ID & num evt & cross-section $(\mathrm{pb})$ & miscellaneous use \\
\hline \hline$W W$ & wtop1w & 392,932 & $12.4 \pm 0.8$ & \\
\hline$W Z$ & wtop1z & 381,605 & $3.65 \pm 0.26$ & \\
\hline$Z Z$ & ztopcz & 372,337 & $1.39 \pm 0.10$ & \\
\hline$t \bar{t}$ & ttopel & $1,069,877$ & $6.70 \pm 0.45$ & $m_{t}=178 \mathrm{GeV}$ \\
\hline$Z / \gamma^{*}$ & sexo8t & $2,965,259$ & $\sigma_{\mathrm{LO}} \times k \times \epsilon_{f l t} \approx 17,354 \times 1.38 \times 0.029$ \\
\hline$W \gamma$ & ktopXx & 341,257 & $89.4 \pm 1.8$ & $\mathrm{Xx} \in\{2,3\}\{e, m\}$ \\
\hline \hline
\end{tabular}

Table 9.1: Information about the background MC samples.

In the same way as of the signal, the event yield of each background was calculated by

$$
N_{\text {evt }}=\epsilon_{\text {acc }} \times \sigma \times \mathcal{L}_{\text {int }}
$$

where the factors were found as follow:

each background acceptance $\epsilon_{\text {acc }}$ was calculated in the same way as described in Section 8.2 with the "signal" being a background process. For each dilepton type, each raw background acceptance was obtained by running large background MC samples through the signal event selection cuts and dividing the number of output events by the number of total input events. The result was corrected with the trigger efficiencies and the lepton REC and ID (and PHX QID if applicable) scale factors;

the cross-section $\sigma$ for the process in question. It could be a product of the production cross-section of resonance in $p \bar{p}$ collisions at $\sqrt{s}=1.96 \mathrm{TeV}$ where NLO or a better theoretical prediction was preferred if available, the loop-correcting $k$-factor if applicable, any subsequent decay branching fraction and any HEPG filter efficiency, i.e.

$$
\sigma(\square)=\sigma_{\mathrm{NLO}}(p \bar{p} \rightarrow \square) \times \mathrm{BR}(\square \rightarrow \diamond \diamond) \times \epsilon_{f l t}
$$


where $\sigma_{\mathrm{NLO}}(p \bar{p} \rightarrow \square)$ could be $k_{\mathrm{NLO} / \mathrm{LO}} \times \sigma_{\mathrm{LO}}(p \bar{p} \rightarrow \square)$ and $\square$ and $\diamond \diamond$ stand for any particles that complete the process;

the integrated luminosity $\mathcal{L}_{\text {int }}$ corresponding to the dataset in which the signal was sought, as described in Section 4.3. It varies with dilepton type.

\subsubsection{Data Approach}

The QCD $/ W+$ jet background was estimated using jet data and high $p_{t}$ lepton data, as described in Chapter 6 .

\subsection{Systematic Uncertainties in Background}

The uncertainties in the SM $W W$, the WGAMMA-unrelated part of $W \gamma, W Z$, $Z Z, t \bar{t}$ and DY dilepton backgrounds were estimated the same way as the uncertainties in the $H W W$ signal acceptance, since all were PYTHIA MC; Section 8.3 is referred to for the methods. The uncertainties due to the WGAMMA MC generator were taken from the analysis in which the samples were generated [58]. The total uncertainty in the $\mathrm{QCD} / W+$ jet background was dominated by the dependence on the jet trigger from which data were analyzed to get lepton fake rates, as described in Section 6.1.2. The systematic uncertainties in each background are summarized in Table 9.2.

\subsection{Signal and Background Event Yields}

The signal and background event yields along with errors separately in the ee, $e \mu$ and $\mu \mu$ channels are summarized in Table 9.3-9.12, where the errors are the quadratic

sums of statistical, systematic and luminosity uncertainties since these uncertainty 


\begin{tabular}{|c|c|c|}
\hline background & uncertainty source & uncertainty \\
\hline $\bar{~} \overline{W W}$ & $\begin{array}{l}\text { MC generator and parton shower model } \\
\text { PDF } \\
\text { ISR } \\
\text { jet energy scale } \\
\text { trigger efficiency } \\
\text { lepton reconstruction and identification } \\
\text { lepton track isolation }\end{array}$ & $\begin{array}{l}4 \% \\
5 \% \\
6 \% \\
1 \% \\
1 \% \\
2 \% \\
2 \%\end{array}$ \\
\hline & total & $9 \%$ \\
\hline$W Z$ & total (assumed the same as $W W$ ) & $9 \%$ \\
\hline$Z Z$ & total (assumed the same as $W W$ ) & $9 \%$ \\
\hline$\overline{t \bar{t}}$ & $\begin{array}{l}\text { MC generator and parton shower model } \\
\text { PDF } \\
\text { ISR/FSR } \\
\text { jet energy scale } \\
\text { trigger efficiency } \\
\text { lepton reconstruction and identification } \\
\text { lepton track isolation }\end{array}$ & $\begin{array}{l}4 \% \\
1 \% \\
2 \% \\
8 \% \\
1 \% \\
2 \% \\
2 \%\end{array}$ \\
\hline & total & $10 \%$ \\
\hline Drell-Yan & $\begin{array}{l}\text { MC generator and parton shower model } \\
\text { PDF } \\
\text { ISR } \\
\text { jet energy scale } \\
\text { trigger efficiency } \\
\text { lepton reconstruction and identification } \\
\text { lepton track isolation }\end{array}$ & $\begin{array}{r}4 \% \\
2 \% \\
6 \% \\
14 \% \\
1 \% \\
2 \% \\
2 \%\end{array}$ \\
\hline & total & $16 \%$ \\
\hline$\overline{W \gamma}$ & $\begin{array}{l}\text { factorization scale } \\
\text { renormalization scale } \\
\text { PDF } \\
\text { jet energy scale } \\
\text { trigger efficiency } \\
\text { lepton reconstruction and identification } \\
\text { lepton track isolation }\end{array}$ & $\begin{array}{l}2 \% \\
3 \% \\
5 \% \\
1 \% \\
1 \% \\
2 \% \\
2 \%\end{array}$ \\
\hline & total & $7 \%$ \\
\hline $\mathrm{QCD} / W+$ jet & dependence on jet data sample & $50 \%$ \\
\hline
\end{tabular}

Table 9.2: Summary of the systematic uncertainties in the backgrounds. 
items are not correlated with one another, i.e.

$$
\delta_{\text {total }}=\sqrt{\delta_{\text {stat }}^{2}+\delta_{\text {syst }}^{2}+\delta_{\text {lumi }}^{2}}
$$

Good agreement between data and the background expectations was observed for all the analyzed Higgs masses.

\begin{tabular}{r||ccc|c}
\hline \hline$m_{H}=110 \mathrm{GeV}$ & $e e$ & $e \mu$ & $\mu \mu$ & $\ell \ell$ \\
\hline \hline$H W W$ & $0.007 \pm 0.000$ & $0.014 \pm 0.001$ & $0.007 \pm 0.000$ & $0.028 \pm 0.003$ \\
\hline$W W$ & $0.893 \pm 0.108$ & $2.271 \pm 0.273$ & $0.674 \pm 0.081$ & $3.838 \pm 0.462$ \\
$W Z$ & $0.033 \pm 0.004$ & $0.078 \pm 0.011$ & $0.034 \pm 0.004$ & $0.144 \pm 0.021$ \\
$Z Z$ & $0.006 \pm 0.001$ & $0.007 \pm 0.001$ & $0.010 \pm 0.002$ & $0.023 \pm 0.004$ \\
$t \bar{t}$ & $0.027 \pm 0.003$ & $0.027 \pm 0.003$ & $0.026 \pm 0.003$ & $0.080 \pm 0.010$ \\
$W \gamma$ & $1.909 \pm 0.180$ & $0.929 \pm 0.087$ & $0.000 \pm 0.000$ & $2.837 \pm 0.268$ \\
$D Y \ell \ell$ & $0.915 \pm 0.235$ & $0.805 \pm 0.207$ & $0.394 \pm 0.102$ & $2.114 \pm 0.544$ \\
$\mathrm{QCD} / W+$ jet & $0.564 \pm 0.284$ & $0.789 \pm 0.398$ & $0.241 \pm 0.122$ & $1.595 \pm 0.803$ \\
\hline total background & $4.348 \pm 0.476$ & $4.905 \pm 0.585$ & $1.380 \pm 0.189$ & $10.632 \pm 1.231$ \\
\hline \hline data & 3 & 3 & 2 & 8 \\
\hline \hline
\end{tabular}

Table 9.3: Summary of the signal and background event yields for $m_{H}=110 \mathrm{GeV}$.

\begin{tabular}{r||ccc|c}
\hline \hline$m_{H}=120 \mathrm{GeV}$ & $e e$ & $e \mu$ & $\mu \mu$ & $\ell \ell$ \\
\hline \hline$H W W$ & $0.025 \pm 0.002$ & $0.048 \pm 0.004$ & $0.022 \pm 0.002$ & $0.095 \pm 0.008$ \\
$W W$ & $1.330 \pm 0.160$ & $2.995 \pm 0.360$ & $1.166 \pm 0.140$ & $5.491 \pm 0.661$ \\
$W Z$ & $0.068 \pm 0.010$ & $0.112 \pm 0.016$ & $0.050 \pm 0.007$ & $0.230 \pm 0.033$ \\
$Z Z$ & $0.006 \pm 0.001$ & $0.008 \pm 0.001$ & $0.011 \pm 0.002$ & $0.025 \pm 0.005$ \\
$t \bar{t}$ & $0.036 \pm 0.005$ & $0.046 \pm 0.006$ & $0.036 \pm 0.005$ & $0.118 \pm 0.015$ \\
$W \gamma$ & $1.972 \pm 0.186$ & $0.923 \pm 0.087$ & $0.000 \pm 0.000$ & $2.895 \pm 0.273$ \\
DY $\ell \ell$ & $0.695 \pm 0.179$ & $0.538 \pm 0.138$ & $0.402 \pm 0.104$ & $1.635 \pm 0.420$ \\
QCD $/ W+$ jet & $0.635 \pm 0.320$ & $0.572 \pm 0.288$ & $0.465 \pm 0.234$ & $1.672 \pm 0.842$ \\
\hline total background & $4.743 \pm 0.500$ & $5.194 \pm 0.545$ & $2.129 \pm 0.308$ & $12.066 \pm 1.324$ \\
\hline \hline data & 2 & 4 & 1 & 7 \\
\hline \hline
\end{tabular}

Table 9.4: Summary of the signal and background event yields for $m_{H}=120 \mathrm{GeV}$. 


\begin{tabular}{r||ccc|c}
\hline \hline$m_{H}=130 \mathrm{GeV}$ & $e e$ & $e \mu$ & $\mu \mu$ & $\ell \ell$ \\
\hline \hline$H W W$ & $0.052 \pm 0.004$ & $0.105 \pm 0.009$ & $0.047 \pm 0.004$ & $0.205 \pm 0.017$ \\
\hline$W W$ & $1.689 \pm 0.203$ & $3.661 \pm 0.441$ & $1.477 \pm 0.178$ & $6.826 \pm 0.822$ \\
$Z Z$ & $0.074 \pm 0.011$ & $0.139 \pm 0.020$ & $0.072 \pm 0.010$ & $0.284 \pm 0.041$ \\
$Z Z$ & $0.006 \pm 0.001$ & $0.010 \pm 0.002$ & $0.012 \pm 0.002$ & $0.029 \pm 0.005$ \\
$t \bar{t}$ & $0.047 \pm 0.006$ & $0.060 \pm 0.008$ & $0.045 \pm 0.006$ & $0.152 \pm 0.019$ \\
$W \gamma$ & $1.294 \pm 0.122$ & $0.923 \pm 0.087$ & $0.000 \pm 0.000$ & $2.217 \pm 0.209$ \\
$D Y \ell \ell$ & $0.547 \pm 0.141$ & $0.312 \pm 0.080$ & $0.407 \pm 0.105$ & $1.265 \pm 0.325$ \\
QCD $W+$ jet & $0.682 \pm 0.343$ & $0.653 \pm 0.328$ & $0.445 \pm 0.224$ & $1.780 \pm 0.896$ \\
\hline total background & $4.339 \pm 0.491$ & $5.758 \pm 0.617$ & $2.458 \pm 0.324$ & $12.554 \pm 1.408$ \\
\hline \hline data & 2 & 6 & 1 & 9 \\
\hline \hline
\end{tabular}

Table 9.5: Summary of the signal and background event yields for $m_{H}=130 \mathrm{GeV}$.

\begin{tabular}{r||ccc|c}
\hline \hline$m_{H}=140 \mathrm{GeV}$ & $e e$ & $e \mu$ & $\mu \mu$ & $\ell \ell$ \\
\hline \hline$H W W$ & $0.081 \pm 0.007$ & $0.168 \pm 0.014$ & $0.078 \pm 0.007$ & $0.326 \pm 0.028$ \\
$W W$ & $1.942 \pm 0.234$ & $4.220 \pm 0.508$ & $1.821 \pm 0.219$ & $7.983 \pm 0.961$ \\
$Z Z$ & $0.090 \pm 0.013$ & $0.163 \pm 0.023$ & $0.084 \pm 0.012$ & $0.338 \pm 0.048$ \\
$t \bar{t}$ & $0.010 \pm 0.002$ & $0.010 \pm 0.002$ & $0.012 \pm 0.002$ & $0.032 \pm 0.006$ \\
$W \gamma$ & $0.063 \pm 0.008$ & $0.090 \pm 0.011$ & $0.058 \pm 0.007$ & $0.210 \pm 0.026$ \\
$\mathrm{DY} \ell \ell$ & $0.127 \pm 0.106$ & $0.841 \pm 0.079$ & $0.000 \pm 0.000$ & $1.967 \pm 0.186$ \\
$\mathrm{QCD} / W+$ jet & $0.655 \pm 0.330$ & $0.595 \pm 0.299$ & $0.276 \pm 0.139$ & $1.526 \pm 0.768$ \\
\hline total background & $4.199 \pm 0.472$ & $6.166 \pm 0.652$ & $2.707 \pm 0.307$ & $13.071 \pm 1.403$ \\
\hline \hline data & 4 & 6 & 4 & 14 \\
\hline \hline
\end{tabular}

Table 9.6: Summary of the signal and background event yields for $m_{H}=140 \mathrm{GeV}$.

\begin{tabular}{r||ccc|c}
\hline \hline$m_{H}=150 \mathrm{GeV}$ & $e e$ & $e \mu$ & $\mu \mu$ & $\ell \ell$ \\
\hline \hline$H W W$ & $0.105 \pm 0.009$ & $0.225 \pm 0.019$ & $0.105 \pm 0.009$ & $0.436 \pm 0.037$ \\
$W W$ & $2.326 \pm 0.280$ & $4.752 \pm 0.572$ & $2.053 \pm 0.248$ & $9.132 \pm 1.100$ \\
$Z Z$ & $0.106 \pm 0.015$ & $0.164 \pm 0.023$ & $0.102 \pm 0.014$ & $0.372 \pm 0.053$ \\
$t \bar{t}$ & $0.009 \pm 0.002$ & $0.010 \pm 0.002$ & $0.017 \pm 0.003$ & $0.036 \pm 0.007$ \\
$W \gamma$ & $0.071 \pm 0.009$ & $0.127 \pm 0.016$ & $0.083 \pm 0.010$ & $0.281 \pm 0.035$ \\
$\mathrm{DY} \ell \ell$ & $0.624 \pm 0.160$ & $0.253 \pm 0.065$ & $0.323 \pm 0.083$ & $1.200 \pm 0.308$ \\
$\mathrm{QCD} / W+$ jet & $0.617 \pm 0.311$ & $0.563 \pm 0.283$ & $0.327 \pm 0.164$ & $1.507 \pm 0.759$ \\
\hline total background & $4.538 \pm 0.505$ & $6.562 \pm 0.697$ & $2.904 \pm 0.331$ & $14.005 \pm 1.508$ \\
\hline \hline data & 4 & 5 & 5 & 14 \\
\hline \hline
\end{tabular}

Table 9.7: Summary of the signal and background event yields for $m_{H}=150 \mathrm{GeV}$. 


\begin{tabular}{r||ccc|c}
\hline \hline$m_{H}=160 \mathrm{GeV}$ & $e e$ & $e \mu$ & $\mu \mu$ & $\ell \ell$ \\
\hline \hline$H W W$ & $0.138 \pm 0.011$ & $0.299 \pm 0.025$ & $0.141 \pm 0.012$ & $0.577 \pm 0.049$ \\
\hline$W W$ & $2.582 \pm 0.311$ & $5.017 \pm 0.604$ & $2.196 \pm 0.265$ & $9.794 \pm 1.179$ \\
$W Z$ & $0.105 \pm 0.015$ & $0.161 \pm 0.023$ & $0.099 \pm 0.014$ & $0.365 \pm 0.052$ \\
$Z Z$ & $0.012 \pm 0.002$ & $0.006 \pm 0.001$ & $0.018 \pm 0.003$ & $0.036 \pm 0.007$ \\
$t \bar{t}$ & $0.098 \pm 0.012$ & $0.163 \pm 0.020$ & $0.088 \pm 0.011$ & $0.349 \pm 0.043$ \\
$W \gamma$ & $0.691 \pm 0.065$ & $0.452 \pm 0.042$ & $0.000 \pm 0.000$ & $1.142 \pm 0.108$ \\
$D Y \ell \ell$ & $0.315 \pm 0.081$ & $0.182 \pm 0.047$ & $0.266 \pm 0.068$ & $0.763 \pm 0.196$ \\
QCD/W+jet & $0.616 \pm 0.310$ & $0.482 \pm 0.243$ & $0.237 \pm 0.119$ & $1.334 \pm 0.672$ \\
\hline total background & $4.418 \pm 0.495$ & $6.463 \pm 0.697$ & $2.903 \pm 0.318$ & $13.784 \pm 1.487$ \\
\hline \hline data & 5 & 6 & 5 & 16 \\
\hline \hline
\end{tabular}

Table 9.8: Summary of the signal and background event yields for $m_{H}=160 \mathrm{GeV}$.

\begin{tabular}{r||ccc|c}
\hline \hline$m_{H}=170 \mathrm{GeV}$ & $e e$ & $e \mu$ & $\mu \mu$ & $\ell \ell$ \\
\hline \hline$H W W$ & $0.130 \pm 0.011$ & $0.277 \pm 0.024$ & $0.133 \pm 0.011$ & $0.541 \pm 0.045$ \\
$W W$ & $2.708 \pm 0.326$ & $4.982 \pm 0.600$ & $2.277 \pm 0.274$ & $9.967 \pm 1.200$ \\
$Z Z$ & $0.105 \pm 0.015$ & $0.164 \pm 0.023$ & $0.110 \pm 0.015$ & $0.379 \pm 0.054$ \\
$t \bar{t}$ & $0.017 \pm 0.003$ & $0.008 \pm 0.001$ & $0.020 \pm 0.004$ & $0.044 \pm 0.008$ \\
$W \gamma$ & $0.106 \pm 0.014$ & $0.195 \pm 0.024$ & $0.098 \pm 0.012$ & $0.399 \pm 0.050$ \\
$\mathrm{DY} \ell \ell$ & $0.517 \pm 0.049$ & $0.452 \pm 0.042$ & $0.000 \pm 0.000$ & $0.969 \pm 0.091$ \\
$\mathrm{QCD} / W+$ jet & $0.572 \pm 0.288$ & $0.433 \pm 0.218$ & $0.237 \pm 0.119$ & $1.242 \pm 0.625$ \\
\hline total background & $4.339 \pm 0.486$ & $6.479 \pm 0.687$ & $3.008 \pm 0.328$ & $13.826 \pm 1.483$ \\
\hline \hline data & 5 & 7 & 6 & 18 \\
\hline \hline
\end{tabular}

Table 9.9: Summary of the signal and background event yields for $m_{H}=170 \mathrm{GeV}$.

\begin{tabular}{r||ccc|c}
\hline \hline$m_{H}=180 \mathrm{GeV}$ & $e e$ & $e \mu$ & $\mu \mu$ & $\ell \ell$ \\
\hline \hline$H W W$ & $0.100 \pm 0.008$ & $0.211 \pm 0.018$ & $0.098 \pm 0.008$ & $0.409 \pm 0.035$ \\
$W W$ & $2.677 \pm 0.322$ & $4.995 \pm 0.601$ & $2.220 \pm 0.267$ & $9.892 \pm 1.192$ \\
$Z Z$ & $0.119 \pm 0.017$ & $0.179 \pm 0.025$ & $0.136 \pm 0.020$ & $0.433 \pm 0.062$ \\
$t Z$ & $0.026 \pm 0.005$ & $0.008 \pm 0.001$ & $0.028 \pm 0.005$ & $0.062 \pm 0.013$ \\
$t \bar{t}$ & $0.124 \pm 0.016$ & $0.229 \pm 0.028$ & $0.107 \pm 0.014$ & $0.459 \pm 0.057$ \\
$W \gamma$ & $0.436 \pm 0.041$ & $0.380 \pm 0.036$ & $0.000 \pm 0.000$ & $0.816 \pm 0.077$ \\
$\mathrm{DY} \ell \ell$ & $0.315 \pm 0.081$ & $0.246 \pm 0.064$ & $0.266 \pm 0.068$ & $0.827 \pm 0.213$ \\
$\mathrm{QCD} / W+$ jet & $0.395 \pm 0.198$ & $0.263 \pm 0.133$ & $0.227 \pm 0.114$ & $0.884 \pm 0.445$ \\
\hline total background & $4.091 \pm 0.428$ & $6.299 \pm 0.660$ & $2.983 \pm 0.321$ & $13.373 \pm 1.396$ \\
\hline \hline data & 5 & 8 & 6 & 19 \\
\hline \hline
\end{tabular}

Table 9.10: Summary of the signal and background event yields for $m_{H}=180 \mathrm{GeV}$. 


\begin{tabular}{r||ccc|c}
\hline \hline$m_{H}=190 \mathrm{GeV}$ & $e e$ & $e \mu$ & $\mu \mu$ & $\ell \ell$ \\
\hline \hline$H W W$ & $0.065 \pm 0.006$ & $0.138 \pm 0.011$ & $0.064 \pm 0.005$ & $0.268 \pm 0.022$ \\
$W W$ & $2.639 \pm 0.318$ & $4.804 \pm 0.578$ & $2.180 \pm 0.263$ & $9.623 \pm 1.159$ \\
$W Z$ & $0.193 \pm 0.027$ & $0.176 \pm 0.025$ & $0.208 \pm 0.030$ & $0.577 \pm 0.082$ \\
$Z Z$ & $0.073 \pm 0.015$ & $0.008 \pm 0.002$ & $0.058 \pm 0.012$ & $0.139 \pm 0.027$ \\
$t \bar{t}$ & $0.144 \pm 0.018$ & $0.247 \pm 0.031$ & $0.127 \pm 0.016$ & $0.518 \pm 0.065$ \\
$W \gamma$ & $0.436 \pm 0.041$ & $0.218 \pm 0.021$ & $0.000 \pm 0.000$ & $0.654 \pm 0.062$ \\
$D Y \ell \ell$ & $0.386 \pm 0.100$ & $0.263 \pm 0.068$ & $0.193 \pm 0.049$ & $0.843 \pm 0.217$ \\
QCD $W+$ jet & $0.312 \pm 0.157$ & $0.261 \pm 0.131$ & $0.227 \pm 0.114$ & $0.800 \pm 0.403$ \\
\hline total background & $4.184 \pm 0.417$ & $5.977 \pm 0.634$ & $2.994 \pm 0.316$ & $13.154 \pm 1.356$ \\
\hline \hline data & 5 & 8 & 6 & 19 \\
\hline \hline
\end{tabular}

Table 9.11: Summary of the signal and background event yields for $m_{H}=190 \mathrm{GeV}$.

\begin{tabular}{r||ccc|c}
\hline \hline$m_{H}=200 \mathrm{GeV}$ & $e e$ & $e \mu$ & $\mu \mu$ & $\ell \ell$ \\
\hline \hline$H W W$ & $0.049 \pm 0.004$ & $0.102 \pm 0.008$ & $0.046 \pm 0.004$ & $0.197 \pm 0.017$ \\
$W W$ & $2.621 \pm 0.315$ & $4.484 \pm 0.540$ & $2.088 \pm 0.251$ & $9.193 \pm 1.107$ \\
$Z Z$ & $0.372 \pm 0.053$ & $0.161 \pm 0.023$ & $0.328 \pm 0.047$ & $0.860 \pm 0.123$ \\
$t \bar{t}$ & $0.167 \pm 0.033$ & $0.007 \pm 0.001$ & $0.128 \pm 0.025$ & $0.302 \pm 0.060$ \\
$W \gamma$ & $0.151 \pm 0.019$ & $0.275 \pm 0.034$ & $0.150 \pm 0.018$ & $0.576 \pm 0.072$ \\
$\mathrm{DY} \ell \ell$ & $0.305 \pm 0.078$ & $0.263 \pm 0.068$ & $0.390 \pm 0.100$ & $0.958 \pm 0.246$ \\
$\mathrm{QCD} / W+$ jet & $0.252 \pm 0.127$ & $0.253 \pm 0.128$ & $0.217 \pm 0.109$ & $0.722 \pm 0.364$ \\
\hline total background & $4.128 \pm 0.401$ & $5.662 \pm 0.596$ & $3.299 \pm 0.331$ & $13.089 \pm 1.318$ \\
\hline \hline data & 5 & 6 & 6 & 17 \\
\hline \hline
\end{tabular}

Table 9.12: Summary of the signal and background event yields for $m_{H}=200 \mathrm{GeV}$. 


\section{Chapter 10}

\section{Limit Extraction}

No excess in data was observed! So limits were set on the signal production crosssection $\sigma(p \bar{p} \rightarrow H) \times \mathrm{BR}\left(H \rightarrow W^{+} W^{-}\right)$using a binned likelihood method on the dilepton azimuthal separation angle distributions. The $\Delta \phi_{\ell \ell}$ distributions are shown in Figure 10.1. For reasons given in Chapter $7, \Delta \phi_{\ell \ell}$ was a good variable to discriminate the $H W W$ signal from the background since it would be small for the signal but not for the backgrounds.

With the presence of uncertainties in luminosity, signal acceptance and backgrounds, the cross-section limits were extracted in the framework of Bayesian statistics [59] under the premise of not losing the security of frequentist [63] coverage.

\subsection{Bayesian Statistics}

The Bayesian probability of a hypothesis is inferred by modifying the likelihood of null hypotheses with information about elemental factors in the hypothesis. Such inferences frequently use Bayes' theorem, which was derived first by the Reverend Tom Bayes. In practice, information about the hypothesis factors often mean a priori 

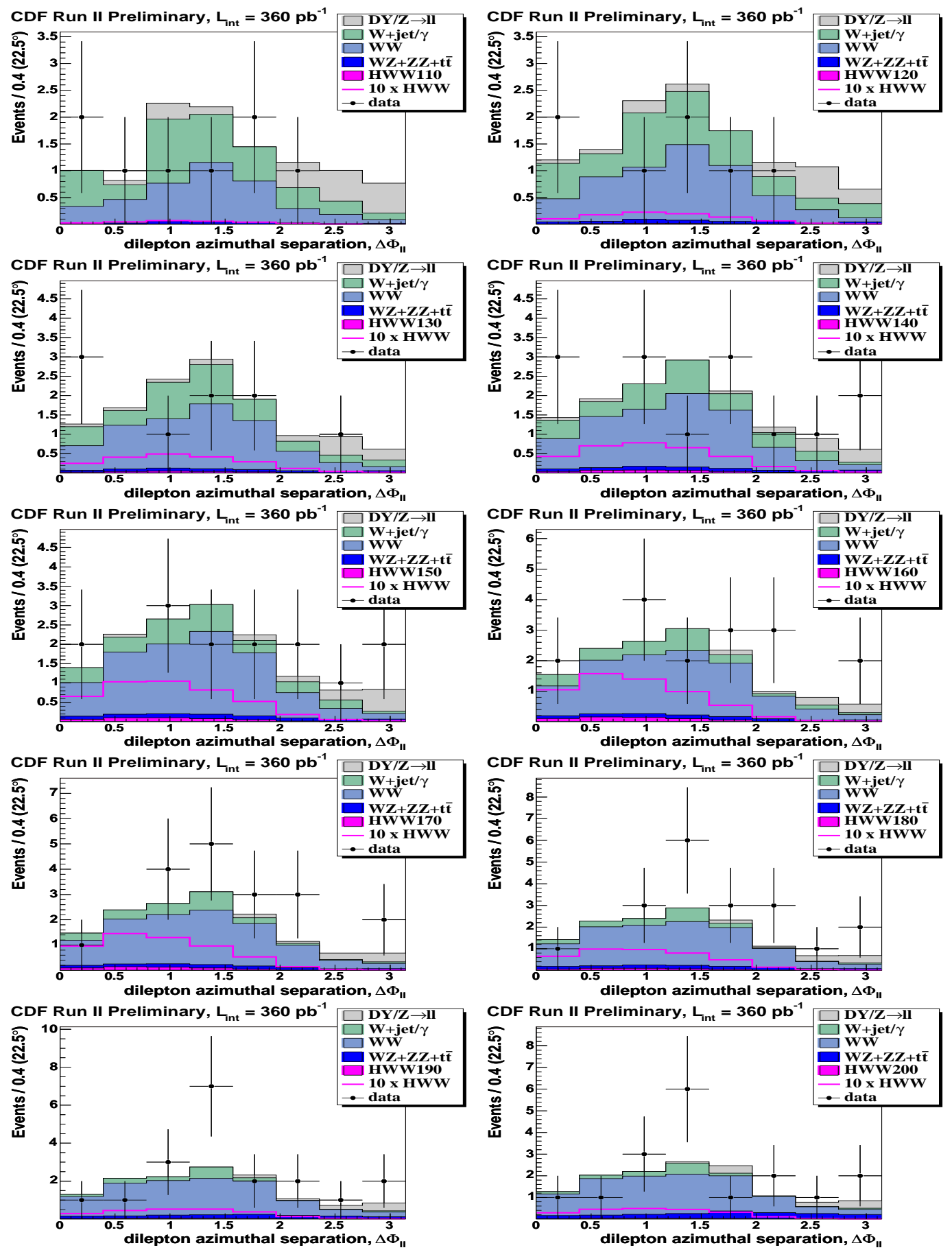

Figure 10.1: Dilepton azimuthal separation angle $\Delta \phi_{\ell \ell}$ distributions of the predicted signal and backgrounds and the observed data events for each of the analyzed Higgs masses $(\mathrm{GeV})$, as indicated to the right of $\mathbf{H W W}$ in the legends. 
probabilities, which are assigned to the hypothesis factors with arbitrary bias.

\subsubsection{Bayes' Theorem}

In the simplest case, where the hypothesis contains just one interesting variable $h$ that is related to just one observable variable $n$, the conditional and marginal probabilities of $h$ and $n$ in the Bayes' theorem are related by

$$
\operatorname{Pr}(h \mid n)=\frac{\operatorname{Pr}(n \mid h) \operatorname{Pr}(h)}{\int \operatorname{Pr}(n \mid h) \operatorname{Pr}(h) d h}
$$

where

$\operatorname{Pr}(h)$ is the prior and marginal probability of $h$, which is inferred prior to the availability of $n$;

$\operatorname{Pr}(n \mid h)$ is the conditional probability of $n$ for given $h$, i.e. the probability of observing $n$ if $h$ is true;

$\operatorname{Pr}(n)=\int \operatorname{Pr}(n \mid h) \operatorname{Pr}(h) d h$ is the prior and marginal probability of $n$, serving as a normalization factor in Equation 10.1;

$\operatorname{Pr}(h \mid n)$ is the posterior and conditional probability of $h$ for given $n$.

In the slightly more complicated case of $H W W$ production cross-section limit extraction, where the hypothesis contains one interesting variable (signal cross-section $\sigma$ ) and several nuisance parameters (signal acceptance $\epsilon$, integrated luminosity $\mathcal{L}$ and backgrounds $b$ ) that are related to an observable variable (number of events in data $n)$, the probabilities of or among these variables in the Bayes' theorem are related by

$$
\operatorname{Pr}(\sigma \mid n, \epsilon, \mathcal{L}, b)=\frac{\operatorname{Pr}(n \mid \sigma, \epsilon, \mathcal{L}, b) \operatorname{Pr}(\sigma) \operatorname{Pr}(\epsilon) \operatorname{Pr}(\mathcal{L}) \operatorname{Pr}(b)}{\iiint \int \operatorname{Pr}(n \mid \sigma, \epsilon, \mathcal{L}, b) \operatorname{Pr}(\sigma) \operatorname{Pr}(\epsilon) \operatorname{Pr}(\mathcal{L}) \operatorname{Pr}(b) d b d \mathcal{L} d \epsilon d \sigma}
$$


The question is how to transform all of these probabilities into functions that can be used to calculate $95 \%$ Bayesian credibility level (CL) ${ }^{1}$ limits.

\subsubsection{Likelihood of Null Hypothesis}

The probability density function (pdf) of observing $n$ events for a given set of signal cross-section $\sigma$, signal acceptance $\epsilon$, integrated luminosity $\mathcal{L}$ and background $b$ is best described by Poisson [64]

$$
\operatorname{Pr}(n \mid \sigma, \epsilon, \mathcal{L}, b)=\frac{(\sigma \epsilon \mathcal{L}+b)^{n}}{n !} e^{-(\sigma \epsilon \mathcal{L}+b)}
$$

for a situation of low statistics, such as this $H W W$ dilepton search.

\subsubsection{Priors}

The prior probability density functions, $\operatorname{Pr}(\sigma)$ for signal cross-section, $\operatorname{Pr}(\epsilon)$ for signal acceptance, $\operatorname{Pr}(\mathcal{L})$ for integrated luminosity and $\operatorname{Pr}(b)$ for background, were assigned on the idea that priors together with the likelihood function of null hypotheses must yield a proper posterior. Priors were assigned probability density functions according to the nature of the uncertainty in each variable.

\subsubsection{Signal Cross-Section}

Nothing is known about the true value of the $H W W$ cross-section; no information is available for setting the prior pdf of the signal cross-section. This lack of information reveals a weakness of the Bayesian approach: there is no universally accepted method to obtain the prior pdf of a variable in this situation. However, previous studies e.g. [62] have proposed several solutions, a couple of them being in

\footnotetext{
${ }^{1}$ An equivalent term to the frequentist confidence level although not always equaling the value.
} 
the form of $\operatorname{Pr}(\sigma)=\sigma^{-\iota}$, where

$\iota=0$ corresponds to a uniform pdf and yields an upper limit consistent with the frequentist approach to a Poisson process;

$\iota=1$ corresponds to a log-uniform pdf and yields a lower limit consistent with the frequentist approach to a Poisson process.

Since the upper limits on the cross-section of a Poisson process were sought, the $H W W$ signal cross-section prior pdf was chosen to be uniform,

$$
\operatorname{Pr}(\sigma)=\text { const for } 0<\sigma<\sigma_{\max } \text { and } \int_{0}^{\sigma_{\max }} \operatorname{Pr}(\sigma) d \sigma=1
$$

where $\sigma_{\max } \rightarrow \infty$ in the case of nothing at all about $\sigma$ was known.

\subsubsection{Signal Acceptance}

Our knowledge of signal acceptance $\epsilon$ can be summarized as a central value $\epsilon_{0}$ with uncertainty $\delta_{\epsilon}$, as estimated in Chapter 8 . With the knowledge in such a form as $\epsilon=\epsilon_{0} \pm \delta_{\epsilon}$ it might be tempting to think of a Gaussian $\epsilon$ prior pdf,

$$
\operatorname{Pr}(\epsilon)=\frac{1}{\delta_{\epsilon} \sqrt{2 \pi}} \exp \left[-\frac{1}{2}\left(\frac{\epsilon-\epsilon_{0}}{\delta_{\epsilon}}\right)^{2}\right]
$$

However, this kind of prior pdf together with the uniform signal cross-section prior pdf and any background prior(s) would yield an improper posterior in Equation (10.2) to diverge the likelihood integral over signal cross-section phase space in Equation (10.15). Previous studies e.g. [60, 61] have proposed a log-normal pdf for the prior of signal acceptance instead. 
Suppose the pdf of observing $m$ events in the subsidiary measurement of a rather small signal acceptance $\epsilon$ is described by Poisson

$$
\operatorname{Pr}(m \mid \epsilon)=\frac{(\kappa \epsilon)^{m}}{m !} e^{-\kappa \epsilon}
$$

where $\epsilon_{0}=(m+1) / \kappa, \delta_{\epsilon}^{2}=(m+1) / \kappa^{2}$ and the scaling constant $\kappa=\epsilon_{0} / \delta_{\epsilon}^{2}$. Exercising Equation 10.1 and assigning a uniform prior pdf to $\epsilon$, the posterior pdf of $\epsilon$ for a given $m$,

$$
\begin{aligned}
\operatorname{Pr}(\epsilon \mid m) & =\frac{\operatorname{Pr}(m \mid \epsilon) \operatorname{Pr}(\epsilon)}{\int \operatorname{Pr}(m \mid \epsilon) \operatorname{Pr}(\epsilon) d \epsilon} \\
& \propto \frac{(\kappa \epsilon)^{m}}{m !} e^{-\kappa \epsilon}
\end{aligned}
$$

is the prior probability density function of signal acceptance $\epsilon$ in Equation 10.2. Note that the $\operatorname{Pr}(\epsilon)$ in Equation 10.5 is for the measured $\epsilon$ while the $\operatorname{Pr}(\epsilon)$ in Equation 10.8 is for the true value of $\epsilon$.

In the case of this $H W W$ dilepton search, $m$ was large, $\delta_{\epsilon} / \epsilon_{0}$ was small and $\left.\operatorname{Pr}(\epsilon \mid m)\right|_{m \rightarrow \infty}$ approximated a narrow Gaussian.

\subsubsection{Integrated Luminosity}

Our knowledge of integrated luminosity can be summarized as a central value $\mathcal{L}_{0}$ with uncertainty $\delta_{\mathcal{L}}$, as measured by CLC.

The prior probability density function of integrated luminosity is

$$
\begin{aligned}
\left.\operatorname{Pr}(\mathcal{L} \mid m)\right|_{m \rightarrow \infty} & \propto \frac{(\kappa \mathcal{L})^{m}}{m !} e^{-\kappa \mathcal{L}} \\
& \approx \frac{1}{\delta_{\mathcal{L}} \sqrt{2 \pi}} \exp \left[-\frac{1}{2}\left(\frac{\mathcal{L}-\mathcal{L}_{0}}{\delta_{\mathcal{L}}}\right)^{2}\right]
\end{aligned}
$$

where the approximation holds for a large number of observed events $m$ in the subsidiary luminosity measurement. 
With the resemblance in knowledge form, the prior pdf assignment to luminosity was the same as the prior pdf assignment to signal acceptance.

\subsubsection{Backgrounds}

Our knowledge of backgrounds can each be summarized as a central value $b_{0}$ with uncertainty $\delta_{b}$, as estimated in Chapter 9 . Due to $\Delta \phi_{\ell \ell}$ shape difference, backgrounds were grouped into $W W$ and the sum of the rest.

The prior probability density function of each background group is

$$
\begin{aligned}
\left.\operatorname{Pr}(b \mid m)\right|_{m \rightarrow \infty} & \propto \frac{(\kappa b)^{m}}{m !} e^{-\kappa b} \\
& \approx \frac{1}{\delta_{b} \sqrt{2 \pi}} \exp \left[-\frac{1}{2}\left(\frac{b-b_{0}}{\delta_{b}}\right)^{2}\right]
\end{aligned}
$$

where the approximation holds for a large number of observed events $m$ in the subsidiary measurement of background expectation ${ }^{2}$.

With the resemblance in knowledge form, the prior pdf assignment to each background group was the same as the prior pdf assignment to signal acceptance.

\subsubsection{Posterior}

At this stage, the posterior probability density function of the $H W W$ signal cross-section $\operatorname{Pr}(\sigma \mid n, \epsilon, \mathcal{L}, b)$ for observed number of events in data $n$, estimated signal acceptance $\epsilon$, integrated luminosity $\mathcal{L}$ and expected backgrounds $b$, defined as in Equation 10.2, has the likelihood of null hypotheses and all the priors properly assigned

\footnotetext{
${ }^{2}$ Background prior propriety is considered a secondary issue by most of the Bayesians because there is always enough information in data to make a proper posterior even when both signal $\sigma \epsilon$ and background $b$ priors are improper.
} 
probability density functions for practical posterior calculation.

\subsection{Binned Likelihood}

An $N$-binned likelihood in the Bayesian statistics is the product of $N$ one-binned posteriors with the same bin size over the same variable range.

$$
\begin{aligned}
\operatorname{Pr}(\sigma \mid n, \epsilon, \mathcal{L}, b) & =\prod_{j=1}^{N} \operatorname{Pr}\left(\sigma \mid n_{j}, \epsilon_{j}, \mathcal{L}, b_{j}\right) \\
\ln [\operatorname{Pr}(\sigma \mid n, \epsilon, \mathcal{L}, b)] & =\sum_{j=1}^{N} \ln \left[\operatorname{Pr}\left(\sigma \mid n_{j}, \epsilon_{j}, \mathcal{L}, b_{j}\right)\right]
\end{aligned}
$$

where the posterior of the $j$ th bin is obtained with the likelihood of null hypotheses and the priors of the $j$ th bin.

\section{$10.3 \quad 95 \%$ CL Upper Limit}

Based on the soundness of likelihood estimate, there should be greater than 95\% normalized probability for the interesting variable to have its true value in the quoted $95 \%$ CL interval on its value. There should be greater than $95 \%$ normalized probability for the $H W W$ cross-section to have its true value below the quoted $95 \%$ CL upper limit on it for each Higgs mass.

Integration of the binned likelihood over cross-section phase space was carried out for setting $95 \% \mathrm{CL}$ upper limits on the $H W W$ signal cross-section,

$$
\frac{\int_{0}^{\sigma_{\lim }} \operatorname{Pr}(\sigma \mid n, \epsilon, \mathcal{L}, b) d \sigma}{\int_{0}^{\sigma_{\max }} \operatorname{Pr}(\sigma \mid n, \epsilon, \mathcal{L}, b) d \sigma} \geq 95 \%
$$

where $\sigma_{\text {lim }}$ stands for the $95 \%$ CL upper limit on cross-section. Note that the constant prior normalization factors are canceled due to their appearances in both numerator and denominator of Equation 10.15; only shapes matter. 


\subsection{Numerical Computation}

In implementing the binned likelihood integral numerically,

signal cross-section:

prior $\operatorname{Pr}(\sigma)=0.02 \mathrm{pb}^{-1}$ for $0<\sigma<50.0 \mathrm{pb}^{3}$;

prior $\operatorname{Pr}(\sigma)=0$ otherwise;

integration step size $d \sigma=0.05 \mathrm{pb}$.

signal acceptance:

the log-normal prior $\operatorname{Pr}(\epsilon)$ was approximated by a normal one since the uncertainty was relatively small compared to the central value of signal acceptance;

the signal acceptance was smeared by a Gaussian random number generating $\mathrm{C}++$ function with the central value of the signal acceptance and the uncertainty in it as the mean and the standard deviation;

the smeared signal acceptance was distributed into $\Delta \phi_{\ell \ell}$ bins according to the MC prediction about signal $\Delta \phi_{\ell \ell}$ distribution.

integrated luminosity:

the log-normal prior $\operatorname{Pr}(\mathcal{L})$ was approximated by a normal one since the uncertainty was relatively small compared to the central value of integrated

\footnotetext{
${ }^{3}$ It approximated the condition of infinity well enough to set the upper edge of the uniform signal cross-section prior $\mathrm{pdf}$ at $\sigma_{\max }=50.0 \mathrm{pb}$ since the upper limits on the signal production cross-section would not change if the $\sigma_{\max }$ value was even higher.
} 
luminosity;

the integrated luminosity was smeared by a Gaussian random number generating $\mathrm{C}++$ function with the central value of the integrated luminosity and the uncertainty in it as the mean and the standard deviation.

backgrounds:

the log-normal prior $\operatorname{Pr}(b)$ was approximated by a normal one since the uncertainty was relatively small compared to the central value of each background group;

each background group was smeared by a Gaussian random number generating $\mathrm{C}++$ function with the central value of the background and the uncertainty in it as the mean and the standard deviation;

each smeared background group was distributed into $\Delta \phi_{\ell \ell}$ bins according to the MC prediction about each background group's $\Delta \phi_{\ell \ell}$ distribution.

In each integration step $s$, where the hypothesis was $0.05(s-1)<\sigma<0.05 s$ pb, smearing of prior variables, looping over all bins and calculating likelihood were performed a thousand times and the average likelihood out of a thousand was taken as the likelihood of this step's hypothesis.

After running through all the integration steps and calculating all the step likelihoods, the fraction of the likelihood area between 0 and each step to the likelihood area between 0 and $50.0 \mathrm{pb}$ was computed. The observed likelihood distributions are shown in Figure 10.2. The cross-section upper bound of which step the fraction 
became greater than or equal to $95 \%$,

$$
\frac{\sum_{s=1}^{s_{\lim }} \operatorname{Pr}(\sigma=0.05 s \mid n, \epsilon, \mathcal{L}, b)}{\sum_{s=1}^{1000} \operatorname{Pr}(\sigma=0.05 s \mid n, \epsilon, \mathcal{L}, b)} \geq 95 \%
$$

as a numeric form of Equation 10.15, was taken as the 95\% CL cross-section upper limit. This numerical procedure was followed to compute an observed 95\% CL upper limit on the $H W W$ cross-section for each Higgs mass.

\subsection{Pseudoexperiments}

No matter what is observed in data, expected limits are calculated assuming perfect agreement between data and background prediction. Calculating the expected limits is the same as calculating the observed limits, including the level of confidence or credibility, except for the number of events observed in data. For the expected limits, the number of events observed in data $n$ was simulated in the following way:

1. background was smeared by a Gaussian random number generating $\mathrm{C}++$ function with the central value of the background and the uncertainty in it as the mean and the standard deviation;

2. smeared background was fluctuated by a Poisson random number generating $\mathrm{C}++$ function with the Gaussian smeared background as mean;

3. fluctuated smeared background was randomly assigned a $\Delta \phi_{\ell \ell}$ bin according to the expected background $\Delta \phi_{\ell \ell}$ distribution by the $\mathrm{C}++$ function in ROOT [65] Double_t TH1: :GetRandom();

4. under the premise of perfect agreement between data and the expected total background, the randomized $\Delta \phi_{\ell \ell}$ distribution of the fluctuated smeared back- 


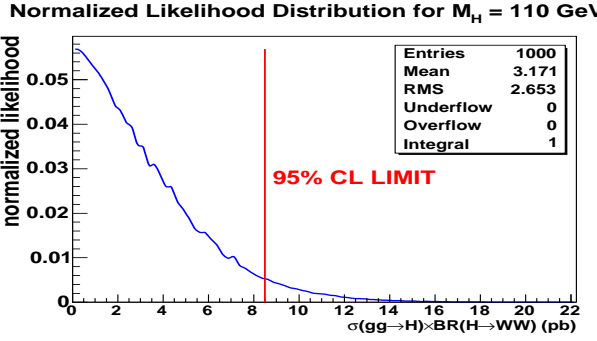

Normalized Likelihood Distribution for $M_{\mathbf{H}}=130 \mathrm{GeV}$

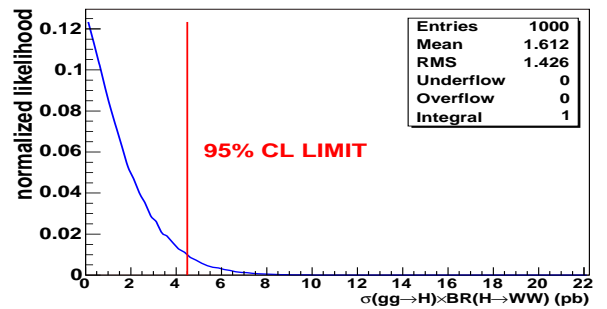

Normalized Likelihood Distribution for $M_{H}=150 \mathrm{GeV}$

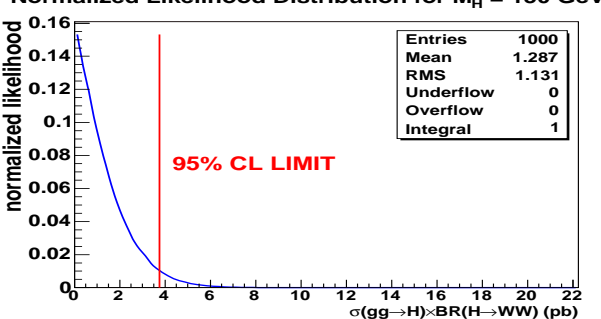

Normalized Likelihood Distribution for $M_{H}=170 \mathrm{GeV}$

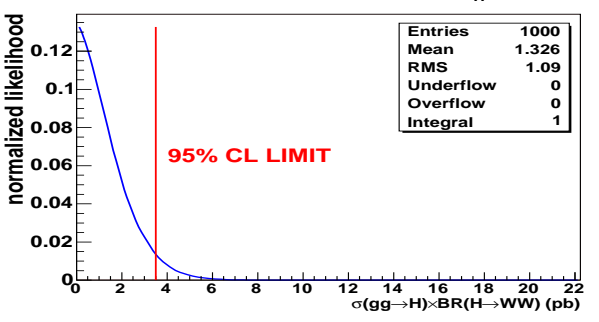

Normalized Likelihood Distribution for $M_{H}=190 \mathrm{GeV}$

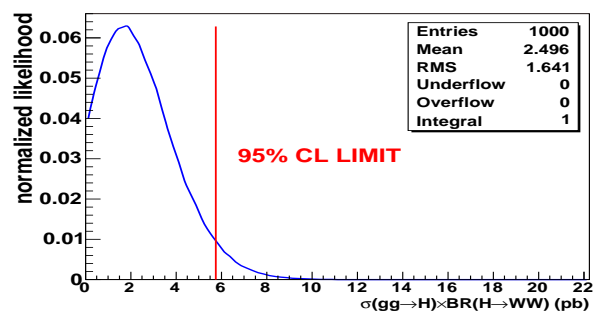

Normalized Likelihood Distribution for $M_{H}=120 \mathrm{GeV}$

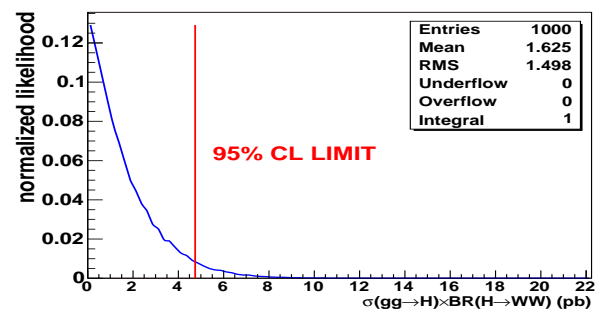

Normalized Likelihood Distribution for $M_{H}=140 \mathrm{GeV}$

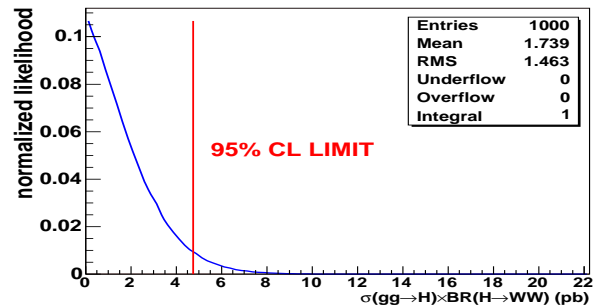

Normalized Likelihood Distribution for $M_{H}=160 \mathrm{GeV}$
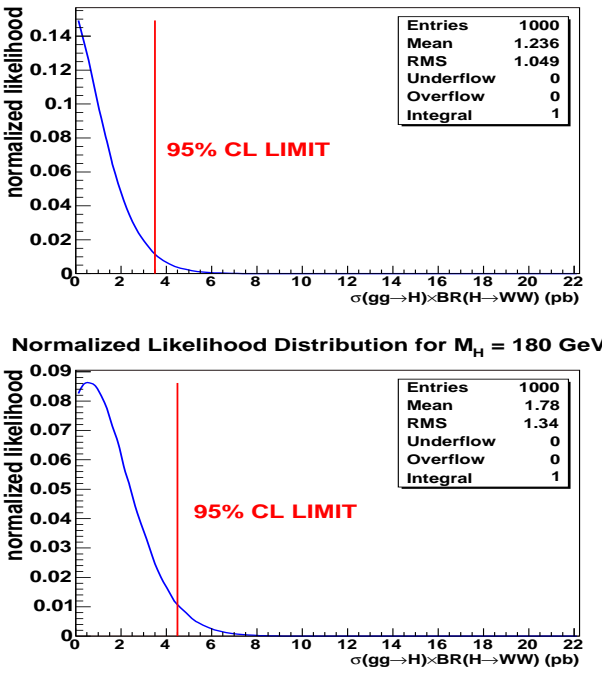

Normalized Likelihood Distribution for $\mathbf{M}_{\mathbf{H}}=200 \mathrm{GeV}$

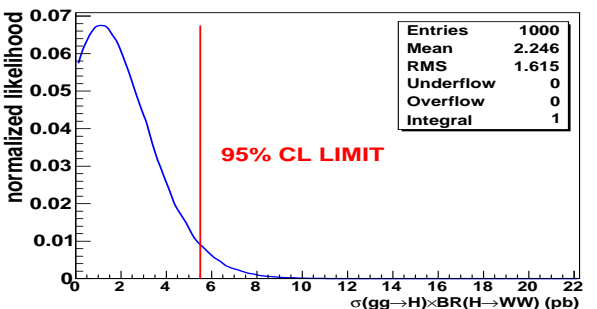

Figure 10.2: Observed $H W W$ cross-section likelihood distributions for each of the analyzed Higgs masses. 


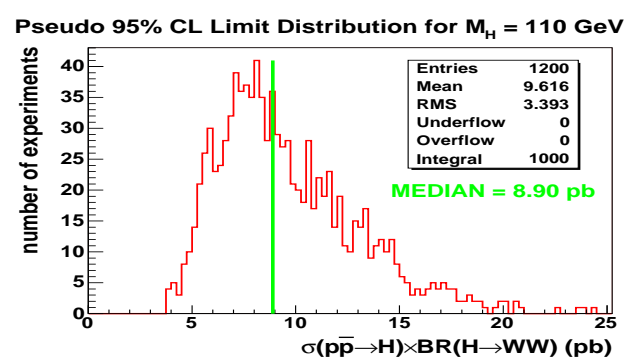

Pseudo 95\% CL Limit Distribution for $M_{H}=130 \mathrm{GeV}$

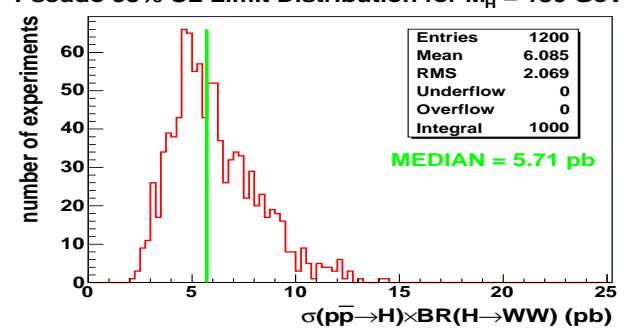

Pseudo 95\% CL Limit Distribution for $M_{H}=150 \mathrm{GeV}$

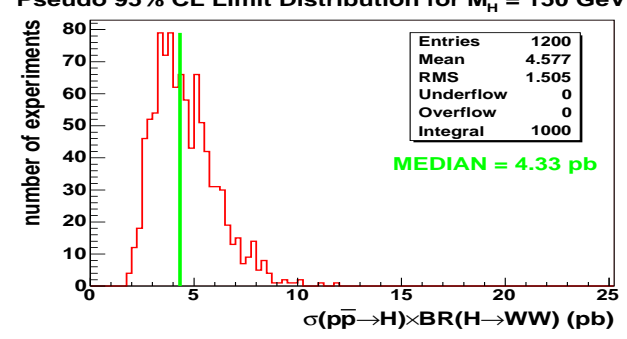

Pseudo 95\% CL Limit Distribution for $M_{H}=170 \mathrm{GeV}$
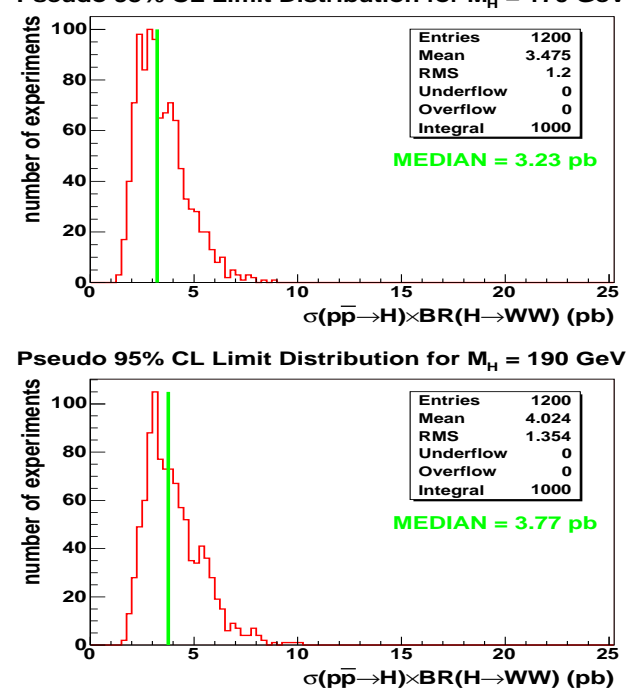

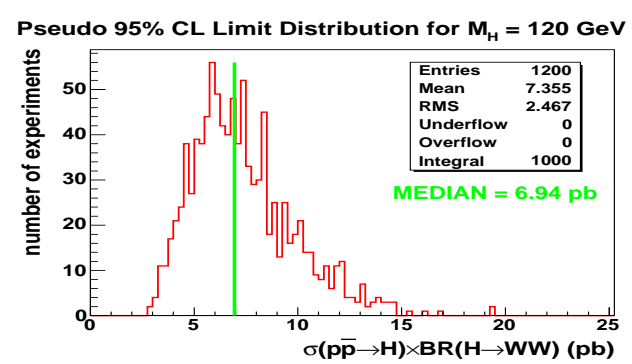

Pseudo 95\% CL Limit Distribution for $M_{H}=140 \mathrm{GeV}$

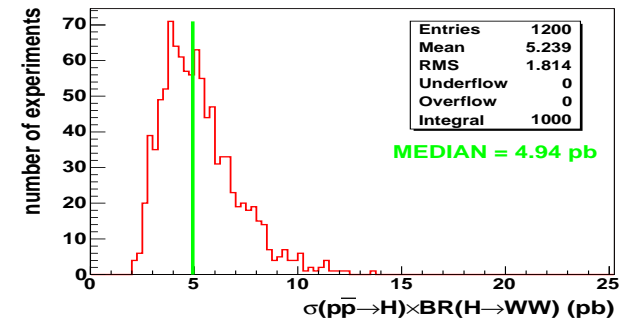

Pseudo 95\% CL Limit Distribution for $M_{H}=160 \mathrm{GeV}$

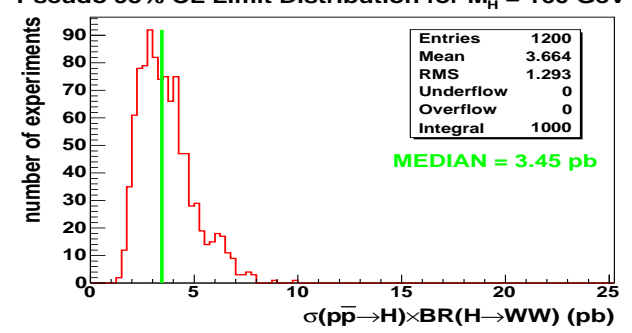

Pseudo 95\% CL Limit Distribution for $M_{H}=180 \mathrm{GeV}$

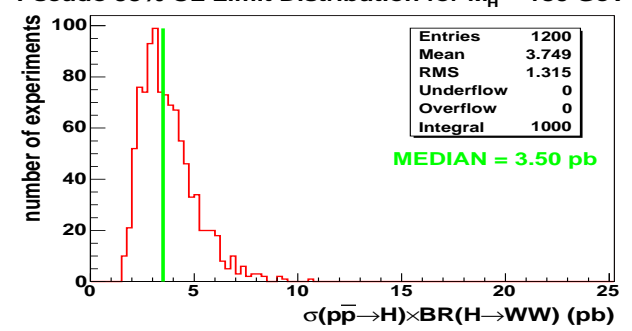

Pseudo 95\% CL Limit Distribution for $M_{H}=200 \mathrm{GeV}$

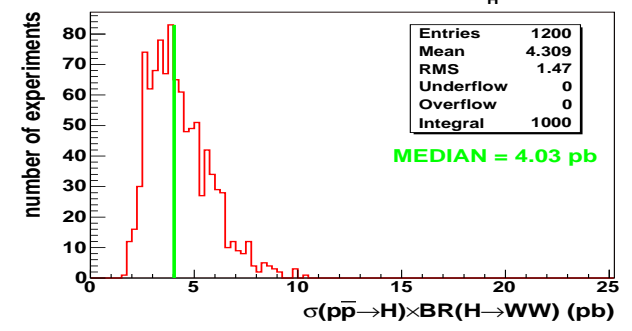

Figure 10.3: Expected $H W W$ cross-section upper limit distributions for each of the analyzed Higgs masses. 
ground was the $\Delta \phi_{\ell \ell}$ distribution of simulated data for the expected limit calculation.

In pseudoexperiments, the numeric procedure with the extra bit of data simulation was repeated one thousand times and the median 95\% CL cross-section upper limit out of a thousand was taken as the expected 95\% CL cross-section upper limit.

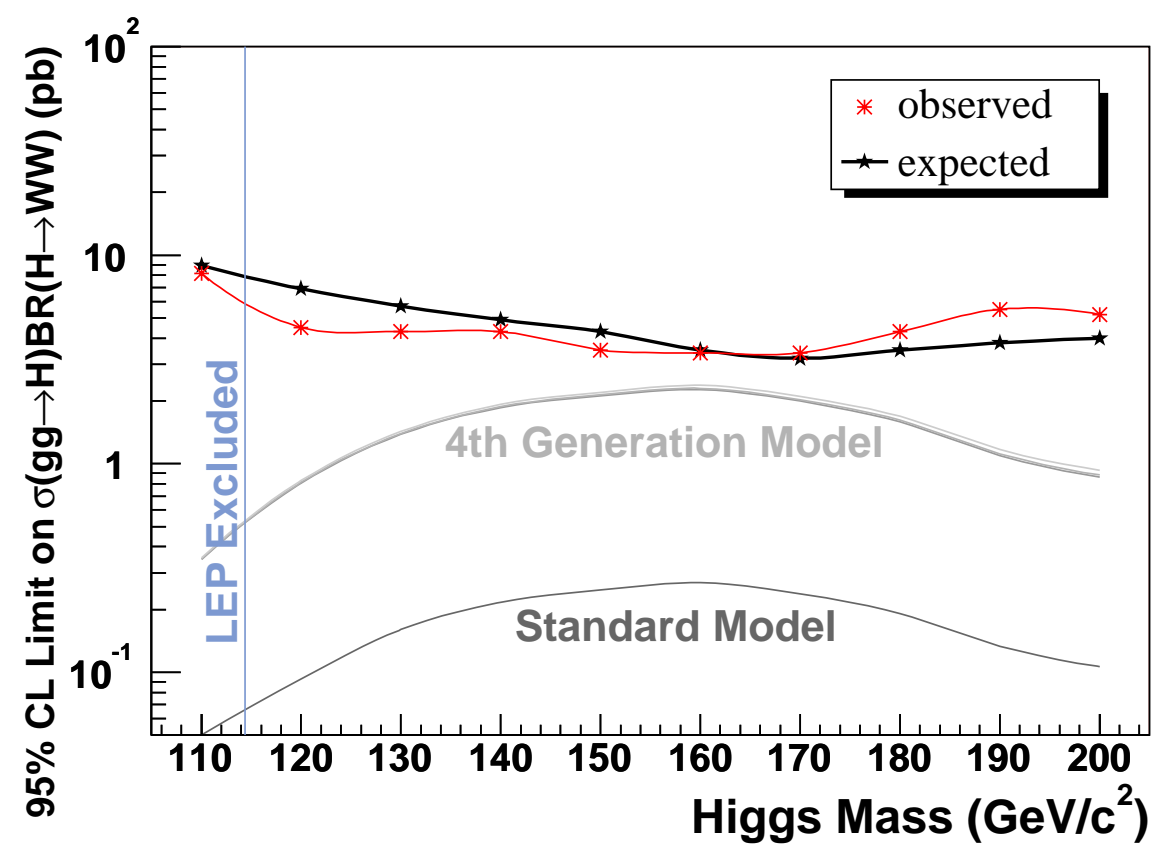

Figure 10.4: Summary of the expected and observed $\sigma(p \bar{p} \rightarrow H) \times \mathrm{BR}\left(H \rightarrow W^{+} W^{-}\right)$ upper limits at 95\% CL as a function of the Higgs mass.

\begin{tabular}{ccccccccccc}
\hline \hline$m_{H}\left(\mathrm{GeV} / c^{2}\right)$ & 110 & 120 & 130 & 140 & 150 & 160 & 170 & 180 & 190 & 200 \\
\hline \hline expected $(\mathrm{pb})$ & 8.9 & 6.9 & 5.7 & 4.9 & 4.3 & 3.4 & 3.2 & 3.5 & 3.8 & 4.0 \\
\hline observed $(\mathrm{pb})$ & 8.3 & 4.5 & 4.4 & 4.6 & 3.5 & 3.2 & 3.4 & 4.3 & 5.5 & 5.2 \\
\hline \hline
\end{tabular}

Table 10.1: Summary of the expected and observed $\sigma(p \bar{p} \rightarrow H) \times \mathrm{BR}\left(H \rightarrow W^{+} W^{-}\right)$ upper limits at $95 \%$ CL as a function of the Higgs mass. 
This repetition was performed to compute for an expected 95\% CL upper limit on the $H W W$ cross-section for each Higgs mass. The pseudoexperimental 95\% CL upper limit distributions, one for each Higgs mass, are shown in Figure 10.3.

\subsection{5\% CL Upper Limits on the $H W W$ Signal Cross-Section}

Both the observed and expected 95\% CL $H W W$ cross-section upper limits as a function of the Higgs mass are summarized in Figure 10.4 and Table 10.1. 


\section{Chapter 11}

\section{Conclusion}

The first search at CDF for the SM Higgs boson in the $H \rightarrow W^{+} W^{-}$decay channel was performed in $p \bar{p}$ collisions at $\sqrt{s}=1.96 \mathrm{TeV}$. The amount of analyzed data corresponds to an integrated luminosity of $\mathcal{L}_{\text {int }}=360 \pm 22 \mathrm{pb}^{-1}$. A signal in the Higgs mass range of $110 \leq m_{H} \leq 200 \mathrm{GeV} / c^{2}$ was searched for in the $W^{+} W^{-} \rightarrow \ell^{+} \nu_{\ell} \ell^{-} \bar{\nu}_{\ell}$ decay channels where each lepton $\ell$ can be an electron $e$, a muon $\mu$ or a tau $\tau$ that further decays to an electron or a muon. Events characterized by two leptons with a small dilepton invariant mass together with large missing transverse energy were sought. Good agreement between data and the SM background expectation was observed in all the $e e, e \mu$ and $\mu \mu$ channels for each of the analyzed Higgs masses. Observing no signal-like excess in data, upper limits on the signal production cross-section were set at $95 \%$ Bayesian credibility level for the analyzed Higgs mass range, as shown in Figure and Table 10.1. Results of this analysis have been published in Physical Review Letters 97081802 (2006) [66]. 


\section{Bibliography}

[1] S. L. Glashow, Partial Symmetries of Weak Interactions, Nuclear Physics 22579 (1961);

S. Weinberg, A Model of Leptons, Physical Review Letters 191264 (1967).

[2] D. H. Perkins, Introduction to High Energy Physics (1987).

[3] F. Halzen and A. D. Martin, Quarks and Leptons (1984).

[4] Louis de Broglie, Recherches sur la Théorie des Quanta (thesis for a doctoral degree at the University Paris Sorbonne, 1924).

[5] E. Schrödinger, Quantisierung als Eigenwertproblem, Annalen der Physik (Leipzig) 79 361, 489, 734 (1926), 80437 (1926), 81109 (1926).

[6] O. Klein, Zeitschrift für Physik 37895 (1926);

V. Fock, Zeitschrift für Physik 38242 (1926);

W. Gordon, Zeitschrift für Physik 40117 (1926).

[7] P. A. M. Dirac, Quantum Theory of the Electron, Proceedings of the Royal Society A117 610 (1928), A118 351 (1928). 
[8] P. A. M. Dirac, Principles of Quantum Mechanics (1st edition in 1930);

Proceedings of the Royal Society A126 360 (1930).

[9] Particle Data Group, Review of Particle Physics (2006).

[10] N. Cabibbo, Unitary Symmetry and Leptonic Decays, Physical Review Letters 10531 (1963).

[11] E. Galois, Cuvres Mathématiques, Journal de Mathématiques pures et Appliquées XI 385 (1846).

[12] W. Pauli, Zur Quantenmechanik des Magnetischen Elektrons, Zeitschrift für Physik 43601 (1927).

[13] W. Voigt, Nachrichten von der Königlicher Gesellschaft den Wissenschaft zu Göttingen 241 (1887);

J. Larmor, Philosophical Transactions of the Royal Society 190205 (1897);

H. A. Lorentz, Verhandelingen der Koninklijke Nederlandse Akademie van Wetenschappen I 427 (1899), IV 669 (1904);

H. Poincaré, Comptes Rendues 1401504 (1905).

[14] V. Fock, Zeitschrift für Physik 39226 (1926);

F. London, Zeitschrift für Physik 42375 (1927);

H. Weyl, Zeitschrift für Physik 56330 (1929).

[15] J. Goldstone, A. Salam and S. Weinberg, Broken Symmetries, Physical Review 127965 (1962); 
S. L. Glashow, J. Iliopoulos and L. Maiani, Weak Interactions with LeptonHadron Symmetry, Physical Review D 21285 (1970).

[16] B. R. Martin and G. Shaw, Particle Physics (1997).

[17] S. Weinberg, Mixing Angle in Renormalizable Theories of Weak and Electromagnetic Interactions, Physical Review D 51962 (1972).

[18] ALEPH Collaboration, Precise Determination of the Number of Families with Light Neutrinos and of the $Z$ Boson Partial Widths, Physics Letter B 235399 (1990).

[19] P. W. Higgs, Broken Symmetries, Massless Particles and Gauge Fields, Physics Letter 12132 (1964).

[20] H. E. Haber, G. Kane, S. Dawson and J. F. Gunion, the Higgs Hunter's Guide (1989, errata in hep-ph/9302272).

[21] R. Davis et al., Measurement of the Solar Electron Neutrino Flux with the Homestake Chlorine Detector, Astrophysical Journal 496505 (1998);

Super-Kamiokande Collaboration, Evidence for Oscillation of Atmospheric Neutrinos, Physical Review Letters 811562 (1998).

[22] H. Georgi and S. L. Glashow, Unity of All Elementary Particle Forces, Physical Review Letters 32438 (1974);

J. C. Pati and A. Salam, Lepton Number as the 4th Color, Physical Review D 10275 (1974). 
[23] J. Wess and B. Zumino, Supergauge Transformations in Four-Dimensions, Nuclear Physics B 7039 (1974);

S. Dimopoulos and H. Georgi, Softly Broken Supersymmetry and SU(5), Nuclear Physics B 193150 (1981).

[24] E. Arik, O. Cakir, S. A. Cetin and S. Sultansoy, Consequences of the Extra SM Families on the Higgs Boson Production at Tevatron and LHC, Physical Review D 66033003 (2002).

[25] M. Spira, HIGLU: A Program for the Calculation of the Total Higgs Production Cross-Section at Hadron Colliders via Gluon Fusion Including QCD Corrections (1995).

[26] D. Bardin et al., ZFITTER V6.21: A Semianalytical Program for Fermion Pair Production in $e^{+} e^{-}$Annihilation, Computer Physics Communications 133229 (2001).

[27] LEP Working Group for the Higgs boson Searches, Search for the Standard Model Higgs Boson at LEP, Physics Letter B 56561 (2003).

[28] Higgs Working Group Collaboration, Report of the Tevatron Higgs Working Group, hep-ph/0010338 (2000).

[29] CDF Collaboration, Search for Higgs Bosons Decaying into $b \bar{b}$ and Produced in Association with a Vector Boson in $p \bar{p}$ Collisions at $1.8 \mathrm{TeV}$, Physical Review Letters 95051801 (2005). 
[30] DØ Collaboration, Search for the Higgs Boson in $H \rightarrow W W^{(*)}$ Decays in $p \bar{p}$ Collisions at $\sqrt{s}=1.96 \mathrm{TeV}$, Physical Review Letters 96011801 (2006).

[31] CDF Collaboration, the CDF II Detector Technical Design Report (1996).

[32] Y. Ishizawa and J. Nielsen, Trigger Efficiencies for High $E_{T}$ Electrons, CDF internal note 7401 (2004).

[33] E. Halkiadakis and B. Han, MET_PEM Trigger Efficiency for Pheonix Electrons, CDF internal note 7940 (2005).

[34] V. Martin, High $P_{T}$ Muon ID Cuts and Efficiencies for Use with 5.3.1 Data and 5.3.3 MC, CDF internal note 7367 (2004).

[35] CDF Collaboration, Luminosity Monitoring and Measurement at CDF, Nuclear Instruments and Methods A 44337 (2000).

[36] R. G. Wagner, Electron Identification for Run II: Algorithms, CDF internal note $5456(2000)$.

[37] C. Hill, J. Incandela and C. Mill, Electron Identification in Offline Release 5.3, CDF internal note 7309 (2004).

[38] M. Griffiths, B. Heinemann and G. Manca, Central Electron Identification Efficiencies at Medium Energy, CDF internal note 7233 (2004).

[39] T. Nelson, R. Snider and D. Stuart, Forward Electron Tracking with the PhoenixMods Package, CDF internal note 6278 (2003). 
[40] A. Taffard, CDF Run II Cosmic Ray Tagger and its Performance, CDF internal note 6100 and 6255 (2003).

[41] O. Gonzalez et al., Identification Efficiency for Intermediate $P_{T}$ Muons, CDF internal note 7197 (2004).

[42] CDF Collaboration, Determination of the Jet Energy Scale at the Collider Detector at Fermilab, Nuclear Instruments and Methods A 566375 (2006).

[43] S. Chuang, M. Coca and M. Kruse, Lepton Fake Estimate in 314.2 pb $^{-1}$ CDF Run II Data, CDF internal note 7497 (2005).

[44] S. Chuang, M. Coca and M. Kruse, Search for the SM Higgs Bosons via $g g \rightarrow$ $H \rightarrow W W \rightarrow \ell \nu \ell \nu$ Channels in $360 \mathrm{pb}^{-1}$ CDF Run II Data, CDF internal note $7708(2005)$

[45] J. Conway, Efficiency Uncertainties: A Bayesian Prescription, CDF internal note 5894 (2002).

[46] T. Sjostrand and S. Mrenna, PYTHIA 6.2: Physics and Manual (2001).

[47] CTEQ Collaboration, CTEQ Parton Distributions and Flavor Dependence of Sea Quarks, Physisc Letter B 304159 (1993);

CTEQ Collaboration, Global QCD Analysis of Parton Structure of the Nucleon: CTEQ5 Parton Distributions, European Physical Journal C 12375 (2000).

[48] CDF Collaboration, PYTHIA Tune A, HERWIG and JIMMY in Run 2 at CDF, hep-ph/0510198 (2005). 
[49] R. Brun, R. Hagelberg, M. Hansroul and J. C. Lassalle, GEANT: Simulation Program for Particle Physics Experiments - User Guide and Reference Manual, CERN-DD-78-2(-REV) (1978); GEANT3, CERN-DD/EE/84-1 (1987).

[50] A. Taffard, TopNtuple: A Public Analysis Module for the Top Group, CDF internal note 6737 (2003) and 7267 (2004).

[51] W. K. Tung et al., Uncertainties of Predictions from Parton Distribution Functions: 2) the Hessian Method, Physical Review D 65014013 (2002).

[52] CDF Collaboration, First Measurements of Inclusive $W$ and $Z$ Cross Sections from Run II of the Tevatron Collider. Physical Review Letters 94091803 (2005).

[53] CDF Collaboration, Measurement of the $W^{+} W^{-}$Production Cross Section in $p \bar{p}$ Collisions at $\sqrt{s}=1.96 \mathrm{TeV}$ Using Dilepton Events, Physical Review Letters 94 $211801(2005)$

[54] CDF Collaboration, Search for $Z Z$ and $Z W$ Production in $p \bar{p}$ Collisions at $\sqrt{s}=$ 1.96 TeV, Physical Review D 71091105 (2005).

[55] M. Cacciari et al., the $t \bar{t}$ Cross-Section at $1.8 \mathrm{TeV}$ and $1.96 \mathrm{TeV}$ : A Study of the Systematics Due to Parton Densities and Scale Dependence, Journal of High Energy Physics 0404068 (2004).

[56] R. Hamberg, W. L. van Neerven, T. Matsuura, a Complete Calculation of the Order $\alpha_{s}{ }^{2}$ Correction to the Drell-Yan K Factor, Nuclear Physics B 359343 (1991). 
[57] U. Baur and E. L. Berger, Probing the $W W \gamma$ Vertex at the Fermilab Tevatron Collider, Physical Review D 411476 (1990).

[58] CDF Collaboration, Measurement of $W \gamma$ and $Z \gamma$ Production in $p \bar{p}$ Collisions at $\sqrt{s}=1.96 \mathrm{TeV}$, Physical Review Letters 94041803 (2005).

[59] T. Bayes, an Essay towards Solving a Problem in the Doctrine of Chances, Philosophical Transactions of the Royal Society of London 53370 (1763).

[60] J. Heinrich for the CDF Statistics Committee, Limit Calculations in the Presence of Nuisance Parameters: 1. Bayesian Approach, CDF internal note 7117 (2004).

[61] L. Demortier, A Fully Bayesian Computation of Upper Limits for Poisson Processes, CDF internal note 5928 (1st edition in 2002).

[62] R. D. Cousins, Why Isn't Every Physicist a Bayesian?, American Journal of Physics 635 (1995).

[63] J. Neyman, Outline of a Theory of Statistical Estimation Based on the Classical Theory of Probability, Philosophical Transactions of the Royal Society of London A 236333 (1937).

[64] S. Poisson, Recherches sur la Probabilité des Jugements en Matières Criminelles et Matière Civile (1838).

[65] R. Brun and F. Rademakers, ROOT: An Object Oriented Data Analysis Framework, Nuclear Instruments and Methods A 38981 (1997).

[66] CDF Collaboration, Search for a Neutral Higgs Boson Decaying to a $W$ Boson Pair in $p \bar{p}$ Collisions at $\sqrt{s}=1.96 \mathrm{TeV}$, Physical Review Letters 97081802 (2006). 